\title{
Studies in High Current Density Ion Sources for Heavy Ion Fusion Applications
}

\author{
by \\ Edwin Chacón-Gólcher
}

License (Universidad de Costa Rica) 1996

A dissertation submitted in partial satisfaction of the requirements for the degree of

Doctor of Philosophy

in

$$
\begin{gathered}
\text { Engineering - Nuclear Engineering } \\
\text { in the }
\end{gathered}
$$

GRADUATE DIVISION

of the

UNIVERSITY of CALIFORNIA at BERKELEY

Committee in charge:

Professor Edward C. Morse

Professor Per Peterson

Professor Wulf Kunkel

Fall 2002 
The dissertation of Edwin Chacón-Gólcher is approved:

Chair Date

Date

Date

University of California at Berkeley

Fall 2002 
Studies in High Current Density Ion Sources for Heavy Ion Fusion Applications

Copyright Fall 2002

By

Edwin Chacón-Gólcher 


\title{
Abstract \\ Studies in High Current Density Ion Sources for Heavy Ion Fusion Applications
}

\author{
By \\ Edwin Chacón-Gólcher \\ Doctor of Philosophy in Engineering - Nuclear Engineering \\ University of California at Berkeley \\ Professor Edward C. Morse, Chair
}

This dissertation develops diverse research on small (diameter $\sim$ few $\mathrm{mm}$ ), high current density $\left(\mathrm{J} \sim\right.$ several tens of $\left.\mathrm{mA} / \mathrm{cm}^{2}\right)$ heavy ion sources. The research has been developed in the context of a programmatic interest within the Heavy Ion Fusion (HIF) Program to explore alternative architectures in the beam injection systems that use the merging of small, bright beams. An ion gun was designed and built for these experiments. Results of average current density yield $(<\mathrm{J}>$ ) at different operating conditions are presented for $\mathrm{K}^{+}$and $\mathrm{Cs}^{+}$contact ionization sources and potassium aluminum silicate sources. Maximum $<\mathrm{J}>$ values for a $\mathrm{K}^{+}$beam of $\sim 90 \mathrm{~mA} / \mathrm{cm}^{2}$ were observed in $2.3 \mu$ s pulses. Measurements of beam intensity profiles and emittances are included. Measurements of neutral particle desorption are presented at different operating conditions which lead to a better understanding of the underlying atomic diffusion processes that determine the lifetime of the emitter. Estimates of diffusion times consistent with measurements are presented, as well as estimates of maximum repetition

\footnotetext{
${ }^{*}$ This work was performed under the auspices of the Office of Fusion Energy Science, U.S. Department of Energy by the University of California Lawrence Berkeley National Laboratory under contract No. DEAC03-765F00098.
} 
rates achievable. Diverse studies performed on the composition and preparation of alkali aluminosilicate ion sources are also presented. In addition, this work includes preliminary work carried out exploring the viability of an argon plasma ion source and a bismuth metal vapor vacuum arc (MEVVA) ion source. For the former ion source, fast rise-times $(\sim 1 \mu \mathrm{s})$, high current densities $\left(\sim 100 \mathrm{~mA} / \mathrm{cm}^{2}\right)$ and low operating pressures $(<$ 2 mtorr) were verified. For the latter, high but acceptable levels of beam emittance were measured $\left(\varepsilon_{\mathrm{n}} \leq 0.006 \pi \mathrm{mm} m \mathrm{mrad}\right)$ although measured currents differed from the desired ones (I $\sim 5 \mathrm{~mA})$ by about a factor of 10 .

Professor Edward C. Morse Dissertation Committee Chair 
To Virginia Gólcher, my mother.

Qué suerte para mi haberte encontrado, mami. 


\section{TABLE OF CONTENTS}

CHAPTER 1: INTRODUCTION

1.1 ON THE ORGANIZATION OF THIS DISSERTATION.

1.2 ON THE RATIONALE FOR MULTIPLE SMALL BEAMLET INJECTORS REQUIRING HIGH CURRENT DENSITY.

2.1 ON THE STRUCTURE AND NOMENCLATURE OF THE ALKALI ALUMINOSILICATES. 8

2.2 SCANNING ELECTRON MICROSCOPE STUDIES ON ALUMINOSILICATES. 16

2.2.1 PreParation of ALKali AluminosiLiCATES. 16

2.2.2 ELEMENT COMPOSITION DEPENDENCE ON PREPARATION TEMPERATURE. 21

CHAPTER 3: EXPERIMENTAL SETUP

3.1 EXPERIMENTAL TEST STAND 29

3.2 Mechanical Design of the Ion GuN 31

$\begin{array}{lll}3.3 & \text { Diagnostics } & 35\end{array}$

3.3.1 FARADAY CUP 35

3.3.2 Neutral Particle Detector 37

3.3.3 DOUBLE SLIT SCANNER. 41

CHAPTER 4: EXPERIMENTAL RESULTS $\quad 47$

4.1 CONTACt IONIZATION SOURCE PreParation. 48

$\begin{array}{ll}\text { 4.1.1 Doping Calculation. } & 48\end{array}$

4.2 High CURRent Density Yields OF $\mathrm{K}^{+} \mathrm{AND} \mathrm{CS}^{+}$Sources 49

4.2.1 Performance of High Current Density Potassium on Tungsten CONTACT IONIZATION SOURCES. 


\subsubsection{Performance of High Current Density Cesium on Tungsten}

CONTACT IONIZATION SOURCES.

4.2.3 Performance of High Current Density Potassium Aluminosilicate SOURCES.

4.2.4 EVIDENCE OF RECOVERY MECHANISMS IN A DEPLETED ALUMINOSILICATE SOURCE.

4.2.5 COMPosite MATERIAl Performance

4.3 NeUtral EMISSION MEASUREMENTS.

\subsubsection{NeUtral Particle Detector Design}

4.4 INTEGRATED PROFILE AND EMITTANCE MEASUREMENTS OF POTASSIUM DOPED AND ALUMINOSILICATE SOURCES.

CHAPTER 5: THEORETICAL CONSIDERATIONS.

5.1 The THEORY OF CONTACT IONIZATION SOURCES

5.1.1 SURFACE IONIZATION.

5.1.2 BRIEF REVIEW OF LANGMUIR'S CLASSICAL THEORY.

5.1.3 BASIC EQUATION FOR THE DESORPTION OF CS FROM A TUNGSTEN SURFACE.

5.2 ON THE DESORPTION PROBABILITY OF A PARTICLE FROM A SURFACE AND THE DROOPING OF CURRENT PULSES DURING THE EXTRACTION PULSE.

5.3 ON THE LIFETIME OF A CONTACT IONIZATION SOURCE.

5.3.1 ON THE DIFFUSION OF THE ALKALI SPECIES TOWARDS THE EMITTER SURFACE.

5.3.2 ON THE SURFACE REPLENISHMENT RATE.

5.3.3 ON THE DIFFERENCES OF OPERATION BETWEEN A PROPULSION ION THRUSTER AND THE STUDIED CONTACT IONIZATION SOURCES.

5.3.4 THEORETICAL REMARKS ON THE DIFFUSION IN POROUS MEDIA.

5.4 ESTIMATES OF APPARENT NEUTRAL PARTICLE COVERAGE. 
5.5 ON THE PRESENCE OF LARGE AMOUNTS OF IONIZABLE IMPURITIES AND THEIR EFFECT ON THE SPACE CHARGE LIMIT.

5.6 ON THE EFFECTS OF RESIDUAL ATMOSPHERES OF ALKALI GASES ON THE ION SOURCE CURRENT OUTPUT.

5.7 ION Gun Simulations AND SENSITIVITY ANALYSES.

5.7.1 MODELING OF THE GAP GEOMETRY.

6.1 Potassium Aluminum Silicate EMitTers.

6.2 CONTACT IONIZATION SOURCES.

6.3 GENERAL ISSUES.

APPENDIX 1: INITIAL EXPERIMENTS OF RF GAS PLASMA SOURCE FOR HEAVY ION FUSION *

APPENDIX 2:TIME RESOLVED EMITTANCE OF A BISMUTH ION BEAM FROM A PULSED VACUUM ARC ION SOURCE 


\section{LIST OF FIGURES}

Figure 1.1 Comparison of the sizes of a large emitter, low $\mathrm{J}$ design with a multiple high $\mathrm{J}$ beamlet design.

Figure 1.2 Detail of the dimensions of a multiple-beamlet injector.

Figure 1.3. Aperture configuration at the last accelerating plate (left) and $\mathrm{X}$ and $\mathrm{Y}$ beam radii on merge region and entrance to the transport lattice.

Figure 2.1. $\mathrm{SiO}_{4}$ group, elementary building block of the silicates.

Figure 2.2. Clasification of the silicates by structure.

Figure 2.3. Phase diagram of the $\mathrm{K}_{2} \mathrm{O}-\mathrm{Al}_{2} \mathrm{O}_{3}-\mathrm{SiO}_{2}$ system.

Figure 2.4 Structure of the mineral leucite in its low temperature form. Color Code: Green: K, Red: O, Al and Si occupy the space inside the purple tetrahedrons. 15

Figure 2.5. Alternative view of the leucite structure where the apertures in the structure can be appreciated. Same color coding as in Figure 2.5.

Figure 2.6. Scanning Electron Microscope X - ray signature of a potassium aluminosilicate powder.

Figure 2.7. Intensity map on an element basis. The width of each of these maps represents a length of $\sim 24 \mu \mathrm{m}$ in the original sample.

Figure 2.8. Potassium aluminosilicate spectrum depicting a zirconium impurity. 20

Figure 2.9. Element distribution map corresponding to Fig. 2.8. Note again positive correlation among the elements. Higher intensity spots on the $\mathrm{Al}$ seem to be aluminum oxide specs on the surface. Horizontal width of each map corresponds to a length of $\sim 120 \mu \mathrm{m}$ in the original surface.

Figure 2.10. Spectrum of an aluminosilicate source prepared at $1500^{\circ} \mathrm{C}$ in an argon atmosphere. Glassy appearance with specs of aluminum oxide. 22

Figure 2.11. Element distribution corresponding to the previous figure. Horizontal length in each map corresponds to $\sim 30 \mu \mathrm{m}$ in the original surface. Note again high positive correlation among the elements except for the aluminum oxide specs. 22

Figure 2.12. Spectrum of an aluminosilicate source prepared at $1550^{\circ} \mathrm{C}$ in an argon atmosphere. 23 
Figure 2.13. Photograph of the aluminosilicate prepared at $1,550^{\circ} \mathrm{C}$. Note the aluminum oxide specs on the surface. 1,000X magnification.

Figure 2.14. Element distribution of the aluminosilicate prepared at $1,550^{\circ} \mathrm{C}$. Image corresponds to the very central part of the previous photograph. 24

Figure 2.15. Spectrum of an aluminosilicate source prepared at $1,610^{\circ} \mathrm{C}$ in vacuum. Note the significant decrease of potassium. 25

Figure 2.16. Element distribution map of the aluminosilicate prepared at $1,610^{\circ} \mathrm{C}$. There is evidence of some degree of separation between $\mathrm{Al}$ and $\mathrm{Si}$ at this level. 26

Figure 2.17. Spectrum of an aluminosilicate source prepared at $1,660^{\circ} \mathrm{C}$ in an argon atmosphere. Note the significant decrease of potassium.

Figure 2.18. Element distribution map of the aluminosilicate prepared at $1,660^{\circ} \mathrm{C}$. At this temperature, there is a significant separation of the components. Silicon and aluminum are now anti-correlated. The little potassium that remains is associated with the $\mathrm{Si}$ while the dendrites appear to be formed out of aluminum oxide. 27

Figure 3.1 Setup Photograph 30

Figure 3.2. Main heater cage showing filaments and main source emitter. 32

Figure 3.3. Heater module. 32

Figure 3.4. Typical dependence of the source temperature with respect to the electrical power input. 33

Figure 3.5. High J ion gun with extraction plate on. 34

Figure 3.6. 3-D CAD half-view representation of the used Faraday Cup. 35

Figure 3.7. Faraday Cup dimensions, in inches. 36

Figure 3.8 Coupling circuit diagram. 36

Figure 3.9 Schematic of the neutral particle detector. 38

Figure 3.10. Dependence of the shape factor between the $1 / 4$ " source and the Pt foil in the neutral detector on the distance between them. 40

Figure 3.11. CAD representation of the neutral detector. 40

Figure 3.12 Schematic of the action of the double slit system for measuring the emittance. (Taken from MacLaren, 2000.) 42 
Figure 4.1 Maximum current density output performance observed for a potassium doped contact ionization source.

Figure 4.2 Scope trace showing the high voltage (positive pulse) and the current pulse . 51

Figure 4.3 Decreased current output of a potassium doped tungsten source after 17 hours of continual operation at $1.145^{\circ} \mathrm{C}$. 52

Figure 4.4. $6 \mu \mathrm{m}$ grain nominal size, sintered tungsten emitter, near center spot. Firing conditions: $1,950 \mathrm{C}, 3 \mathrm{~h}$ in vacuum. 53

Figure 4.5. Same emitter as in Figure 4.46, different region near the center spot displaying one of the multiple regions of no porosity. 53

Figure 4.6. Same emitter substrate as shown in Figure 4.4. Region near the edge of the emitter. Note the differences in porosity with resptect to the center of the emitter. 54

Figure 4.7. $\mathrm{J}-\mathrm{V}$ characteristics of a potassium on porous tungsten contact ionization source. 55

Figure $4.8 \mathrm{~J}-\mathrm{V}$ characteristics of a potassium doped tungsten source after almost $17 \mathrm{hr}$. of operation at $1,075^{\circ} \mathrm{C}$. 57

Figure 4.9. $\mathrm{J}-\mathrm{V}$ characteristics of a potassium doped tungsten source after $50 \mathrm{hr}$. of operation. Last 33 hours at $1,100{ }^{\circ} \mathrm{C}$. 58

Figure 4.10. Rapid decrease of output current in a potassium doped tungsten source at $1,300{ }^{\circ} \mathrm{C}$. 59

Figure 4.11. $\mathrm{J}-\mathrm{V}$ characteristics of a recently doped cesium on tungsten contact ionization source. 61

Figure 4.12. Current output at a constant extraction voltage of a Cs on $\mathrm{W}$ source at $\mathrm{T}=$ $1,090{ }^{\circ} \mathrm{C}$. 62

Figure 4.13. $\mathrm{J}-\mathrm{V}$ characteristics of a cesium doped tungsten source after $18 \mathrm{hr}$. of operation at $1,100{ }^{\circ} \mathrm{C}$ and reactivation after the ion output previously decayed to zero. 63

Figure 4.14. $\mathrm{J}-\mathrm{V}$ characteristics of a cesium doped tungsten source after almost $50 \mathrm{hr}$. of operation at $1,100^{\circ} \mathrm{C}$. 64

Figure 4.15. Average current output of a Cs source at a constant extraction voltage and temperature of $1,060^{\circ} \mathrm{C}$. 65 
Figure 4.16. Average current output of a Cs source at a constant extraction voltage and temperature of $1,120^{\circ} \mathrm{C}$. 66

Figure 4.17. Hysteresis effect caused by the influence of the ion desorption on the surface coverage. Operating temperature $=1,120^{\circ} \mathrm{C}$.

Figure 4.18. Family of $\mathrm{J}-\mathrm{V}$ characteristics measured at different points of the lifetime test presented in Figure 4.16. 68

Figure 4.19 $\mathrm{J}-\mathrm{V}$ characteristic of a re-doped Cs on $\mathrm{W}$ source after 2 days of pre-heating at $985^{\circ} \mathrm{C}$.

Figure 4.20 Output vs. time at a constant voltage of a re-doped Cs on tungsten emitter, displaying an unexpected decrease of output with time. 70

Figure 4.21 Abrupt activation of a $\mathrm{Cs}$ on $\mathrm{W}$ source after its operating temperature went beyond $1,100{ }^{\circ} \mathrm{C}$. 71

Figure 4.22 $\mathrm{J}-\mathrm{V}$ performance of the activated $\mathrm{Cs}$ on $\mathrm{W}$ doped source at an operating temperature of $1,150^{\circ} \mathrm{C}$. 72

Figure 4.23 Cooling down cycle of a Cs on tungsten source showing the maximum average current densities achievable at the different temperatures in an emission limited mode. 72

Figure 4.24 Comparison of the maximum average current density measured for the Cs doped source compared with the maximum expected theoretical current density. 73

Figure 4.25 Average current density variation with time during the heating-up cycle of a potassium aluminosilicate source, at different operating temperatures and fixed extraction voltage. 75

Figure 4.26. Continuation of the life test of figure 4.27 showing increased current output with time at a fixed temperature, and the discontinuities that occur after the emitter has been subject to peak extraction voltages during $\mathrm{J}-\mathrm{V}$ characterization. 76

Figure 4.27 Performance characteristics of a potassium aluminosilicate source. Curves (b), (c), (d), (e) and (f) were measured after 24, 28, 48, 78 and $119 \mathrm{hr}$ of operation at $1,100{ }^{\circ} \mathrm{C}$ respectively. 78

Figure 4.28. Comparison between a recently manufactured aluminosilicate pellet (gray) and a fully activated emitter after $\sim 220 \mathrm{hrs}$. of operation.

Figure 4.29. Change in the position of an aluminosilicate source within the Pierce electrode after almost 220 hours of operation. 80 
Figure 4.30. Performance characteristics of a potassium aluminosilicate ion source. Curves named (b), (c), (d), (e) and (f) were measured at 24, 48, 72, 120 and 145 hours of operation at $1,100{ }^{\circ} \mathrm{C}$ respectively.

Figure 4.31 Long term time dependence of the average current density output of a potassium alumilnosilicate source at a fixed extraction voltage. 82

Figure 4.32 $\mathrm{J}-\mathrm{V}$ characteristics of a potassium aluminosilicate emitter showing a vertical translation of the space charge limit line. Note the distinct parallel location of the points measured in the days 8 and 9 of the test in contrast with the data sets taken later. 83

Figure $4.33 \mathrm{~J}-\mathrm{V}$ charateristics of a fully activated potassium aluminosilicate source. Contrast these curves with that of the non-activated source presented in Figure 4.30. 84

Figure 4.34 Close-up view of the original position of the K-aluminosilicate emitter in the Pierce electrode. 85

Figure 4.35. Close-up view of the K-aluminosilicate source after almost 15 days of continual operation. 86

Figure 4.36. Initial damage of the K-aluminosilicate emitter upon contact with the knifeedge of the Pierce electrode. 87

Figure 4.37 Complete separation of the K-aluminosilicate emitter from the tungsten substrate upon the application of a slight pressure. 88

Figure 4.38. SEM photograph of the surface of the tungsten substrate, showing a surface with no porosity. The scratches are presumably the effect of a sliding between the aluminosilicate layer and the tungsten surface. 89

Figure 4.39. SEM photograph of a different region of the original aluminosilicatetungsten interface, showing the tungsten grain growth and subsequent pore closing. Note the crystals of aluminum oxide that have remained attached to the tungsten. 90

Figure 4.40 View of the back side of the aluminosilicate that separated from the tungsten substrate. The protrusions that can be observed may have been points of original contact or penetration into the tungsten substrate, before the latter began to close. The $2 \mathrm{kX}$ magnification yields a $5 \mu \mathrm{m}$ length of the reference line. 91

Figure 4.41 SEM photograph of the original appearance of the tested aluminosilicate emitter. The aluminosilicate is the glass-like smooth surface. 92

Figure 4.42 View of the front side of the aluminosilicate that separated from its substrate. 93

Figure 4.43 SEM spectrum of the front side of the aluminosilicate. 93 
Figure 4.44 Spectrum of the back side of the aluminosilicate part.

Figure 4.45. Partially destructed aluminosilicate layer due to the action of backstreaming electrons in a 14 minute DC test. 97

Figure 4.46 Summary of the DC forced extraction test for depletion of an aluminosilicate source. Note: total current has an electron component and must be adjusted to estimate the total number of ions delivered. 98

Figure 4.47. Second activation of a $\mathrm{K}$ aluminosilicate emitter. A problem in the data acquisition system provoked a loss of a fraction of the data. Nevertheless, the information is sufficiently revealing as to the source performance. Contrast with Figure 4.31 , the activation occurs in a similar period of time but lower temperatures are required.

Figure 4.48 Recovery of an aluminosilicate source after a forced depletion with a DC voltage. The current values have been averaged over the original area of the emitter, but it must be reminded that the actual emitting area was only a fraction of the original._ 100

Figure 4.49 Comparison of $\mathrm{J}-\mathrm{V}$ characteristics before and after depletion of the aluminosilicate emitter. The "depleted" curve was generated within $1 \mathrm{hr}$ of the depletion.

101

Figure 4.50. Ratio of the current value of the reactivated emitter after depletion to the original value of the current previous to the depletion. 103

Figure 4.51. X-Ray spectrum of the depleted aluminosilicate pellet after re-activation. 103

Figure 4.52. Performance at different operating temperatures of a Mo-K-aluminosilicate composite. 105

Figure 4.53. Backscattered electron photograph of the surface of a molybdenum-K aluminosilicate composite pellet near its center. Brighter grains correspond to Mo. Note the wide distribution of Mo grain sizes around the nominal size of $6 \mu \mathrm{m}$. 106

Figure 4.54. Backscattered electron photograph of the surface of a molybdenum-K aluminosilicate composite pellet, near the edge. Note the increased Mo content. 106

Figure 4.55 Life test of a potassium aluminosilicate/tungsten composite pellet. 107

Figure 4.56 $\mathrm{J}-\mathrm{V}$ characteristic of a tungsten/K aluminosilicate composite, operating at a temperature of $1,000^{\circ} \mathrm{C}$. 108

Figure 4.57. Sample data set of a heating cycle of Cs and K contact ionization sources measured with the neutral detector. 111 
Figure 4.58. Sample data representing the signal at the neutral detector as a function of position relative to the emitter. Data such as in Figure 4.58 were measured at the central position, directly in front of the ion source.

Figure 4.59. Data for a rough estimation of the desorption energies of Cs and $\mathrm{K}$ neutral particles. 115

Figure 4.60. Ratio of the measured (pulsed) ion yield at different temperatures to the corresponding (DC) neutral particle emission rate. 116

Figure 4.61. Slit-cup signal as a function of position and time of a potassium doped source operating at $1,045^{\circ} \mathrm{C} . \mathrm{I}=1.24 \mathrm{~mA} . \quad\left\langle\mathrm{J}>=4.4 \mathrm{~mA} / \mathrm{cm}^{2}\right.$. 117

Figure 4.62. Beam profile at mid pulse of the potassium beam. The asymmetry of the beam is partially due to an increase of the beam current with time. $\mathrm{T}=1,045^{\circ} \mathrm{C}$. 119

Figure 4.63. Time-resolved beam profile of the potassium beam. $\mathrm{T}=1,075^{\circ} \mathrm{C} . \mathrm{I}=4$ $\mathrm{mA} .<\mathrm{J}>=14.18 \mathrm{~mA} / \mathrm{cm}^{2}$. 119

Figure 4.64. Beam profile at mid pulse of the potassium beam. $\mathrm{T}=1,075^{\circ} \mathrm{C}$. 120

Figure 4.65 Phase space plot of the potassium beam. Source temperature: $1,075{ }^{\circ} \mathrm{C}$. Normalized emittance: $6.14 \cdot 10^{-2} \pi \mathrm{mm}$ mrad. 121

Figure 4.66. Same phase space as in Figure 4.64 but sheared to appreciate structure. 121

Figure 4.67. Time-resolved beam profile of the potassium beam. $\mathrm{T}=1,075{ }^{\circ} \mathrm{C} . \quad \mathrm{I}=8.6$ $\mathrm{mA} .<\mathrm{J}>=30.5 \mathrm{~mA} / \mathrm{cm}^{2}$. Current is higher due to better surface coverage. 122

Figure 4.68. Beam profile at mid pulse of the potassium beam after improvement of the surface coverage conditions. $\mathrm{T}=1,075^{\circ} \mathrm{C}$. 123

Figure 4.69. Time-resolved beam profile of the potassium beam. $\mathrm{T}=1,150{ }^{\circ} \mathrm{C} . \mathrm{I}=11.9$ $\mathrm{mA} .<\mathrm{J}>=42.2 \mathrm{~mA} / \mathrm{cm}^{2}$. 123

Figure 4.70. Beam profile at mid pulse of the potassium beam. $\mathrm{T}=1,150{ }^{\circ} \mathrm{C}$. 124

Figure 4.71 Phase space plot of the potassium beam. Source temperature: $1,150{ }^{\circ} \mathrm{C}$. Normalized emittance: $2.69 \cdot 10^{-2} \pi \mathrm{mm}$ mrad. 125

Figure 4.72. Time-resolved beam profile of the potassium beam. $\quad \mathrm{T}=1,205^{\circ} \mathrm{C} . \quad \mathrm{I}=$ $12.44 \mathrm{~mA} .<\mathrm{J}>=44.1 \mathrm{~mA} / \mathrm{cm}^{2}$. 125

Figure 4.73 Phase space plot of the potassium beam. Source temperature: $1,205{ }^{\circ} \mathrm{C}$. Normalized emittance: $3.44 \cdot 10^{-2} \pi \mathrm{mm} m$ mad. 126 
Figure 4.74. Time-resolved beam profile showing the characteristic depletion features.

Figure 4.75. Oscilloscope traces showing the short $(\sim 2 \mu \mathrm{s})$ high voltage pulse (upper trace) and the faraday cup signal (lower trace) of a depleting ion source.

Figure 4.76. Family of mid-pulse profiles at a fixed voltage of a potassium aluminosilicate type source, at different temperatures of operation. 128

Figure 4.77 Current emission from a potassium aluminosilicate source as a function of surface temperature. 129

Figure 4.78 Current emission from a potassium aluminosilicate source as a function of surface temperature. 130

Figure 4.79. Emittance plot of a potassium aluminosilicate source operating at 945 C.131

Figure 4.80 Time resolved profile of a potassium aluminosilicate source. $\mathrm{T}=1,035{ }^{\circ} \mathrm{C}$. 131

Figure 4.81 Time resolved profile of a potassium aluminosilicate source. $\mathrm{T}=1,100{ }^{\circ} \mathrm{C}$. 132

Figure 4.82 Phase space measurement of a potassium aluminosilicate source. $T=1,075$ ${ }^{\circ} \mathrm{C}$.

Figure 4.83 Phase space measurement of a potassium aluminosilicate source. $T=1,050$ ${ }^{\circ} \mathrm{C}$.

Figure 5.1. Atom $\left(v_{\mathrm{a}}\right)$ and ion $\left(v_{\mathrm{p}}\right)$ desorption rates for Cs on a tungsten surface as a function of surface coverage and temperature.

Figure 5.2 Diagram representing the nomenclature for the energetics of ion, atom and electron desorption from a surface, in the case of Cs. 142

Figure 5.3 Life test of a cesium on tungsten source, at an operating temperature of 1,125 ${ }^{\circ} \mathrm{C}$. Current density measureents were made at the center of the pulse. 145

Figure $5.4 \mathrm{~J}-\mathrm{V}$ characteristics of the ion source corresponding to different points in time of the test of Figure 5.2. 146

Figure 5.5 Comparison of the $\mathrm{J}-\mathrm{V}$ characteristics of the depleting source when measuring at the beginning versus the middle of the pulse. 147

Figure 5.6. Flat reference pulse of the Cs beam mentioned above, during normal operation. Upper trace is the High Voltage pulse. 148 
Figure 5.7. Current pulse shape at an extraction voltage of $19.5 \mathrm{kV}$, after 160 hrs. of operation at $1,125^{\circ} \mathrm{C}$. 148

Figure 5.8. Current pulse shape at an extraction voltage of $23.5 \mathrm{kV}$, after $160 \mathrm{hrs}$. of operation at $1,125^{\circ} \mathrm{C}$.

Figure 5.9. Current pulse shape at an extraction voltage of $27.3 \mathrm{kV}$, after $160 \mathrm{hrs}$. of operation at $1,125^{\circ} \mathrm{C}$. 149

Figure 5.10. Current pulse shape at an extraction voltage of $33.1 \mathrm{kV}$, after $160 \mathrm{hrs}$. of operation at $1,125^{\circ} \mathrm{C}$. 150

Figure 5.11. Current pulse shape at an extraction voltage of $37.4 \mathrm{kV}$, after $160 \mathrm{hrs}$. of operation at $1,125^{\circ} \mathrm{C}$. 150

Figure 5.12. Expected time evolution of the current density level of a cesium on tungsten doped emitter, operating in the emission limited mode, with the initial Cs surface coverage level used as a parameter. Source temperature: $1,125^{\circ} \mathrm{C}$. 154

Figure 5.13. Time evolution of the delivered current density by a cesium contact ionization source, showing the effect on the delivered current due to the changes in the surface alkali content provoked by the current pulse. Temperature $=1,050^{\circ} \mathrm{C}$. 156

Figure 5.14. Time evolution of the delivered current density by a cesium contact ionization source, showing the effect on the delivered current due to the changes in the surface alkali content provoked by the current pulse. Temperature $=1,100^{\circ} \mathrm{C}$. 156

Figure 5.15. Time evolution of the delivered current density by a cesium contact ionization source, showing the effect on the delivered current due to the changes in the surface alkali content provoked by the current pulse. Temperature $=1,150^{\circ} \mathrm{C}$. 157

Figure 5.16. Time evolution of the delivered current density by a cesium contact ionization source, showing the effect on the delivered current due to the changes in the surface alkali content provoked by the current pulse. Temperature $=1,200^{\circ} \mathrm{C}$. 157

Figure 5.17. Time evolution of the delivered current density by a cesium contact ionization source, showing the effect on the delivered current due to the changes in the surface alkali content provoked by the current pulse. Temperature $=1,250^{\circ} \mathrm{C}$. 158

Figure 5.18. Time evolution of the delivered current density by a cesium contact ionization source, showing the effect on the delivered current due to the changes in the surface alkali content provoked by the current pulse. Temperature $=1,300^{\circ} \mathrm{C}$. 158

Figure 5.19. Schematic of the diffusion problem solved to model the migration of the alkali atoms through the porous tungsten substrate. 163 
Figure 5.20. Fitting of the Cs neutral desorption data at a temperature of $680{ }^{\circ} \mathrm{C}$. Diffusion constant: $5.9 \cdot 10^{-6} \mathrm{~cm}^{2} / \mathrm{s}$ 168

Figure 5.21. Fitting of the Cs neutral desorption data at a temperature of $765{ }^{\circ} \mathrm{C}$ with equation 5.15. Diffusion constant: $1.2 \cdot 10^{-5} \mathrm{~cm}^{2} / \mathrm{s}$ 168

Figure 5.22. Fitting of the Cs neutral desorption data at a temperature of $800{ }^{\circ} \mathrm{C}$. Diffusion constant: $3.7 \cdot 10^{-6} \mathrm{~cm}^{2} / \mathrm{s}$ 169

Figure 5.23. Fitting of the Cs neutral desorption data at a temperature of $840{ }^{\circ} \mathrm{C}$. Diffusion constant: $1.0 \cdot 10^{-5} \mathrm{~cm}^{2} / \mathrm{s}$. 170

Figure 5.24. Fitting of the Cs neutral desorption data at a temperature of $1,025{ }^{\circ} \mathrm{C}$. Diffusion constant: $1 \cdot 10^{-6} \mathrm{~cm}^{2} / \mathrm{s}$ 171

Figure 5.25. Fitting of the Cs neutral desorption data at a temperature of $1,110{ }^{\circ} \mathrm{C}$. Diffusion constant: $7.5 \cdot 10^{-5} \mathrm{~cm}^{2} / \mathrm{s}$ 171

Figure 5.26. Fitting of the Cs neutral desorption data at a temperature of $1,130{ }^{\circ} \mathrm{C}$. Diffusion constant: $5.7 \cdot 10^{-6} \mathrm{~cm}^{2} / \mathrm{s}$ 172

Figure 5.27 J-V characteristics of the cesium on tungsten ion source used for testing of the neutral evaporation rates and diffusion constants. 174

Figure $5.28 \mathrm{~J}-\mathrm{V}$ characteristics of the potassium on tungsten ion source used for testing of the neutral evaporation rates and diffusion constants. 174

Figure 5.29. Dependence of the maximum repetition rate available in a contact ionization source as a function of the bulk diffusion constant, as measured in a $12 \mu \mathrm{m}$ nominal grain size emitter. The curves are parametrized by the grain size at the surface of the emitter if it can be made of a different size as the bulk material. The diffusion length is then taken as half of the nominal grain size at the surface. 179

Figure 5.30. Maximum repetition rate estimated with the same methodology as in Figure 5.29. The ratio of the diffusion rate versus the desorption rate was given a higher value, thus making the diffusion constant estimates less conservative. Diffusion constant values differ by a factor of $\sim 1.67$ with respect to the previous figure. 180

Figure 5.31. Apparent neutral concentration of cesium atoms on the porous tungsten surface (12 $\mu \mathrm{m}$ nominal grain size), as estimated from the neutral particle desorption rate data at the beginning of the time period at each temperature. Time periods were not of the same length. 191

Figure 5.32. Solution region for the modeling of the beam extraction with Trak 5.0. 201 
Figure 5.33. Trak 5.0 modeling of the Pierce - emitter gap influence on the initial extraction optics.

Figure 5.34. Plot of the expected $\mathrm{J}-\mathrm{V}$ characteristics and their dependence on the Pierce - emitter gap. 203

Figure 5.35. Track 5.0 modeling of the effect of the Pierce - emitter gap on the current density distribution as a function of emitter radius. 


\section{LIST OF TABLES}

Table 4.1. Summary of emittance measurements including effective ion temperature and normalized brightness.

Table 5.1. Summary of time and energy desorption parameters for cesium ions on tungsten (From Scheer and Fine, 1962 and Langmuir, 1933).

Table 5.2. Comparison of measured droop in a depleted Cs source with the predicted droop based on a simple model. The model gives more realistic results at higher operation currents.

Table 5.3. Values for the first four terms of the series of equation (5.13). 165

Table 5.4. Estimated diffusion constants of the alkali species through a nominal12 $\mu \mathrm{m}$ grain size porous tungsten substrate. Calculated by fitting the measured neutral desorption curves with equation (5.16). Several values for $\mathrm{D}$ are listed for the temperature of $955{ }^{\circ} \mathrm{C}$ as it is at this temperature that the source is left by itself to eliminate the surplus of alkali atoms and during a period that lasts $\sim 17$ hours. Lower diffusion constants are estimated at longer times. In general, the uncertainty of the values can be estimated to \pm 5 in the last decimal place to have curve fits of the type of agreement shown in Figures 5.20 through 5.26. 173

Table 5.5. Classification of diffusive fluxes based on the characteristic scale lengths. 186 


\section{Acknowledgements}

There is a story of a father that wanted to teach a lesson to his child. The father said: "Son, I want you to tell me if with all the strength you have, you can lift that log laying over there.” - "Let me try", his son replied. And then grabbed and end of the log with his hands and pulled it as hard as he could. The log failed to move even a little. -"I can't move it!," he said. After giving him a compassionate look, his father said "No son, you didn't do as I asked". Then he applied himself to the task of grabbing the log and lifting it with his own hands. "You didn't ask me for help, and all your strength, includes the strength of those who can support you."

I happened to remember this short story as an allegory of what happens in a multitude of occasions throughout one's life, and in the present context, to my academic experience during all these past 5 years at my beloved University of California at Berkeley. I can track all my humble accomplishments to the kindness of people that were always there, willing to give me a hand in one way or another. I start writing this section of acknowledgements with uttermost apprehension, not because of any reservation against thanking all the people that have helped me, a little or a lot during this time, but with fear of the certainty that many will be unintentionally left out. At the end of such a long process it is easy to forget how much one has received. Despite my efforts to do justice with my gratitude, I will always fall short given all I have received.

I will start by acknowledging my endless gratitude to the University of California, who through its Nuclear Engineering Department, and through processes, decisions, and 
people that will always be unknown to me, decided to grant me the opportunity to come here from a distant place, to re-invent my life.

In these same terms I will always be indebted to the Lawrence Berkeley National Laboratory, for all that this institution has done for me. In particular, my gratitude goes to the Heavy Ion Fusion Group, who welcomed me with enthusiasm and has provided the most wonderful environment to learn and to interact with people I utterly admire and respect. I want to thank Joe W. Kwan, my immediate supervisor, for his continual patience with my slow development and for letting me try to walk by myself, despite my frequent falls. Thank you Joe for your advice and guidance and for being easy on me even in moments you probably shouldn't have. I wish the final product of my work justifies in some way all that you and the HIF group have so generously given to me all these years.

I want to show my gratitude to my professor, Edward C. Morse, for the continual guidance and support that I have received from him since the first day I stepped clueless into Etcheverry Hall. Thank you for believing in me and taking me by the hand to help me find opportunities of every sort in and out of campus, for my academic and professional development.

I thank the members of my dissertation committee, Professors Wulf Kunkel and Per Peterson, for their always having an open door for me. Their corrections and insights in the problems that I attempted to solve added significantly to my humble efforts and understanding. Professor Kunkel, thank you for your continued presence and advice in our Plasma Physics Group Seminar, the history you brought has been an inspiration for all. 
I am forever indebted to my co-workers and friends William Ghiorso and Takeshi Katayanagi, for always being there to help me out and for teaching me so much. How much you have added to my existence and how much I really owe you is something I can't summarize in one line, in the same way I can't forget.

Throughout these years at LBNL I have experienced the hospitality and friendliness of the members of the HIF group. All of you have made my experience here so positive. I only regret not having had the time to interact more with such outstanding scientists that could have taught me so much more. My words of appreciation will never be enough to explain how great an environment you make and how much in one way or another you have personally added to my growth. Thanks Ed, for letting me see the power and depth of theory and for letting me come here for the first interview; Frank, for giving me an example of how to pursue new ideas; Larry for being such a patient scientific collaborator and for making the experience of working with you so much fun; Enrique, for always having time for advising and helping me; Simon and Shmuel, for being always so good and generous to me and letting me collaborate with you; John and Peter, for your continual kindness with me and willingness to help me out when I've needed it; Andy, Roger, Grant, Chris, Steve, Alex, Dave, Bill and Derek, for making this a great place to work, my interaction with you has been a continual inspiration to improve myself and to realize how lucky I have been to be here. Thank you Sharon, Lynn and Paijoun for your continued support in every way and your friendship during all this time. Thanks to my fellow student friends, for making all these experiences to be dealt with so more easily, thanks Michiel, for telling me about the HIF group; Steve, that's some 
legacy you left here man; Will, Dave, Lionel, Lance let's keep going and see where it all ends up!

I'm grateful to the very capable team in building 58. At some moment, almost everyone has played the role of instructor, be it in electronics or machining. To all of you guys, especially Ralph, Ron, Wayne, Jerry, Bill T., Bill S., Ed, Craig, I owe a lot.

To Dan Dietderich I owe his teachings in how to use the electron microscope and to give me access to it basically at any time I fancied. Thanks to James Wu for his perpetual support and interest in all the ion source preparation activities, you have been so important in this process. Thanks Dave Baca for all the support you have given me in ion source preparation activities.

I am thankful with my friends, all the wonderful relationships that I have built here throughout this time, all whom have taught me so much. Thanks Sassy for sharing so much with me and making me a better person. Thanks, Vlasta, for your friendship; life is a trip, isn't it? Thanks, Fina, Juergen, Dil, Sreby, Igor, Aida, Marie-Pierre, Xin, Tanja, Julia, for rewarding me with your friendship despite my quirks. How unfair to mention names here, with so many people that will always have a place in my heart. But one always has to try, at least with those who were visitors in Berkeley and in my life for a longer term than most.

To the people that I have left behind in my past and native country, thank you and I love you. Thanks Oscar Coto, my mentor in Costa Rica, your example and inspiration was key to get me to where I am now. Thanks Lorena, I celebrate more that we are friends than cousins. And to all my friends that live in my heart, time goes by and we change, but at any time we continue where we left off. 
I finally want to show my infinite gratitude to this country that has welcomed me with open arms, and to let me pursue my own American dream.

\section{Edwin Chacón Gólcher}

Berkeley, October 2002 


\section{Chapter 1: Introduction}

\subsection{On the organization of this dissertation.}

The words in the following pages summarize the efforts and experiments carried out in the Heavy Ion Fusion group at LBNL on the topic of high current density ion sources. The present chapter presents the introductory notes regarding the justification for this research, and the interest in the operation of contact ionization sources and aluminum silicate sources on these regimes of operation.

Chapter 2 deals with diverse studies carried out on the topic of potassium

aluminum silicate sources, more specifically, on the conditions most appropriate for yielding high current density capabilities on this type of material. The main diagnostic used in this section was a scanning electron microscope.

Chapter 3 presents the experimental apparatuses used in these experiments. All relevant features of design and construction are detailed in this chapter. 
Chapter 4 details the experimental results on a variety of ion sources tested. Potassium and Cesium contact ionization source results are presented, as well as the diverse findings on potassium aluminum silicate sources. Average current densities observed as a function of surface temperature, as well as current output as a function of time are presented. Similar results are presented for tests on composite sources fabricated with mixtures of aluminum silicate powders and molybdenum and tungsten powders. The chapter closes with double and single slit measurements of integrated beam profiles and emittance measurements. Much of the information presented in this chapter is presented in a practically anecdotal manner. To some extent this follows the "ion sourcery" tradition, in which the ion sources sometimes tend to behave in an almost capricious way, despite being prepared and operated in what would be thought as equivalent conditions. This is addressed and partially explained in the following chapter.

Chapter 5 is an attempt to provide a stronger conceptual foundation to many of the observations and measurements described in Chapter 4. Most important, is the attempt to estimate the diffusion properties of the alkali atoms within the porous tungsten substrate. These considerations have been made possible by the high sensitivity of the neutral detector used in these experiments. These estimates have made possible further exploration of questions such as the rates at which the surface is replenished, and from it, some rough guesses as to what are the repetition rates achievable by ion sources. These estimates are very preliminary but open the doors for more detailed analyses in this regard, making possible the full understanding and theoretical prediction of the ion source behavior in the case of contact ionization sources.

Chapter 6 is a summary of the most relevant conclusions delivered by this work. 
Two appendices present information on preliminary experiments carried out by the author and collaborators on the topics of a plasma ion source and a MEVVA type ion source, both candidates for use in the multiple beamlet architecture.

\subsection{On the rationale for multiple small beamlet injectors requiring high current density.}

The traditional concept for a future HIF driver injector involves the use of close to a hundred beams, each with a current on the order of $1 \mathrm{~A}$ and a normalized emittance of around $1 \pi$ mmmrad. Voltage breakdown and space charge limited emission considerations dictate constraints for the current yield and geometry of a single beam. The result is that the more current is required, the larger is the required size of the emitter and the lower is the current density. The use of multiple beamlets avoids this scaling. In the same way that in the traditional architectures the total current requirement can be supplied by several tens of $1 \mathrm{~A}$ beams, it is possible to use multiple beamlets to supply the required current per beam. A multiple beamlet injection system may provide significant advantages:

1. Smaller beam envelope excursions.

2. Increased control over the shaping and aiming of the beam.

3. Increased flexibility in the types of ion sources that can be used.

Figure 1.1 shows a representation of the potential advantages in volume reduction of the injector system with a multiple-beamlet design. The figure compares the outermost beamline in a multiple large emitter injector concept with the expected size of a multiple-small beamlet equivalent current beamline (Kwan, 2001). 


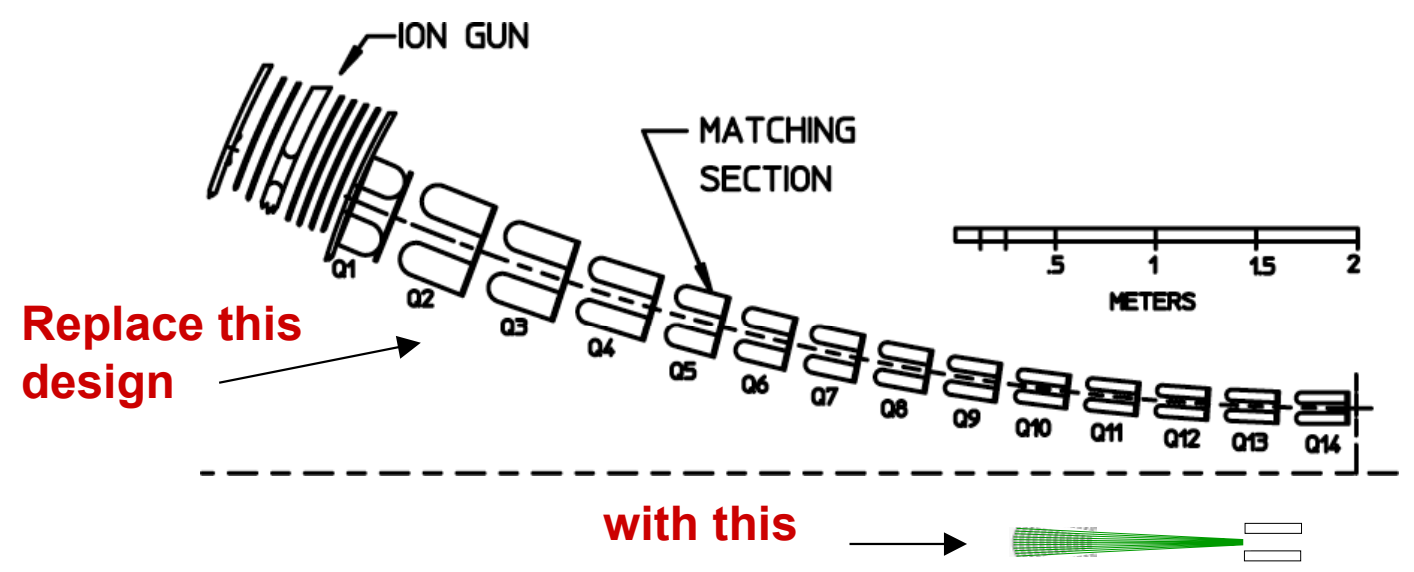

Figure 1.1 Comparison of the sizes of a large emitter, low J design with a multiple high $\mathrm{J}$ beamlet design.

The reduction in size is accomplished by matching the beam requirements at the start of a transport lattice through an adequate geometrical configuration of the aperture plates (Einzel lens transport system). The elliptical arrangement allows for exact matching into quadrupole transport channel. Figures 1.2 and 1.3 show a larger geometric detail of one such concept.

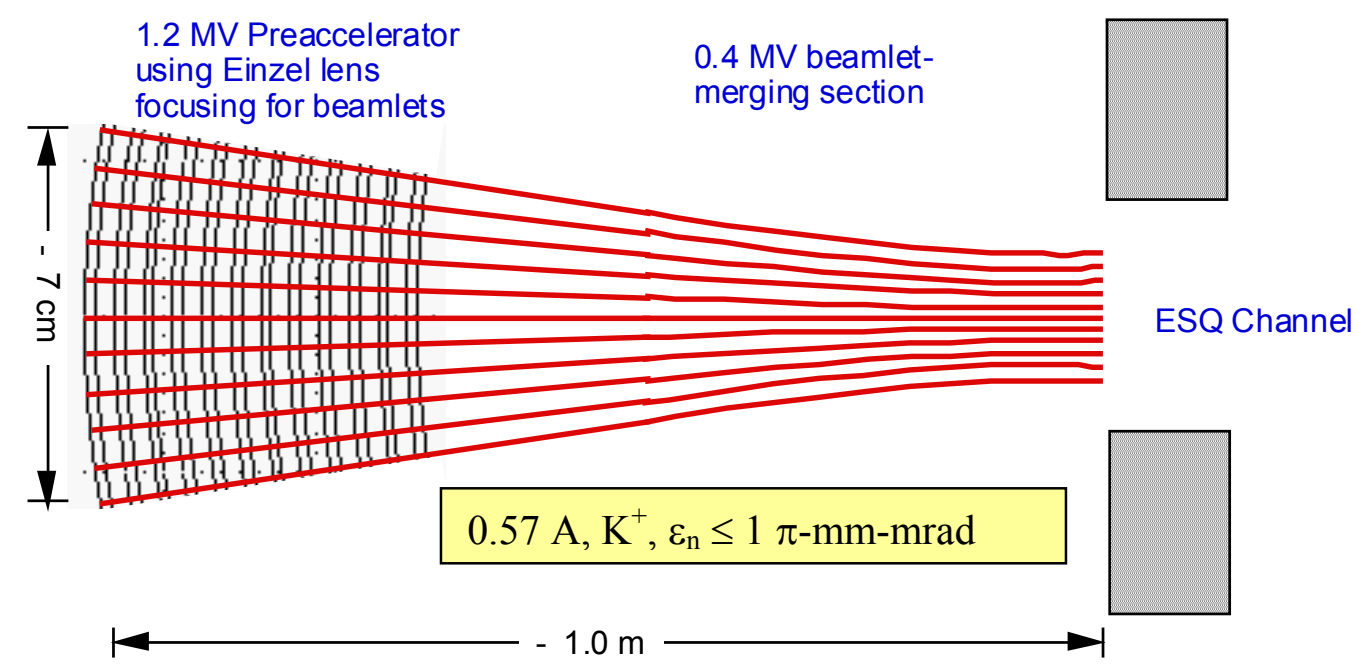

Figure 1.2 Detail of the dimensions of a multiple-beamlet injector. 


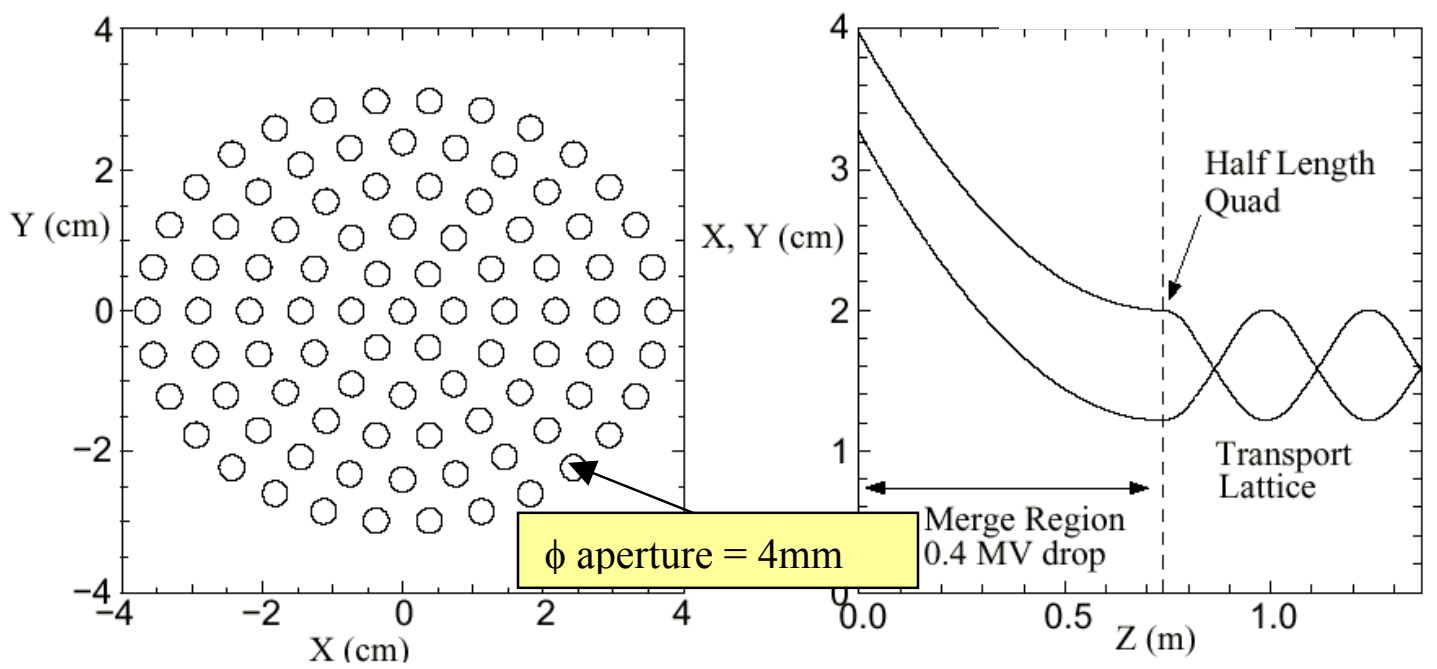

Figure 1.3. Aperture configuration at the last accelerating plate (left) and $X$ and $Y$ beam radii on merge region and entrance to the transport lattice.

With the multiple beamlet architecture, a concern that arises is that of emittance growth due to the required merging process. Previous work in this problem [Anderson] indicates that free space charge field energy leads to emittance growth. Here free energy is defined by the difference between the self-field energy of a specific configuration and that of a single, uniform beam with the same perveance and root-mean-square radius. Free energy will be available whenever the space charge distribution is non-uniform, which is an inherent characteristic of multiple beamlet merging.

Simulations have shown that the emittance growth after the merging process can be reduced through different measures: a) increasing the merging energy, b) decreasing the inter-beamlet spacing and c) increasing the number of beamlets.

Following these guidelines, a basic design (Grote, 2002) for an HIF injector would consist of the initial accelerating columns leading into a merging region. 
Furthermore, since the individual beamlets can be properly aimed, the system can be designed such that the merged beam is matched when it enters the transport channel. The elimination of a matching section is a significant advantage over traditional injector architectures where the required matching of a round beam to an elliptical transport channel usually requires large envelope excursions and thus larger system volume.

The simplest design for the initial accelerating region consists of a series of alternating gradient Einzel lenses. The alternating potential between the plates provides an alternating focusing and defocusing effect that transports the beam through the system. This type of beam transport only is effective at low energies. Since it is desirable to do the merging at as high energy as possible, a compromise has to be attained.

The inter-beamlet spacing can not be arbitrarily reduced since there are material requirements between the apertures to provide mechanical support. Aperture offsets are important since the transverse force on the beamlets due to offsets could steer them into the metal plates. The plates must also have small separation between them (relative to the aperture radii and beamlet separations) so that the electrostatic forces between the beamlets are minimized. For a given beamlet current, the size is constrained by the voltage differences between the plates, the aperture radius and the beam energy.

The final conditions of the merging section are dictated by the requirements of the transport channel (current, energy, beam size and divergence). This in turn determines the overall length of the merging section as well as the beam RMS convergence at the start of the region. With these constraints, the main free parameters in the design become the number of beamlets and the merging energy. The transport channel current together with the number of beamlets determines the current per beamlet. 
The Einzel lenses focusing strength, as well as the maximum current density obtainable from the used ion source, constrain the source emitter diameter for the given current per beamlet. The system is further determined by defining the clearance between the beamlets and the corresponding apertures and the inter-aperture spacing. 


\section{Chapter 2: Potassium aluminum silicate ion sources}

\subsection{On the structure and nomenclature of the alkali aluminosilicates.}

In order to have a clearer idea of the nature of the aluminosilicate sources, some background in mineralogy is justified. This becomes particularly important since the literature on diverse ion emitting minerals is usually confusing regarding the names of the used minerals, making terms like "zeolites", "aluminosilicates" and more specific ones like "eucryptite" or "spodumene" fully interchangeable. This section is a modest effort to clarify the relevant nomenclature.

The alkali aluminosilicates belong to the largest class of minerals, the silicates. The basic building block of this class is the $\mathrm{SiO}_{4}$ group, a tetrahedron structure with $\mathrm{a}-4$ charge. 


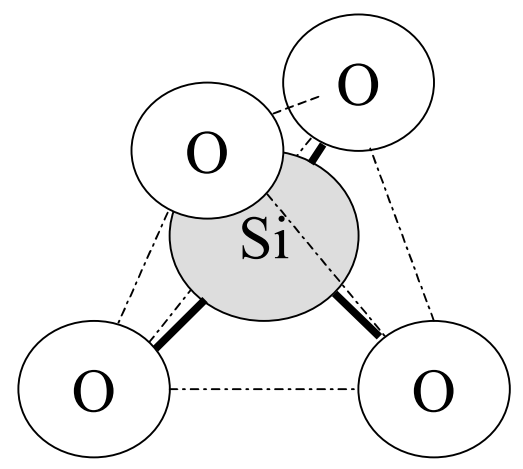

Figure 2.1. $\mathrm{SiO}_{4}$ group, elementary building block of the silicates.

The +4 valence of the $\mathrm{Si}$ atom is paired to one valence electron in each oxygen atom, leaving every oxygen free to form an additional bond with other atoms, thus leaving the net valence of -4 .

The silicates are divided in turn by subclasses, not by their chemistry, but by their structure. The classification is shown in Figure 2.2. Though not exhaustive, in the sense that not all the subclasses are listed, this classification is a convenient way of picturing how the minerals of interest for the present application relate to one another.

Of particular interest are minerals in the tectosilicate subclass. These "framework silicates" are composed of interconnected tetrahedrons yielding a silicon-to-oxygen ratio of 1:2. In pure form, when only $\mathrm{Si}$ and $\mathrm{O}$ are present, this mineral is known as quartz. However, it is possible for Aluminum ions to replace silicon ions up to 50\%. The amount of aluminum present is the main cause for the structural variations in the tectosilicates. While the tetrahedral structure remains the same, the net valence of the system becomes 5 (since the oxidation state of the $\mathrm{Al}$ is +3 ). To preserve charge neutrality in the crystal, additional cations are needed in the overall structure to preserve the charge neutrality. This is the main source of variations within this subclass. 
It is within the tectosilicates that we find the minerals of interest for ion source applications, in particular, some types of potassium aluminum silicates. The potassium aluminum silicates are present primarily as the alkali feldspars listed in Figure 2.2. The general formula for the common feldspars is $\mathrm{XAl}_{(1-2)} \mathrm{Si}_{(3-2)} \mathrm{O}_{8}$, where the $\mathrm{X}$ can be sodium, potassium or calcium, or a combination of these. When the cation represented by $\mathrm{X}$ has a +1 charge, as it would in the case of $\mathrm{Na}$ or $\mathrm{K}$, then the formula will contain one $\mathrm{Al}$ ion and three Si ions. On the other hand, if the valence were +2 (as with calcium), then the formula will contain two aluminum and two silicon ions. This keeps the formula balanced as aluminum has a charge of +3 while silicon has one of +4 . This is an interesting example of how the composition of the mineral directly relates with the conditions of charge neutrality and the conditions that may or may not favor the emission of ions from such structure. The potassium feldspars listed in Figure 2.2 are examples of potassium aluminum silicates, like the microcline and orthoclase, (sanidine is a potassium sodium aluminum silicate), but none of these minerals is actually used in ion source applications.

The feldspathoid group the most important for our application. The feldspathoids are not typical in the sense that they are not related to each other by structure and chemistry, but by their particular relation with the feldspars. The feldspathoids are low silica igneous minerals that would have formed feldspars if the original magma that formed them had been richer in silica. While in the feldspars the aluminum to silicon ratio is $1: 3$, in the case of the feldspathoids this ratio is nearly $1: 1$.

The feldspathoids are similar to the zeolites in that they have large openings within the crystal structure. These openings are mostly separated from each other and do 
not lend themselves to the movement of ions and molecules that is possible in the larger openings of the zeolite structure. Among the feldspathoids, the only potassium aluminum silicate goes by the name of leucite, and has the general formula $\mathrm{K}_{2} 0 \mathrm{Al}_{2} \mathrm{O}_{3} \cdot 4 \mathrm{SiO}_{2}$. This last mineral is the one used throughout the experiments described in this work.

The name "leucite" has its etymological origin in the Greek word for "white", in direct allusion to its typical color. At temperatures higher than $605{ }^{\circ} \mathrm{C}$, leucite is isometric and will form a trapezohedron crystal. At lower temperatures, the isometric structure is unstable and transforms into a tetragonal structure without altering the outward shape. The following is a list of some of leucite's physical characteristics.

a. Color: clear, white or gray, with possible yellowish and reddish tints.

b. Luster: vitreous, greasy or dull.

c. Transparency: the crystals are transparent, translucent to commonly opaque.

d. Cleavage: absent.

e. Hardness: $5.5-5$.

f. Specific Gravity: $2.4-2.5$.

g. Streak: white.

These characteristics correspond to what is observed in the samples used throughout HIF experiments.

As information of interest, Figures 2.3, 2.4 and 2.5 show the phase diagram relevant for understanding the diverse compositions and temperatures of the $\mathrm{K}_{2} \mathrm{O}-\mathrm{Al}_{2} \mathrm{O}_{3}$ - $\mathrm{SiO}_{2}$ system, and the structural diagram of the leucite phase at temperatures below 605 ${ }^{\circ} \mathrm{C}$. The tetrahedral disposition of oxygen atoms with a $\mathrm{Si}$ or $\mathrm{Al}$ atom at its center can be 
seen. The larger (green colored) atoms correspond to the position of the alkali in the compound.

Regarding some other common terms that appear on the literature, the main distinction that is usually made is regarding the difference between spodumene, a mineral of the form $\mathrm{X}_{2} \mathrm{O} \cdot \mathrm{Al}_{2} \mathrm{O}_{3} \cdot 4 \mathrm{SiO}_{2}$, and eucryptite, of the form $\mathrm{X}_{2} \mathrm{O} \cdot \mathrm{Al}_{2} \mathrm{O}_{3} \cdot 2 \mathrm{SiO}_{2}$, where $\mathrm{X}$ represents the alkali metal of interest. It appears that the terms "spodumene" and "eucryptite" should apply only when the used alkali is lithium. In our case, Leucite would be the corresponding spodumene-like compound. Depending on the application and the type of ion required, one of the previous two types of compounds may be preferable to the other. The literature mentions in general that when the required ion is lithium, the eucryptite is a preferrable material. In case that sodium and or potassium are required, the "spodumene-analog" (that is, leucite) is the material preferred at temperatures above $1,000{ }^{\circ} \mathrm{C}$. This is also the case for rubidium, although it is mentioned that higher temperatures are required in this last case, above $1,100{ }^{\circ} \mathrm{C}$.

Another term that seems to be generically used is the term "zeolite". Zeolites are framework silicates consisting also of interlocking tetrahedrons of $\mathrm{SiO}_{4}$ and $\mathrm{AlO}_{4}$. The zeolites have large vacant spaces in their negatively charged structure. These spaces allow large cations, molecules and cation groups to reside and move throughout the structure. While the literature offers examples of zeolites used as ion emitters, such as the case of mordenite $\left(\left(\mathrm{Ca}, \mathrm{Na}_{2}, \mathrm{~K}_{2}\right) \mathrm{Al}_{2} \mathrm{Si}_{10} \mathrm{O}_{24} \cdot 7 \mathrm{H}_{2} 0\right)$, a hydrated calcium, sodium, potassium aluminum silicate, these have not been known to have better performance or ion output than the minerals currently in use in our applications and so have not been used in the present work. Preparation of suitable ion emitting zeolites would include 
some kind of ion-substitution process which is not $100 \%$ efficient, thus leading to an inherent impurity content.

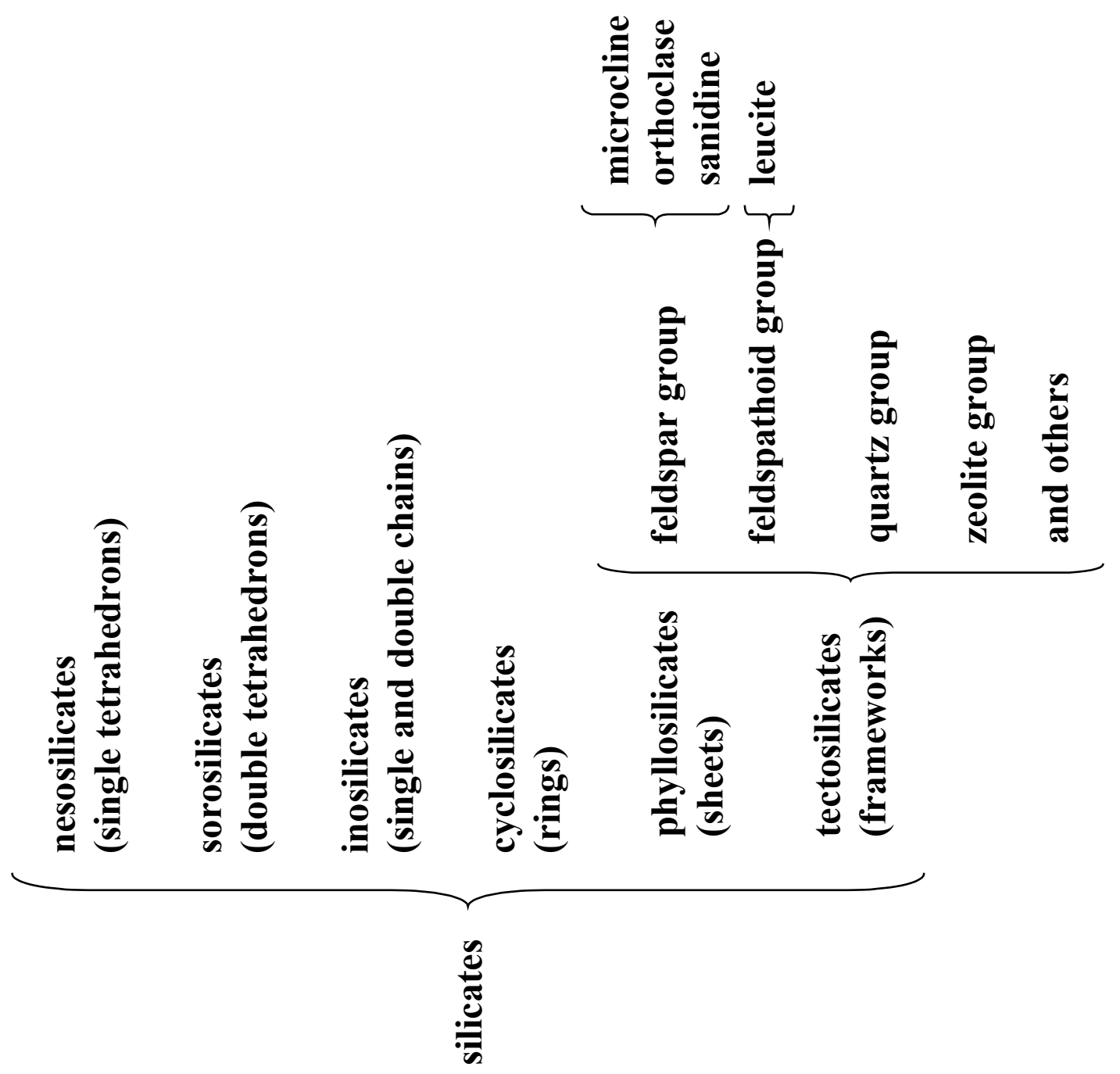

Figure 2.2. Clasification of the silicates by structure. 


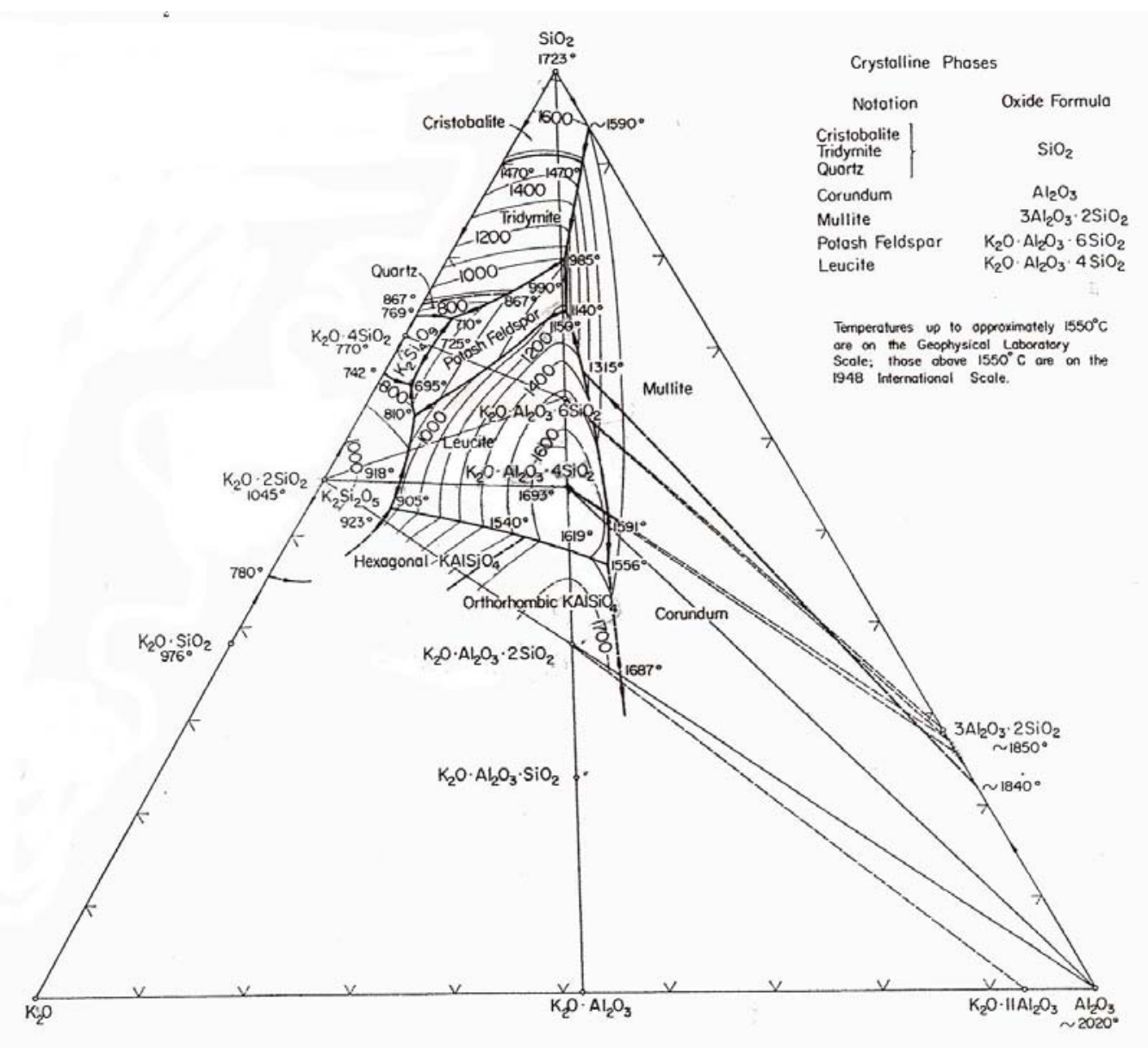

FIG. 407.- System $\mathrm{K}_{2} \mathrm{O}-\mathrm{Al}_{2} \mathrm{O}_{3}-\mathrm{SiO}_{2}$; composite.

E. F. Osborn and Arnulf Muan, revised and redrawn "Phase Equilibrium Diagrams of Oxide Systerns," Plate 5, Published by the American Ceramic Society and the Edward Orton, Jr., Ceramic Foundation, 1960.

Figure 2.3. Phase diagram of the $\mathrm{K}_{2} \mathrm{O}-\mathrm{Al}_{2} \mathrm{O}_{3}-\mathrm{SiO}_{2}$ system. 


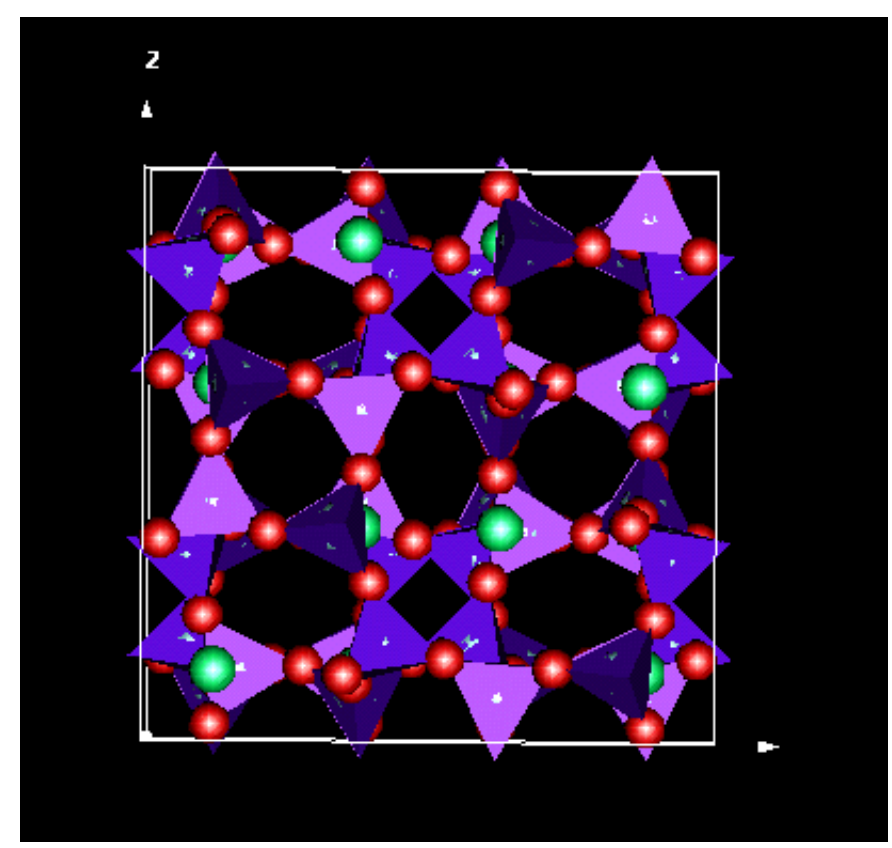

Figure 2.4 Structure of the mineral leucite in its low temperature form. Color Code: Green: K, Red: O, Al and Si occupy the space inside the purple tetrahedrons.

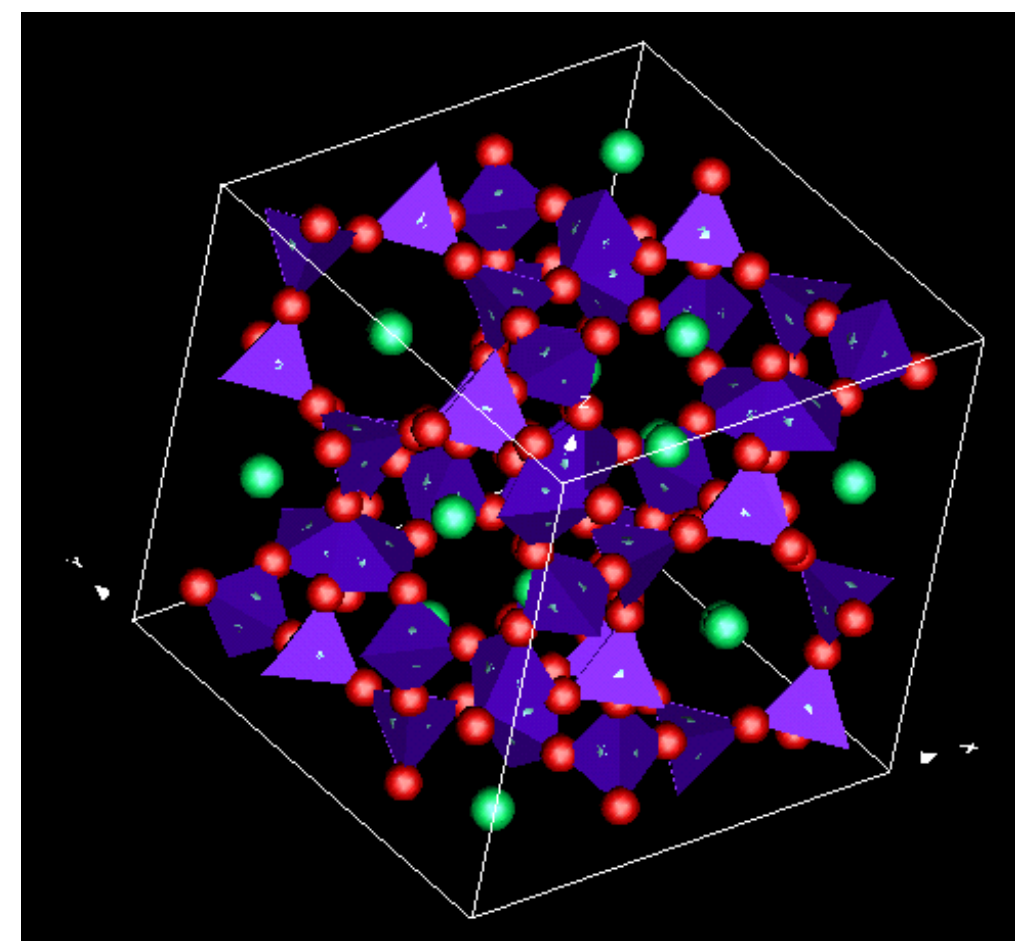

Figure 2.5. Alternative view of the leucite structure revealing the apertures in the structure. Same color coding as in Figure 2.4. 


\subsection{Scanning electron microscope studies on Aluminosilicates.}

A goal of the studies carried out in this work regarding aluminosilicates has been the introduction of more objective criteria regarding the characteristics and quality of the emitter materials used for HIF's ion sources. This need arose due to variation in the emitting properties observed in aluminosilicates prepared with what were thought to be equal procedures. To attain this objective, a more systematic use of a Scanning Electron Microscope (SEM) in the analyses of the aluminosilicates was introduced. The goals of this effort have been to identify how the types of microscopic structures relate to emission quality, to explore what types of elemental composition relate to acceptable emission properties and to verify if any changes were needed in the standard alkali aluminosilicate fabrication procedures.

These types of analyses were made not only on the sources intended for high current density yield, but also in large (multi-cm) diameter sources used in diverse HIF experiments. So among the outcomes of this effort one can find a quality control technique for the alkali aluminosilicates prepared for HIF.

\subsubsection{Preparation of alkali aluminosilicates.}

The standard way of preparing an alkali aluminosilicate source is described in internal documentation in the HIF group (Chupp, 1993) which in turn has been adapted from the literature (Feeney, 1976). As a summary, the alkali aluminosilicates are prepared by mixing stoichiometric quantities of an alkali carbonate. The ones used in our experiments are synthesized by mixing stoichiometric quantities of an alkali carbonate, 
silicon oxide and aluminum oxide. Significant care must be taken regarding the humidity control and thorough mixing. Equation (2.1) represents the aluminosilicate forming reaction. X represents the alkali species.

$$
\mathrm{X}_{2} \mathrm{CO}_{3}+\mathrm{Al}_{2} \mathrm{O}_{3}+4 \mathrm{SiO}_{2} \longrightarrow \mathrm{\Delta}_{2} \mathrm{O} \cdot \mathrm{Al}_{2} \mathrm{O}_{3} \cdot 4 \mathrm{SiO}_{2}+\mathrm{CO}_{2} \uparrow
$$

The product is ground until it can be sifted through a 200 -mesh wire sieve. A slurry, made of aluminosilicate powder and de-ionized water is poured onto the surface of the tungsten substrate. In case of large ion sources, a plastic molding tool, is used to press the slurry and give it its final form with a desired thickness $(\sim 0.5 \mathrm{~mm})$. Slow drying is carried out in a chamber with humidity control. The final step is firing of the coated substrate at $1,580^{\circ} \mathrm{C}$ in a vacuum furnace. The temperature profile is controlled for both heating and cooling at a rate of $\sim 10^{\circ} \mathrm{C} / \mathrm{min}$ at temperatures above $700{ }^{\circ} \mathrm{C}$. Inadequate temperature control, or excessive temperature (above melting) may result in undesired loss of cations in the compound with subsequent change in the composition of the material, or surface distortion due to the molten compound's surface tension.

The aluminosilicate powder composition used to make the sources was analyzed using the X-ray characteristics resulting from an incident electron beam in the SEM. The results obtained form the basic signature of the aluminosilicate under study. The following Figure 2.6 shows such a characteristic signature. The vertical axis shows the number of counts detected at each energy bin. The spectrum shown, as well as similar spectra obtained for raw aluminosilicate powders have been used throughout the experiments as a benchmark against which variations in composition are assessed. It is important to note that in relative terms, the signals obtained for 3 of the basic elements, 
oxygen, aluminum and potassium are of roughly the same intensity, and all of them are between 40 to $70 \%$ of the intensity of the silicon line. Significant variations of the composition will be observed depending on the preparation characteristics of the studied samples. These will be shown below.

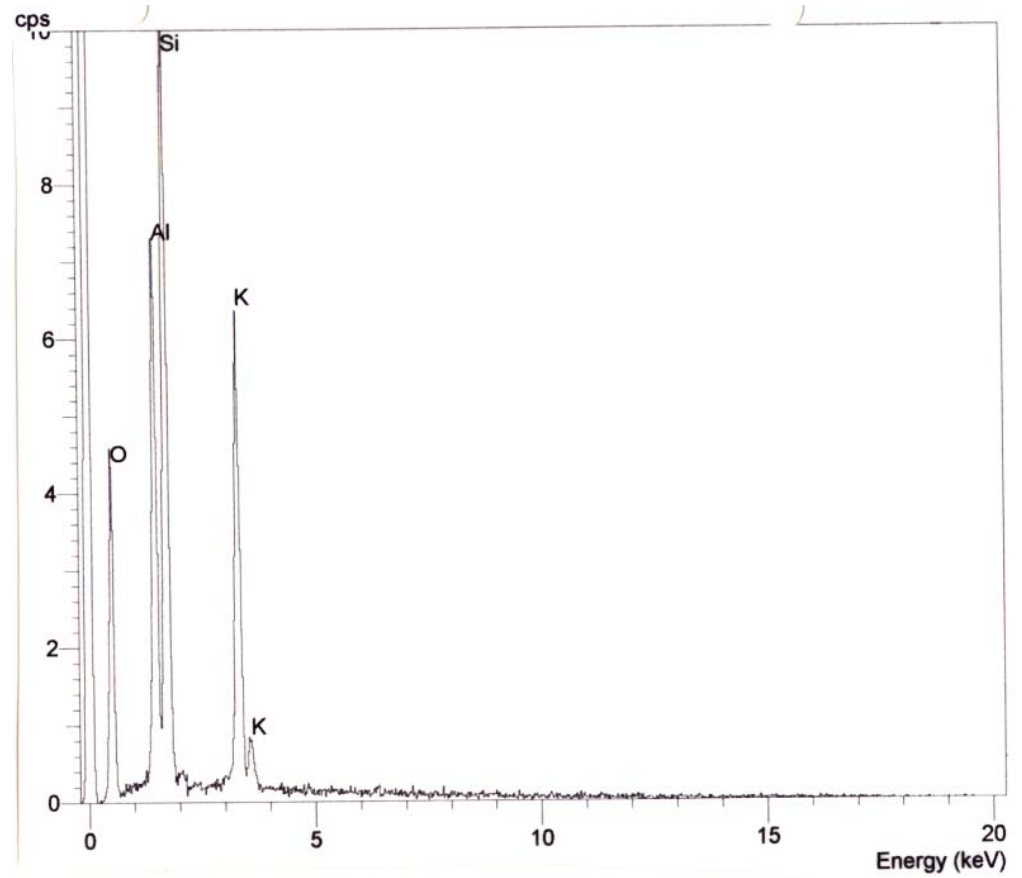

Figure 2.6. Scanning Electron Microscope $\mathrm{X}$ - ray signature of a potassium aluminosilicate powder.

With the SEM tool, it is possible to identify the spatial location of the signals observed in the composition spectra. This has proven to be an important tool, since the presence of all the elements required to form the aluminosilicate does not necessarily imply having the right compound. Figure 2.7 shows a set of such maps. When the electron beam of the SEM strikes the material to be analyzed, electrons in the materialk go to excited states. When these electrons return to their ground states they release characteristic photons which are registered by the detector. The information from the 
detector is then processed and correlated with the information in an element library that contains the expected energy of the photons, by element, at the electron beam energy used. Images like the one shown in Figure 2.7 represent the intensity of the photon count as a function of the position, thus giving an idea of the element distribution. It is possible to observe a positive correlation among all the components (the signals from the elements come in general from the same regions), as would be expected from a potassium aluminosilicate mineral. It should be remembered that at the scale that the SEM is analyzing, there should not be any possibility of discerning the different positions of the atoms on any element if the structure is an aluminosilicate mineral. Any separation of the elements should thus be an indication that fundamental deviations from the aluminosilicate structure are occurring.

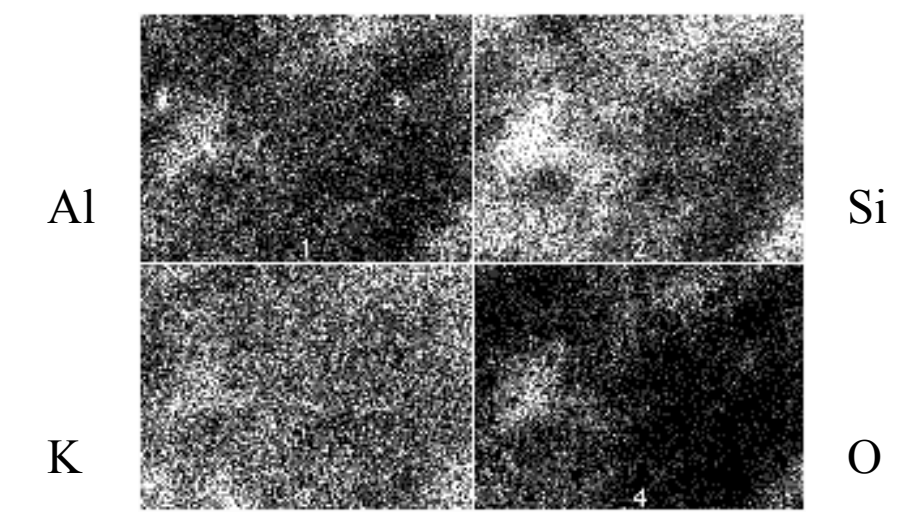

Figure 2.7. Intensity map on an element basis. The width of each of these maps represents a length of $\sim 24 \mu \mathrm{m}$ in the original sample.

The presence of impurities has been an additional issue addressed with these diagnostics. Figure 2.8 shows a spectrum of a potassium aluminosilicate powder that was prepared with an insufficiently pure aluminum oxide compound. Note the detection of a 
small zirconium peak. In general, purity levels of $99.99 \%$ for the compounds are recommended.

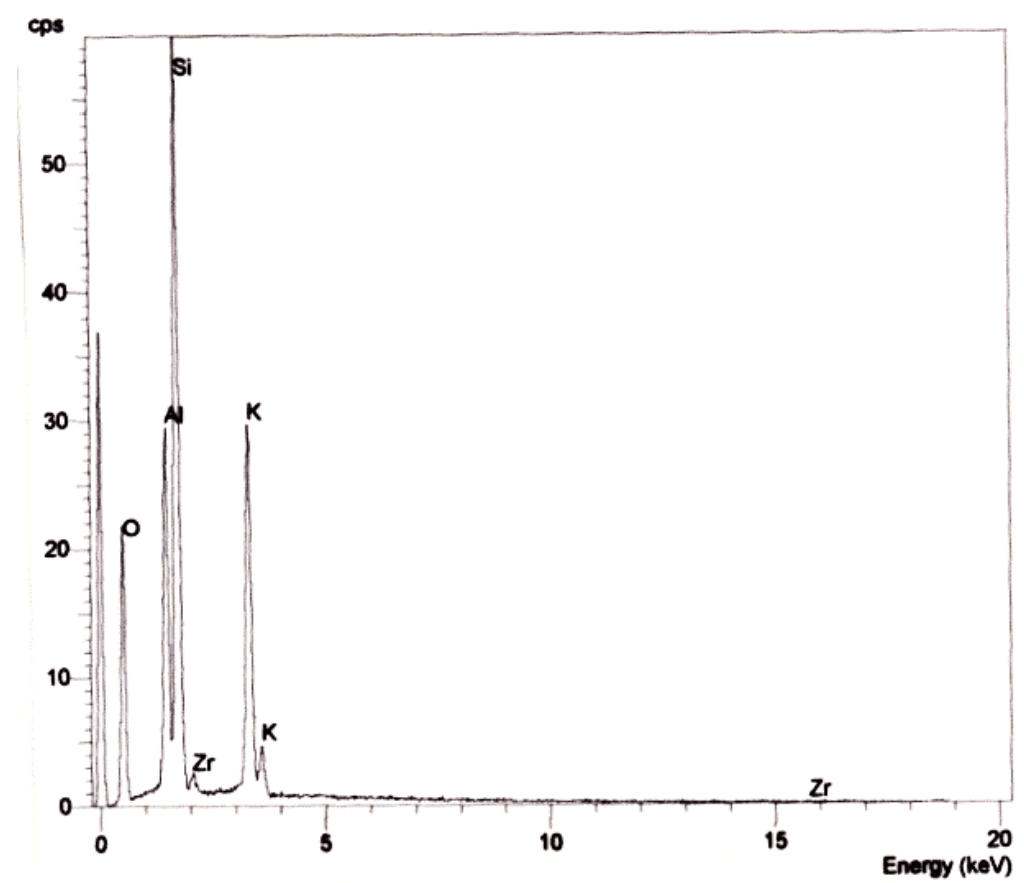

Figure 2.8. Potassium aluminosilicate spectrum depicting a zirconium impurity.

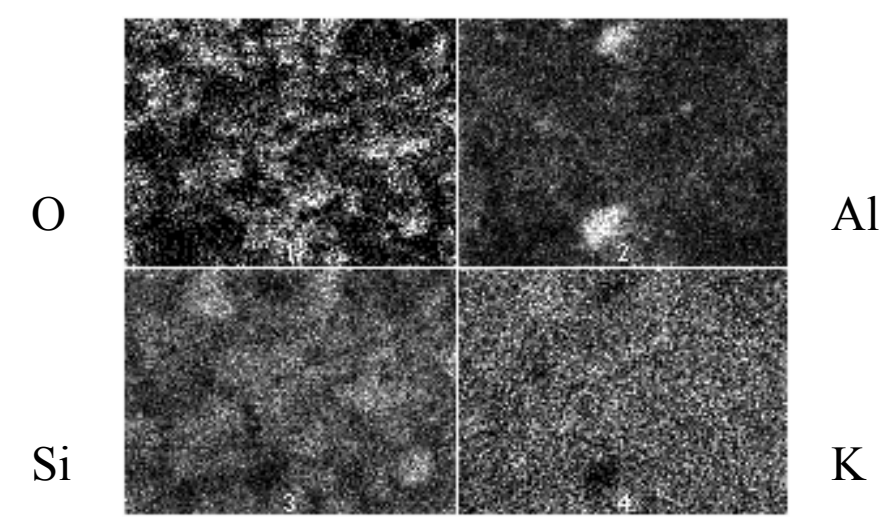

Figure 2.9. Element distribution map corresponding to Fig. 2.8. Note again positive correlation among the elements. Higher intensity spots on the Al seem to be aluminum oxide specs on the surface. Horizontal width of each map corresponds to a length of $\sim 120 \mu \mathrm{m}$ in the original surface. 


\subsubsection{Element composition dependence on preparation temperature.}

Despite the ubiquitousness of the potassium aluminosilicate mineral in HIF experiments, the knowledge base of this material has been developed traditionally in an empirical way. This has been the tradition regarding the most appropriate methods for preparation of the mineral itself and then the ion sources.

The effort undertaken in this section depicts the first attempts within the HIF practice, to the author's knowledge, to observe changes in composition of the material, as a function of the preparation conditions. As the next results show, the composition and microscopic appearance of the ion sources depends very strongly on the preparation temperature. This can be easily seen in the next figures showing the composition of several $1 / 4$ " ion sources, prepared with the same base powder and metallic substrates. The only difference has been the heating temperature.

Figure 2.10 shows the spectrum of an aluminosilicate source fired at $1,500{ }^{\circ} \mathrm{C}$ in an argon atmosphere. Figure 2.11 shows the corresponding spatial distribution of the elements from the area analyzed on Figure 2.10. It is worth noting at this point that the use of noble gas atmospheres during firing was avoided due to the very poor performance of the ion sources prepared with this method. Vacuum conditions are preferred. In general, it was observed that when argon atmospheres were used, there was a negligible ion output by the emitter. Furthermore, the inspection of the surface appearance after firing the emitter in the ion gun revealed bubble-like structures at the surface as if gas had been released during firing. It is unclear why there was such effect by the argon atmosphere, but given the undesirable results, it was concluded that vacuum atmospheres would subsequently be used. 


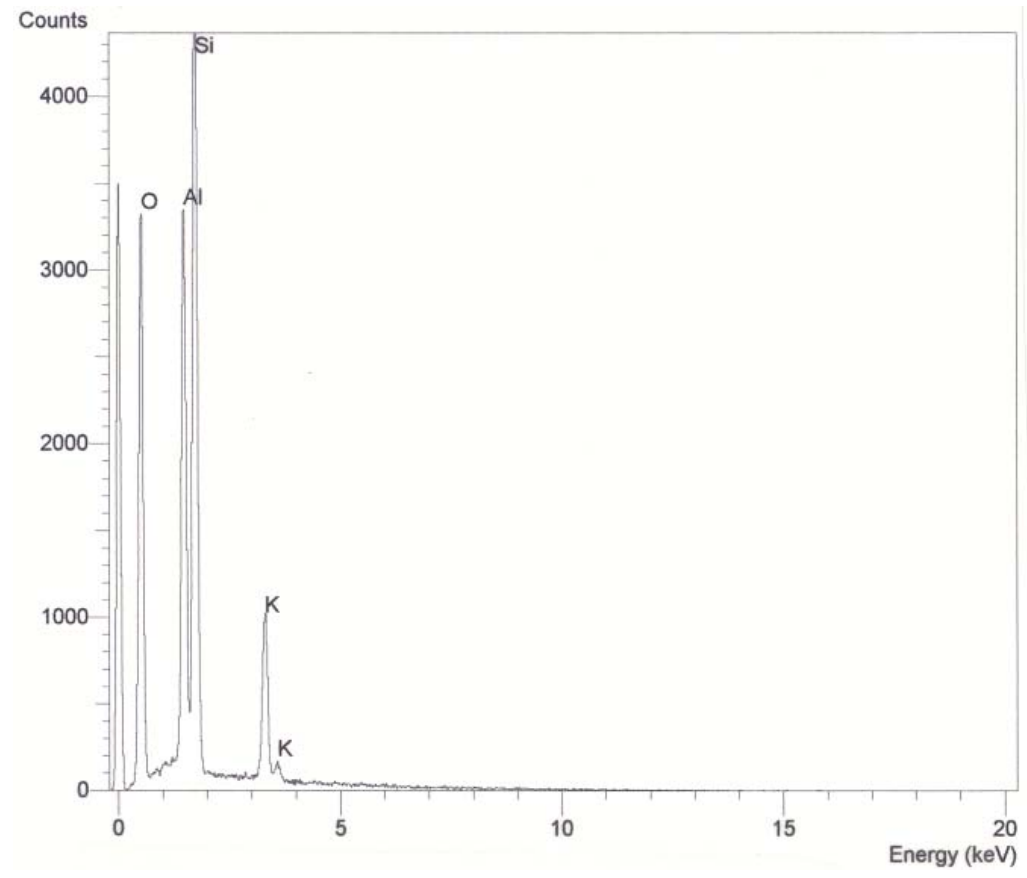

Figure 2.10. Spectrum of an aluminosilicate source prepared at $1500^{\circ} \mathrm{C}$ in an argon atmosphere. Glassy appearance with specs of aluminum oxide.

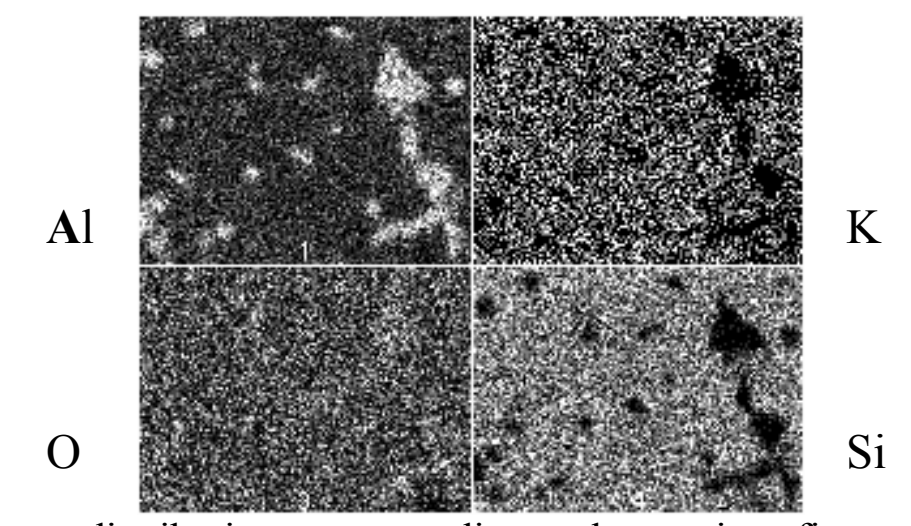

Figure 2.11. Element distribution corresponding to the previous figure. Horizontal length in each map corresponds to $\sim 30 \mu \mathrm{m}$ in the original surface. Note again high positive correlation among the elements except for the aluminum oxide specs.

Figure 2.12 shows a spectrum of an aluminosilicate emitter prepared at $1,550{ }^{\circ} \mathrm{C}$.

Note the decrease in the relative potassium content with respect to the lower firing temperature presented in Figure 2.10. While the surface in this figure had a glassy appearance with specs of aluminum oxide, the analyzed region did not contain specs. 
Hence a more accurate assessment of the Al value is obtained (otherwise the Al signal would be higher and closer to the Si peak).

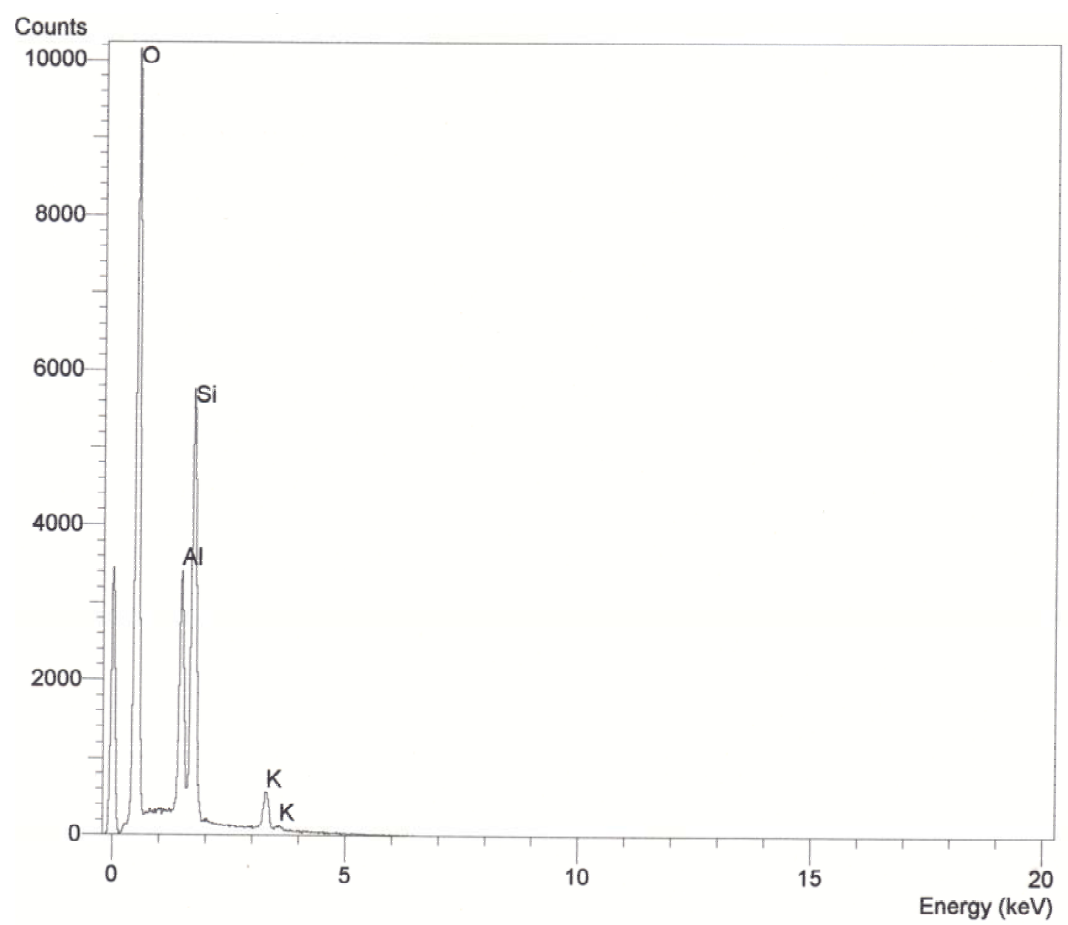

Figure 2.12. Spectrum of an aluminosilicate source prepared at $1550^{\circ} \mathrm{C}$ in an argon atmosphere.

Figure 2.13 shows a photograph of the region analyzed on the previous figure. Note the glassy appearance of the surface with the specs of aluminum oxide. Figure 2.14 shows the corresponding element distribution maps. The image corresponds to the central part of the photograph in Figure 2.13. 


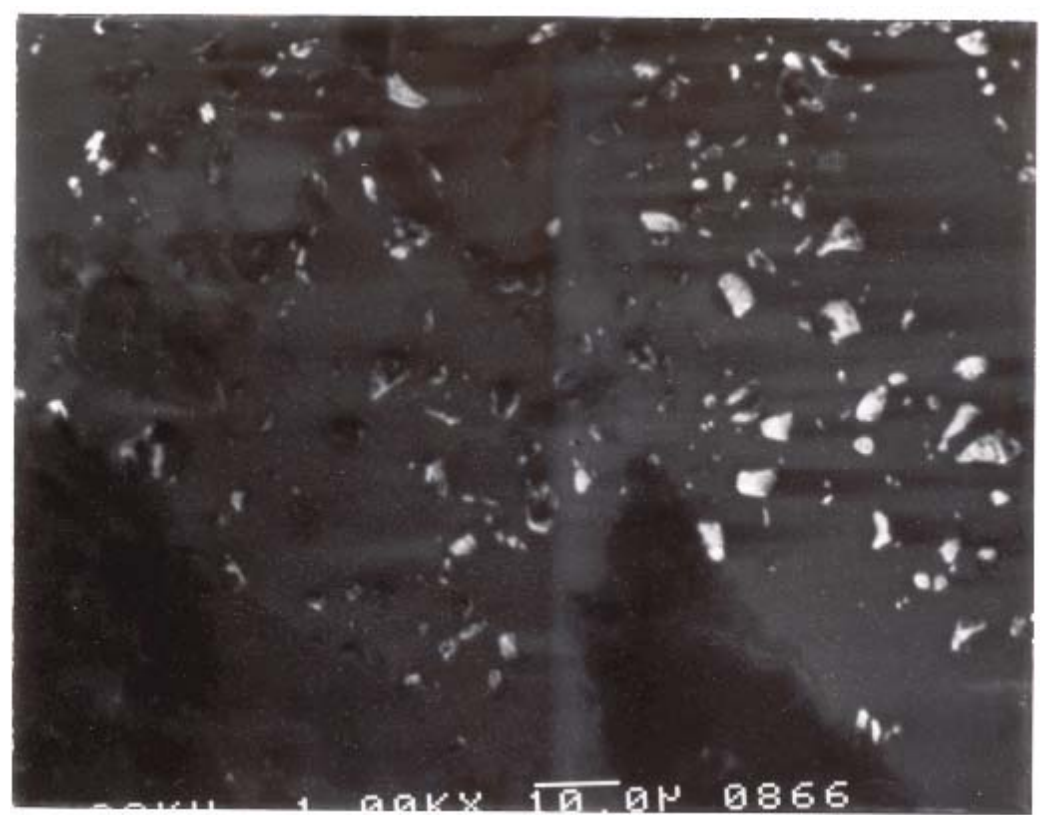

Figure 2.13. Photograph of the aluminosilicate prepared at $1,550^{\circ} \mathrm{C}$. Note the aluminum oxide specs on the surface. 1,000X magnification.

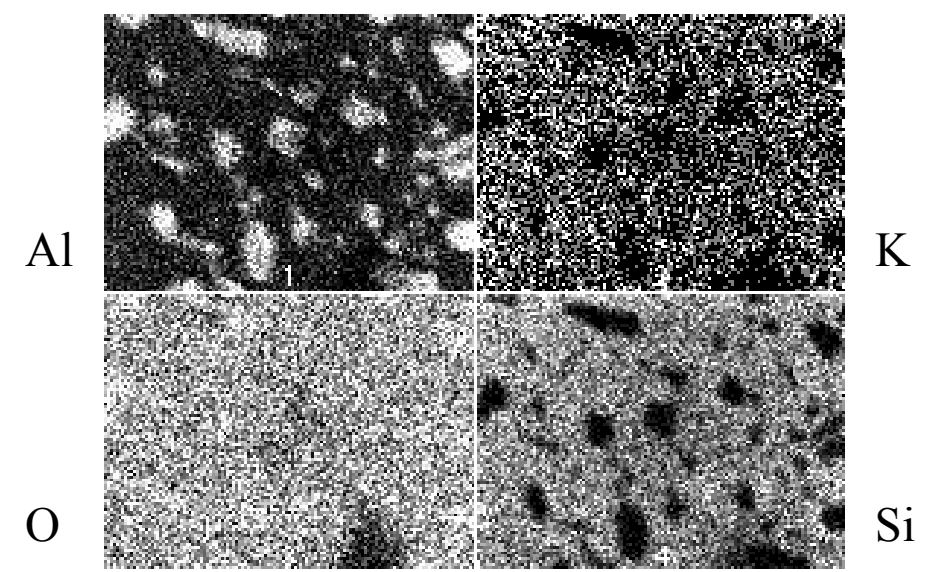

Figure 2.14. Element distribution of the aluminosilicate prepared at $1,550^{\circ} \mathrm{C}$. Image corresponds to the very central part of the previous photograph.

Figure 2.15 displays the spectrum of an aluminosilicate prepared under yet higher temperatures, in this case, $1,610^{\circ} \mathrm{C}$. Note the further relative decrease in the strength of the potassium peak. It is around this temperature that the components of the aluminosilicate compound start separating from each other and different crystal forms 
begin to emerge. Specifically, a needle-like structure composed of aluminum oxide crystals starts to become visible. This is more clearly shown in Figure 2.16, the corresponding spatial distribution map. A more dramatic case is shown in figures 2.17 and 2.18, of an emitter fired at an even higher temperature of $1,660^{\circ} \mathrm{C}$.

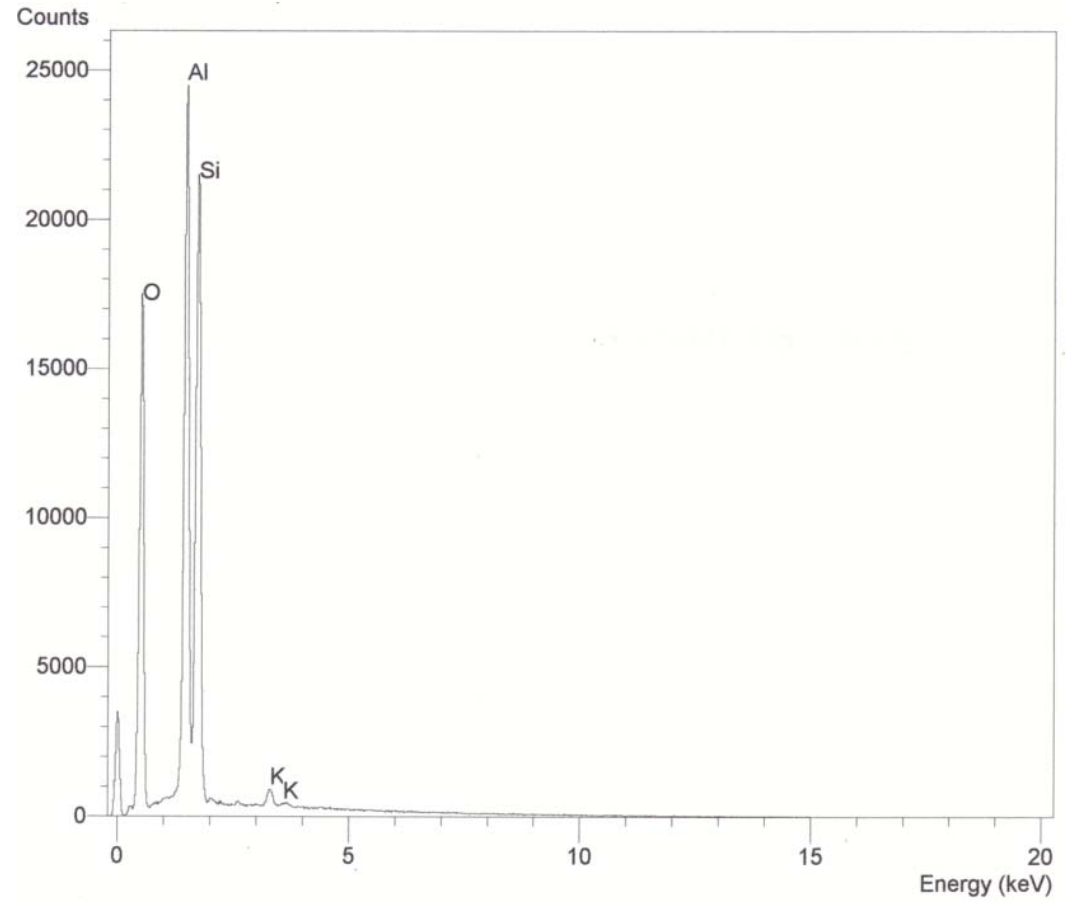

Figure 2.15. Spectrum of an aluminosilicate source prepared at $1,610^{\circ} \mathrm{C}$ in vacuum. Note the significant decrease of potassium. 


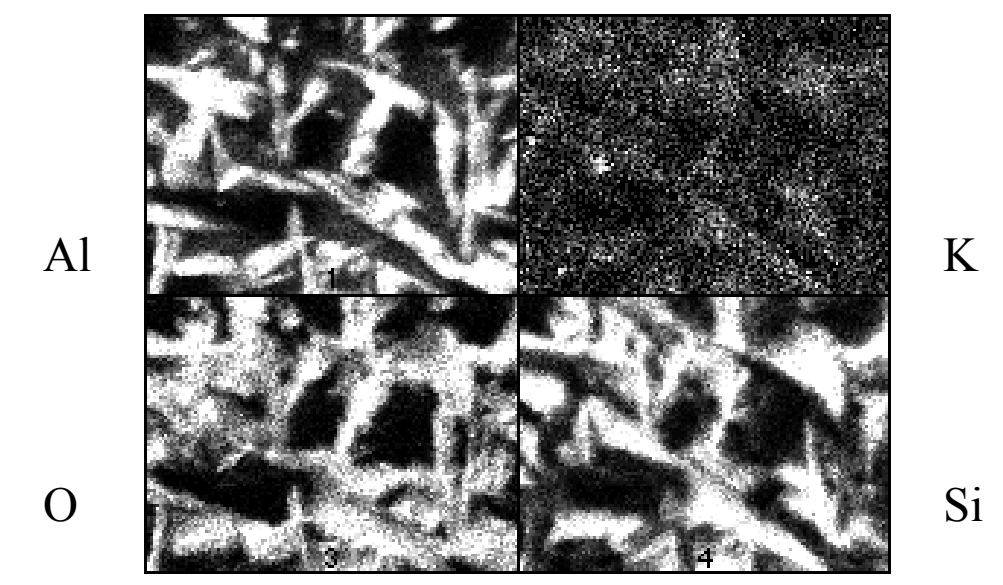

Figure 2.16. Element distribution map of the aluminosilicate prepared at $1,610^{\circ} \mathrm{C}$. There is evidence of some degree of separation between $\mathrm{Al}$ and $\mathrm{Si}$ at this level.

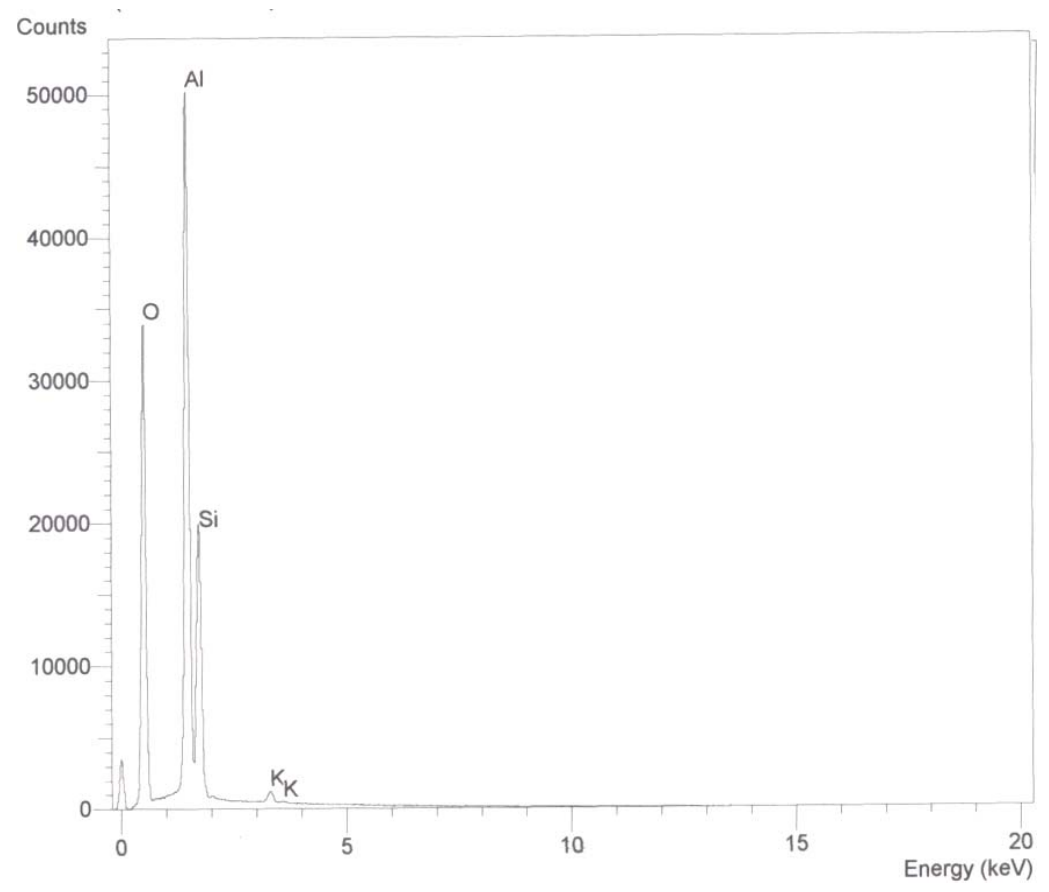

Figure 2.17. Spectrum of an aluminosilicate source prepared at $1,660^{\circ} \mathrm{C}$ in an argon atmosphere. Note the significant decrease of potassium. 


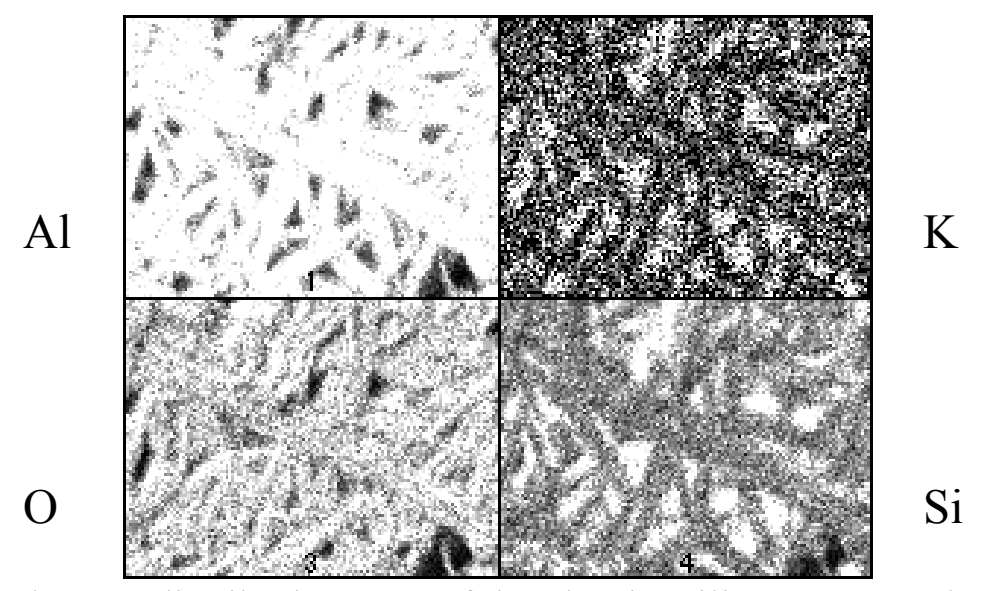

Figure 2.18. Element distribution map of the aluminosilicate prepared at $1,660^{\circ} \mathrm{C}$. At this temperature, there is a significant separation of the components. Silicon and aluminum are now anti-correlated. The little potassium that remains is associated with the $\mathrm{Si}$ while the dendrites appear to be formed out of aluminum oxide.

From the above discussion, it seems that the most important consideration in the preparation of the aluminosilicate is to maintain a preparation temperature that will preserve the physical integrity of the desired material. It is clear that at temperatures around $1,600{ }^{\circ} \mathrm{C}$ and above, there are obvious changes in the structure of the material as seen above. These changes have been manifested in a change of the material composition and a more serious separation of the components (elements). These changes are known to happen even at lower temperatures (above $1,100^{\circ} \mathrm{C}$ ) but over much longer periods of time (weeks as opposed to hours). This will be shown at the end of Section 4.2.3. where composition spectra are shown for an operational, high ion yielding aluminosilicate source. In that section it is shown how the total potassium content of the material seems to be a less important factor for the capability to deliver high current densities than the maintenance of a uniform element composition. Section 4.2.3. presents is information to support the claim that the improvement in the ion output capability of this type of emitter may be related with the decrease of potassium with time. 
For instance, compare the composition spectra shown in Figure 2.12 (equivalent to the "before" picture) with what can be seen in Figures 4.43 and 4.51 (the "after" pictures). The comparison is not ideal since the operational emitters were prepared in a vacuum atmosphere and a firing temperature around $1,575{ }^{\circ} \mathrm{C}$, while Figure 2.12 was prepared in an argon atmosphere. However, a general idea of the expected change in potassium content can be obtained with this comparison.

As a last note, it is important to emphasize how the only positive experimental results were obtained with vacuum atmospheres, and not with noble gases, which are the usual alternative. 


\section{Chapter 3: Experimental Setup}

This section describes the experimental facility and the relevant equipment used throughout these experiments.

\subsection{Experimental Test Stand}

The main experimental facility used was a small test stand intended for ion source development work. The system is comprised of a main cylindrical vacuum tank with multiple equipment and diagnostic ports on the circumference and a high voltage insulated column on the back of the tank to feed the required electric potentials to the ion source.

Operating pressures inside the tank as low as $2 \cdot 10^{-7}$ torr were achieved by the dual action of a turbomolecular pump and a roughing pump. A cold cathode gauge monitored the base gas pressure. 
Figure 3.1 shows a photograph of the vacuum tank used in these experiments.

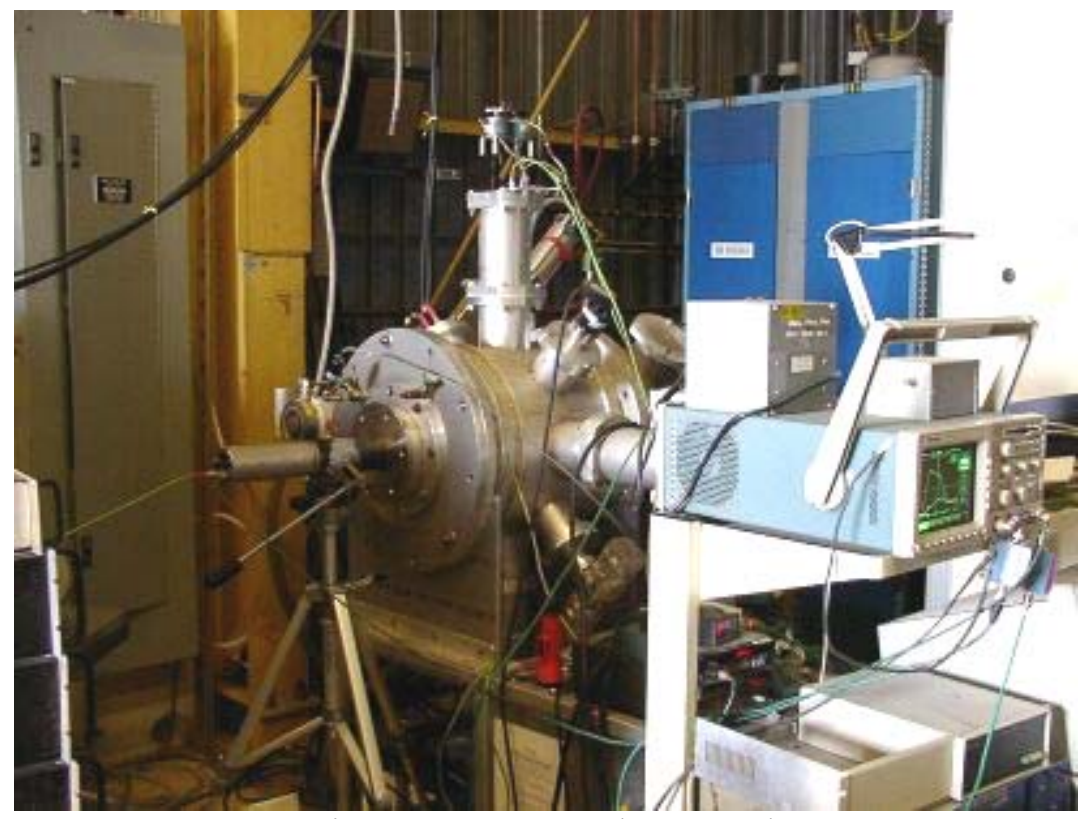

Figure 3.1 Setup Photograph

There were two main sources of high voltage for these experiments. The most widely used one was a bi-polar, $\pm 35 \mathrm{kV}$ maximum output, high voltage supply based on a thyratron-triggered pulse-forming-network. This power supply provided the highest electric field gradients achievable in these experiments and was used to test for maximum current output of the ion sources. The maximum pulse length achievable with this machine was $\cong 2.5 \mu \mathrm{s}$. The second source of high voltage was a solid-state modulated $+50 \mathrm{kV}$ nominal, $20 \mu$ s nominal, monopolar power supply. This machine was mostly used to test for the current yielding capability of the ion sources at longer extraction pulse lengths. However, given the impedance characteristics of the setup, there was a trade-off between the capability to obtain the ideal square pulse and the capability to obtain the full voltage. Therefore, in order to obtain the highest voltages possible, the pulse rise-time 
was sacrificed. Normally rise-times of $\sim 10 \mu$ s were obtained leaving only the remaining $10 \mu \mathrm{s}$ as a flat top. This longer power supply was used in experiments aimed at determining the ion source capabilities at extended pulse lengths.

\subsection{Mechanical Design of the Ion Gun}

Since the main objective of this effort has been to explore the high current density regimes of surface ionization - type sources, it was required to provide heating power sufficient to raise the surface temperature of the emitter beyond the typical $\sim 1,000{ }^{\circ} \mathrm{C}$ operating temperature of lower current density yielding designs.

In order to accomplish this purpose, a multiple filament heating module was designed. Four filaments surround a molybdenum alloy emitter holder that allows for an easy interchange of the cylindrical emitters. The filaments themselves are provided with mechanical strength through molybdenum-rhenium tubes that are brazed along the filament leads. Several centimeters away from the heating module, these Mo-Rh leads are held by copper pieces that also provide the electrical contact for powering the heater. This design allows for a filament circuit that is electrically insulated from the structural components of the module and the heat shields, at the same time that avoids the use of ceramic insulators. With this design, since the filaments are allowed to move and expand freely without any resistance, then the risk of breaking the filaments during operation is greatly reduced.

The main heater cage components are entirely made out of refractory metals to sustain the temperatures required. Molybdenum-rhenium and pure molybdenum were used in the heat shields. Tungsten was used in the source holder and main supporting 
plate. Figure 3.2 shows a photograph of the heater main cage and Figure 3.3 a photograph of the actual module on its supporting structure.

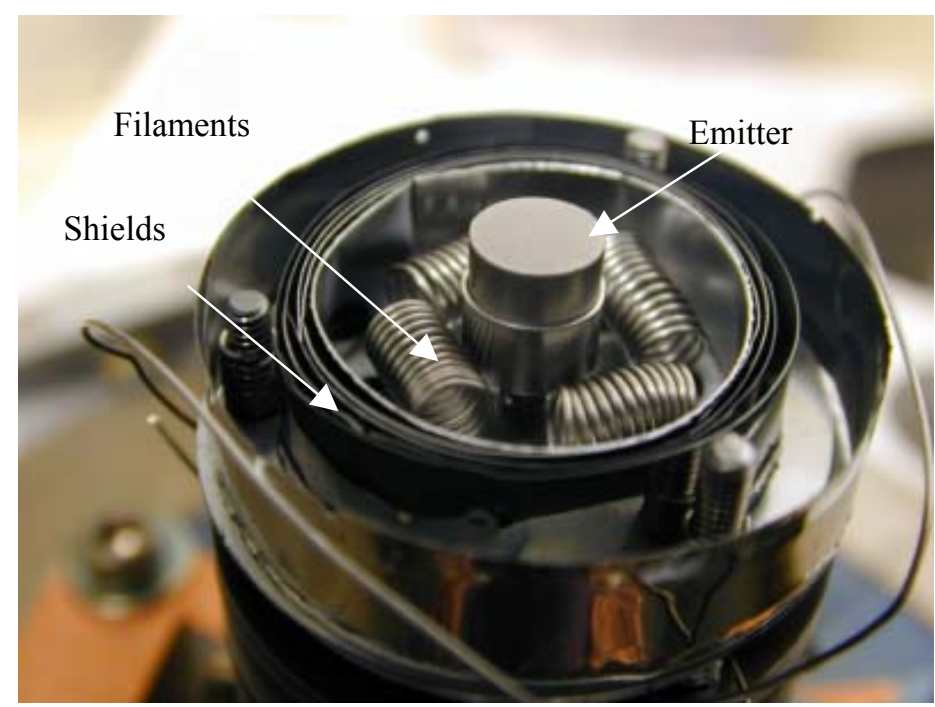

Figure 3.2. Main heater cage showing filaments and main source emitter.

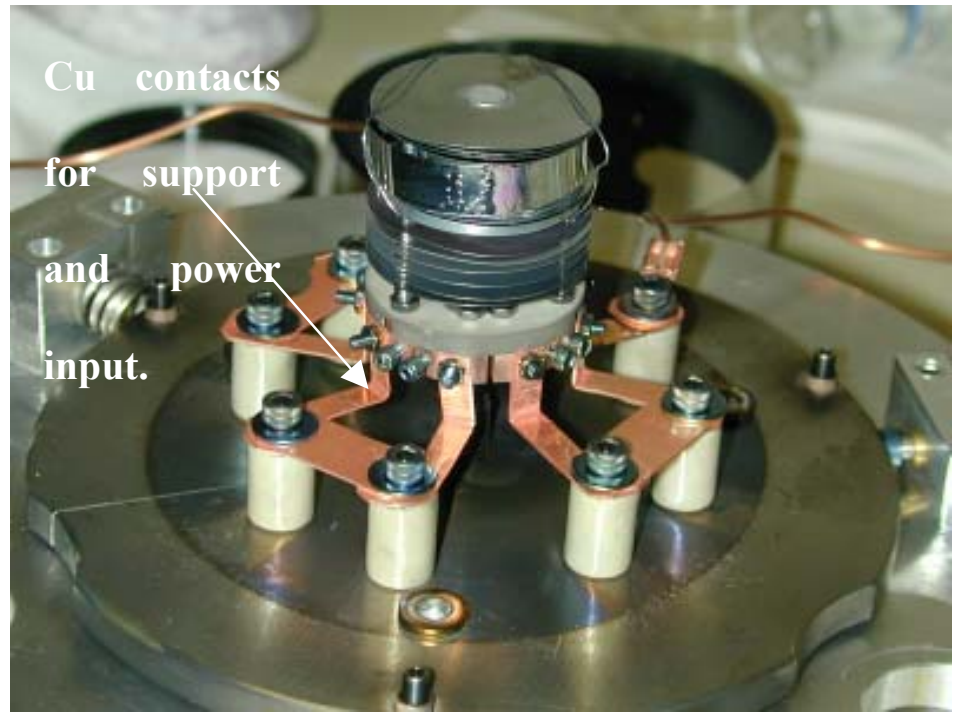

Figure 3.3. Heater module.

The heater module is supported by ceramic stands that in turn provide the required electrical insulation for the copper leads that power the source. 
Figure 3.4 shows the typical dependence of the source temperature with respect to the electrical power input. These curves varied to some extent from source to source given the small variations in the position of the shields and the source type. There is plenty of room for improvement in this design in terms of thermal efficiency. The corresponding efficiencies at $765^{\circ} \mathrm{C}$ and $1,130{ }^{\circ} \mathrm{C}$, calculated by dividing the radiant power at the emitter surface by the input power are $3.8 \%$ and $4.2 \%$ respectively.

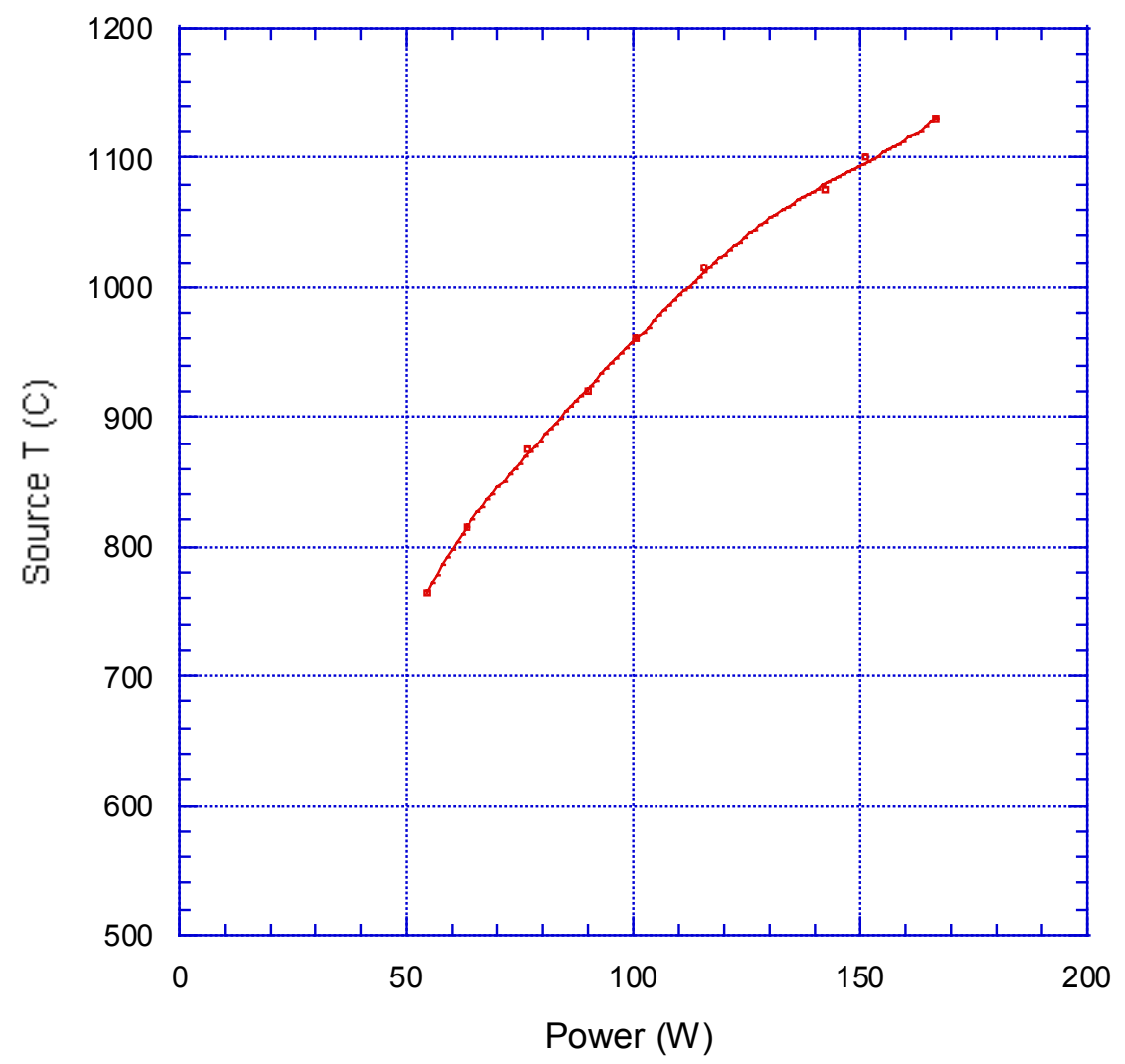

Figure 3.4. Typical dependence of the source temperature with respect to the electrical power input.

The plate on which the module is attached, as seen in Figure 3.3, is in contact with the main supporting structure through adjustment screws that may provide movement in the vertical and horizontal directions. This is a convenient way to align the 
center of the emitter with respect to the extraction plate, as well as provide a means for variable perveance if the vertical height of the source is changed. The assembled ion source can be seen in Figure 3.5.

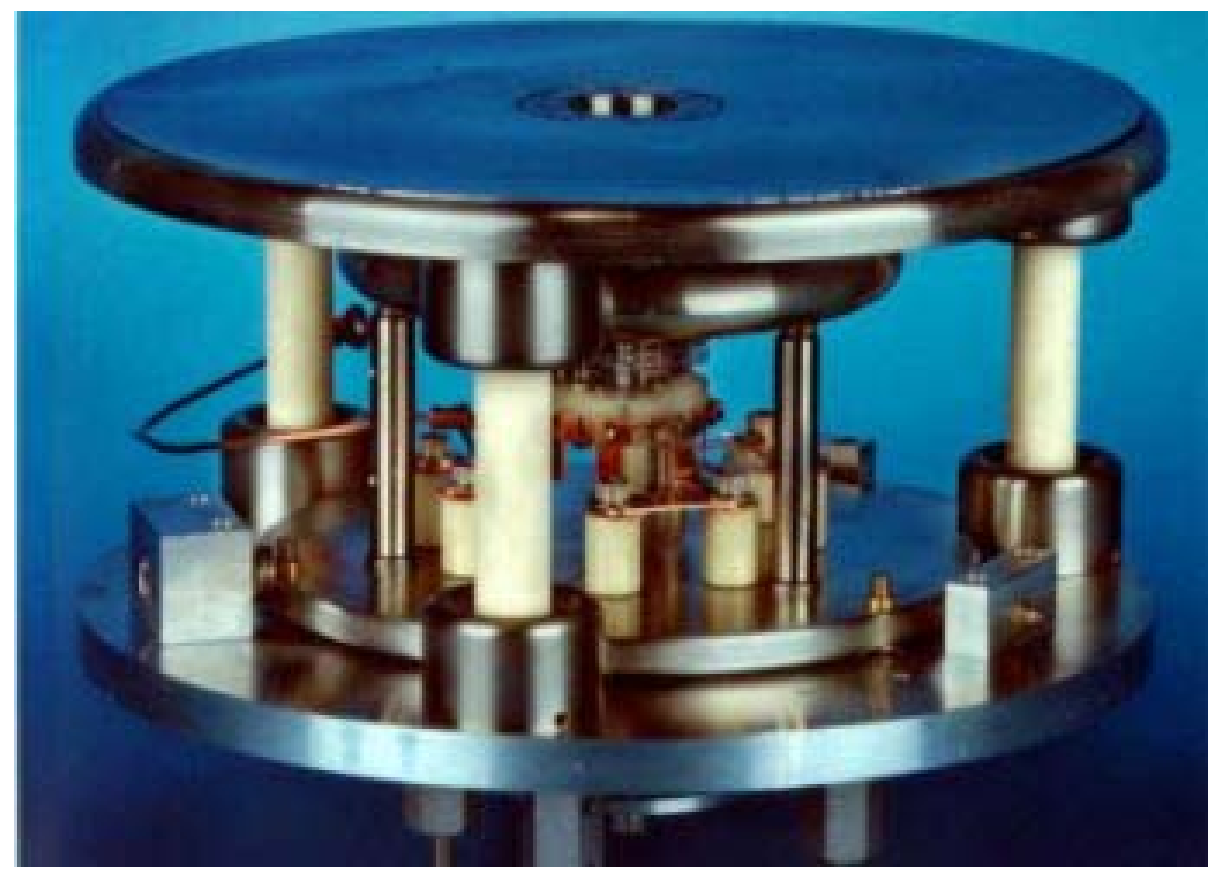

Figure 3.5. High J ion gun with extraction plate on.

The multiple heater approach is not based on efficiency considerations but rather on the need to guarantee high temperatures at the emitter surface. Nevertheless, the multiple heater design would be applicable to a multiple beamlet system. Through the use of multiple filaments, an array of sources can be made in which the efficiency would increase as more sources are included, thanks to the reduction of heat loss area and presumably a lower operating temperature required on the filaments. 


\subsection{Diagnostics}

The main diagnostics used in these experiments were a large faraday cup, a neutral particle detector and a double slit scanning system. These instruments are described in the following section.

\subsubsection{Faraday Cup}

Since the main figure of merit to assess the ion yield from a source is the average current density emitted, the main diagnostic used in these experiments has been a Faraday Cup (FC). The FC used consists of a grounded cylindrical casing that encloses two electrodes, electrically insulated from the casing. Figure 3.6 and 3.7 show the relevant features of this diagnostic.

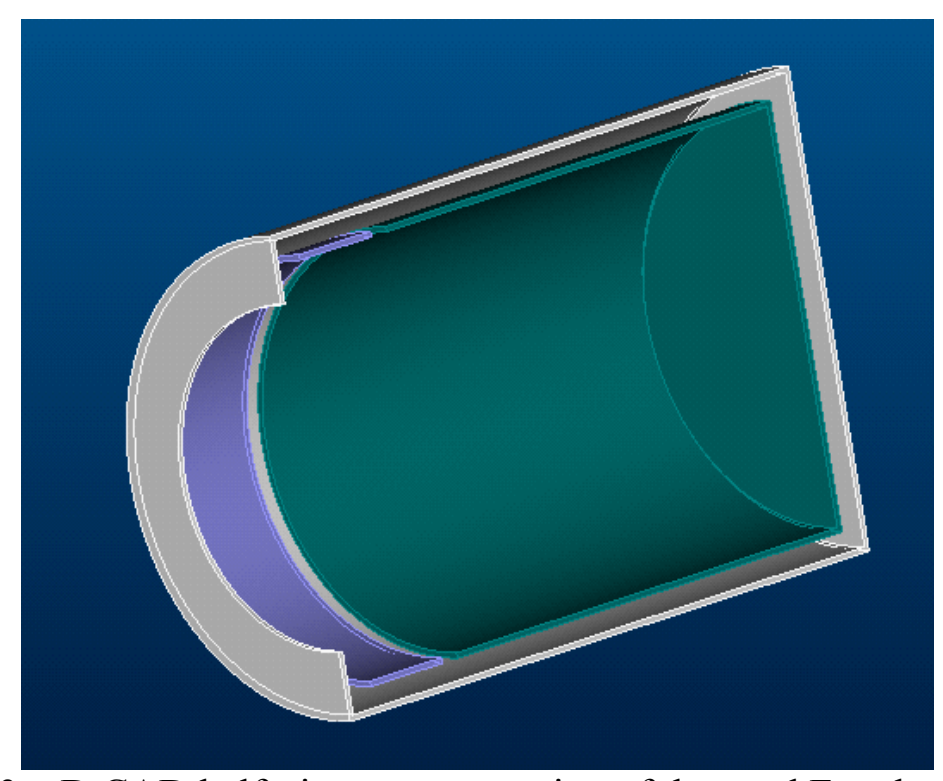

Figure 3.6. 3-D CAD half-view representation of the used Faraday Cup. 


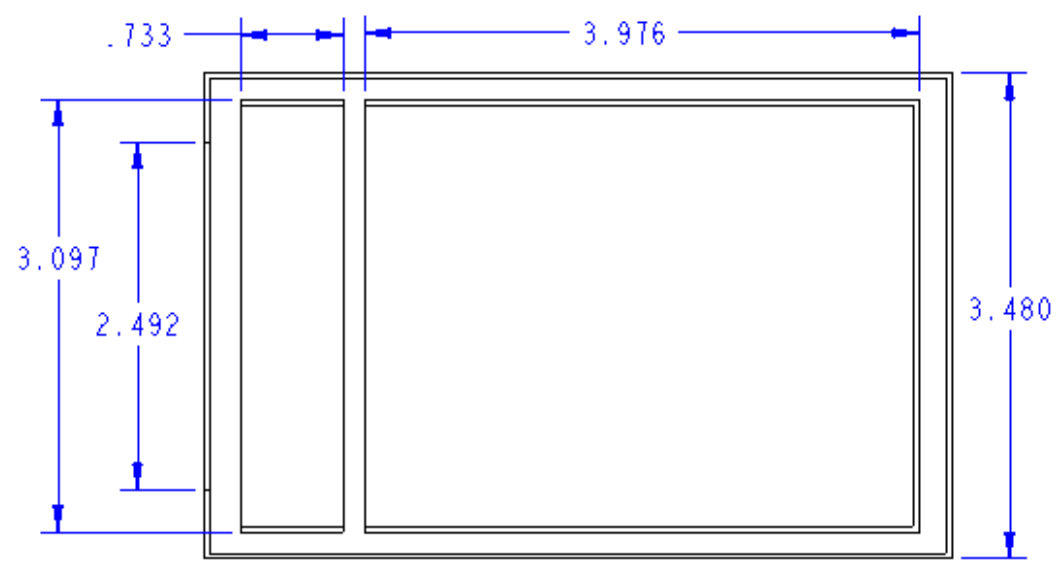

Figure 3.7. Faraday Cup dimensions, in inches.

The larger electrode, the collector, is shaped to receive the incoming ion beam. The excess charge on the collector has a path to ground through a coupling circuit that allows the application of a biasing voltage as well as a monitoring of the arriving current. A schematic of the coupling circuit used is shown in Figure 3.8. The collector is usually biased positive, to attract the secondary electrons that are generated when the ion beam strikes. A honeycomb shaped structure made with sheet metal is inserted at the end of the collector. This aids in shaping the fields and giving depth to the potential region with positive value, further helping in electron confinement.

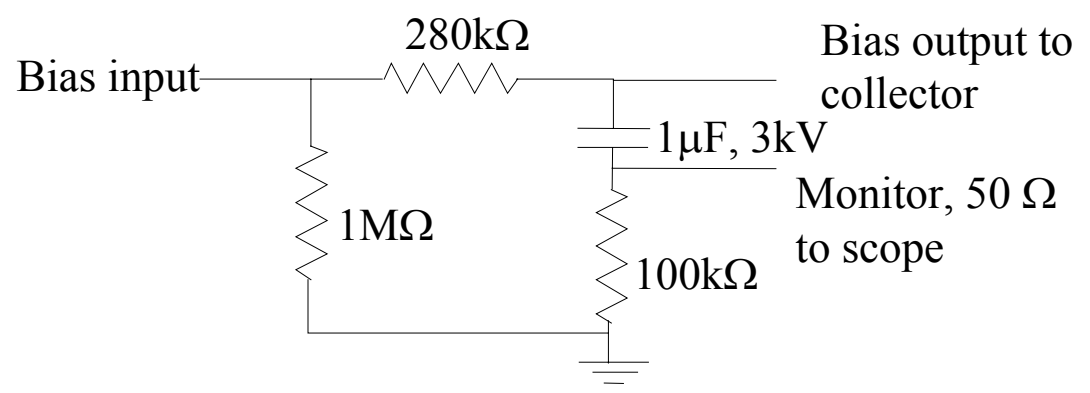

Figure 3.8 Coupling circuit diagram. 
The second electrode, the suppressor, is a ring at the entrance of the collector. The purpose of the suppressor is to establish a region of negative potential of such magnitude that secondary electrons generated at the collector can be forced back into the collector region. The suppressor voltage also helps to reject incoming low energy electrons that may travel with the ion beam.

\subsubsection{Neutral Particle Detector}

Neutral particle emission is of paramount importance in the studies of contact ionization sources. At any given moment there is a fraction of the total population of alkali atoms that exists in neutral form on the metallic substrate. Their continual evaporation constitutes a loss mechanism for the alkali atoms and may further have other unwanted consequences. For instance, an ion beam could degrade due to charge exchange processes as it moves through a neutral background, and the continual deposition of alkali atoms on insulator surfaces could pose a threat to their voltage holding capabilities. Neutral particle emission theory from tungsten surfaces has been elaborated elsewhere (Langmuir \& Taylor, 1933); however, a need for direct measurement of this process has existed in HIF research given the diversity of non-ideal conditions of the sources under consideration (diverse degrees of surface porosity, contaminants, unknown alkali surface coverages, etc.)

The essential features of this device are shown in Figure 3.9. The neutral detector operation is based on the principle of ionizing the neutral efflux emitted by a source, with the subsequent measurement of the current generated (in analogous way to how a vacuum ion gauge works). This is accomplished by means of a thin platinum foil that is heated to 
an operating temperature of about $1,050{ }^{\circ} \mathrm{C}$ by a DC current driven through it. The $\mathrm{Pt}$ foil is exposed to the ion source. Neutral particles travel into the detector and impinge the platinum foil that in turn will ionize the arriving neutrals with high efficiency due to its high electron work function of $5.39 \mathrm{eV}$. A negatively biased collector applies a constant electric field on the platinum foil, extracting the newly formed ions and collecting them. The casing of this setup has been biased positively to prevent the ions from striking the casing or leaving the detector. Grids of $90 \%$ transparency are located in the casing and collector apertures to ease the collection of particles, aid in shielding and improve the field uniformity.

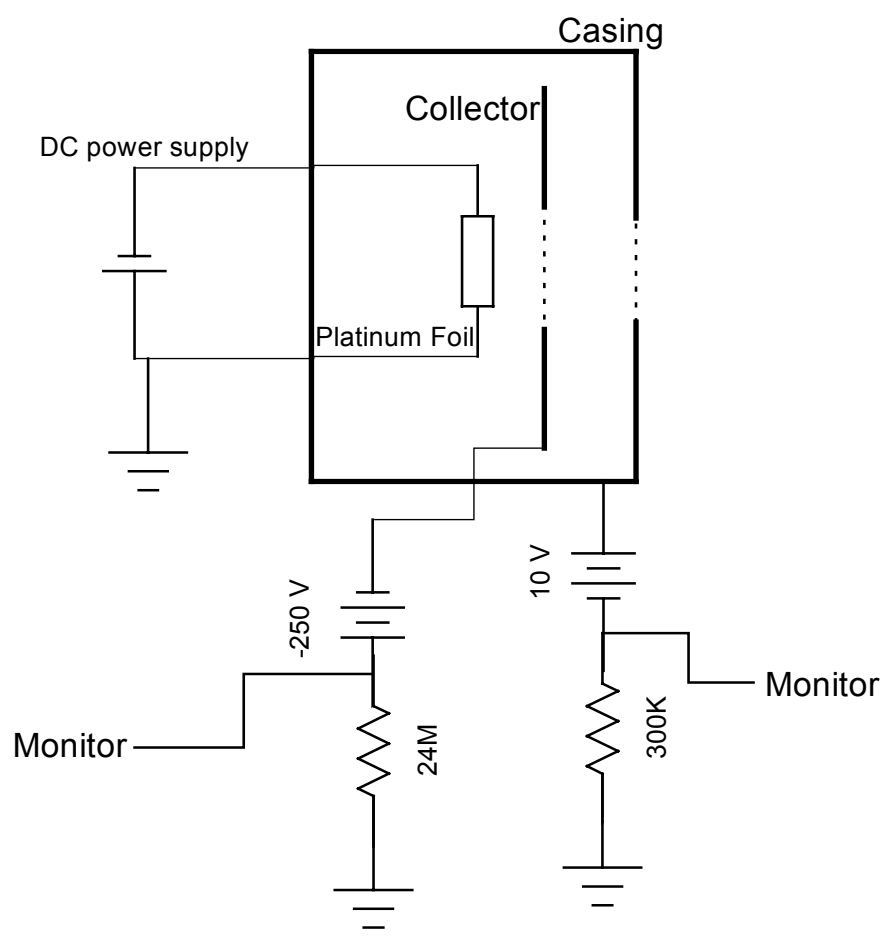

Figure 3.9 Schematic of the neutral particle detector.

Ions arriving at the collector make a very small current that is measured through the voltage drop across a large resistance on its path to ground. A special multimeter 
with $10 \mathrm{G} \Omega$ input impedance was used, given the large value of the resistor chosen. Another voltage monitor was put to inspect for any currents arriving or leaving the casing and that would be identified as sources of error. No such currents were identified.

The conversion factor that translates currents measured at the neutral detector to neutral particle density emitted at the source is purely determined by the setup geometry and is estimated in direct analogy with the shape factors common in radiation heat transfer. In this way, the total current arriving at an area $A_{2}$ due to a particle efflux emanating from an area A1 is given by the relation:

$$
I_{A 2}=J_{0}^{0} A_{1} F_{1-2}
$$

where $\mathrm{J}_{0}{ }^{0}$ is the neutral particle efflux density at the source, and the term $\mathrm{A}_{1} \mathrm{~F}_{1-2}$ is given by the relation:

$$
A_{1} F_{1-2}=\int_{A_{1}} \int_{A_{2}} \frac{\cos \left(\theta_{1}\right) \cos \left(\theta_{2}\right)}{\pi r^{2}} d A_{2} d A_{1}
$$

here ' $r$ ' is the distance between two area elements $\mathrm{dA}_{1}$ and $\mathrm{dA}_{2}$, and $\theta_{1}$ represents the angle with respect to the normal at which $\mathrm{dA}_{2}$ is "viewed" from the center of $\mathrm{dA}_{1}$. The angle $\theta_{2}$ is interpreted in an analogous way but with $\mathrm{dA}_{2}$ as the reference. In our particular case, the rectangular platinum foil $\left(\mathrm{A}_{2}\right)$ and the circular source $\left(\mathrm{A}_{1}\right)$ are parallel and $\theta_{1}$ and $\theta_{2}$ are equal. Equation (3.2) also includes the assumption of emission following Lambert's cosine law for the particle emission. Typical values of $\mathrm{A}_{1} \mathrm{~F}_{1-2}$ of $2 * 10^{-3} \mathrm{~cm}^{2}$ were estimated at the detector - source distances used. Figure 3.10 shows a convenient visualization of the shape factor dependence with the operating distance. 


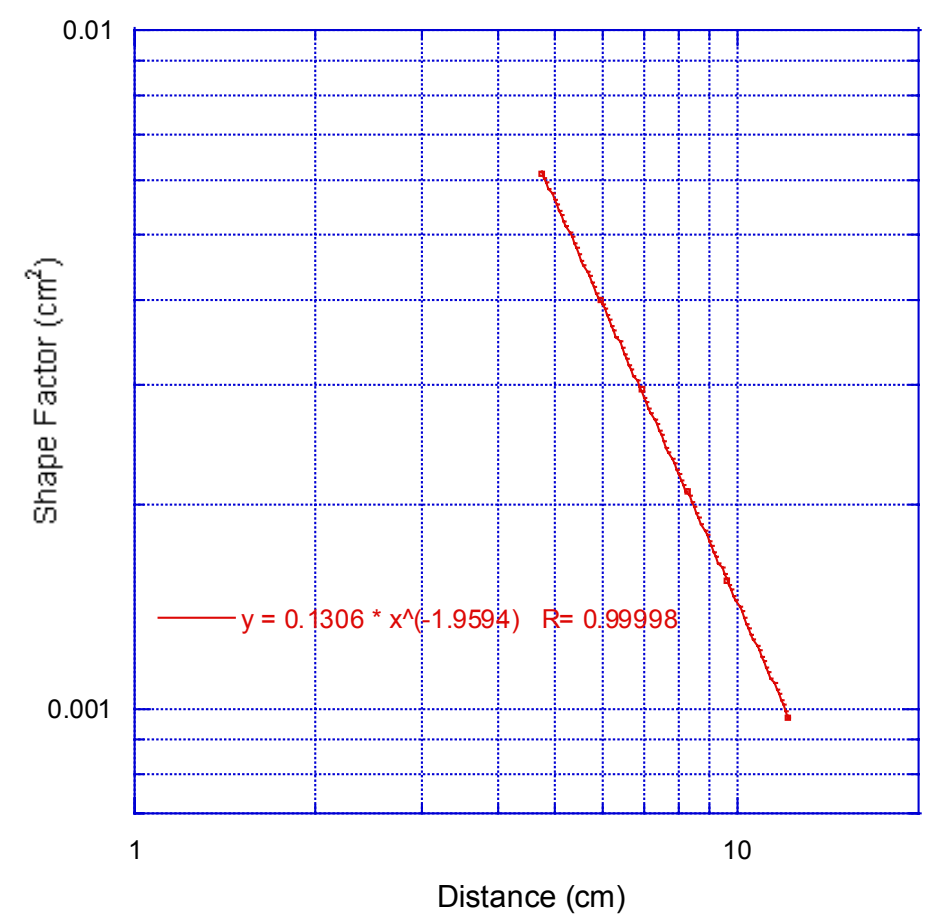

Figure 3.10. Dependence of the shape factor between the $1 / 4$ " source and the Pt foil in the neutral detector on the distance between them.

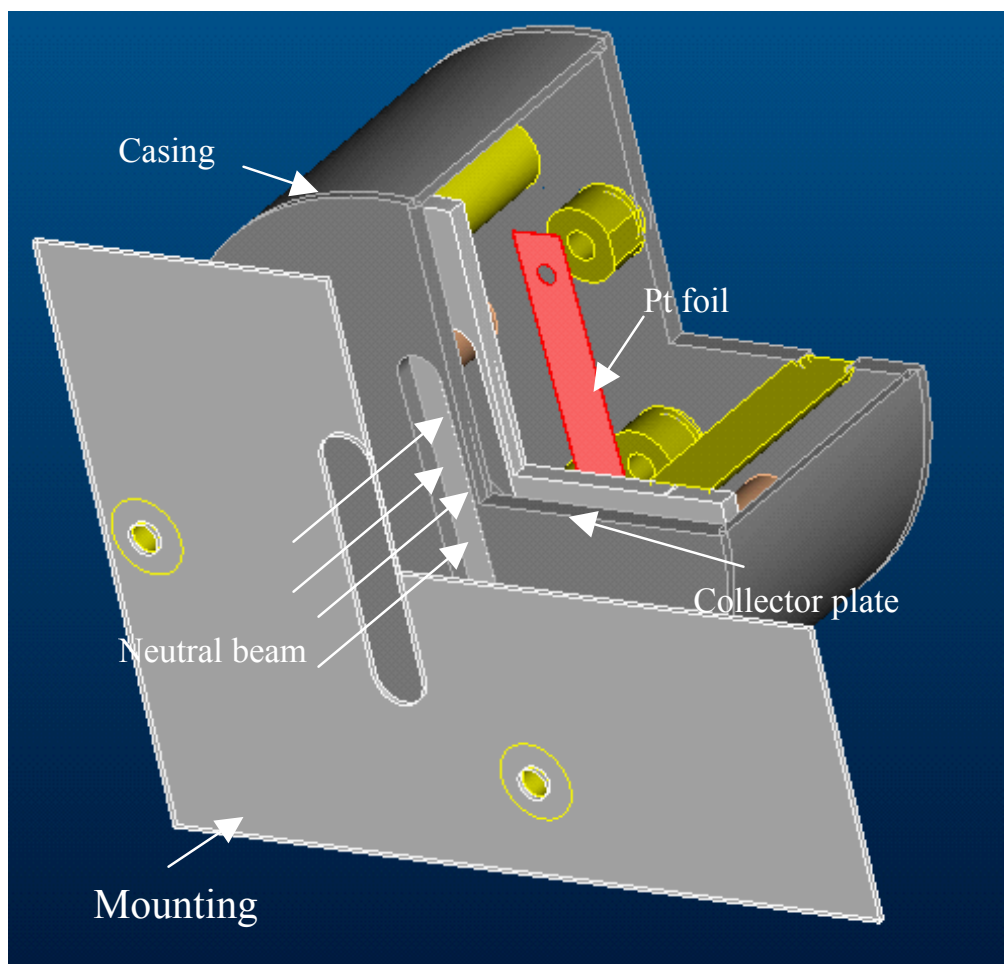

Figure 3.11. CAD representation of the neutral detector. 


\subsubsection{Double slit scanner.}

The double slit scanner is a standard diagnostic used throughout the HIF experiments. The system consists of two movable thin slits that move across the beam perpendicular to its direction of propagation. They serve a dual purpose: first, if only one slit is used, by detecting the signal of a beam that gets through this slit, the transverse intensity profile of the particle beam can be resolved. The single slit measurement effectively integrates over the direction parallel to the slit's length, so the scanning of this uniform (and axisymmetric) beam would in principle yield an inverted parabola as a profile. Comparison of the measured beam with respect to this uniform ideal is done this way. The second purpose involves the dual action of both slits. When the beam strikes the first slit, a "sheet beam" will emerge downstream. The natural expansion of the envelope of this sheet beam will occur due to two reasons. On one hand, the self-fields of the beam will push the outer particles outward, hence increasing the size of the beam. On the other, even if the beam were neutral, the random motion of the particles within the beam would also induce an increase of the dimensions of the sheet beam. The setup geometry is chosen such that the later effect is dominant. The second slit can thus be moved across the sheet beam to sample this expansion. The expansion or divergence of this sheet beam is intimately related with a fundamental beam property, the emittance, as will be seen next. Figure 3.12 shows a cartoon representing the action of the two slits in the emittance measurement. 


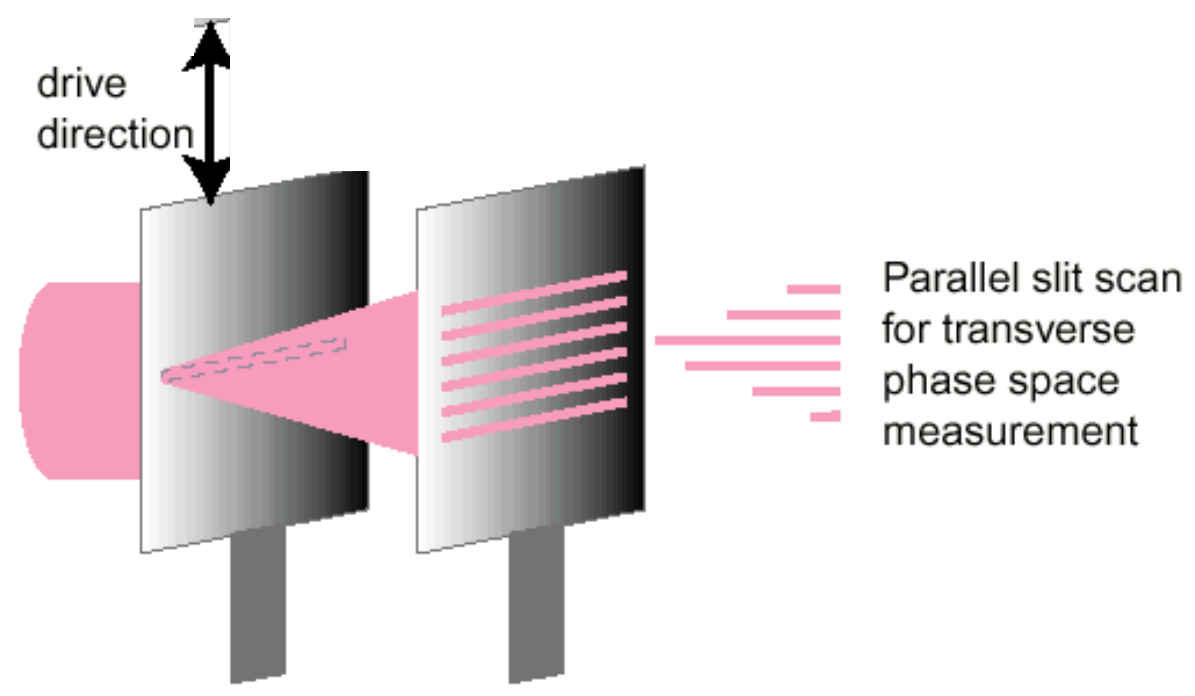

Figure 3.12 Schematic of the action of the double slit system for measuring the emittance. (Taken from MacLaren, 2000.)

It is useful at this point to go through derivation of the relevant quantities that can be measured with this setup, starting with the envelope equation. Consider the equation that represents the envelope of a particle beam of elliptical section as a function of the propagation distance " $z$ ":

$$
a^{\prime \prime}=k_{x}(z) a+\frac{2 Q}{a+b}+\frac{\varepsilon_{x}^{2}}{a^{3}}
$$

Here "a" and "b" are the semi axes of the ellipsoidal cross section of the beam, Q is the dimensionless perveance defined as

$$
Q=\frac{e I}{2 \pi \varepsilon_{0} m(\beta \gamma c)^{3}}
$$

and the $\mathrm{k}_{\mathrm{x}}$ terms represent the applied fields, or quadrupole strengths (depending on the magnetic or electrostatic transport being used):

$$
k_{x}=\frac{q E^{\prime}}{2 m v^{2}}
$$




$$
k_{x}=\frac{q B^{\prime}}{\gamma m v}
$$

What is desired to know is the dependence of the emittance term, $\varepsilon_{\mathrm{x}}$, in terms of measurable parameters. We start by noticing that the value of $<\mathrm{x}^{2}>$ over a the beam cross section is (assuming an axisymmetric beam of radius "a"):

$$
\left\langle x^{2}\right\rangle=\frac{a^{2}}{4}
$$

The first two derivatives of this expression (with respect to the direction of propagation, and cast in a convenient form) are:

$$
\begin{gathered}
a^{\prime}=\frac{4\left\langle x \cdot x^{\prime}\right\rangle}{a} \\
a^{\prime \prime}=\frac{4}{a}\left(\left\langle\left(x^{\prime}\right)^{2}\right\rangle+\left\langle x \cdot x^{\prime \prime}\right\rangle\right)-\frac{16}{a^{3}}\left\langle x \cdot x^{\prime}\right\rangle^{2}
\end{gathered}
$$

At this point, an expression for $<\mathrm{xx}^{\prime}$ ' $>$ is needed. A usable expression for $\mathrm{x}$ " can be derived starting from the expressions of the fields acting on the beam and the equation of motion:

$$
\begin{gathered}
\vec{E}=\frac{\lambda}{\pi \varepsilon_{0}} \frac{1}{a+b}\left(\frac{x}{a} \hat{x}+\frac{y}{b} \hat{y}\right)+\vec{E}_{e x t} \\
\vec{B}=\frac{\mu_{0} \lambda}{\pi} v \frac{1}{a+b}\left(\frac{x}{a} \hat{y}-\frac{y}{b} \hat{x}\right)+\vec{B}_{e x t} \\
\gamma m \ddot{x}=e\left(E_{x}-v B_{y}\right)
\end{gathered}
$$

Combining the above equations, expressing the external fields in the form $\mathrm{k}_{\mathrm{x}}(\mathrm{z}) \mathrm{x}$ and turning the time derivative into a spatial one (assuming constant axial velocity) we arrive at: 


$$
x^{\prime \prime}=k_{x}(z) x+\frac{e I}{\pi \varepsilon_{0} m(\beta \gamma c)^{3}} \frac{x}{a(a+b)}
$$

Finally, this expression can be used to calculate $<$ xx" $>$ yielding:

$$
\left\langle x \cdot x^{\prime \prime}\right\rangle=k_{x}(z)\left\langle x^{2}\right\rangle+\frac{2 Q}{(a+b)} \frac{1}{a(a+b)}\left\langle x^{2}\right\rangle
$$

Using the derived expression for $<\mathrm{xx}^{\prime}>$ in

$$
a^{\prime \prime}=\frac{4}{a}\left(\left\langle\left(x^{\prime}\right)^{2}\right\rangle+\left\langle x \cdot x^{\prime \prime}\right\rangle\right)-\frac{16}{a^{3}}\left\langle x \cdot x^{\prime}\right\rangle^{2}
$$

and using the relation

$$
\left\langle x^{2}\right\rangle=\frac{a^{2}}{4}
$$

we arrive at

$$
a^{\prime \prime}=k_{x}(z) a+\frac{2 Q}{(a+b)}+\frac{16}{a^{3}}\left(\left\langle x^{2}\right\rangle\left\langle\left(x^{\prime}\right)^{2}\right\rangle-\left\langle x \cdot x^{\prime}\right\rangle^{2}\right)
$$

Comparing with the original envelope equation (3.3) we find that the edge emittance can be expressed as

$$
\varepsilon_{\text {xedge }}=4 \varepsilon_{r m s} \equiv \sqrt{\left\langle x^{2}\right\rangle\left\langle\left(x^{\prime}\right)^{2}\right\rangle-\left\langle x \cdot x^{\prime}\right\rangle^{2}}
$$

At each position and angle, a certain signal amplitude is measured. The data obtained is sufficient to estimate all the required average values of the relevant quantities and finally yielding a measurement of the beam emittance in accordance with the formulas (3.19) through (3.22) 


$$
\begin{gathered}
\langle x\rangle=\frac{\sum_{x^{\prime}} \sum_{x} x \cdot \text { amplitude }}{\sum_{x^{\prime}} \sum_{x} \text { amplitude }} \\
\left\langle x^{\prime}\right\rangle=\frac{\sum_{x^{\prime}} \sum_{x} x^{\prime} \cdot \text { amplitude }}{\sum_{x^{\prime}} \sum_{x} \text { amplitude }} \\
\left\langle(x-\langle x\rangle)^{2}\right\rangle=\frac{\sum_{x^{\prime}} \sum_{x}(x-\langle x\rangle)^{2} \cdot \text { amplitude }}{\sum_{x^{\prime}} \sum_{x} \text { amplitude }} \\
\left\langle\left(x^{\prime}-\left\langle x^{\prime}\right\rangle\right)^{2}\right\rangle=\frac{\sum_{x^{\prime}} \sum_{x}\left(x^{\prime}-\left\langle x^{\prime}\right\rangle\right)^{2} \cdot \text { amplitude }}{\sum_{x^{\prime}} \sum_{x} \text { amplitude }} \\
\left\langle(x-\langle x\rangle) \cdot\left(x^{\prime}-\left\langle x^{\prime}\right\rangle\right)\right\rangle=\frac{\sum_{x^{\prime}} \sum_{x}(x-\langle x\rangle) \cdot\left(x^{\prime}-\left\langle x^{\prime}\right\rangle\right) \cdot \text { amplitude }}{\sum_{x^{\prime}} \sum_{x} \text { amplitude }} \\
\varepsilon_{x, \text { rms }}=\sqrt{\left\langle(x-\langle x\rangle)^{2}\right\rangle \cdot\left\langle\left(x^{\prime}-\left\langle x^{\prime}\right\rangle\right)^{2}\right\rangle-\left\langle(x-\langle x\rangle) \cdot\left(x^{\prime}-\left\langle x^{\prime}\right\rangle\right)\right\rangle^{2}}
\end{gathered}
$$

Equation (3.24) is the final equation that is used for the estimates presented in the following chapter.

It is also important to mention here the particular considerations relevant to the assessment of the most dominant factors that contribute to the expansion of the sheet beam, namely, the random motion versus the space charge expansion mentioned before. Upon exiting the first slit, the emerging sheet beam will have a natural tendency to expand due to its self-fields and the thermal motion of the particles. A similar analysis can be made to this sheet beam in terms of finding its envelope equation. In this case, no external fields are considered. 


$$
a^{\prime \prime}=K_{s}+\frac{\varepsilon^{2}}{a^{3}}
$$

where the perveance of the sheet beam is

$$
K_{s}=\frac{e J_{s}}{2 \varepsilon_{0} m(\beta \gamma c)^{3}}
$$

Since $\mathrm{K}_{\mathrm{s}}$ does not depend on a, the envelope equation can be integrated:

$$
a(z)=\sqrt{a_{0}^{2}+2 a_{0} a_{0}{ }^{\prime}+\left(\varepsilon^{2} / a_{0}^{2}+a_{0}^{\prime 2}\right) z^{2}}+K_{s} z^{2} / 2
$$

where the first term on the right hand side of the equation is the thermal contribution to the beam expansion and the last term is the self-field contribution. We consider a sheet beam produced at the center of the incident beam, so a' $=0$. Further, since emittance scales linearly with phase space dimensions, the ratio of the sheet beam emittance to its width is equal to that of the full beam to its radius. So

$$
a(d)_{\text {thermal }}=\sqrt{\frac{s^{2}}{4}+\frac{\varepsilon_{\text {beam }} d^{2}}{r_{\text {beam }}^{2}}}
$$

where $\mathrm{s}$ is the slit width, $\mathrm{r}_{\text {beam }}$ is the beam radius and $\mathrm{d}$ is the distance between the slits. At the same time, the contribution to the envelope expansion due to the self-fields can be expressed in terms of known quantities as:

$$
a(d)_{\text {s.c. }}=Q \frac{s d^{2}}{2 r_{\text {beam }}^{2}}
$$

The ratio of the expressions (3.28) and (3.29) gives the relative importance of both terms with respect to one another. In the experiments that are discussed on these pages, care has been taken to make the thermal contribution the dominant term, as the emittance is what is desired to measure. 


\section{Chapter 4: Experimental Results}

This section contains the experimental results obtained throughout these experiments. Information on the ion source analysis and preparation, ion and neutral particle yields as well as other relevant results is presented for the surface ionization sources. Some of these results have been published in the article "Development of High Current Density Surface Ionization Sources for Heavy Ion Fusion Applications", see references. 


\subsection{Contact Ionization Source Preparation.}

\subsubsection{Doping Calculation.}

The estimate for the amount of alkali-carbonate to be used to "dope" the sources is done as follows. The porosity $(\mathrm{P})$ of the emitter is estimated by direct measurement of its weight (w) and its volume $\left(\mathrm{V}_{\text {pellet }}\right)$, as well as knowledge of its theoretical density $\left(\rho_{\text {th }}\right)$ :

$$
P=1-\frac{w}{V_{\text {pellet }} \cdot \rho_{\text {th }}}
$$

Typically, the sources that were used in these experiments had porosity values between 20 and $30 \%$. A rough estimate of the number of grains $\left(\mathrm{N}_{\mathrm{g}}\right)$ contained in the solid body of the source is of course given by:

$$
N_{g}=\frac{V_{\text {pellet }} \cdot(1-P)}{V_{\text {grain }}}
$$

where $\mathrm{V}_{\text {grain }}$ is the volume of a single tungsten grain, as estimated by assuming a spherical shape and a diameter equal to the powder nominal size (between 6 and $12 \mu \mathrm{m}$ ). The total internal area $\left(A_{\text {int }}\right)$ in the emitter is estimated finally as

$$
A_{\text {int }}=N_{g} \cdot A_{g} \cdot k
$$

where $A_{g}$ is the surface area per grain and " $k$ " is a factor smaller than 1 that attempts to reflect the fact that a fraction of the grains surface is not part of the internal area due to the interconnectivity of the grains. A general value for this factor of 0.5 was used throughout.

The alkali atoms are deposited in the emitter in the form of a solution of the alkali carbonate $\left(\mathrm{K}_{2} \mathrm{CO}_{3}\right.$ or $\left.\mathrm{Cs}_{2} \mathrm{CO}_{3}\right)$ in de-ionized water. The amount of carbonate $\left(\mathrm{M}_{\text {carb }}\right)$ used 
to dope a single emitter was estimated to provide enough alkali atoms to cover the internal surface of the emitter with a single layer. This amount is given by

$$
M_{c a r b}=\frac{\sigma_{0} A_{\mathrm{int}} M_{w c a r b}}{N_{A}}
$$

where $\sigma_{0}$ is the number of alkali atoms in a single monolayer over a tungsten surface $(\sim$ $\left.4.8 \cdot 10^{14} \mathrm{~cm}^{-2}\right), \mathrm{M}_{\mathrm{wcarb}}$ is the molecular weight of the carbonate used and $\mathrm{N}_{\mathrm{A}}$ is Avogadro's number. Since the carbonate should be deposited with a single drop of water (with an estimated volume of $1.5 \cdot 10^{-2} \mathrm{ml}$ ), the concentration of the doping solution is finally estimated to be $3.73 \mathrm{~mol} / \mathrm{l}$. This solution was used in the contact ionization source experiments described in what follows.

\subsection{High Current Density Yields of $\mathrm{K}^{+}$and $\mathrm{Cs}^{+}$Sources}

\subsubsection{Performance of High Current Density Potassium on Tungsten Contact Ionization Sources.}

A high current density $(\mathrm{J})$, single gap diode ion source was designed and built to extract as much as $100 \mathrm{~mA} / \mathrm{cm}^{2}$ of a $\mathrm{K}^{+}$beam (see construction details in Chapter 3 .). The target current density was not achieved in the real ion gun as the original design calculations did not include the relevant "Pierce electrode - emitter gap" effects. These have a significant effect on the source output (for a simulation on the effects of this gap see Chapter 5). Average values of current obtained differed from the design parameters by about $10 \%$. Two extraction voltage pulsers were used: $70 \mathrm{kV}$ max, $2 \mu$ s pulse length and $50 \mathrm{kV} \max , 10 \mu \mathrm{s}$. The main parameter measured was the average $\mathrm{J}(<\mathrm{J}>)$. Experimental $<\mathrm{J}>$ vs. voltage $(\mathrm{V})$ curves were obtained for sources operating at different temperatures, as well as $<\mathrm{J}>$ vs. time curves at a fixed temperature. 
The nature of the operation of contact ionization sources makes their current output vary as the alkali atom content of the source varies. This will be discussed in more detail on Chapter 5. The variability of the current yield becomes more apparent at the more demanding regimes of high current density where the relative variation of the alkali coverage on the surface is more appreciable than in regimes of lower current density. Figure 4.1 shows an example of the maximum performance observed for a potassium doped contact ionization source. As it will be observed later, the output may be different at similar operating temperatures.

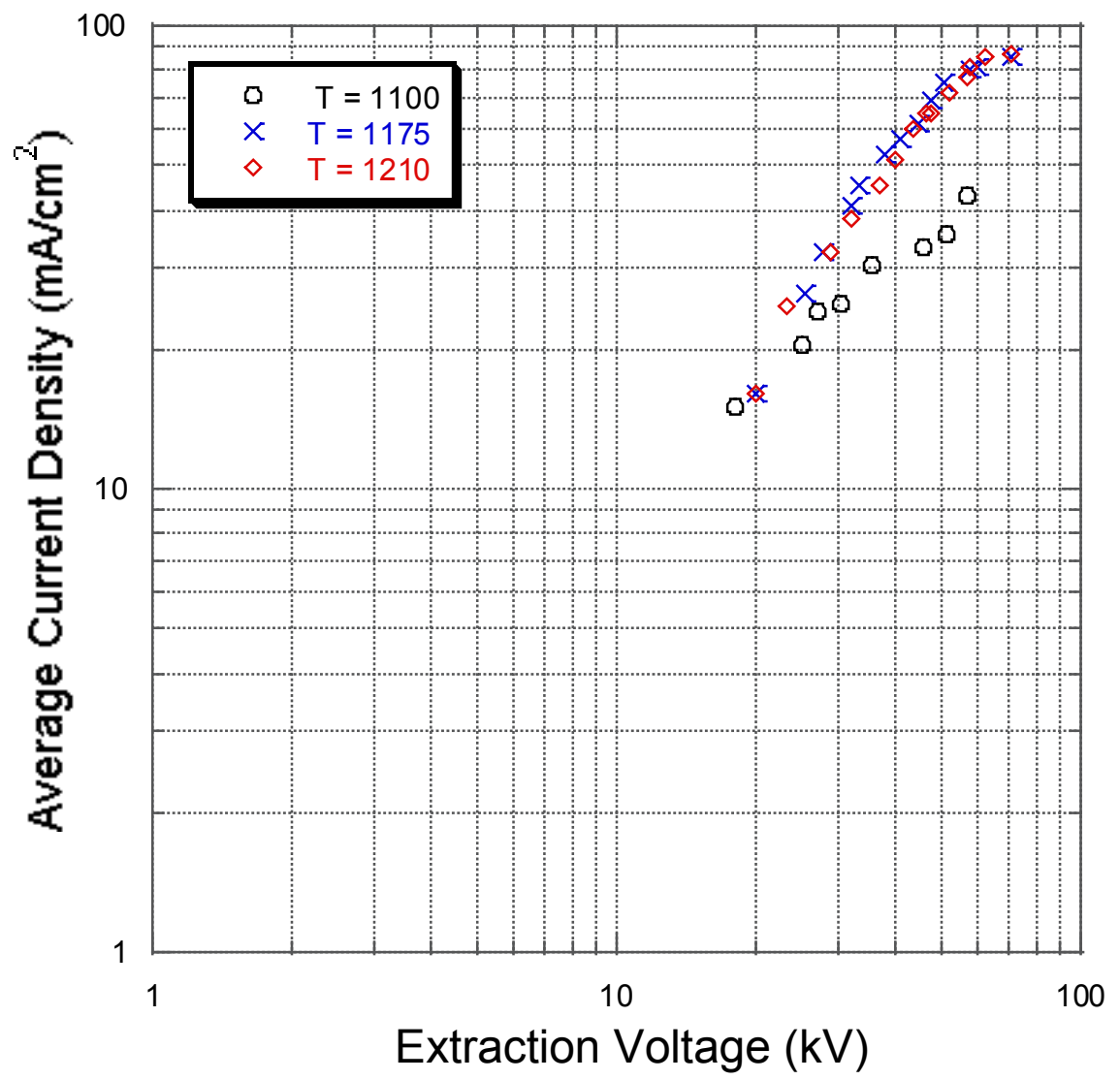

Figure 4.1 Maximum current density output performance observed for a potassium doped contact ionization source. 


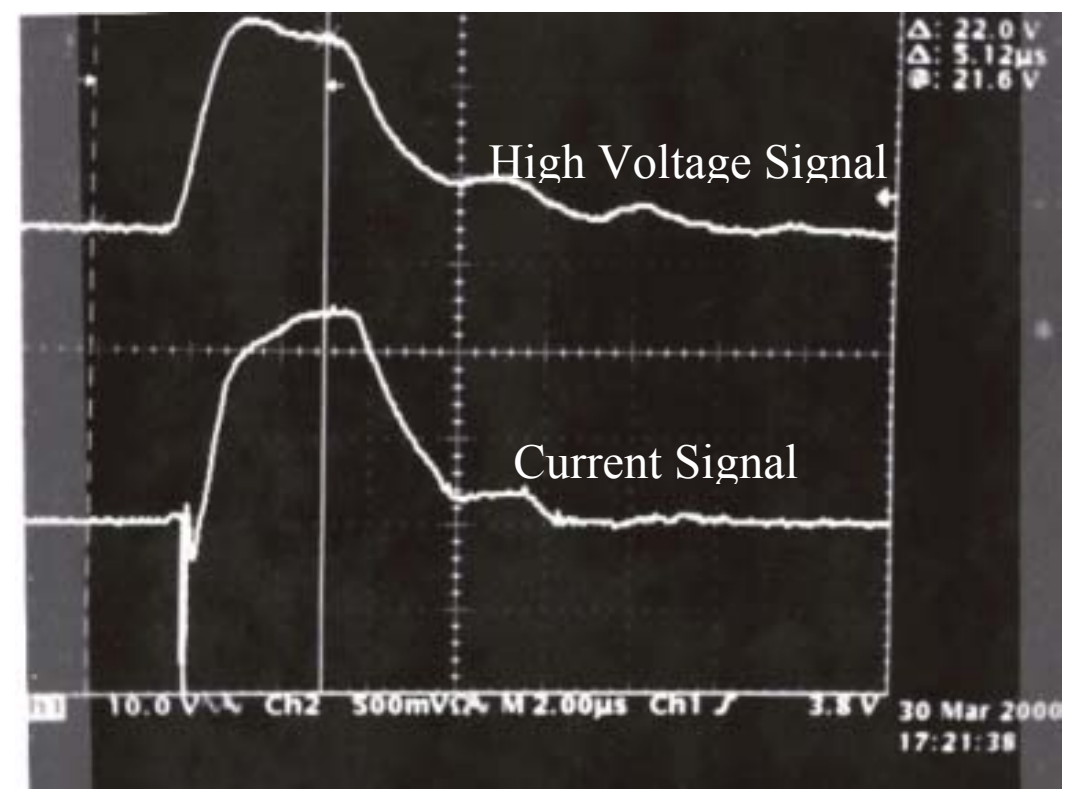

Figure 4.2 Scope trace showing the high voltage (positive pulse) and the current pulse .

The test described above was made with a "parallel plate" configuration, where the emitter surface was located in the same plane as the adjacent surfaces (in contrast with a Pierce electrode configuration), and parallel to the extractor plate. Further tests described below varied in their maximum output due to the incorporation of a Pierce electrode configuration, which despite its positive effect of providing some beam focusing, also concentrates the space charge in front of the emitter, decreasing the Child limit. This was a desirable incorporation during the early stages of the experiment in order to replicate conditions that would be present in a realistic, production ion source.

The variability of the current output with time can be seen in Figure 4.3, corresponding to the same emitter, but after 17 hours of continual operation at $1,145{ }^{\circ} \mathrm{C}$. It is clear that the maximum current output has decreased significantly. The maximum current density decreased to approximately $34 \%$ of its original value despite operating at a slightly higher temperature than before. 


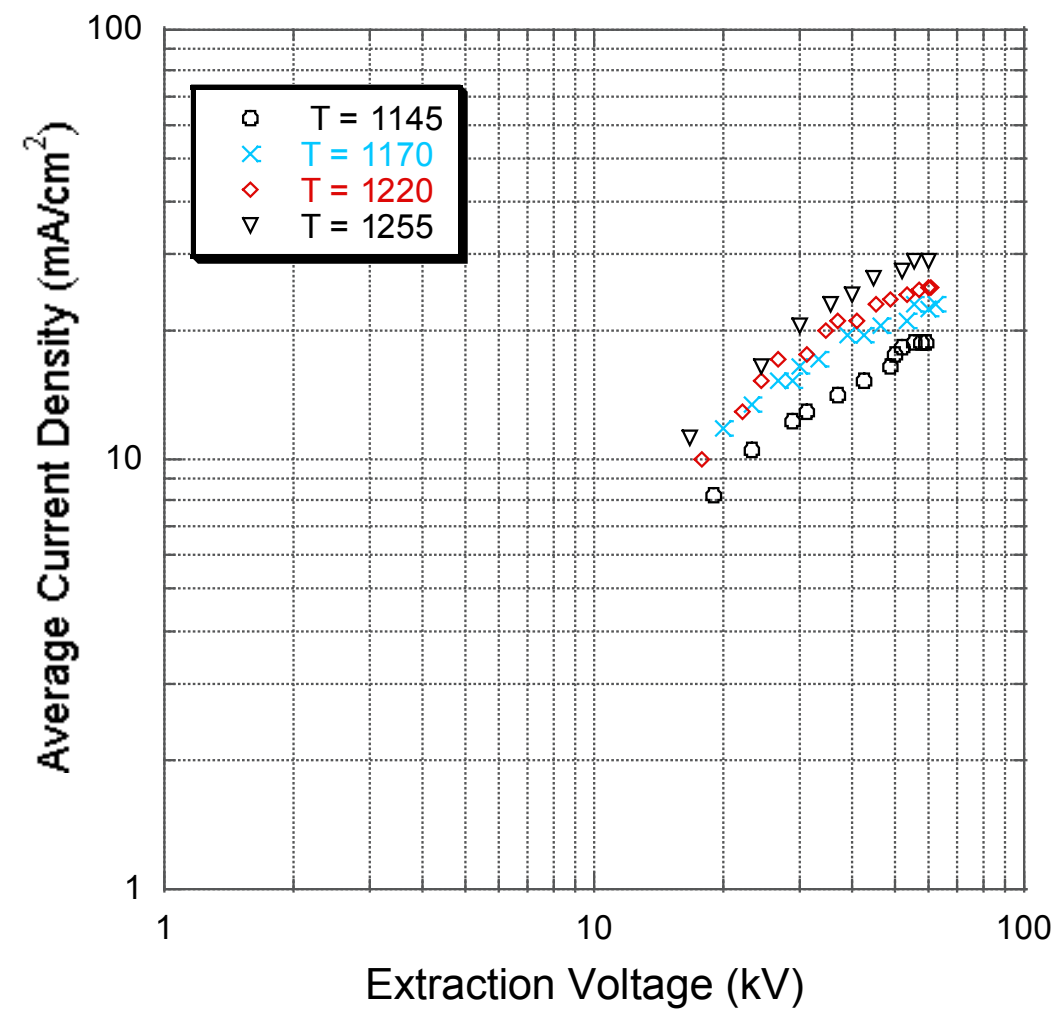

Figure 4.3 Decreased current output of a potassium doped tungsten source after 17 hours of continual operation at $1.145^{\circ} \mathrm{C}$.

Of interest is knowledge of the actual microscopic appearance of the emitter substrate used in the shots shown in Figures 4.2 and 4.3. The emitter for this test was made by pressing tungsten powder of a nominal size of $6 \mu \mathrm{m}$ in a 1/4" die with a $4,000 \mathrm{lb}$ force and subsequently sintering the pressed pellet at $1,950{ }^{\circ} \mathrm{C}$ for 3 hours in vacuum. Given the variations of the grain size with respect to the nominal size, the final features of the surface are not always uniform. Figures 4.4 and 4.5 show two different regions in the surface of the emitter near its central region. While Figure 4.4 displays an apparent uniformity of the porosity conditions in the emitter, it was found that the presence of regions where the number of pores per unit area was almost non-existent were common. Figure 4.5 shows an example of one of these regions. A combination of factors of sintering temperature, and tungsten grain size contribute to this phenomenon. 


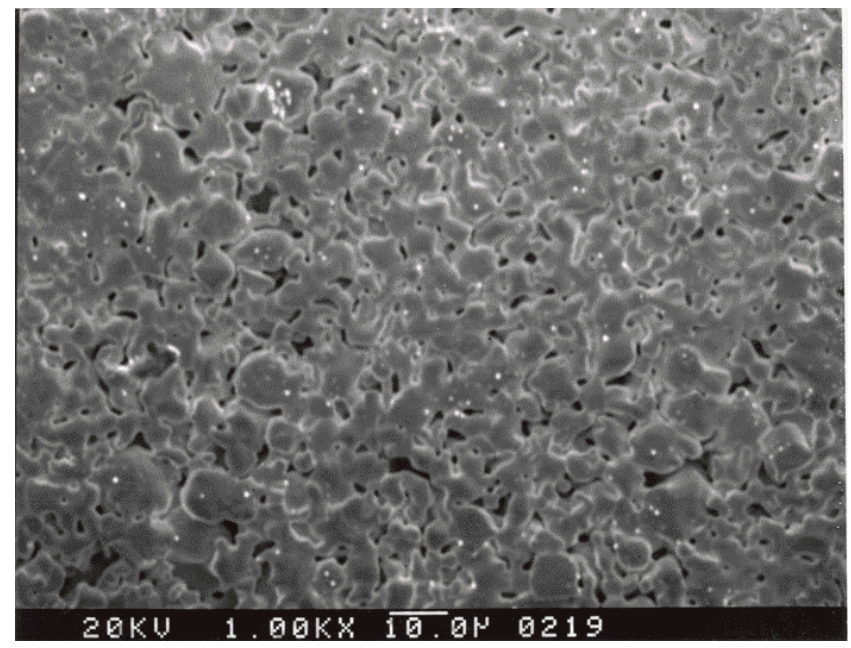

Figure 4.4. $6 \mu \mathrm{m}$ grain nominal size, sintered tungsten emitter, near center spot. Firing conditions: $1,950 \mathrm{C}, 3 \mathrm{~h}$ in vacuum.

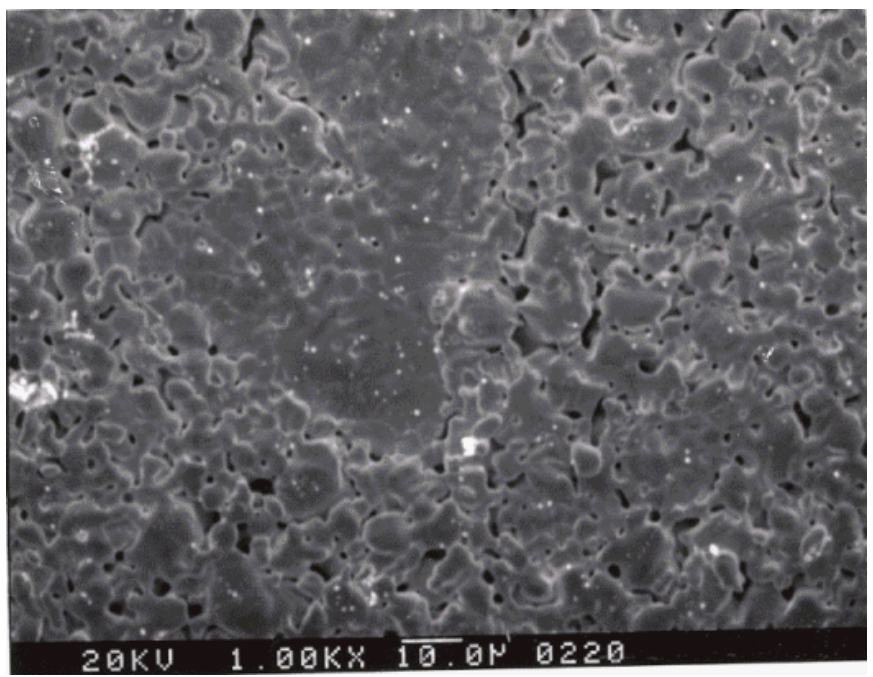

Figure 4.5. Same emitter as in Figure 4.46, different region near the center spot displaying one of the multiple regions of no porosity.

It was realized that the manufacturing conditions permitted the closing of pores in diverse regions throughout the emitter substrate. Regions with closed pores may give rise to spotty emission, as well as hinder the penetration of the dopant into the bulk material, decreasing the total content and lifetime. In a production source, where presumably there 
would be a back-feeding of the alkali atoms, this condition may prevent the desired uniform migration of the atoms towards the emitting surface.

The irregularities in surface porosity emerged also closer to the edge of the emitter. Figure 4.6 shows an SEM photograph of the edge of the same pellet. The difference in number of pores per unit area is evident. Edge-to-center variations of this sort are particularly important in emitters of small dimensions, since the relative contribution to the total current by the outer regions of the emitter is the largest.

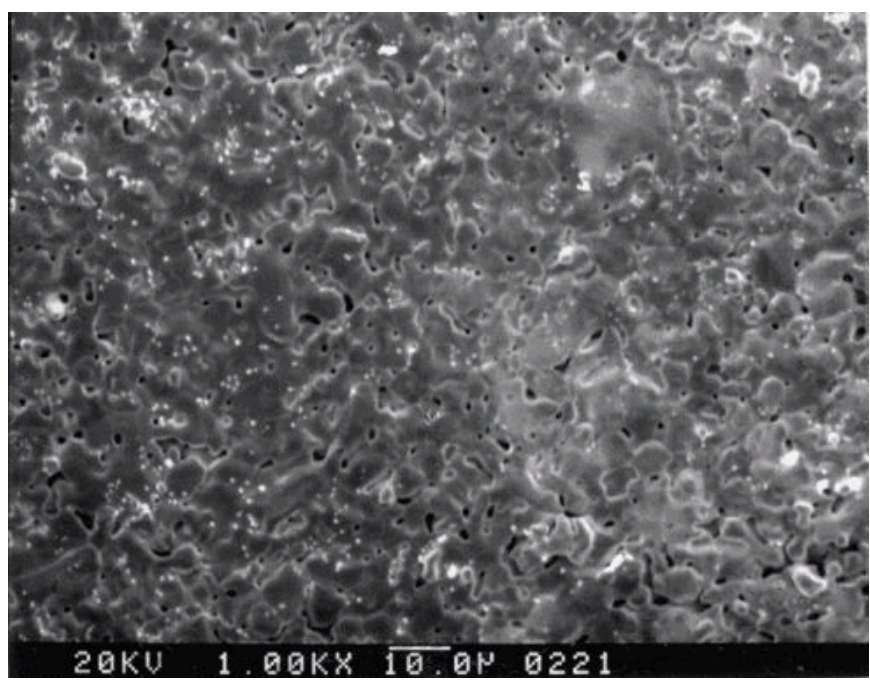

Figure 4.6. Same emitter substrate as shown in Figure 4.4. Region near the edge of the emitter. Note the differences in surface porosity with respect to the center of the emitter.

Other representative examples of observed performances in the high J regimes for potassium and cesium doped sources are presented next. The data sets that follow were intended to resemble each other in terms of the emitter preparation, alkali atom content and heating cycle. It is important to note that the decreased lifetime at the high current density regime, the current output and lifetime are dramatically affected by the (relatively short) time that the doped source spends at high temperatures, namely, temperatures in excess of $1,200{ }^{\circ} \mathrm{C}$. 
Figure 4.7 shows the average current density vs. extraction voltage curves measured for a potassium on porous tungsten contact ionization source. The ion gun space charge limit can be clearly appreciated and is represented by the straight $\mathrm{V}^{3 / 2}$ curve.

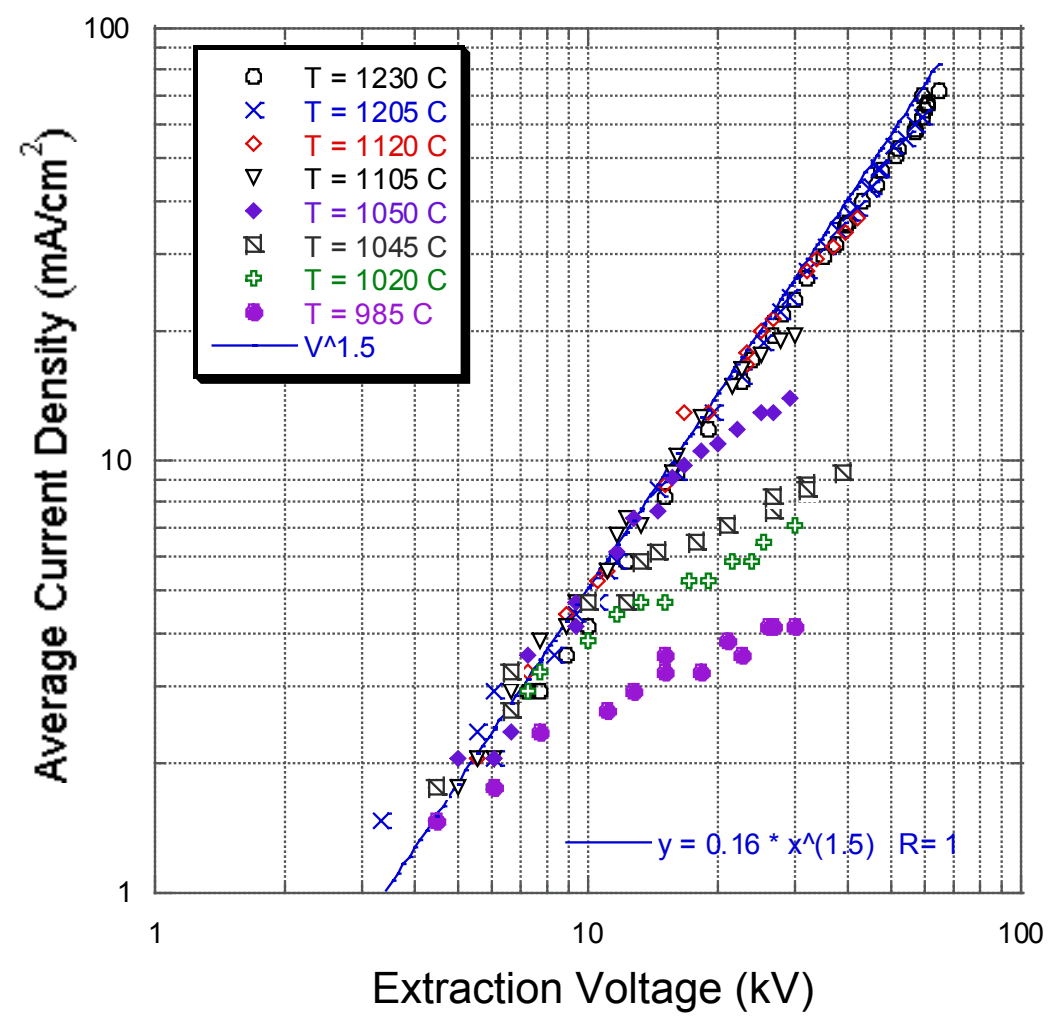

Figure 4.7. $\mathrm{J}-\mathrm{V}$ characteristics of a potassium on porous tungsten contact ionization source.

At temperatures above $1,120{ }^{\circ} \mathrm{C}$ it was not possible to see emission limited output (characterized by a deviation from the $\mathrm{V}^{3 / 2}$ behavior) at the tested extraction voltages. As it will be seen in the next graphs, the excursions to higher temperatures had a negative impact on the ion source lifetime despite the short duration of these excursions (typically less than 30 minutes).

During these tests, the ion source was run continually, no cooling down or reheating of the ion source was performed, with the sole exception of the high temperature 
excursions necessary to assess the source performance at different points during the operation cycle. The operating temperature for idling the ion source was chosen at a higher temperature than doped sources are usually operated in other HIF applications, usually around $1,100{ }^{\circ} \mathrm{C}$ in the present case. Figure 4.8 shows another family of $\mathrm{J}-\mathrm{V}$ characteristics for the same ion source after operating for 17 hours at $\mathrm{T}=1,075{ }^{\circ} \mathrm{C}$. At these conditions the source is unable to provide the initial performance, yielding a maximum of $60 \mathrm{~mA} / \mathrm{cm}^{2}$. In contrast to the recently doped source, temperatures above $1,200{ }^{\circ} \mathrm{C}$ were necessary to make the output insensitive to small variations in temperature. In other words, higher temperatures were needed to preserve a space charge limited emission throughout the source. The $\mathrm{J}-\mathrm{V}$ curve seems to maintain its position in the graph, in contrast to other tests carried out. In these, after a continued operation, small variations in the position of the emitter within the Pierce electrode had an appreciable impact on the measured output and the $\mathrm{J}-\mathrm{V}$ curves showed a downward displacement. 


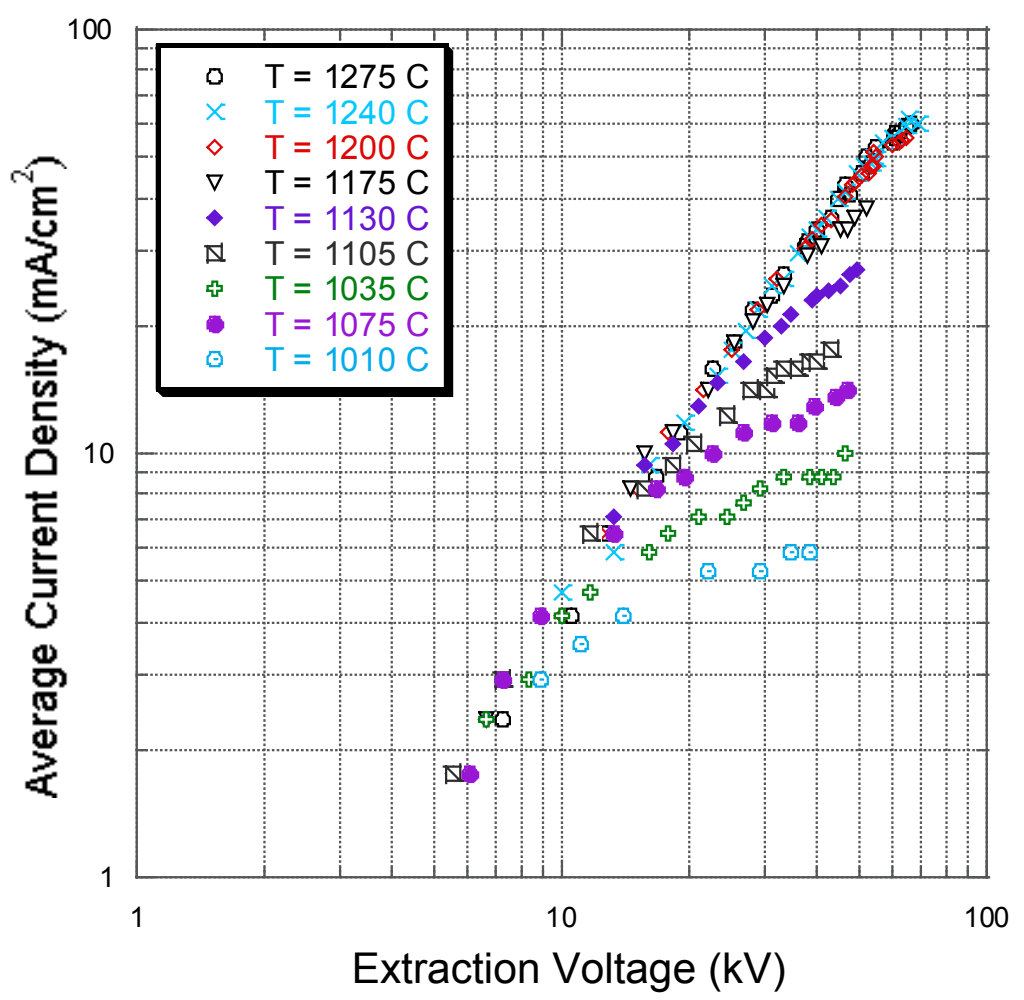

Figure $4.8 \mathrm{~J}-\mathrm{V}$ characteristics of a potassium doped tungsten source after almost $17 \mathrm{hr}$. of operation at $1,075^{\circ} \mathrm{C}$.

The final part of the heating cycle associated with this ion source consisted of a 33 hour period of operation at $1,100{ }^{\circ} \mathrm{C}$. The performance characteristics at the end of this period are depicted in Figure 4.9. The differences in ion output are evident when compared to the performance shown in the previous two figures. With the conditions shown in Figure 4.7 a space charge limited emission was obtained for the whole range of the applied voltages, for temperatures above $\sim 1,100{ }^{\circ} \mathrm{C}$. Under the conditions shown in Figure 4.8 there are deviations of up to $15 \%$ from the space-charge limit line at high current densities (above $60 \mathrm{~mA} / \mathrm{cm}^{2}$ ) at any temperature. In Figure 4.9 it can be seen that the maximum space-charge current density is below $10 \mathrm{~mA} / \mathrm{cm} 2$ at any temperature, including the maximum achievable of $1,300{ }^{\circ} \mathrm{C}$. The maximum emission limited current 
density achieved under these conditions is less than $30 \%$ of the maximum space charged current shown in Figure 4.7.

In the conditions shown in Figure 4.9 the performance of such ion source only matches the requirements of large HIF ion sources operating at a few $\mathrm{mA} / \mathrm{cm}^{2}$. For a large emitter required to deliver an average current density of around $4 \mathrm{~mA} / \mathrm{cm}^{2}$, there would have been no perceived change in the performance of the source despite the temperature excursions that the source has been subject to. The rapidly changing character of the alkali atom content can be appreciated when noting that the ion yield was actually decreasing during the measurement of the characteristics at temperatures above $1,250^{\circ} \mathrm{C}$. This is evidenced in Figure 4.10.

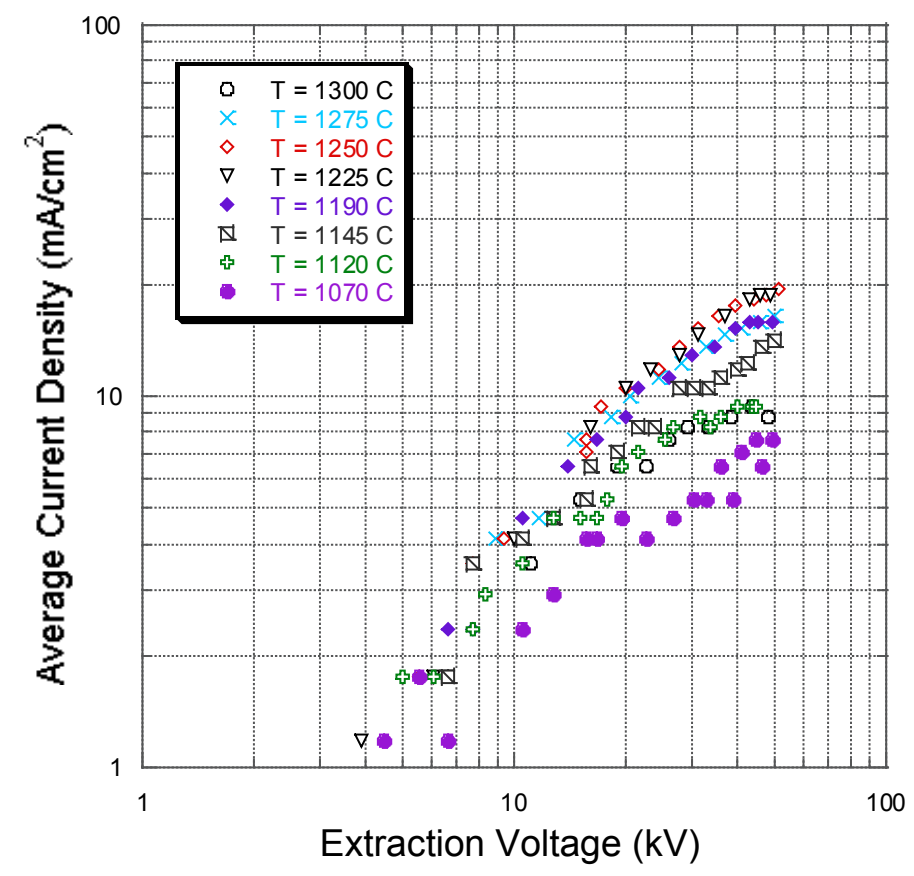

Figure 4.9. $\mathrm{J}-\mathrm{V}$ characteristics of a potassium doped tungsten source after $50 \mathrm{hr}$. of operation. Last 33 hours at $1,100^{\circ} \mathrm{C}$. 
Figure 4.10 shows the current output at a fixed voltage measured while the source operated at $1,300{ }^{\circ} \mathrm{C}$, very close to the maximum temperature attainable by the ion gun heater. The changes in ion current at that temperature and level of alkali atom content occur in a time scale of minutes as shown in the graph. At higher operating temperatures, various effects contribute to a decreased lifetime:

1. The ratio of the number of neutral atoms to ions throughout the emitter increases with temperature by virtue of the equilibrium condition required by the Saha equation (Equation 5.1).

2. The rate at which the neutral atoms are evaporated increases with temperature. Neutrals migrate faster through the emitter substrate and are evaporated faster once they are at the surface since their mean lifetime on the surface is reduced (see discussion in Chapter 5).

3. The amount of ions that are extracted during the pulse increases.

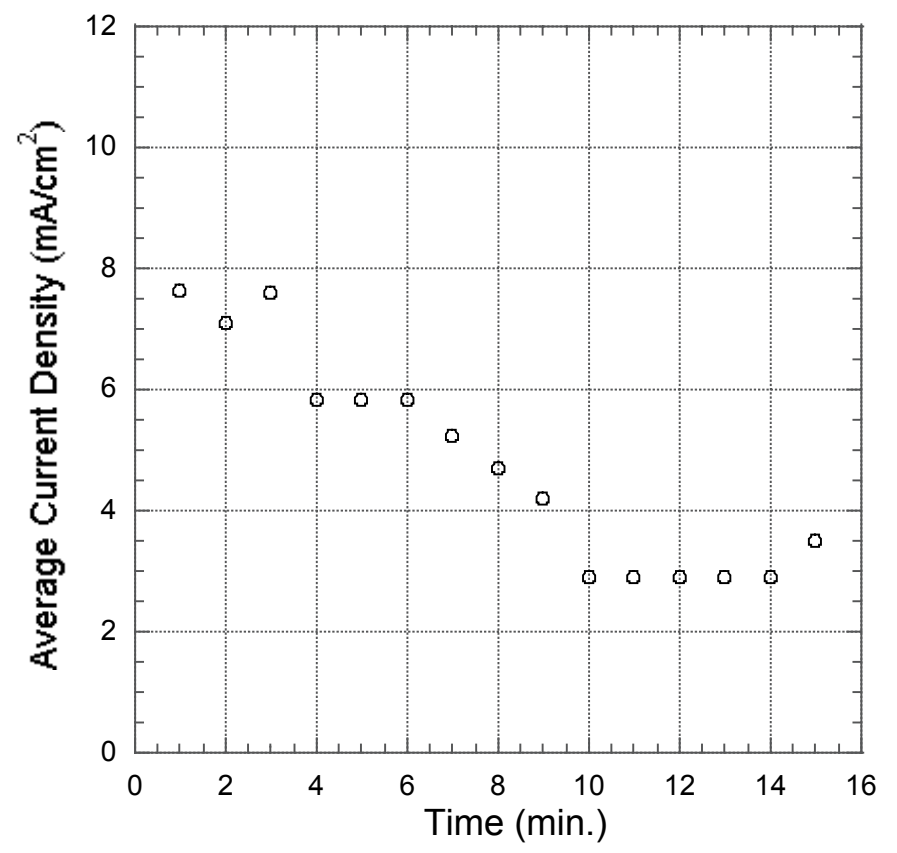

Figure 4.10. Rapid decrease of output current in a potassium doped tungsten source at $1,300^{\circ} \mathrm{C}$. 
In general, the performance of $\mathrm{K}$ doped sources was more difficult to maintain on a long term basis than in the case of Cs as will be shown in the following section. It may be appropriate to mention here that the previous results are consistent with the observations carried out at LBNL's 2 MV injector, where the large (4 and 6 inches diameter) contact ionization sources usually provide up to a week of operation (in cycles of $\sim 8 \mathrm{hrs} /$ day for one week).

\subsubsection{Performance of High Current Density Cesium on Tungsten Contact Ionization Sources.}

A test under very close conditions to the one just described above is presented for the case of a cesium on a porous tungsten emitter. The initial performance, with the maximum output, of such a source is shown in Figure 4.11. The $\mathrm{V}^{3 / 2}$ line corresponds to the expected Cs output based on the expected scaling of the emitted current given the different mass of the Cs ion. The behavior of the curves does not follow as closely the space charge limit as in the shown examples with potassium sources in this particular case. As it can be seen in the subsequent graphs, this feature is accentuated after the source has been operated continually for a number of hours. Despite maintaining similar heating cycle conditions as the potassium doped source of the previuos section, the Cs source shows features in its behavior that are unusual but nevertheless worth mentioning. 


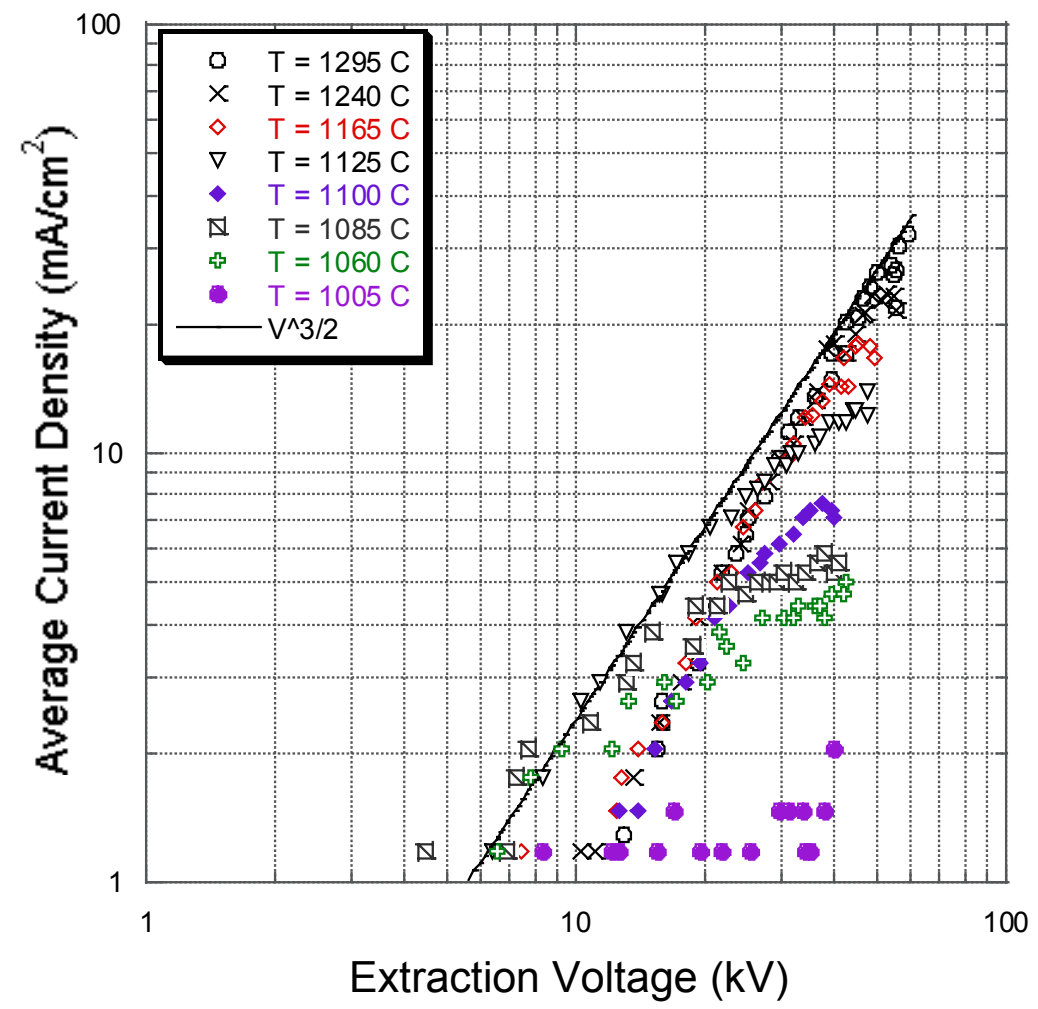

Figure 4.11. $\mathrm{J}-\mathrm{V}$ characteristics of a recently doped cesium on tungsten contact ionization source.

The main feature was a decrease in the ion yield in a time span of approximately 10 hours while left operating at a fixed extraction voltage and a temperature of $1,090{ }^{\circ} \mathrm{C}$. This is shown in Figure 4.12. The relevance of this is not the apparent accelerated rate of alkali atom loss, since as it will be shown, the ion source performance can be recuperated to some extent by further cycling the emitter at higher temperatures. This suggests some poisoning mechanism that was not duly tracked down during the experiment. No significant changes in the background pressure were detected, although since this parameter was not continually logged, such event can not be fully discarded. 


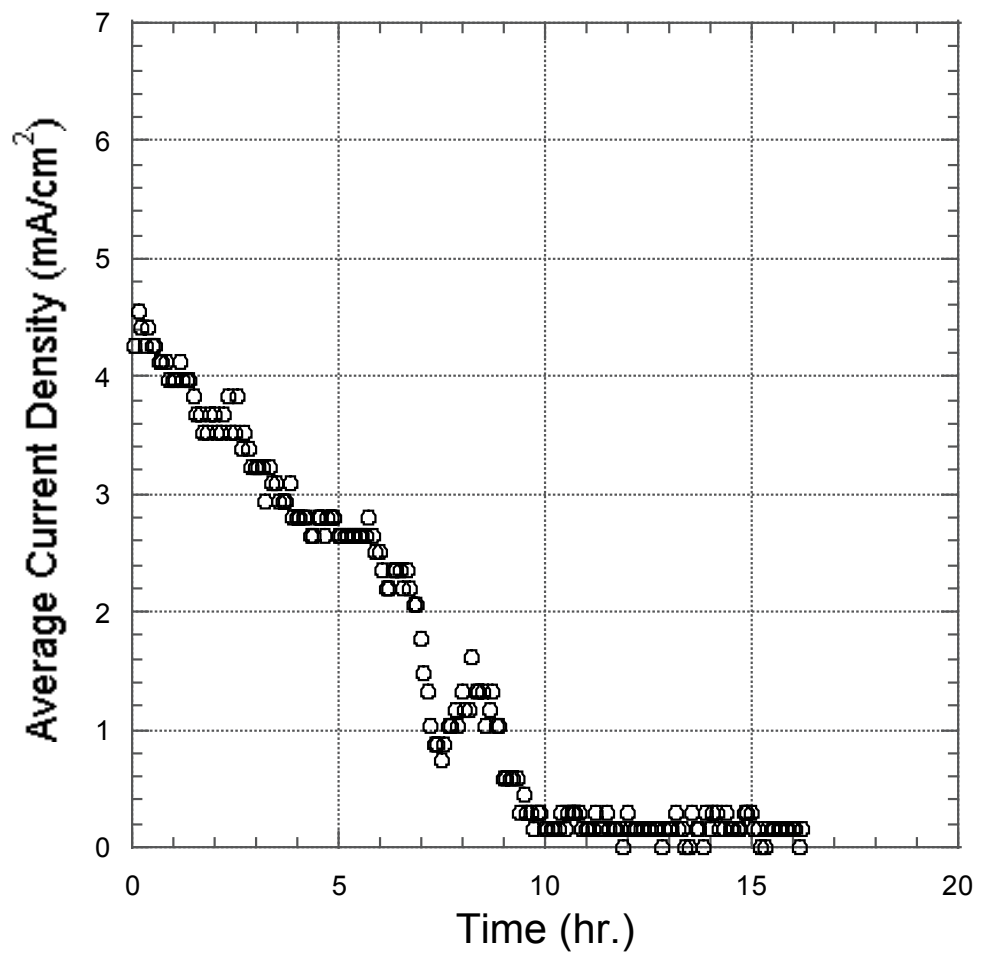

Figure 4.12. Current output at a constant extraction voltage of a $\mathrm{Cs}$ on $\mathrm{W}$ source at $\mathrm{T}=$ $1,090{ }^{\circ} \mathrm{C}$.

Upon increasing the temperature of the emitter beyond $1,200{ }^{\circ} \mathrm{C}$, it was possible to recover part of the lost performance. The new $\mathrm{J}-\mathrm{V}$ characteristics are shown in Figure 4.13. It can be seen now how the source behaves in the emission limited mode of operation regardless of the applied extraction voltage. A more common mode of operation for a Cs doped source is depicted later in this chapter. 


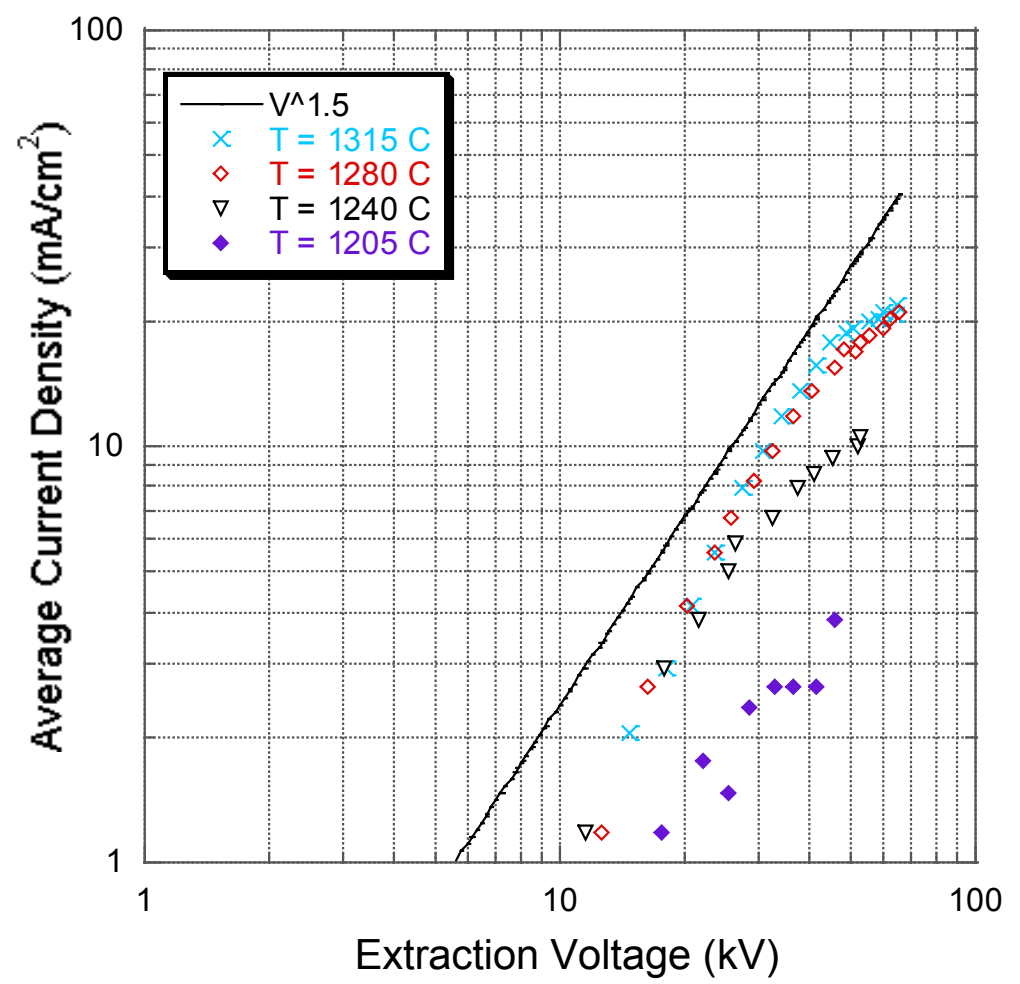

Figure 4.13. $\mathrm{J}-\mathrm{V}$ characteristics of a cesium doped tungsten source after $18 \mathrm{hr}$. of operation at $1,100{ }^{\circ} \mathrm{C}$ and reactivation after the ion output previously decayed to zero.

The last stage of the cycle was performed in an analogous manner with the last stage for the potassium source, a period of more than 30 hours at a temperature of 1,100 ${ }^{\circ} \mathrm{C}$. The source characterisistics are shown in Figure 4.14. Despite a steady decline of the ion output over this period, the average current stayed always above $1 \mathrm{~mA} / \mathrm{cm}^{2}$, increasing with temperature as shown. 


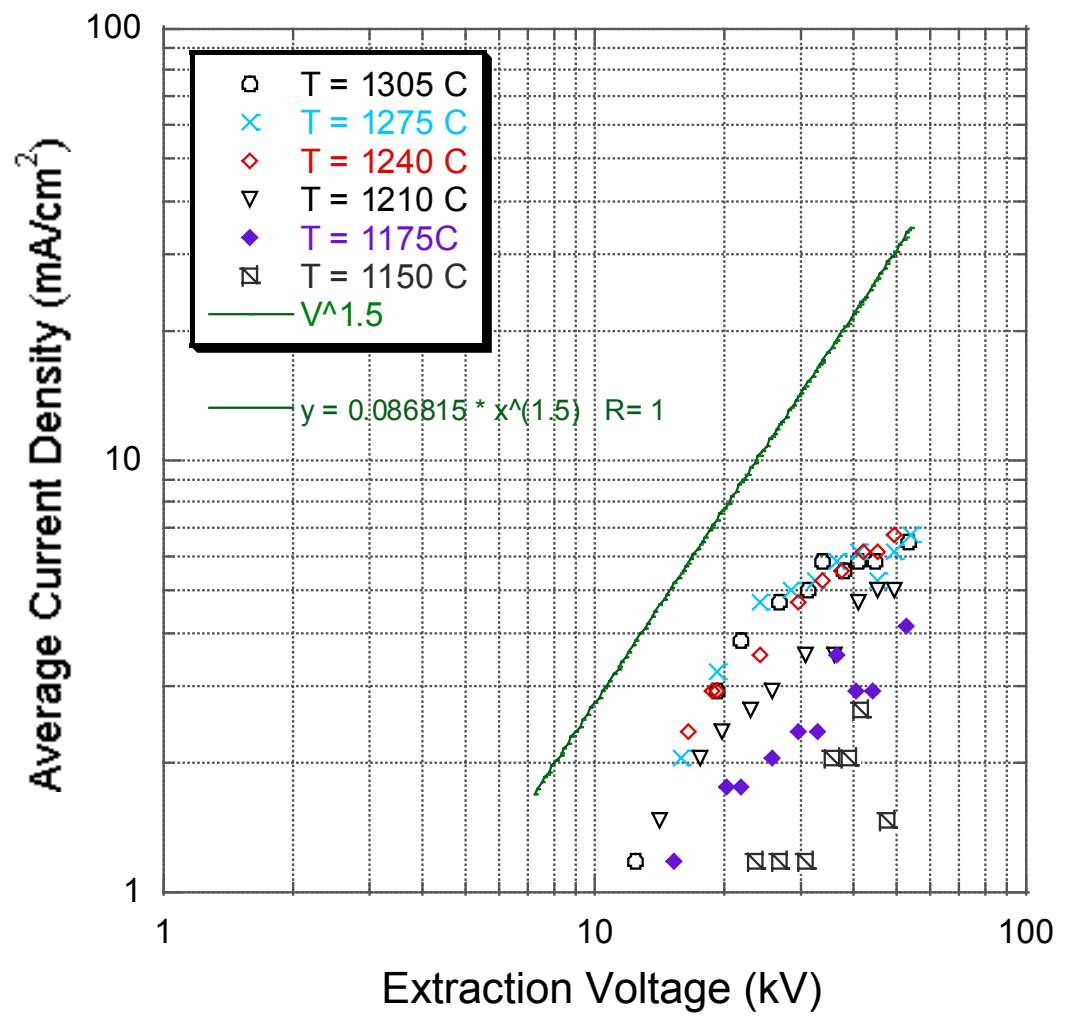

Figure 4.14. $\mathrm{J}-\mathrm{V}$ characteristics of a cesium doped tungsten source after almost $50 \mathrm{hr}$. of operation at $1,100{ }^{\circ} \mathrm{C}$.

Further testing indicated that the variability in the output of the contact ionization sources operating at regimes greater than $\sim 10 \mathrm{~mA} / \mathrm{cm}^{2}$ can be decreased by increasing the time the source spends in the range of up to $\sim 1,050{ }^{\circ} \mathrm{C}$, before demanding the high current densities. Presumably, such pre-heating may allow the conditioning of the surface, evaporating impurities present that may affect the surface properties of the tungsten. At the same time that the internal coverage of alkali atoms is improved. An example of such preliminary heating is seen in Figure 4.15. This graph displays the hours 12 through 16.5 of a source that has been operated continually at a temperature of $1,060{ }^{\circ} \mathrm{C}$. The output is not completely steady. Some of this variation (the small spread or line thickness in the main trend of the data) can be ascribed by a shot-to-shot variation of the applied extraction voltage. The longer-term variations seem to be a characteristic 
of surface conditions that may change on the time-scales suggested by the graph. The full history of the emitter output as a function of time is shown in Figure 4.16. The ion output capability at moderate regimes can be fully appreciated when considering that current densities above $15 \mathrm{~mA} / \mathrm{cm}^{2}$ were maintained for a period of about 70 hours.

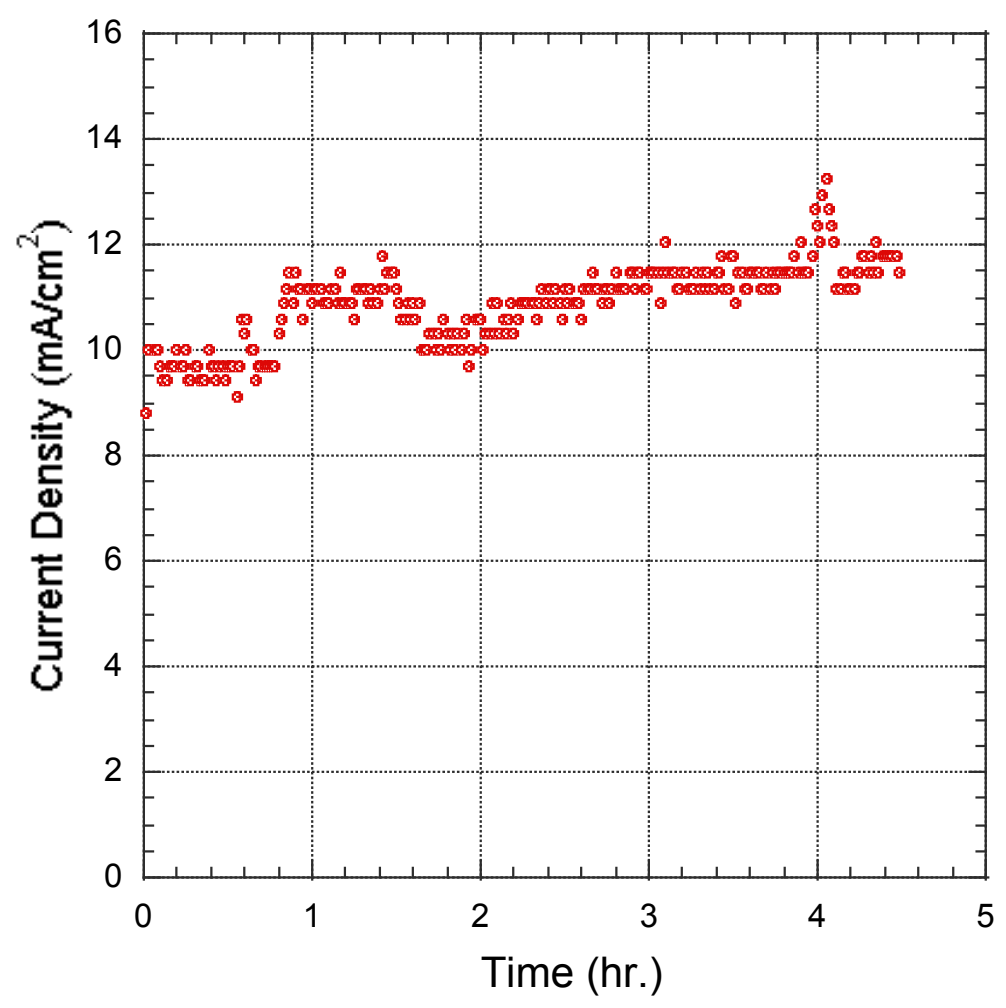

Figure 4.15. Average current output of a Cs source at a constant extraction voltage and temperature of $1,060^{\circ} \mathrm{C}$.

The steady output and relatively long depletion time of the source is consistent with the fact that this source was not subject to the high temperature excursions that were mentioned above. Figure 4.16 displays the typical performance that is to be expected from a contact ionization source operating at a single temperature. The period of steady increase in the output current occurs while the alkali atom content of the surface decreases due to the combined effect of neutral atom evaporation and ion elimination due 
to the applied extraction voltage. Ion evaporation is favored by the decrease in the alkali atom coverage of the surface, hence producing an increase of the current output with time. A "plateau" region is attained at a working point that is a balance between the average desorption rate of the ions and the neutral particles and the average arrival rate of these same species from the bulk of the emitter by diffusion. The third phase represented in Figure 4.16 is the one where the ion output decreases. This corresponds to the depletion region where the ion coverage steadily decreases and is beyond the optimum ion coverage. Any loss of neutral particles as well as any loss of ions is immediately accompanied by a decrease in the surface coverage as the replenishment rate does not compensate for the loss rate.

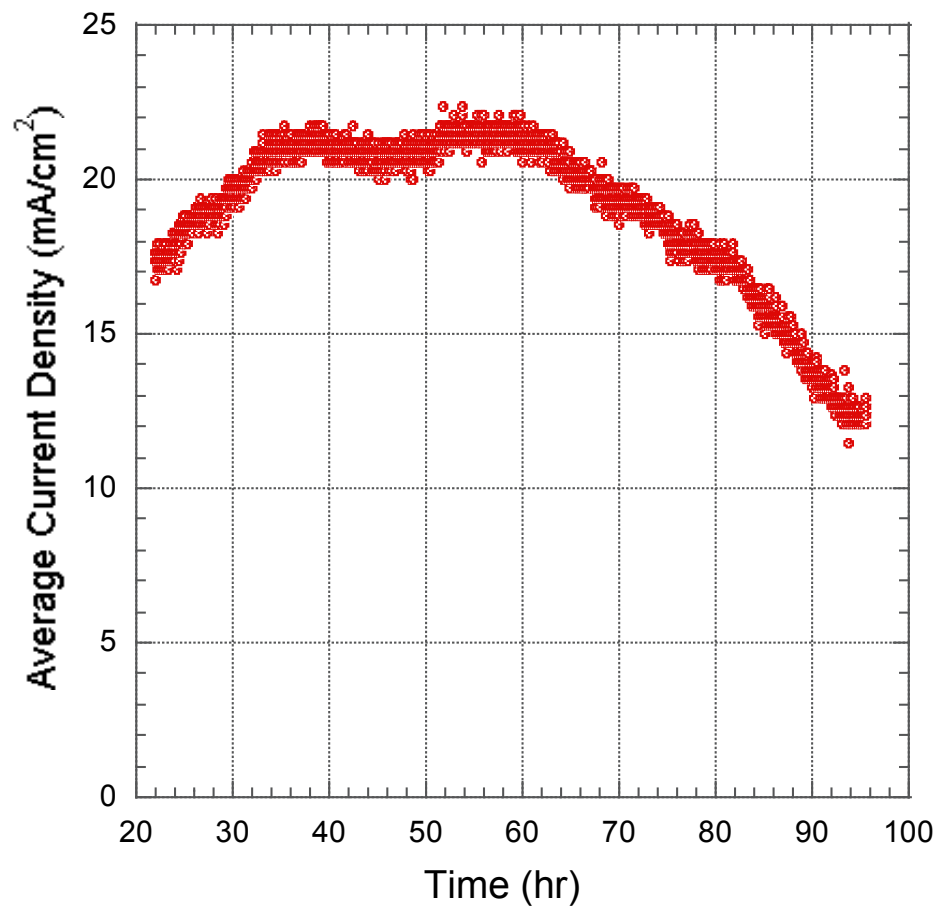

Figure 4.16. Average current output of a Cs source at a constant extraction voltage and temperature of $1,120^{\circ} \mathrm{C}$.

A relatively unusual phenomenon was observed during the measurement being described that illustrates the influence of the extracted current level on the actual ion 
surface coverage. Figure 4.17 shows a measurement of the $<\mathrm{J}>$ vs. V characteristics made in the first stage (during which the output current increases). This figure shows a hysteresis effect that occurs as the extraction high voltage is increased, and thus the amount of ions in the surface is significantly decreased during the pulse. The test was made with a short pulse ( 2.5 $\mu \mathrm{s})$, making the influence of the desorbed ions appreciable. The curve was traced by increasing $\mathrm{V}$ up to a value where the output current was clearly emission limited, following the lower arrow. Upon maintaining the maximum voltage for a few tens of shots, the re-tracing of the curve in the direction of the decreasing voltage yielded a consistently higher current output, as indicated by the upper arrow.

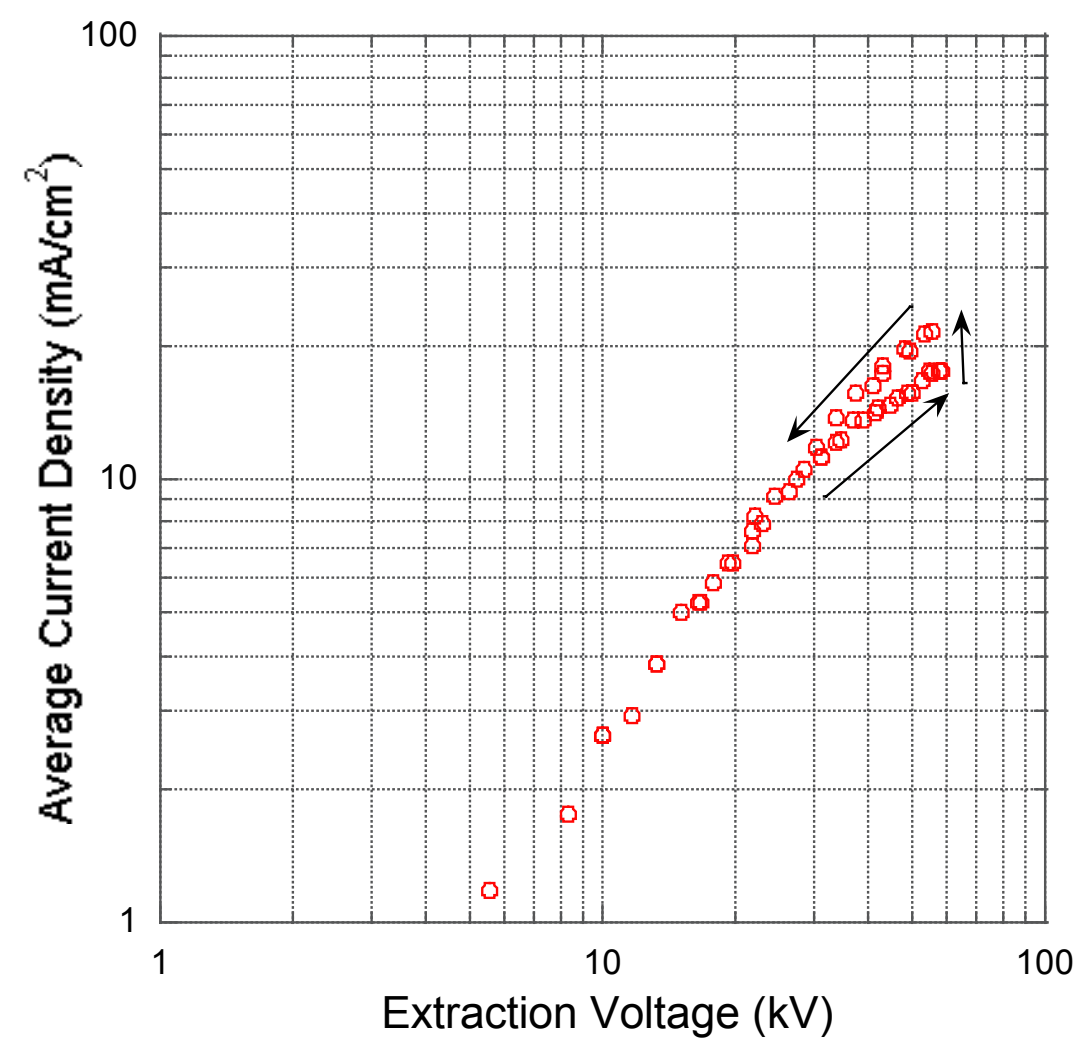

Figure 4.17. Hysteresis effect caused by the influence of the ion desorption on the surface coverage. Operating temperature $=1,120^{\circ} \mathrm{C}$. 
Figure 4.18 shows a collection of the $\mathrm{J}-\mathrm{V}$ curves measured at different points during the life of the source. Optimum source performance can be seen that occurs during the plateau of Figure 4.16 and is represented by the "after 50 hours" curve of Figure 4.18.

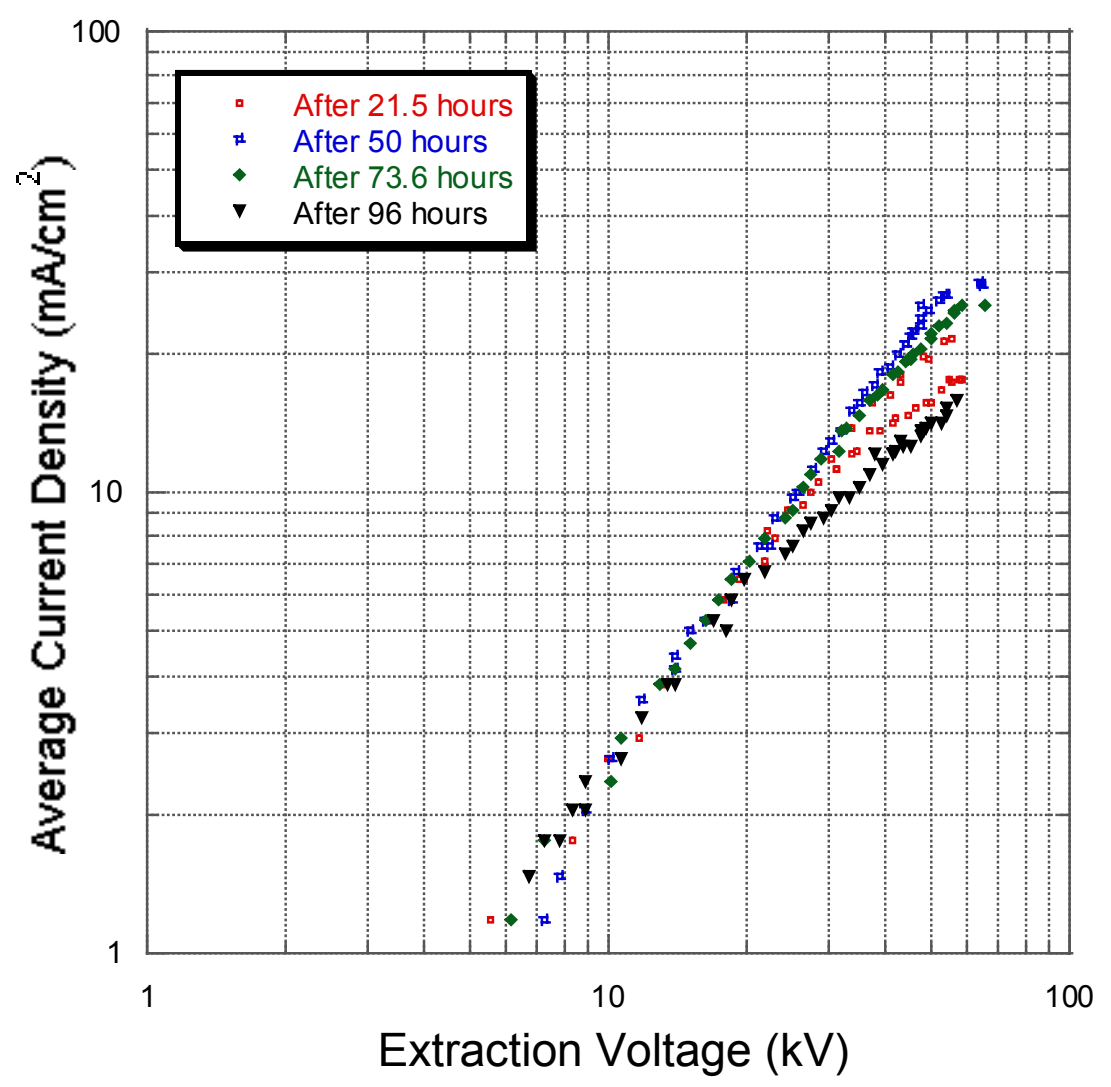

Figure 4.18. Family of $\mathrm{J}-\mathrm{V}$ characteristics measured at different points of the lifetime test presented in Figure 4.16.

Another feature worthy of consideration was observed in this particular run after the cooling down of the source. The emitter was exposed to air and re-doped with a similar amount of cesium carbonate as in the test just described. Once again, the source was subject to a preliminary "pre-heating" cycle of 2 days of operation at a temperature of $985^{\circ} \mathrm{C}$. After this period, the $\mathrm{J}-\mathrm{V}$ characterisitic of the source was as presented in Figure 4.19. The sometimes "capricious" nature of these emitters, despite the apparent maintenance of "standard" conditions is illustrated in what follows. 


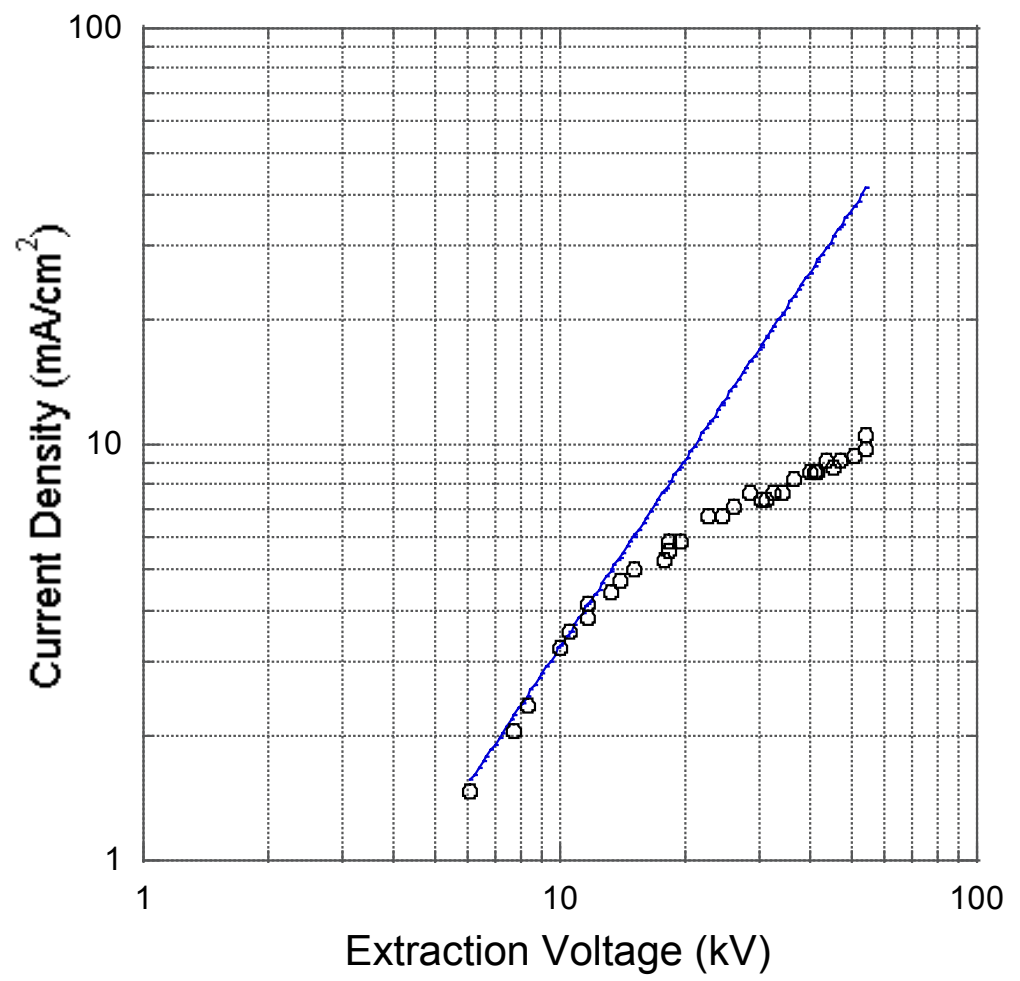

Figure $4.19 \mathrm{~J}-\mathrm{V}$ characteristic of a re-doped Cs on $\mathrm{W}$ source after 2 days of pre-heating at $985^{\circ} \mathrm{C}$.

The curve in Figure 4.19 is in a slightly different horizontal position due to the use of a slightly different high voltage gain factor than used in the previous test, inducing a small translation of the curve to the left, however, this detail is of no relevance to the present discussion. A current extraction test at a constant voltage and at $\mathrm{T}=985{ }^{\circ} \mathrm{C}$ indicated a continual decrease with time of the output current. The same general trend occurred as the source temperature was raised further. In a way, the source was repeating the behavior that would be expected if the emitter had not been re-doped. The actual behavior observed at constant extraction voltage and different temperatures is presented in Figure 4.20. The increase in current output whenever there is a change in temperature can be seen, accompanied by an unexpected decrease with time. Unexpectedly, an internal thermal short decreased the source temperature abruptly to $1,005{ }^{\circ} \mathrm{C}$, with a 
dramatic decrease in the current output as a consequence. A similarly abrupt correction of this problem increased the operating temperature beyond $1,100{ }^{\circ} \mathrm{C}$.

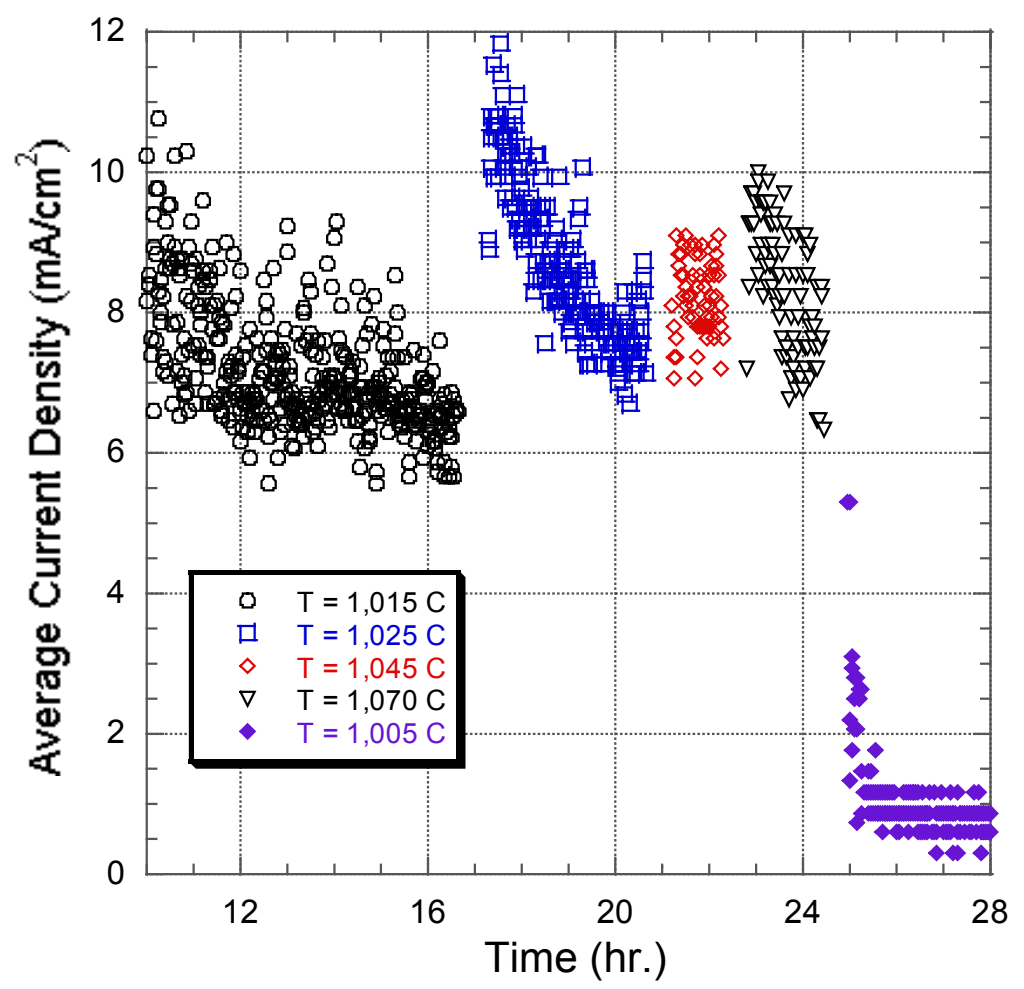

Figure 4.20 Output vs. time at a constant voltage of a re-doped Cs on tungsten emitter, displaying an unexpected decrease of output with time.

The dramatic activation of the source at this temperature can be appreciated in Figure 4.21. It was an unfortunate circumstance in this run that the erratic source heating behavior occurred, as it cannot be demonstrated that the full reactivation of the source is unrelated to the increased time of operation at temperatures above $1,000{ }^{\circ} \mathrm{C}$. However, a reasonable number of temperatures in the interval 1,000 to $1,100{ }^{\circ} \mathrm{C}$ were explored (Figure 4.20) with no indication whatsoever of a source activation. Given the rapid increase in output current observed at temperatures beyond $1,100{ }^{\circ} \mathrm{C}$, there is evidence that Cs requires an operating temperature of this magnitude to attain the surface 
conditions that allow for its optimum performance at high current densities. A sample $\mathrm{J}$ - V characteristic of the performance achieved after the activation of the source is presented in Figure 4.22. The straight line in this figure is a reference $\mathrm{V}^{3 / 2}$ line.

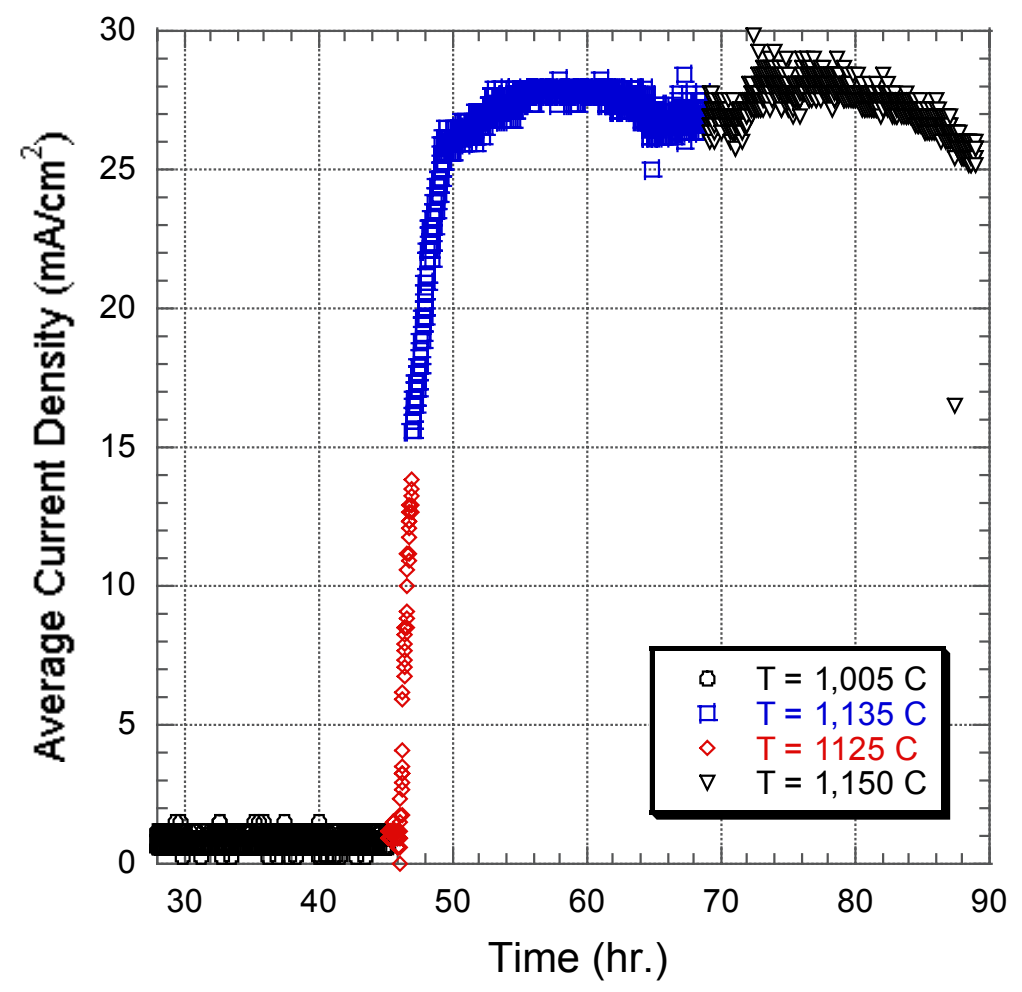

Figure 4.21 Abrupt activation of a $\mathrm{Cs}$ on $\mathrm{W}$ source after its operating temperature went beyond $1,100{ }^{\circ} \mathrm{C}$.

Upon attaining the maximum performance conditions, and just when the first signs of source decay in output were being observed, it was possible to back down the operating temperature to assess the maximum average current available at different operating temperatures. These results are presented in Figure 4.22. The current density levels shown in this figure are comparable to the levels attained in Figure 4.20, the main differences being the slightly higher current density levels after the full recovery of the source, and most importantly, the constancy of the current level, which was dropping before the source was "activated". 


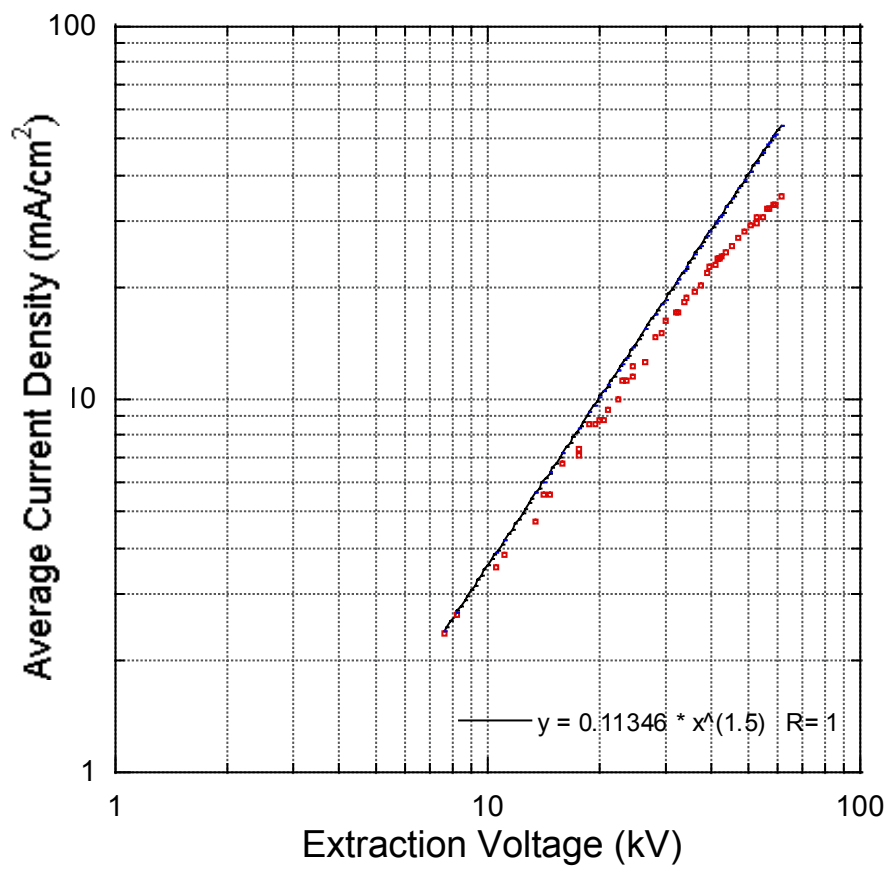

Figure 4.22 $\mathrm{J}-\mathrm{V}$ performance of the activated $\mathrm{Cs}$ on $\mathrm{W}$ doped source at an operating temperature of $1,150^{\circ} \mathrm{C}$.

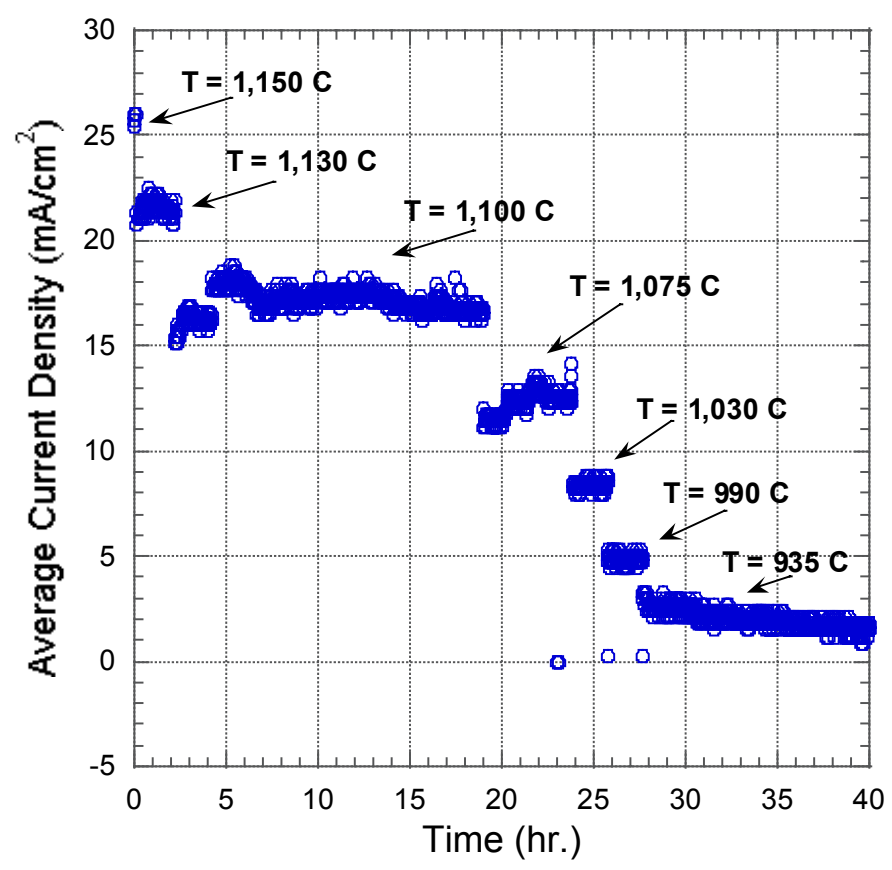

Figure 4.23 Cooling down cycle of a Cs on tungsten source showing the maximum average current densities achievable at the different temperatures in an emission limited mode. 


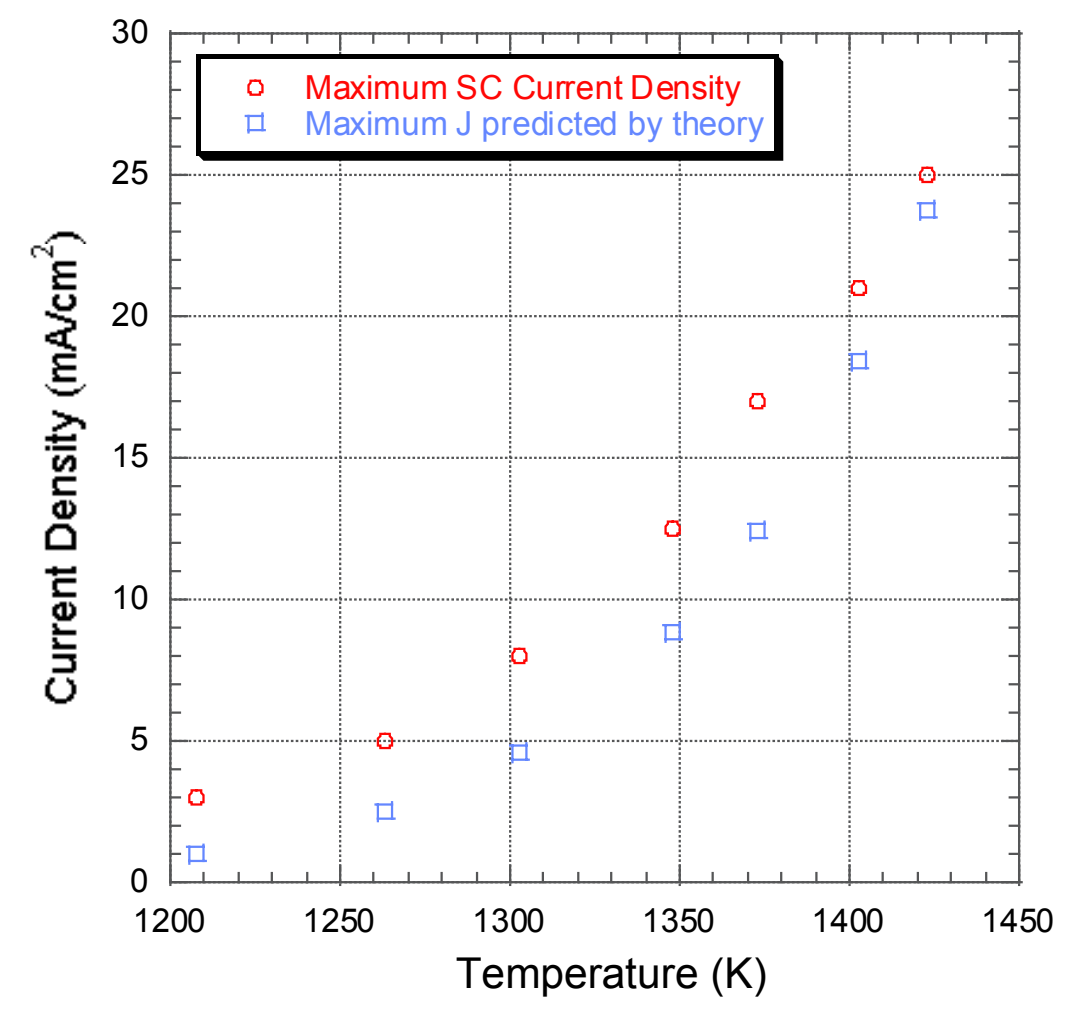

Figure 4.24 Comparison of the maximum average current density measured for the Cs doped source compared with the maximum expected theoretical current density.

\subsubsection{Performance of High Current Density Potassium Aluminosilicate Sources.}

A similar characterization of the performance of a potassium aluminosilicate source as the ones described above was carried out. This type of source has revealed unexpected performance levels in terms of the high current density achieved. As usual, the main diagnostics that were carried out have been a direct measurement of the average current density output at different operating temperatures, as well as a measurement of the current output as a function of time.

This type of source was prepared by the deposition of a thin (approximately 0.7 $\mathrm{mm}(\sim 0.030$ ") $)$ aluminosilicate layer on top of a porous tungsten surface. The tungsten surface is of exactly the same type as the ones used for the doped ion sources described in the previous sections. The layer is deposited in the form of a slurry made by adding de- 
ionized water to the aluminosilicate powder. The appearance of the layer before firing is that of a compact, white, layer whose shape can be easily formed at this stage. Once deposited the layer is allowed to dry and then fired at a temperature between 1,500 and $1,600{ }^{\circ} \mathrm{C}$ in a vacuum furnace. The typical physical appearance of these sources is a glassy finish, with an opaque grayish color.

Figure 4.25 shows a graph of the average current density extracted at a fixed extraction voltage. Once again, the source was pulsed with a very low duty factor, one $2.5 \mu$ s pulse every 20 seconds, with the data being measured once a minute. The graph represents the initial heating cycle, showing the current density level (in the emissionlimited mode) obtained at the same voltage. The various trends that can be identified mostly denote stable output levels in temperatures up to approximately $1,025{ }^{\circ} \mathrm{C}$, with signs of increase with time. A steady increase of current with time was achieved at a temperature of $1,100{ }^{\circ} \mathrm{C}$. 


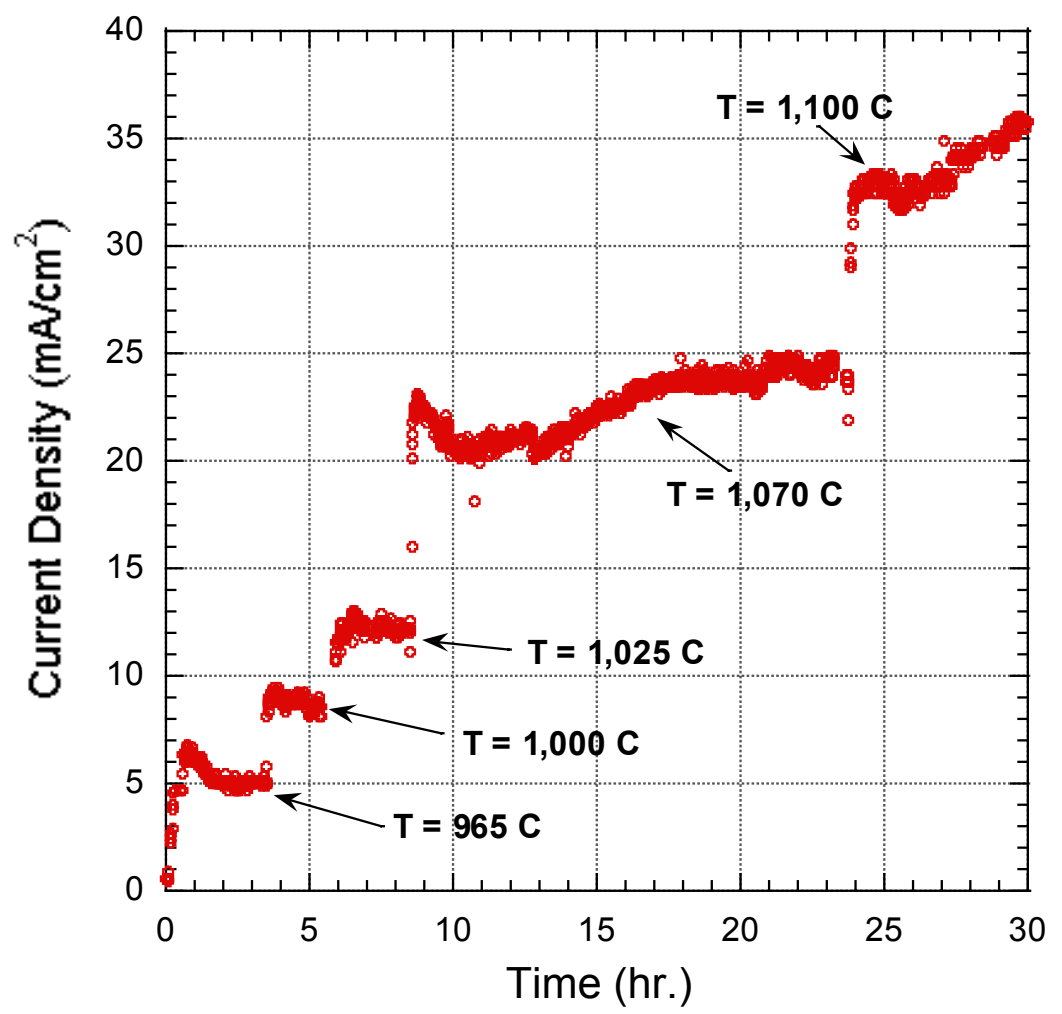

Figure 4.25 Average current density variation with time during the heating-up cycle of a potassium aluminosilicate source, at different operating temperatures and fixed extraction voltage.

Figure 4.26 shows a more detailed history of the current density output fluctuations occurring at $1,100{ }^{\circ} \mathrm{C}$. The graph is the immediate continuation of Figure 4.25. The usual spread of the data points around the main line can again be ascribed to small shot-to-shot variations of the applied high voltage. Current fluctuations in longer time scales are presumably a characteristic of the ion emitter. Of particular interest here are the discontinuities that arise at a few positions, such as at Time $=46$ hours and Time $=105$ hours. These discontinuities have occurred at points during the life test at which $\mathrm{J}$ - V characterizations have been made. Measures were taken to ensure a return to the same fixed extraction voltage upon returning to the $<\mathrm{J}>$ vs. time measurement, so this event can be discarded. 


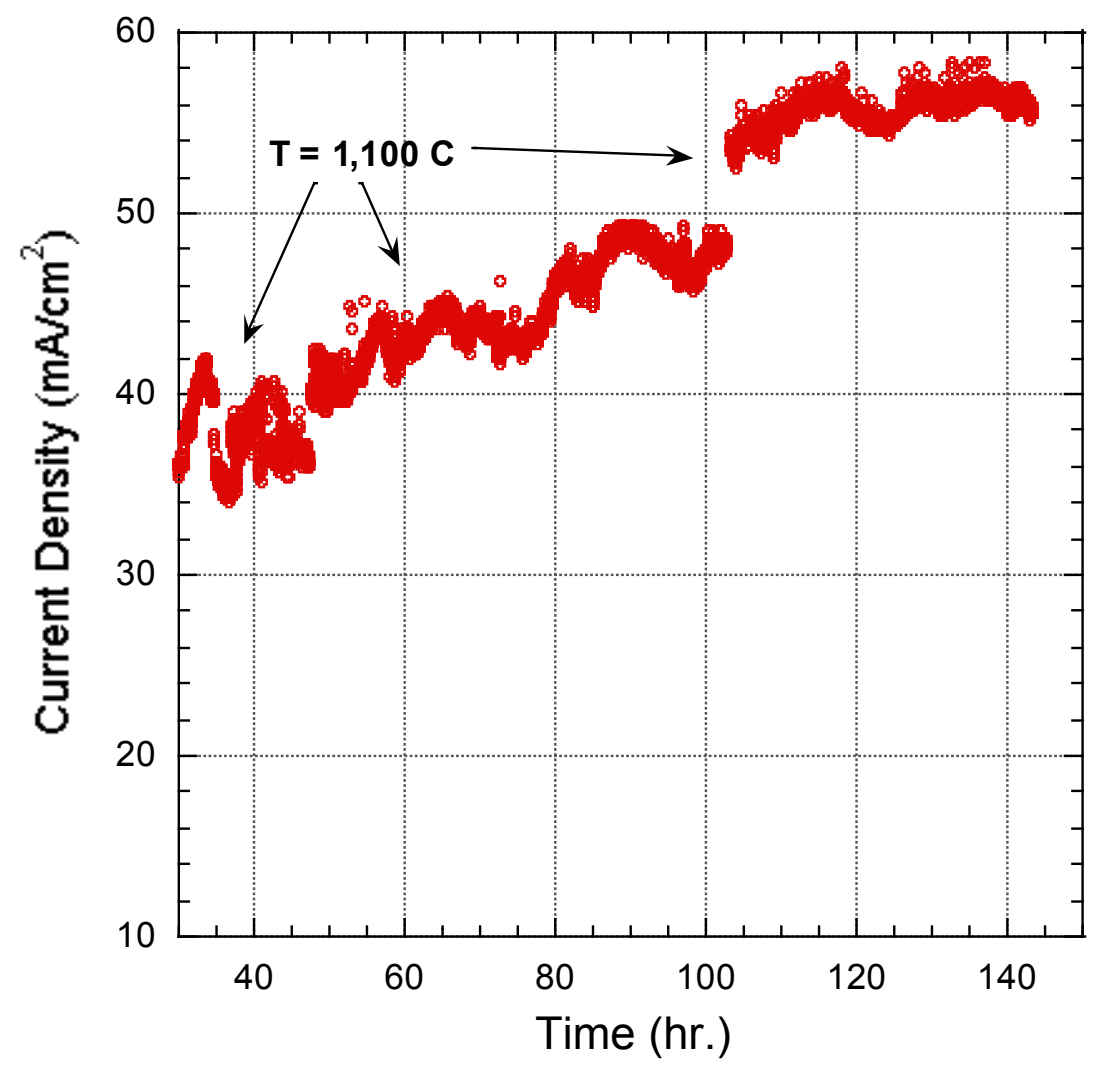

Figure 4.26. Continuation of the life test of figure 4.27 showing increased current output with time at a fixed temperature, and the discontinuities that occur after the emitter has been subject to peak extraction voltages during $\mathrm{J}-\mathrm{V}$ characterization.

In a similar fashion to the hysteresis effect observed for the doped cesium source, the discontinuity occurs just after the emitter has been subject to the highest extraction voltages during the $\mathrm{J}-\mathrm{V}$ measurements. This seems to be one more similarity between the workings of contact ionization sources and aluminosilicate sources. The fact that similar ion species can be produced with the silicates and the doped sources suggests an analogous mechanism for ion production based on the interplay between the low ionization potential of the alkalis and the effective work function of the mineral structure. Taking this analogy a step further, it would be reasonable to expect that the atomic concentration of the alkali atoms in the first few layers of the aluminosilicate surface could influence this "effective" work function, thus changing the overall ion availability 
for extraction. (It will be seen in Chapter 5 that in the case of the doped sources, the concentration of alkalis has a strong influence on the surface's work function.) The increased ion yield after the source has been subject to the peak extraction voltages may be an indication of this phenomenon. In principle, high $\mathrm{J}$ pulses have the potential to affect the surface concentration appreciably during the pulse time.

Figure 4.27 shows a collection of measurements representing the $\mathrm{J}-\mathrm{V}$ characteristics of the same emitter. In contrast with the previous cases presented for the operation of doped sources, the aluminosilicate sources display an "activation" phenomenon that occurs not in the scale of hours, but days. For this reason, several curves are presented for the same operating temperature. The difference between the curves is the time during the test at which they were measured.

It can be easily seen how the gun space charge limit is achieved along all the range of applied voltages after the source has been operating at $1,100 \mathrm{C}$ for almost 120 hours. It is easy to appreciate why the aluminosilicate sources are an option of choice in HIF research, given their capability to sustain low average current densities for extended periods of time. This kind of performance can be appreciated in the performance of the large (multi-inch diameter) aluminosilicate emitters used in the $2 \mathrm{MV}$ injetor at LBNL.

The detailed physics of ion emission from an aluminosilicate source is not well understood. As mentioned before, the interaction between the alkali atoms and the anion framework operates analogously with the interaction that happens in doped sources between the alkalis and the metallic substrate. It is known that the aluminosilicate has the ability to regenerate some of its output current even after a forced depletion (See Section 
4.2.4), which suggests either the process of migration of alkali atoms within the framework or the elimination of the outer, depleted layers.

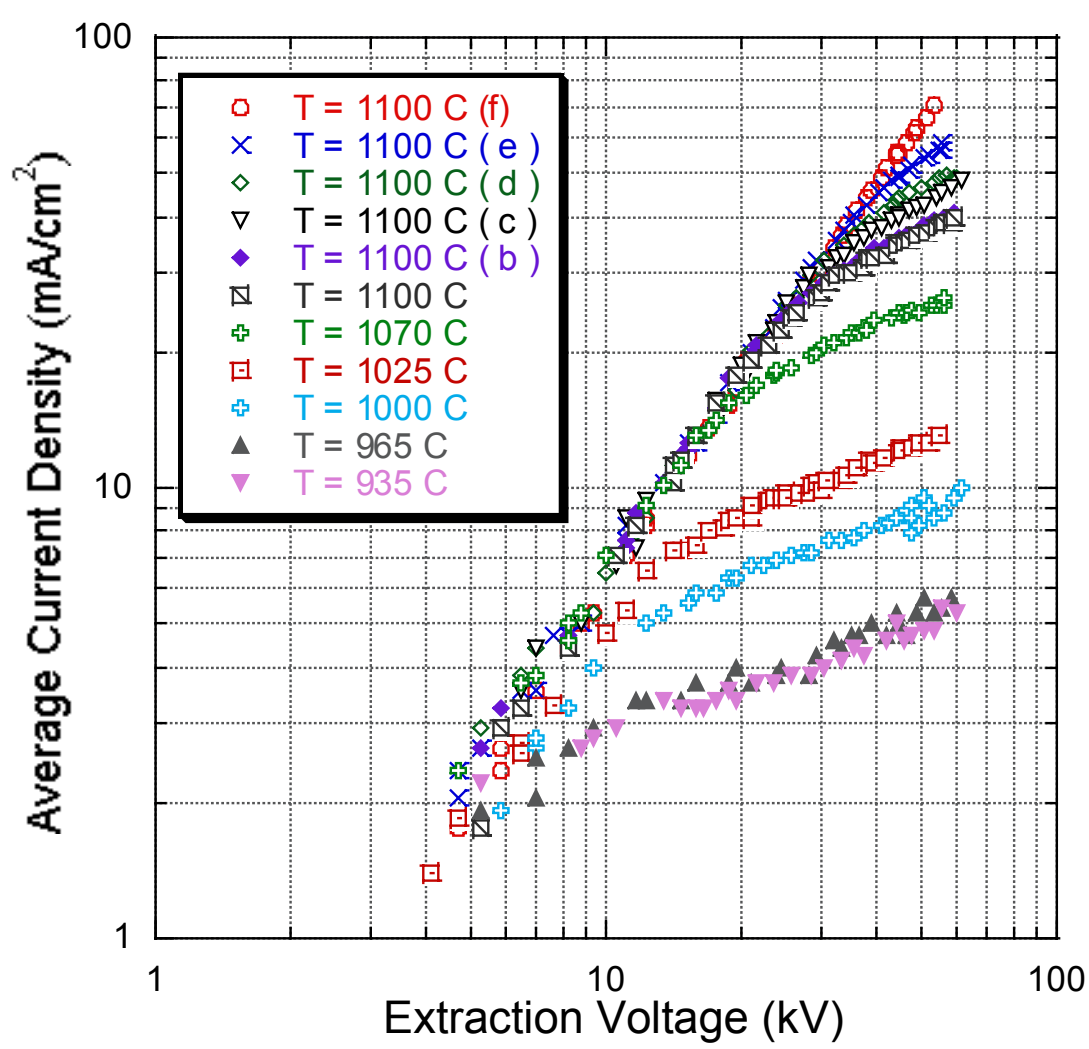

Figure 4.27 Performance characteristics of a potassium aluminosilicate source. Curves (b), (c), (d), (e) and (f) were measured after 24, 28, 48, 78 and $119 \mathrm{hr}$ of operation at $1,100{ }^{\circ} \mathrm{C}$ respectively.

There is evidence for the elimination of radicals from the aluminosilicate, in the form of oxygen, silicon or aluminum ions, or in the form of radicals like $\mathrm{SiO}, \mathrm{SiO}_{2}, \mathrm{AlO}$, etc. This has been observed in other experiments with long transport channels that allow for an identification of the different charge to mass ratio of the impurities (Tiefenback, 1986). In our case it is possible to see deposits of material found in the extractor plate after long periods of operation with this type of source.

The physical changes occurring in the aluminosilicate are evident. Figure 4.28 shows a photograph of two aluminosilicate emitters. Before the measurements both 
emitters had the same appearance as the one being held with the tweezers (with grayish color). After almost 220 hundreds of hours of operation and full "activation" of the source, the color of the material became white as the emitter installed in the module. The nature of these changes is not fully understood. Such changes of appearance and had not been produced before since until these experiments, this material had not been subject to the conditions in temperature and time that these changes require.

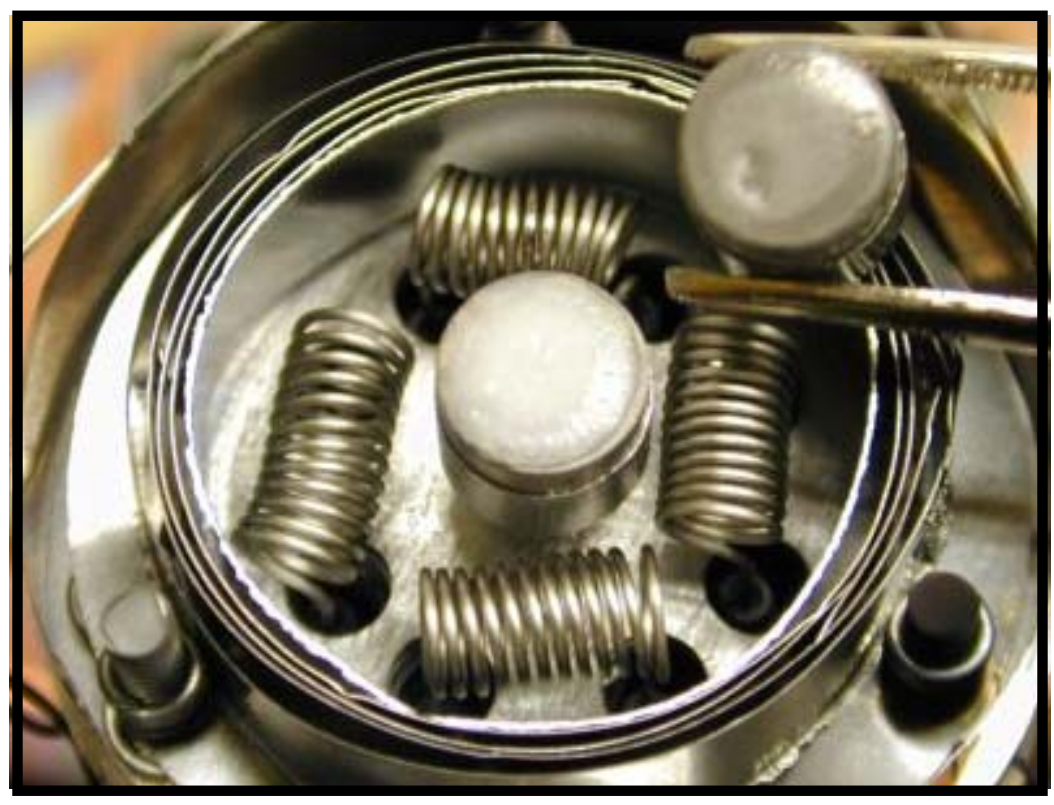

Figure 4.28. Comparison between a recently manufactured aluminosilicate pellet (gray) and a fully activated emitter after $\sim 220 \mathrm{hrs}$. of operation.

The previous test was stopped after an indication of a decrease in the output current density of an approximate magnitude of $5 \%$ over a period of 10 hours. Upon cooling down the source and inspecting it, it was noticed how the long period at high temperature had actually changed the physical position of source components, affecting 
the position of the emitter within the Pierce electrode. Figure 4.29 shows a photograph of the actual droop of the source.

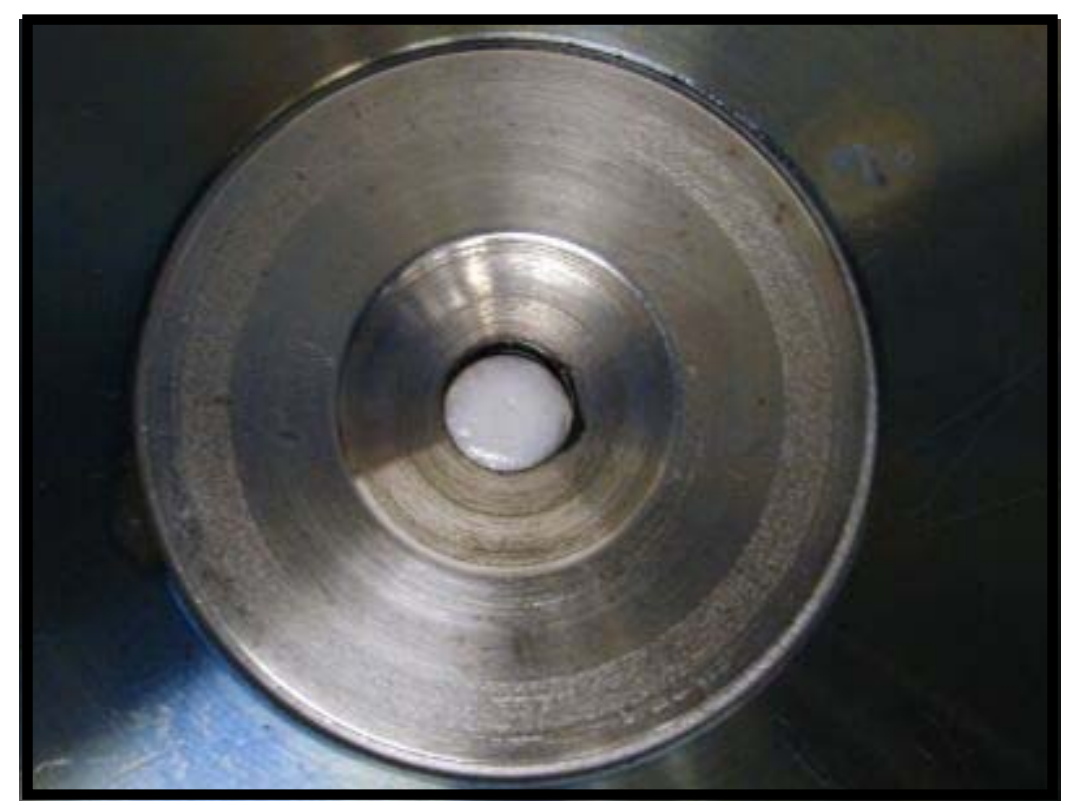

Figure 4.29. Displacement of an aluminosilicate source within the Pierce electrode after almost 220 hours of operation.

The results obtained with this type of aluminosilicate material are not unusual and could be observed with other emitters tested in experiments similar to these. These tests revealed that the aluminosilicate ion sources indeed "activate" over time and are able to deliver increased current density. Figure 4.30 shows another $\mathrm{J}-\mathrm{V}$ characterization of a different potassium aluminosilicate source. The performance is very similar (the difference being slightly lower values for Figure 4.30 at similar temperatures), but the performance once again displays the continual improvement with time until the space charge limit is attained over all the values of the applied extraction voltage. The operating temperature of $1,100{ }^{\circ} \mathrm{C}$ was chosen, as it had already been verified that the 
processes that allow the attainment of high current densities were possible at such temperature.

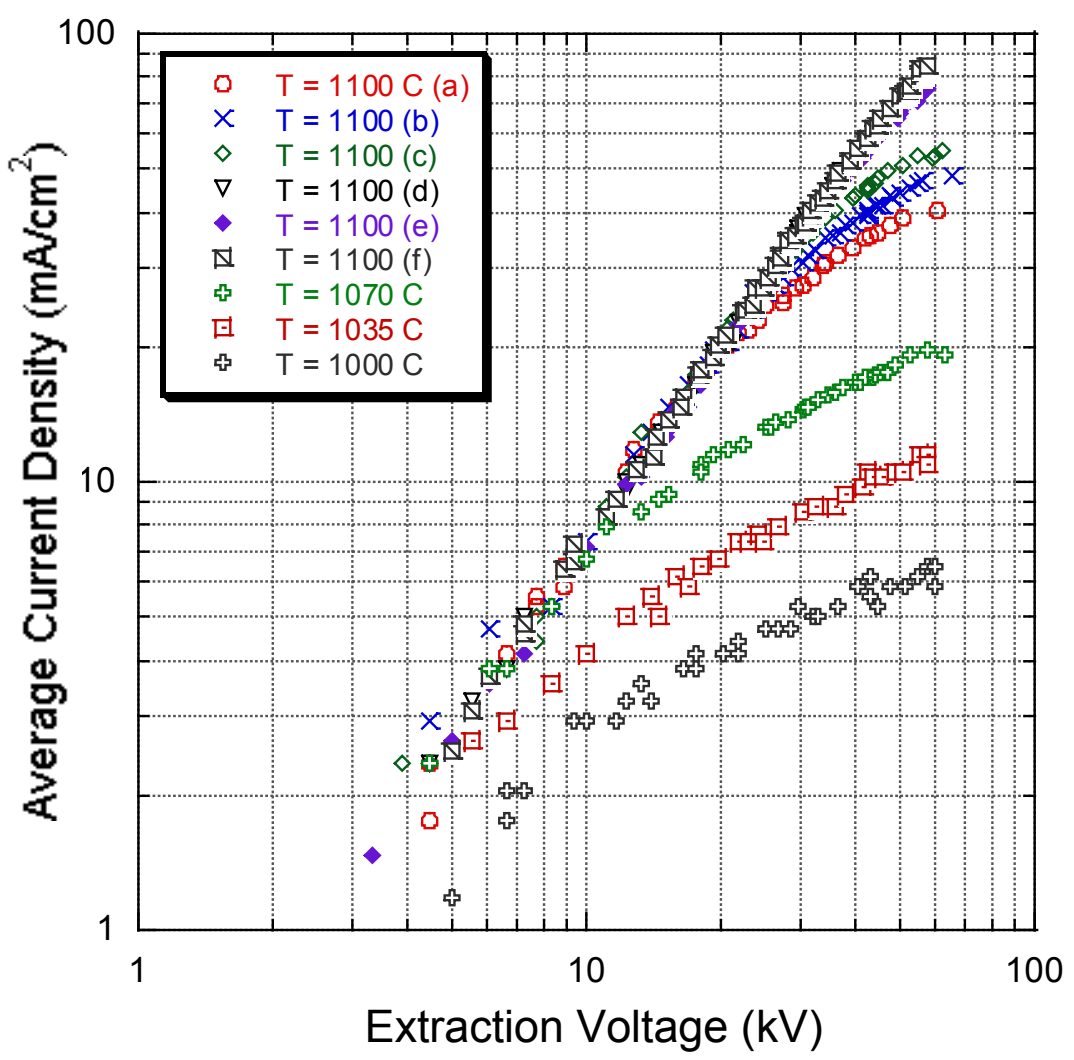

Figure 4.30. Performance characteristics of a potassium aluminosilicate ion source. Curves named (b), (c), (d), (e) and (f) were measured at 24, 48, 72, 120 and 145 hours of operation at $1,100{ }^{\circ} \mathrm{C}$ respectively.

The average current density output dependence with time is shown in Figure 4.31. Once again, the source ion yield increased with time up to the space charge limit of the gun in a period of approximately 5 days, at an operating temperature of $1,100{ }^{\circ} \mathrm{C}$. The source displays the typical erratic behavior in terms of fluctuations of its output current (as explained above), and the discontinuities in current just after the peak extraction currents were applied. 


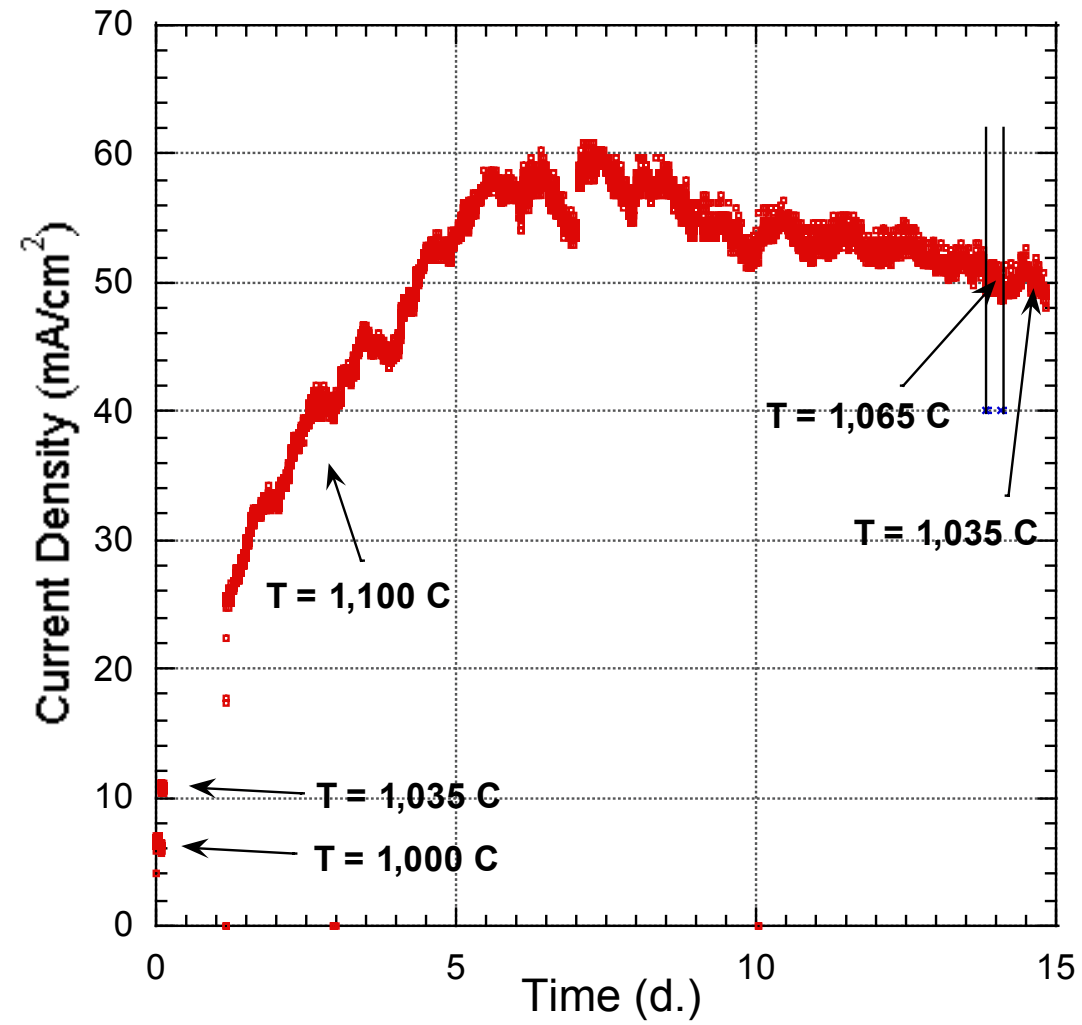

Figure 4.31 Long term time dependence of the average current density output of a potassium aluminosilicate source at a fixed extraction voltage.

A sustained decrease in the output current of the source can be seen to occur after the first week of continuous operation. Nevertheless, the $J-V$ characterizations of the emitter indicated the source capability to provide a space charge limited emission over all the range of applied extraction voltages. Such feature should not be present if the decay in output current were a result of a (partial) depletion of the source. Furthermore, the $\mathrm{J}-$ $\mathrm{V}$ characteristics such as the ones presented in Figure 4.32 indicated a clear translation of the ion source's space charge limit line to lower operating values. 


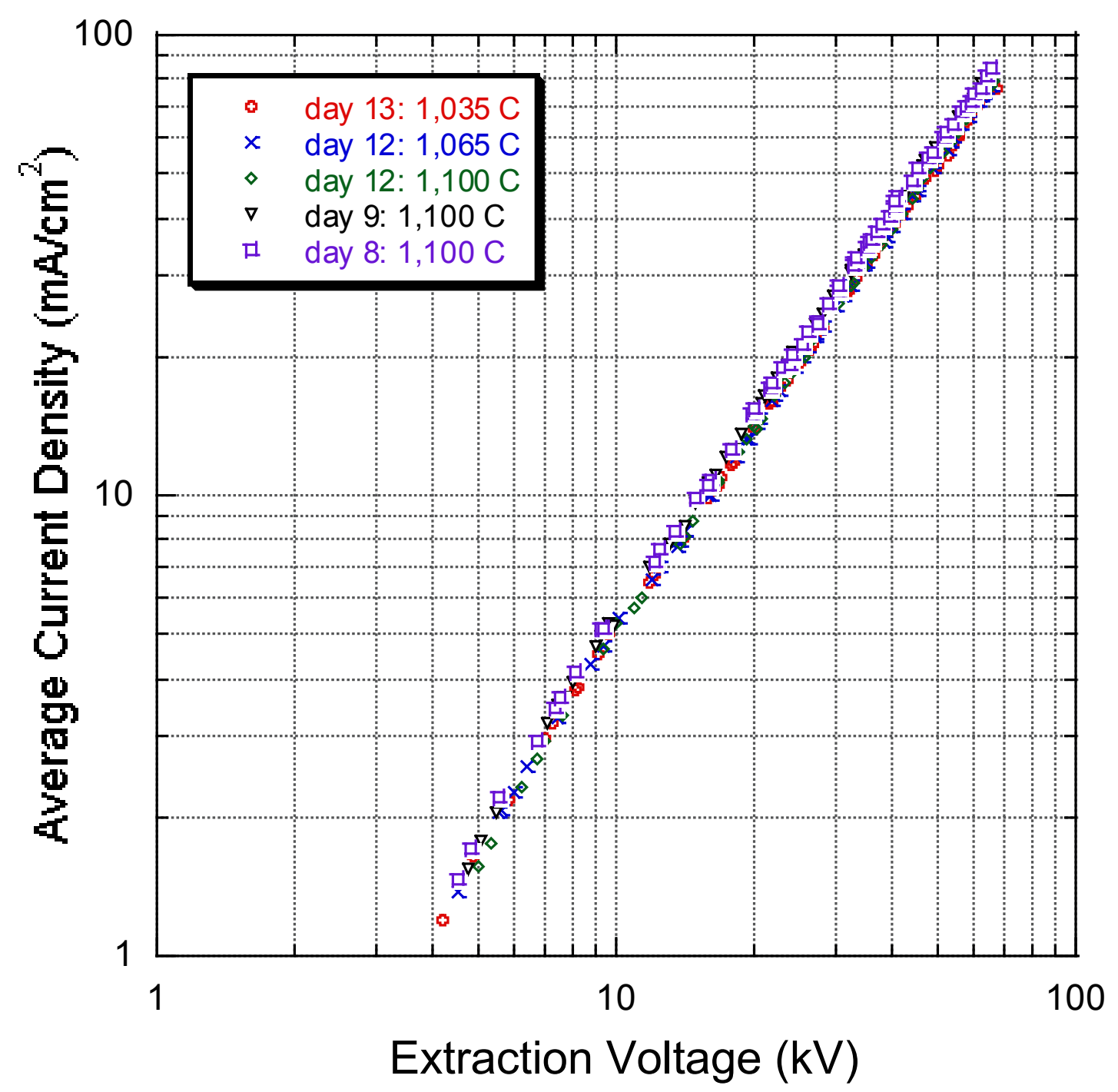

Figure 4.32 $\mathrm{J}-\mathrm{V}$ characteristics of a potassium aluminosilicate emitter showing a vertical translation of the space charge limit line. Note the distinct parallel location of the points measured in the days 8 and 9 of the test in contrast with the data sets taken later.

This behavior is an indication of a change in the ion extraction optics, not of a change in the ion delivery capability of the source. In a similar way to what was seen in the previous long-term testing of an aluminosilicate source, this observation indicated that despite the measures taken to prevent movement of the emitting surface, this was not avoided after all. The emitter holder was strengthened by inserting a molybdenum nut in the base of the threaded stud that keeps the holder in place. Tightening of this nut against 
the heat shields provided an increased stability against lateral movement which was the cause of the decrease in output in the previous tests. Hence, the finding that a similar problem was occurring came as a surprise.

For this reason, the test was stopped to carry out an inspection of the source and the surface conditions of the emitter. The cooling down cycle once again allowed for a direct measurement of the ion yield capability of a fully activated ion source. The results of this measurement are presented in Figure 4.33.

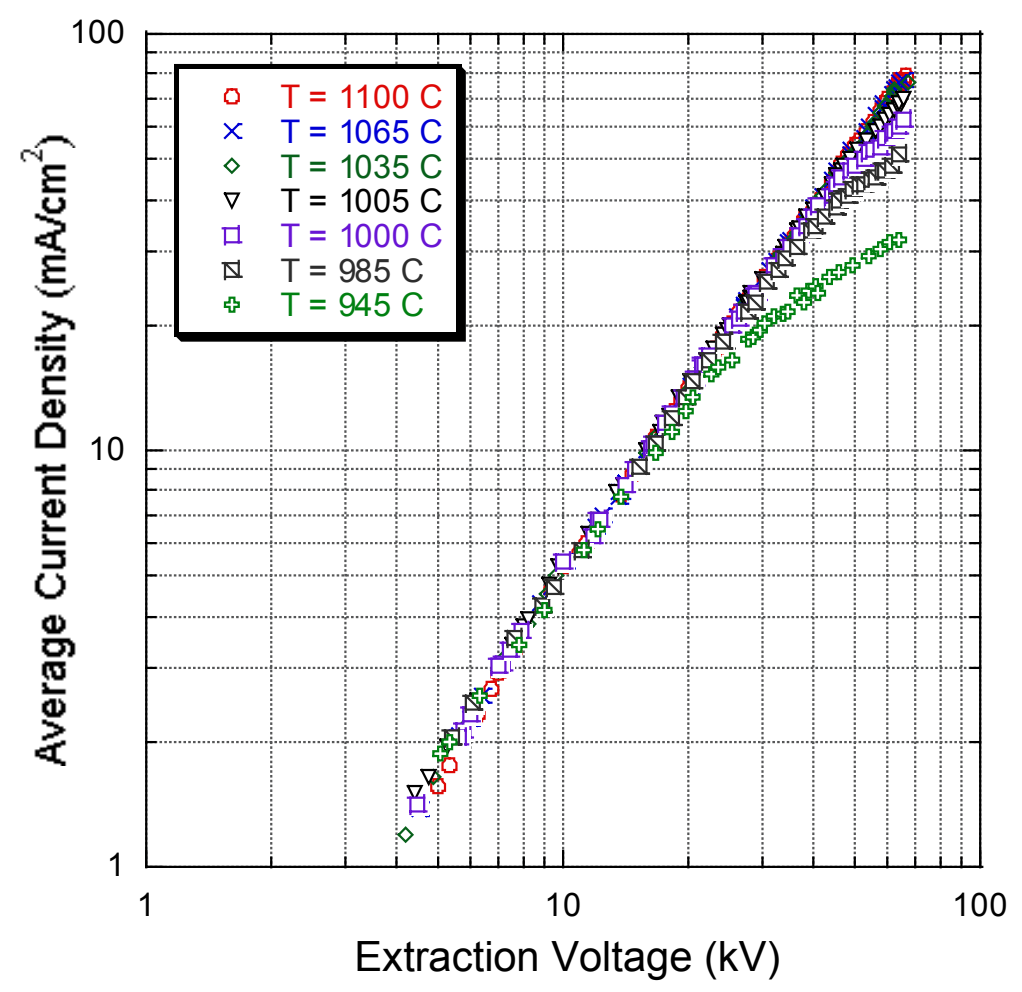

Figure $4.33 \mathrm{~J}-\mathrm{V}$ charateristics of a fully activated potassium aluminosilicate source. Contrast these curves with that of the non-activated source presented in Figure 4.30.

The improvement in the performance of this type of source is quite dramatic when comparing it with the behavior observed at the beginning of the cycle, as shown in Figure 4.30. Note how the relatively cold temperature of $945^{\circ} \mathrm{C}$ is sufficient to sustain space charge limited emission slightly above the $10 \mathrm{~mA} / \mathrm{cm}^{2}$ mark. Even at $1,005{ }^{\circ} \mathrm{C}$, the 
deviation from the space charge limit line is small and only occurring in the highest current densities. In close connection with this, in Figure 4.31 it can be seen how the decrease in source temperature was not accompanied by an immediate decrease in the ion output capability of the source (as can be seen in the case of the tested doped sources, as presented in Figure 4.23 for instance).

Upon taking the emitter out for inspection it was immediately confirmed that there had been a very small change in the position of the emitter within the Pierce electrode. In contrast to the previous test, the change in position of the emitting surface was not a lateral change but a longitudinal (along the direction of the emitted beam) one. The source had retracted a fraction of a millimeter into the Pierce structure. The following two figures show the comparison of the source position "before" and "after" the measurements were made. Figure 4.34 shows the original positioning of the aluminosilicate emitter within the Pierce electrode. Figure 4.35 shows the position of the emitter immediately after the test was suspended.

Figure 4.34 Close-up view of the original position of the K-aluminosilicate emitter in the Pierce electrode. 


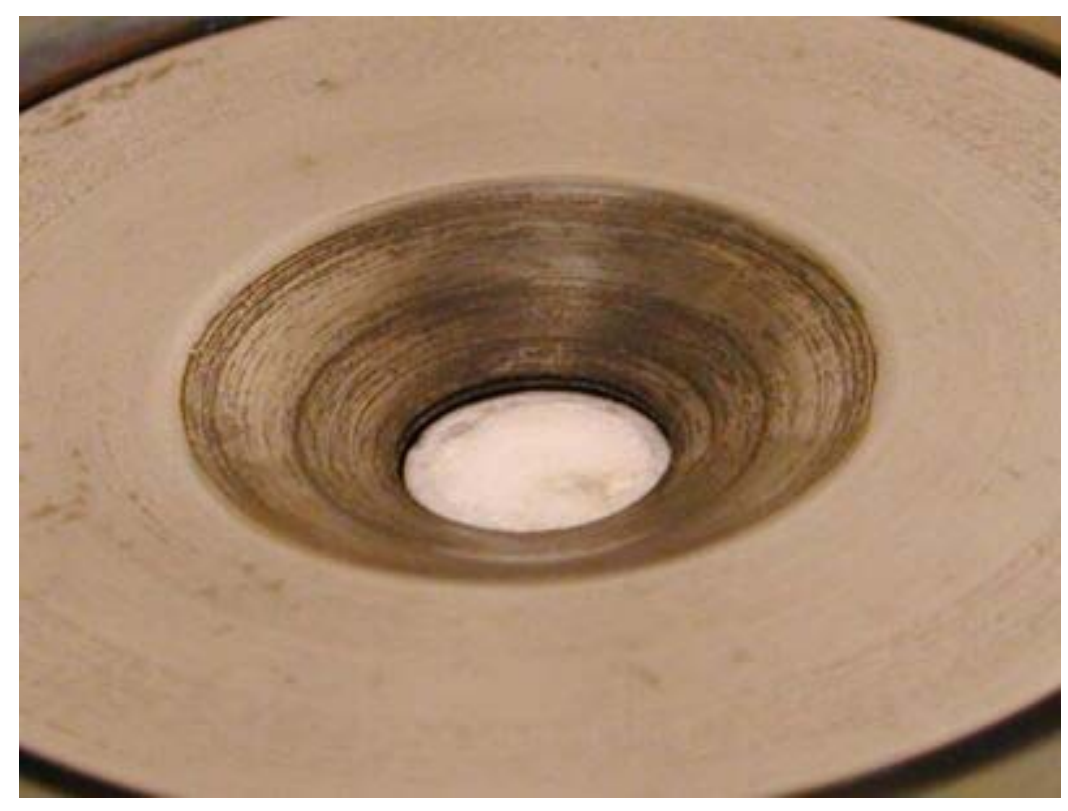

Figure 4.35. Close-up view of the K-aluminosilicate source after almost 15 days of continual operation.

With the small extraction geometry of the source, a small sub-millimeter change in the source position or dimensions is bound to have significant consequences. We only have to consider that the distance existing between the Pierce electrode and the extraction electrode is approximately $8 \mathrm{~mm}$. This would be the "extraction gap" that would be used in a simplified calculation of the magnitude of the extracted current by the onedimensional Child-Langmuir law. The observed change in the longitudinal position of the emitter surface was approximately $0.6 \mathrm{~mm}$. Since the extracted current density scales in inverse proportion to the square of the emitter gap, it should be expected that the emitted current density should change by a factor of

$$
\left(\frac{d_{\text {initial }}}{d_{\text {final }}}\right)^{2}=\left(\frac{d_{\text {initial }}}{d_{\text {initial }}+\Delta}\right)^{2}=\left(\frac{8}{8+0.6}\right)^{2}=0.87
$$


which is in rough agreement with the fraction of available current density at the end of the experiment with respect to the peak value observed. Compare the change in output current density from $59 \mathrm{~mA} / \mathrm{cm}^{2}$ in day 7 of the test, with the final yield of $50 \mathrm{~mA} / \mathrm{cm}^{2}$, as presented in Figure 4.32, for a percentual change of $15 \%$.

The physical changes observed in the emitter this time were not limited to the color of the emitter, but also extended to its texture and thickness. There was an apparent loss (thinning of the layer) of material especially at the edge of the source. Much of the original glassy look was substituted by a duller appearance, almost matte-like. Upon removing the Pierce electrode, the contact between the electrode's knife edge and the aluminosilicate was able to chip the aluminosilicate off the metallic substrate in more than one spot. The damage is shown in Figure 4.36.

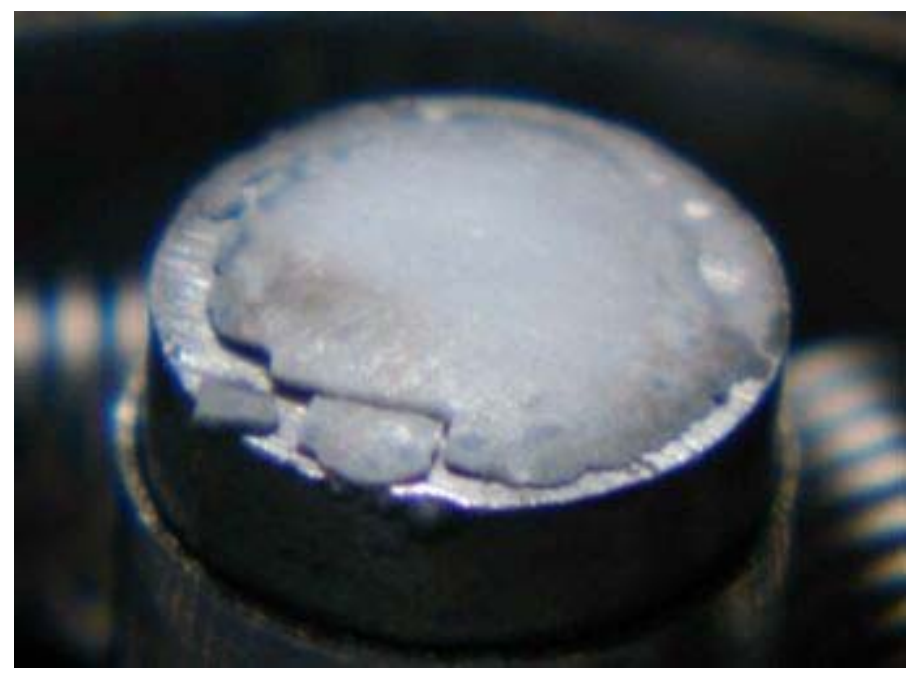

Figure 4.36. Initial damage of the K-aluminosilicate emitter upon contact with the knifeedge of the Pierce electrode.

Further application of force was able to completely separate the emitter material from the tungsten substrate. See Figure 4.37. The significance of this mode of failure 
will be revealed next. Inspection of the tungsten substrate revealed that the physical changes had not been limited to the K-aluminosilicate emitter, but also to the substrate.

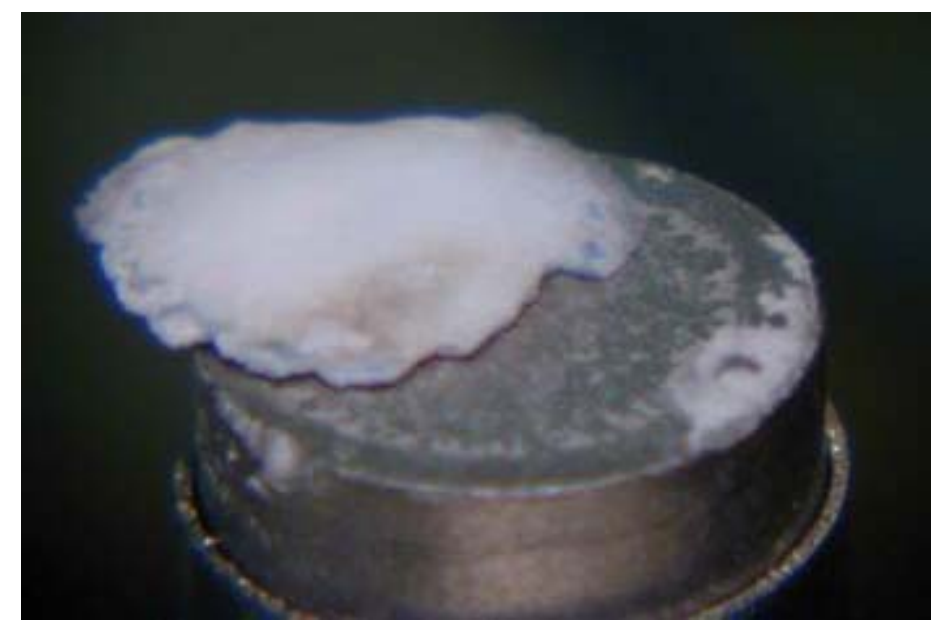

Figure 4.37 Complete separation of the K-aluminosilicate emitter from the tungsten substrate upon the application of a slight pressure.

At first sight, the tungsten substrate did not appear to have the same texture as originally. Upon closer examination of the substrate using a scanning electron microscope, the nature of the changes that the substrate underwent during the testing at high temperatures became clear. The firing induced an additional sintering of the pellet, provoking a complete closing of the pores in the surface (interface with the aluminosilicate layer) and probably throughout the bulk of the material.

Figure 4.38 shows a photograph taken with the electron microscope showing the surface with no surface porosity found after the tests. Some regions are marked by visible scratches which may presumably have been created during a sliding between the aluminosilicate layer and the tungsten metal.

Figure 4.39 shows a different region of the surface where the effects on the surface porosity are very evident. The size of the larger grains is larger than the original size of the largest grains in the original substrate. 


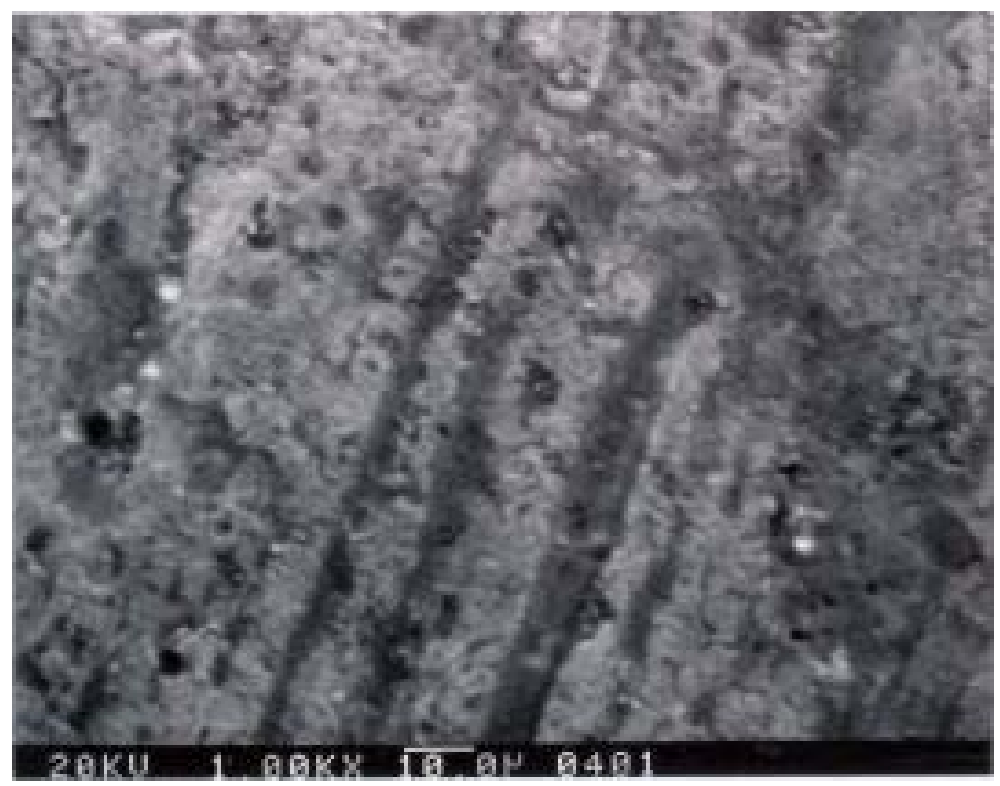

Figure 4.38. SEM photograph of the surface of the tungsten substrate, showing a surface with no porosity. The scratches are presumably the effect of a sliding between the aluminosilicate layer and the tungsten surface.

Figure 4.39 includes the image of some of the remaining material that originally belonged to the aluminosilicate. The characteristic needle-like crystal structure that appears when the aluminosilicate has been overheated is a result of significant loss of at least one of the components of the original mineral, as well as a result of component separation. In this case, as it has been discussed in Chapter 2, the main component of this crystal seems to be a form of aluminum oxide.

Figure 4.40 shows a view of the surface of the aluminosilicate that was originally in contact with the tungsten substrate. The shape of some of the protrusions suggest that such is the original shape that the aluminosilicate had upon initial melting, as the protrusions seem to match the shape of a porous material.

Two main processes may be identified in the interface region: 
1. Further sintering and grain growth of the tungsten grains that form the substrate, with a consequent reduction and final elimination of the surface and bulk porosity.

2. A change in the composition in at least a fraction of the aluminosilicate in the interface, giving rise to regions poor in potassium and silicon and rich in aluminum and oxygen, giving rise to a distinct crystal structure.

It is important to consider that when comparing the input power required to raise the temperature of the aluminosilicate surface to a certain level, it is generally necessary to provide a larger amount of input power than in the case of metallic sources. A comparison of the input powers and achieved temperatures indicates that the back of the aluminosilicate layer may be at least $150{ }^{\circ} \mathrm{C}$ hotter than the front surface.

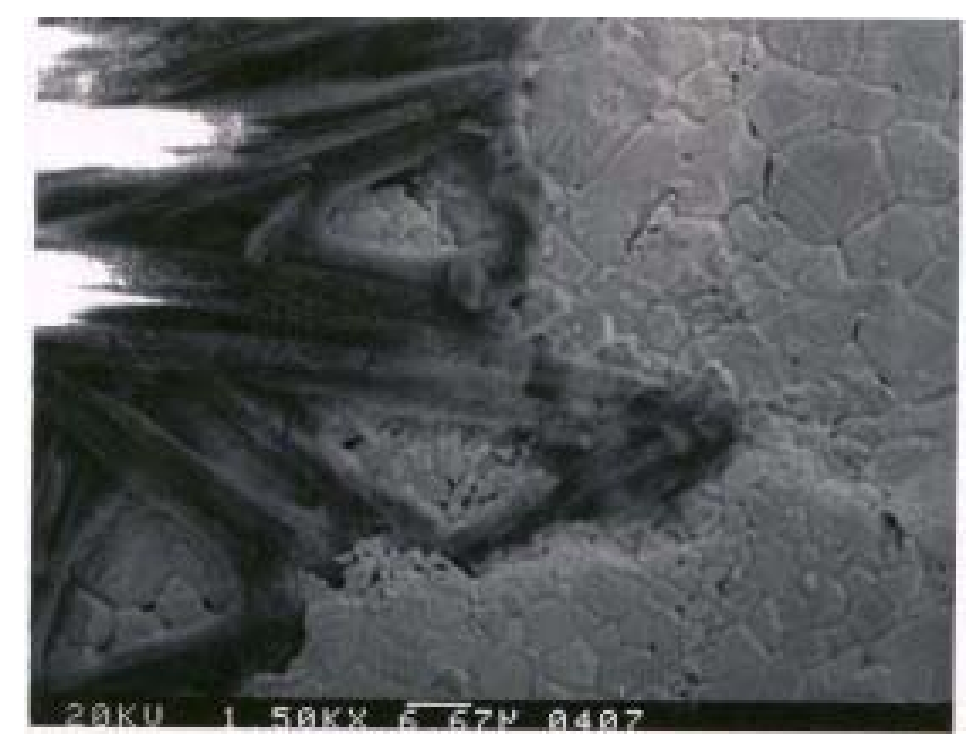

Figure 4.39. SEM photograph of a different region of the original aluminosilicatetungsten interface, showing the tungsten grain growth and subsequent pore closing. Note the crystals of aluminum oxide that have remained attached to the tungsten. 


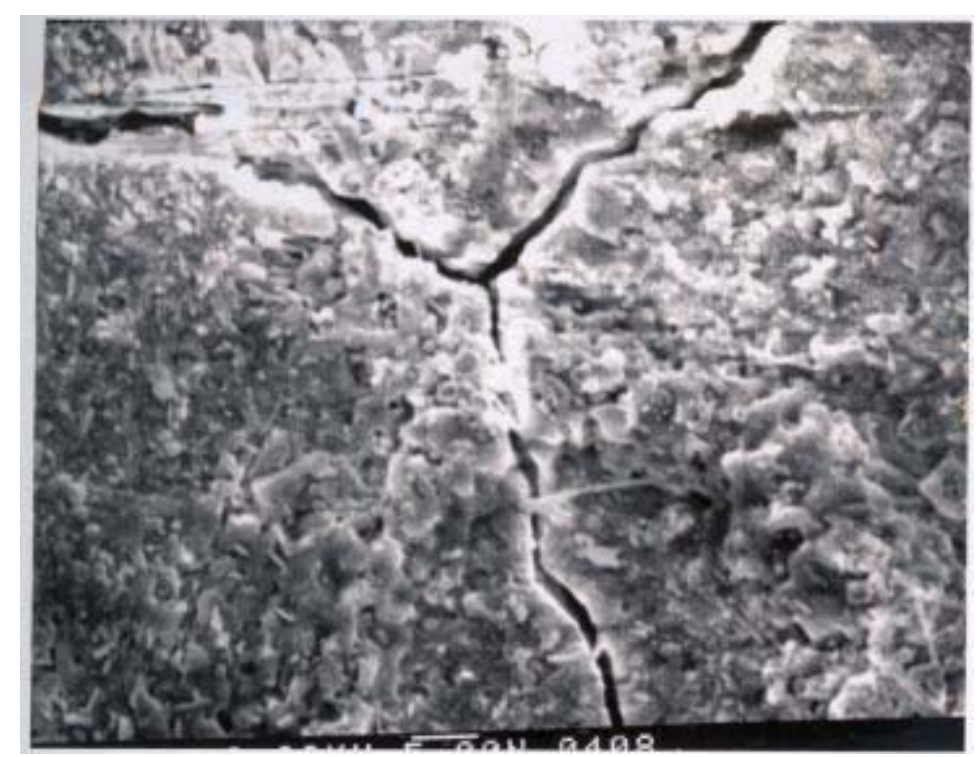

Figure 4.40 View of the back side of the aluminosilicate that separated from the tungsten substrate. The protrusions that can be observed may have been points of original contact or penetration into the tungsten substrate, before the latter began to close. The $2 \mathrm{kX}$ magnification yields a $5 \mu \mathrm{m}$ length of the reference line.

It is worthwhile to compare at this point the microscopic appearance of the aluminosilicate source before and after the test. Figure 4.41 shows a SEM photograph of the emitter used in these experiments, as it looked originally. Usually these emitters, when they have been fired correctly, become very difficult to photograph. Their surface is very smooth, glassy and as a non-conducting material, tend to charge up upon being struck by the electron beam of the microscope. The charging problems are the reason for their being hard to photograph. In some surface regions, that to the naked eye appear as slightly matte, there are usually small chunks of aluminum oxide that have separated from the molten aluminosilicate and go to the surface upon solidification. The presence of these chunks is ubiquitous whenever high emission aluminosilicate sources are considered and for some reason, they do not offer as severe charging problems as the regions of aluminosilicate free of these particles. These aluminum oxide particles are also seen in Figure 4.41. 


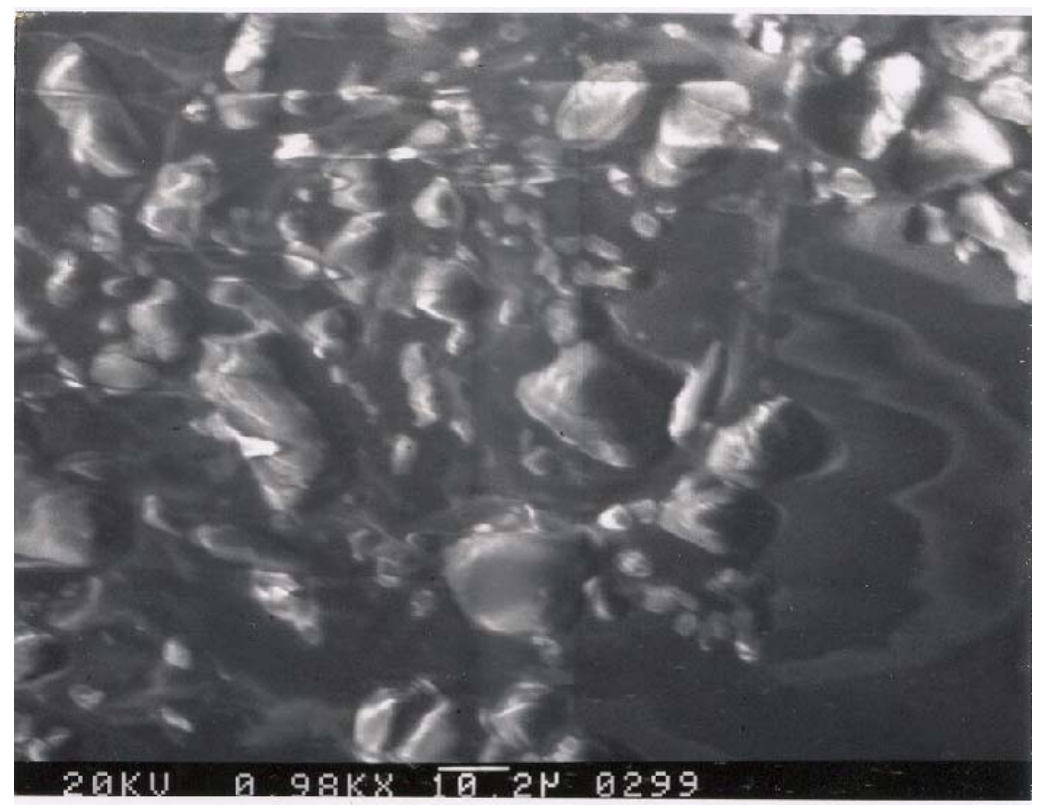

Figure 4.41 SEM photograph of the original appearance of the tested aluminosilicate emitter. The aluminosilicate is the glass-like smooth surface.

On the other hand, Figure 4.42 shows an equivalent photograph of the same ion source, after the experiments, that is, after spending more than two weeks at an operating temperature of more than $1,100{ }^{\circ} \mathrm{C}$. Since the emitter has been fully "activated" and performing at the maximum ion yield output, it is expected to see large portions of the smooth aluminosilicate surface. This expectation is validated by the image. There are nevertheless two important differences with respect to the original surface appearance. In the first place, there are no conglomerates of aluminum oxide or other type of particles in the surface, at least not in the concentrations observed before. This is consistent with the increased ion output of the emitter, since the aluminum oxide particles would presumably decrease the available area for ion emission. The second main difference is that in the tested emitter, it is now possible to see a growing matrix of needle-like crystals of aluminum oxide that was not present before, but as it has been shown, is present in the back side of the emitter layer. 


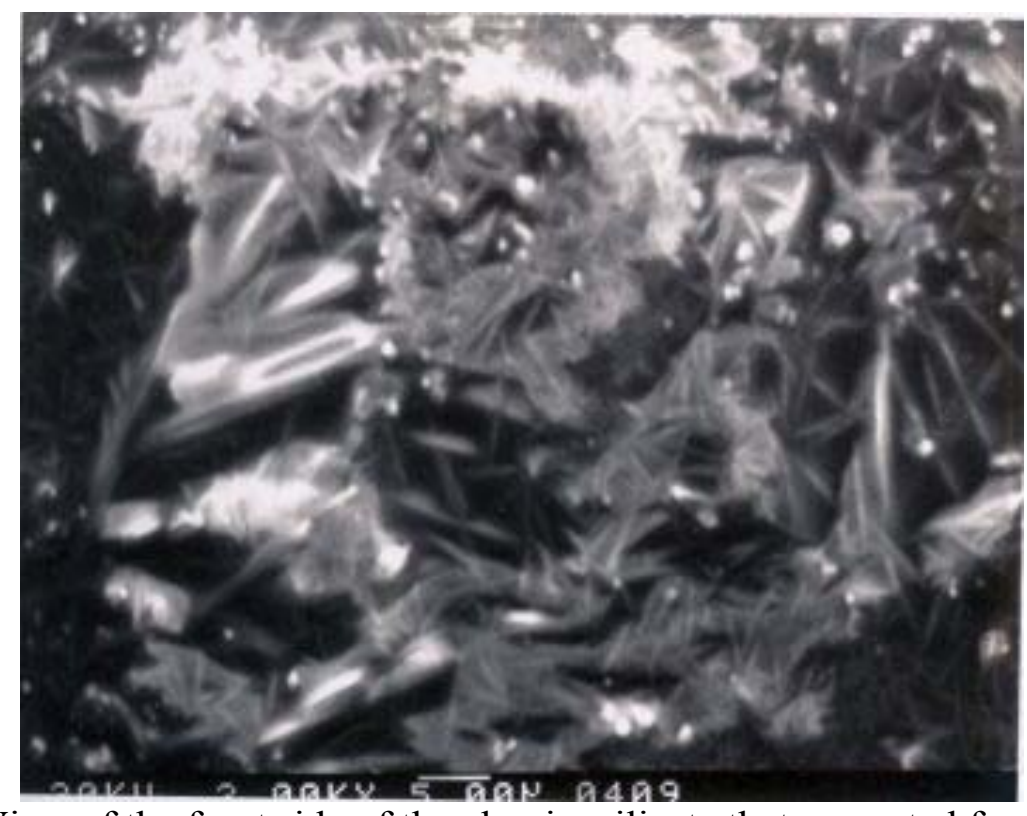

Figure 4.42 View of the front side of the aluminosilicate that separated from its substrate.

Equally revealing information is obtained when analyzing the composition of the emitter material in the front and back side of the detached layer. Figure 4.43 presents the composition spectrum of the front surface of the aluminosilicate.

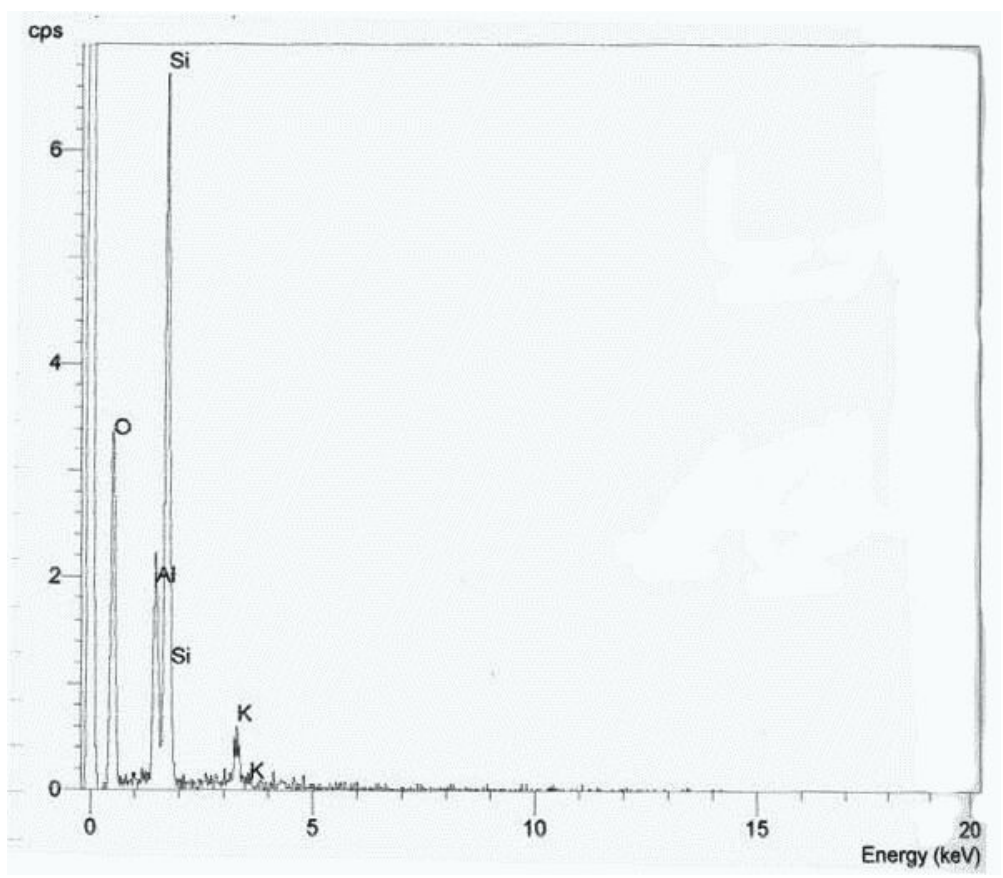

Figure 4.43 SEM spectrum of the front side of the aluminosilicate. 
The spectrum shows a typical aluminosilicate signature, with the feature of a low concentration of potassium in the surface, or at least in the first few micrometers from the surface, which is the distance that the electron beam is able to probe. The previous spectrum differs from Figure 4.44, that was taken from the back side of the emitter layer (the one in contact with the tungsten originally). There is a presence of tungsten particles that may have remained attached to the aluminosilicate, but the most characteristic feature is the total depletion of potassium.

The presence of the other aluminosilicate components may be misleading, since as it was mentioned earlier, once the structure of the substance starts revealing a needlelike structure, then there is an indication of elemental separation between the components of the aluminosilicate. Most distinctively, a separation of the silicon and the aluminum occurs, with the corresponding destruction of the aluminoslicate structure in favor of oxide forms.

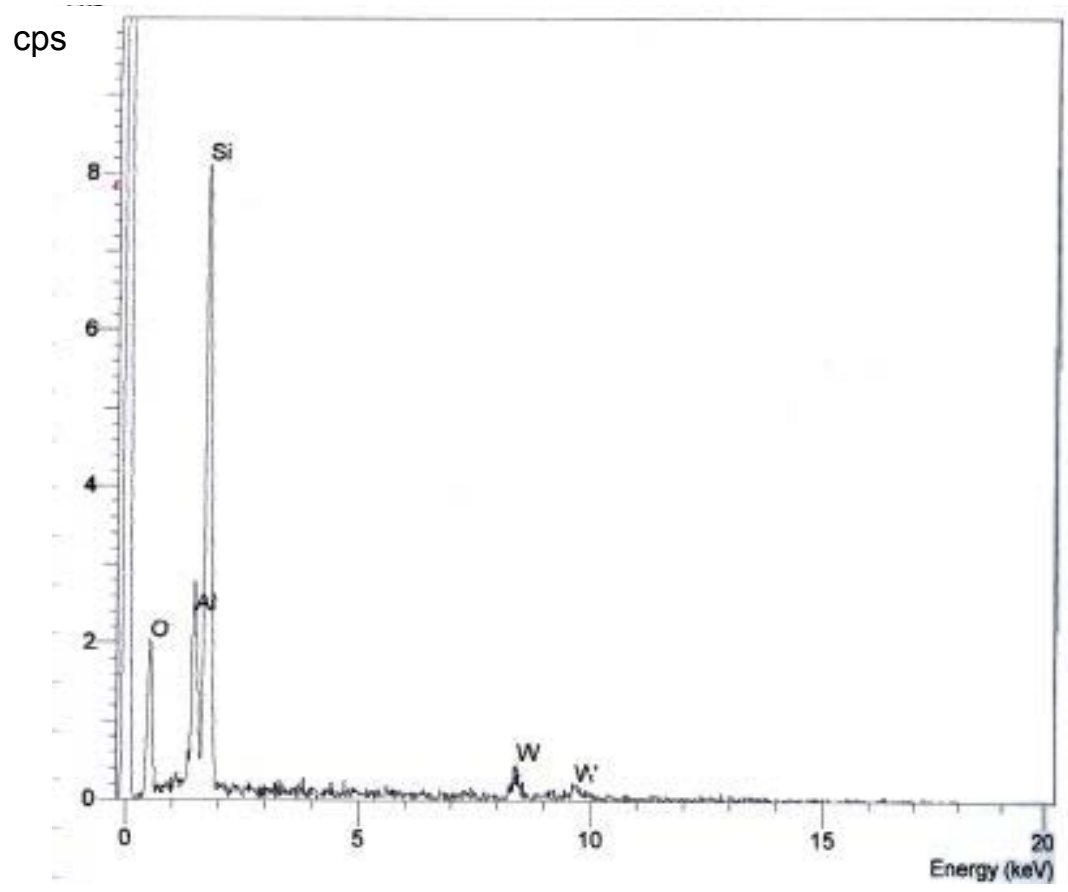

Figure 4.44 Spectrum of the back side of the aluminosilicate part. 
The aluminosilicate type of sources could never be taken to a depletion mode of failure in these experiments. Usually some other mode of failure, such as the ones described in the previous pages was observed.

\subsubsection{Evidence of recovery mechanisms in a depleted aluminosilicate source.}

The observed mode of failure of the potassium aluminosilicate sources operating in the demanding regimes described above was not related to features inherently related to the emitter. The main failure mode was related to changes in the surface condition of the substrate after long periods at high temperatures. This led directly to the question of the real lifetime of this type of source. Here the term "real lifetime" is interpreted as the lifetime that can be obtained upon extracting all the available ions in the emitter layer. This definition implies that there are possible improvements that may avoid the failure mechanisms presented above.

An accelerated depletion test was designed and carried out with the intention of measuring the available charge for extraction from an activated source. As it will be seen in the following paragraphs, the results of this test were inconclusive. Nevertheless, the method and results are presented as a reference. The main reason for including this section is to present the recovery of an emitter that was subject to this forced depletion.

To carry out this experiment, a DC high voltage power supply was incorporated in the setup, replacing the bipolar pulser used in the previous experiments. The idea of the experiment was that an application of a constant voltage would allow for a direct measurement of the emitted current vs. time. Finally, upon integration of this signal, the total charge emitted could be measured. 
For this test it was decided to use a previously activated emitter, allowing for the observation of the behavior of the source after it had been exposed to air for a period of several days.

As it will be seen, the experiment was a partial success. The effect of secondary electrons and their interaction with the beam was not taken into consideration in the new setup. In this accelerated depletion test, the negative leg of the bipolar pulser would not provide an electron repelling voltage at the extractor plane, since in this experiment, the extractor was grounded. For this reason, the secondary electrons generated at the Faraday Cup were able to stream back into the ion source. The usual electron trapping voltage was insufficient to keep all the electrons. This had two unwanted consequences. First, the current measurements have a very large uncertainty since there was an electron component to the current. The second effect was more dramatic and undesirable. The back streaming electrons at a voltage of up to $14.4 \mathrm{kV}$ provided enough heating power at the source to have a partial melting of the aluminosilicate layer. See Figure 4.45. The results, in terms of the actual charge extracted become uncertain and inconclusive, but there is a feature that justifies the inclusion of this section. That is the recovery of the emitting capability of the aluminosilicate (or what was left of it) after depletion. 


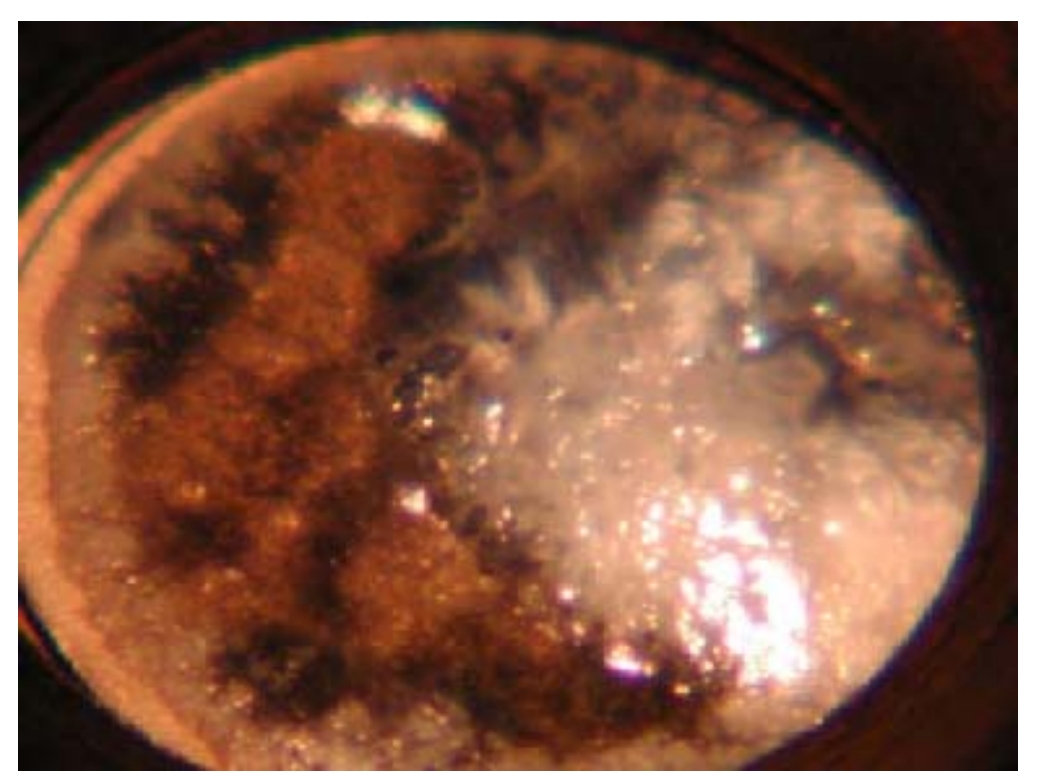

Figure 4.45. Partially destroyed aluminosilicate layer due to the action of backstreaming electrons in a 14 minute DC test.

The history of the test is summarized in Figure 4.46. The test lasted a very short time, roughly 14 minutes. Three extraction voltage levels were applied to the source: 4.2 , 9.8 and $14.4 \mathrm{kV}$, after which no current was detected. As mentioned before, since the total current measured had an electron component, it was necessary to make some assumptions in order to at least approximate the actual number of ions extracted. It has been measured that the secondary electron coefficient of $25 \mathrm{kV} \mathrm{K}+$ ions on steel is 6.5 . It was assumed that the working number for the secondary electrons for this experiment should be at most 3. Furthermore, assuming that $100 \%$ of these electrons make it back to the emitter, then only $25 \%$ of the total current measured would be ascribed to ions. Upon integration of the curve represented by the data points in Figure 4.46, the total charge ascribable to ions in this experiment was 0.21 Coulombs, or $1.31 \cdot 10^{18}$ ions. This is possibly a conservative estimate. For reference purposes, if such were the actual ion delivery capability for this source, it would be able to provide $8.3 \cdot 10^{6}$ pulses, assuming 
the pulses are $10 \mu \mathrm{s}$, and $\mathrm{J}=90 \mathrm{~mA} / \mathrm{cm}^{2}$. A production ion source working at $10 \mathrm{~Hz}$ would deplete this supply in about 10 days.

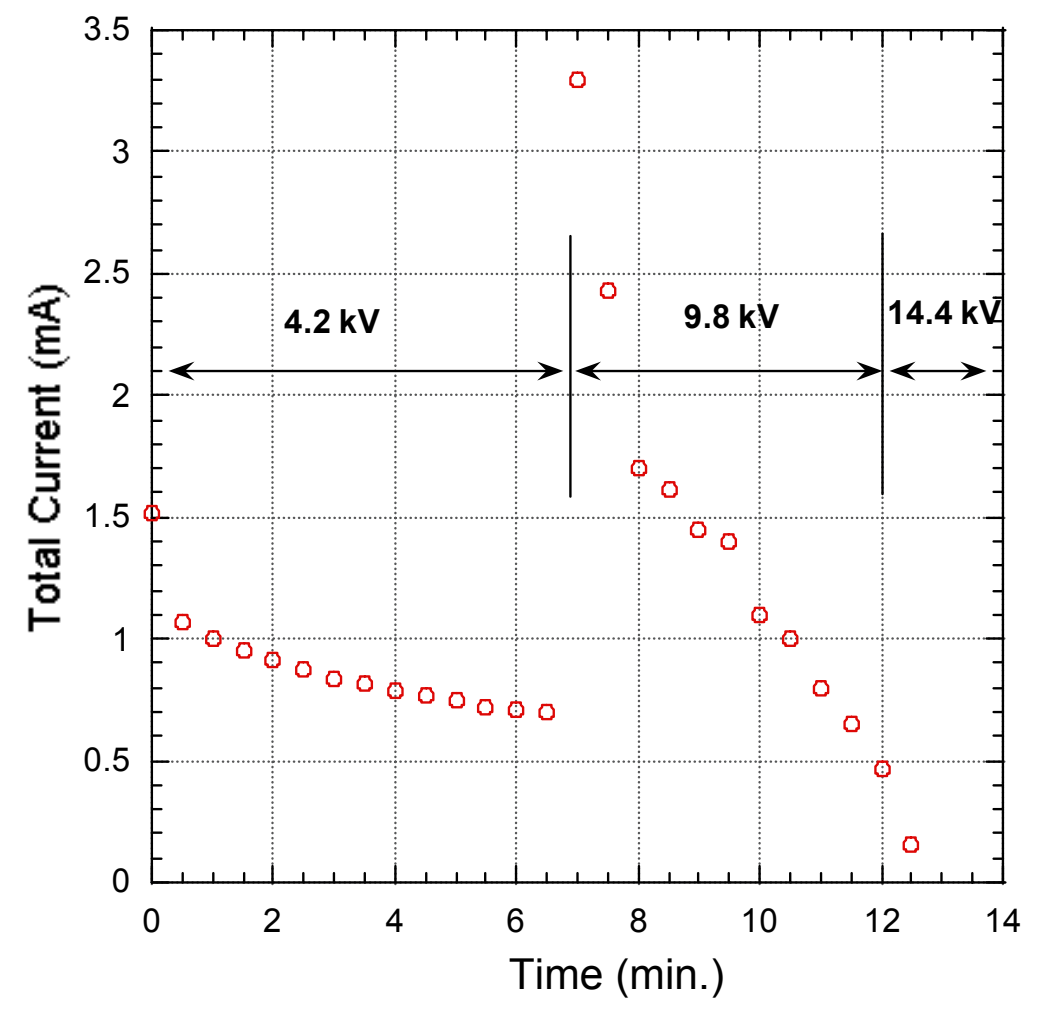

Figure 4.46 Summary of the DC forced extraction test for depletion of an aluminosilicate source. Note: total current has an electron component and must be adjusted to estimate the total number of ions delivered.

At this point it is useful to introduce some features observed in the preparation of this source test. An important finding is that if a source has been previously activated and subsequently exposed to air for a period of less than a month, it will require another activation period upon installation. Figure 4.47 shows a summary of the obtained results. A problem in the data acquisition system provoked the loss of information for a portion of the test. Nevertheless, the available data gives a suggestive picture of what was happening. The period of recovery for the source was comparable to what was seen in 
Figure 4.31 despite the fact that lower operating temperatures were used. It is nevertheless a fact that the performance of the source is affected by its exposure to air, thus requiring further activation.

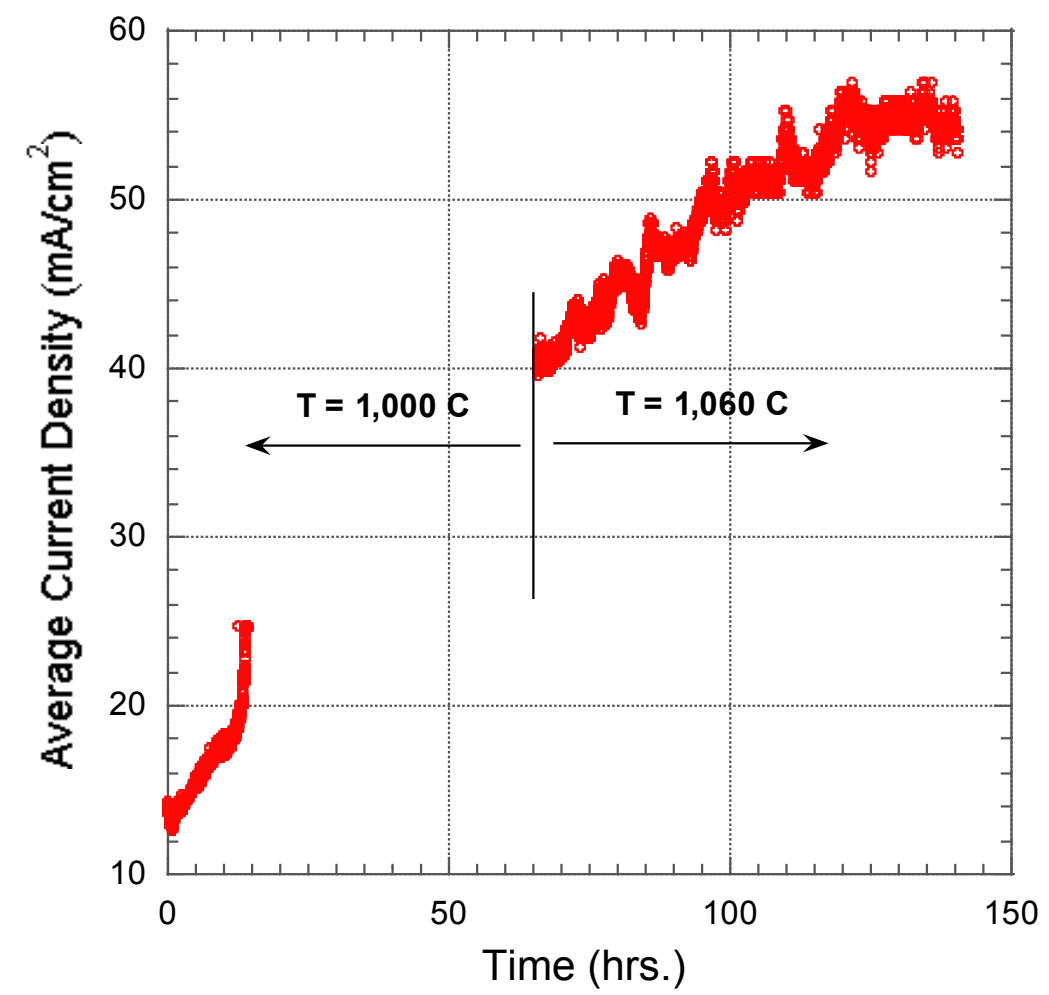

Figure 4.47. Second activation of a K aluminosilicate emitter. A problem in the data acquisition system provoked a loss of a fraction of the data. Nevertheless, the information is sufficiently revealing as to the source performance. In contrast with Figure 4.31, the activation occurs in a similar period of time but lower temperatures are required.

After the depletion (destruction!) of the source was completed and no DC current level was being measured in the oscilloscope, the source was kept at an operating temperature while the DC extraction power supply was changed back to a pulser. Once this was completed, the source output was logged in a pulsed mode. The surprising results are presented in Figure 4.48. 


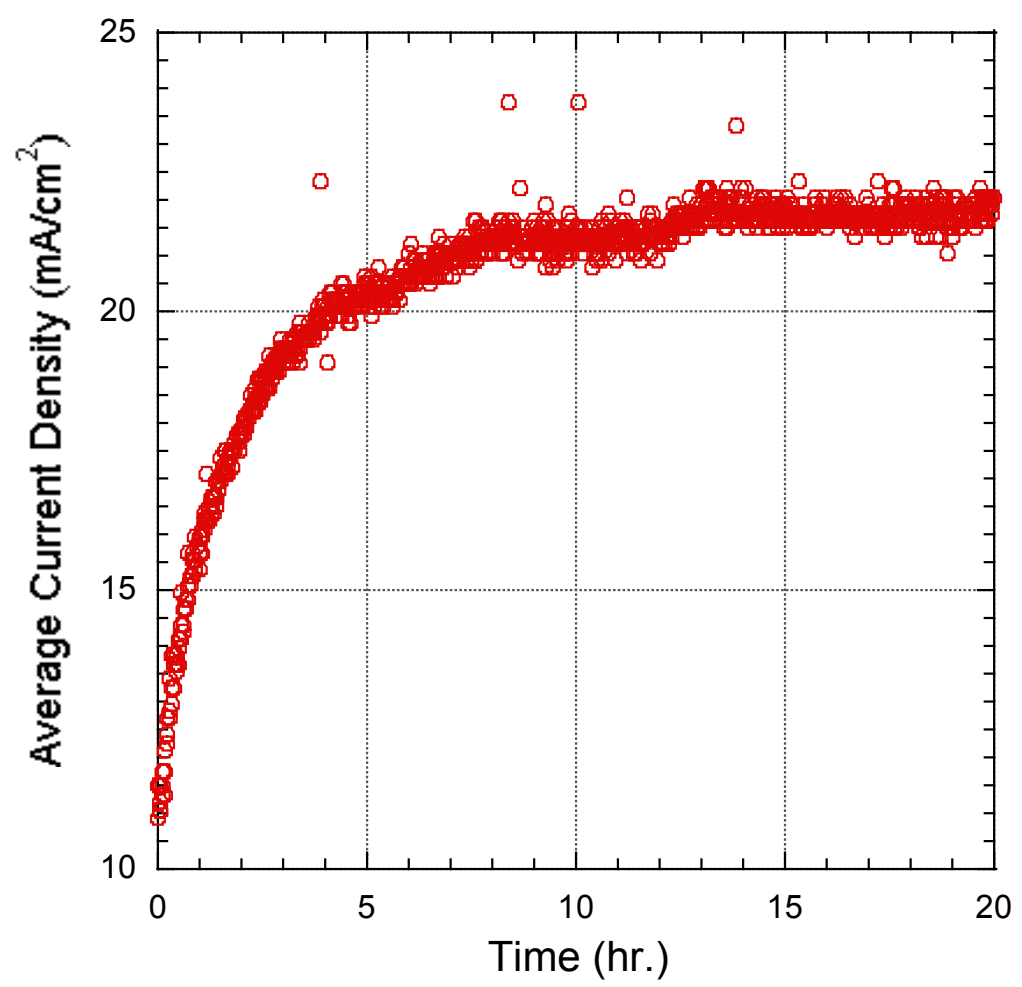

Figure 4.48 Recovery of an aluminosilicate source after a forced depletion with a DC voltage. The current values have been averaged over the original area of the emitter, but it must be remembered that the actual emitting area was only a fraction of the original.

The rapid increase in current indicates the existence of a recovery mechanism in the depleted aluminosilicate layer that allows for an increased current emission. Two main mechanisms are conceivable in this situation. One possibility is that the depleted layer is eliminated by evaporation. This would allow for the appearance of new aluminosilicate material with sufficient potassium for ion extraction. This mechanism does not seem to be the most likely. The reason for this is that in all previous cases studied, while there was an indication of small material losses from the outermost layers of the aluminosilicate into the environment, the process occurred in a time scale of days, not hours. 
It seems more likely that the aluminosilicate, and/or the phase that was left after the potassium was extracted, permits the diffusion of at least one species, in this case $\mathrm{K}$, replenishing the outermost layers and thus allowing for some level of ion extraction.

When comparing the $\mathrm{J}-\mathrm{V}$ characteristics of the emitter before and after the depletion test, we arrive at the data sets presented in Figure 4.49.

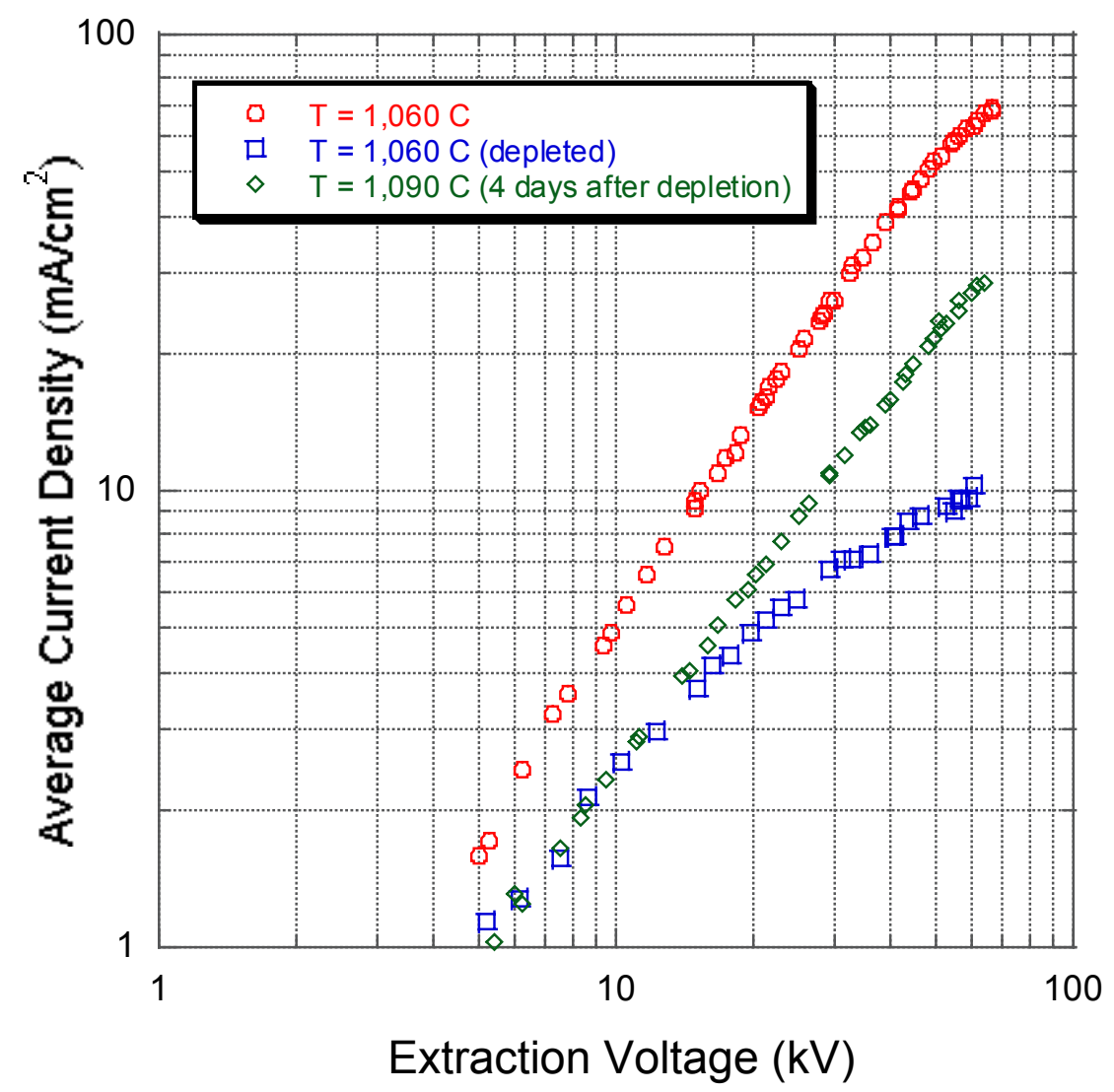

Figure 4.49 Comparison of $\mathrm{J}-\mathrm{V}$ characteristics before and after depletion of the aluminosilicate emitter. The "depleted" curve was generated within $1 \mathrm{hr}$ of the depletion.

The curve with the highest current density represents the performance of the source immediately before the test, with the characteristic $\mathrm{V}^{3 / 2}$ behavior. It came as a surprise that within a short time after the test was made (actually the time it took to 
substitute the DC power supply by the pulser, or about 1 hour), the emitter was capable of delivering up to $10 \mathrm{~mA} / \mathrm{cm}^{2}$, in $2.5 \mu$ s pulses. The fully "regenerated" output of the emitter after depletion does not quite follow a $\mathrm{V}^{3 / 2}$ behavior, as it can be seen from Figure 4.50. In this figure, the ratio of the $\mathrm{J}-\mathrm{V}$ characteristics of the emitter "after" and "before" the test is presented. This ratio also provides a guide to the ratio of the emitting area of the damaged emitter versus the original area, assuming that the ion delivery capability of the ion source per unit area before and after the test is equal at low current densities. This would give an emitting area of $54 \%$ of the original emitter, which seems believable when looking at Figure 4.45.

After finishing this test, it was important to observe the microscopic characteristics and the composition of this aluminosilicate. Inspection in the scanning electron microscope showed a common glassy surface, impossible to photograph due to the usual charging problems associated with these materials. The elemental composition identified is presented in Figure 4.51. The spectrum shows an extremely small, yet detectable, potassium signal. This falls into the pattern that the high yield aluminosilicate emitters have low potassium content. 


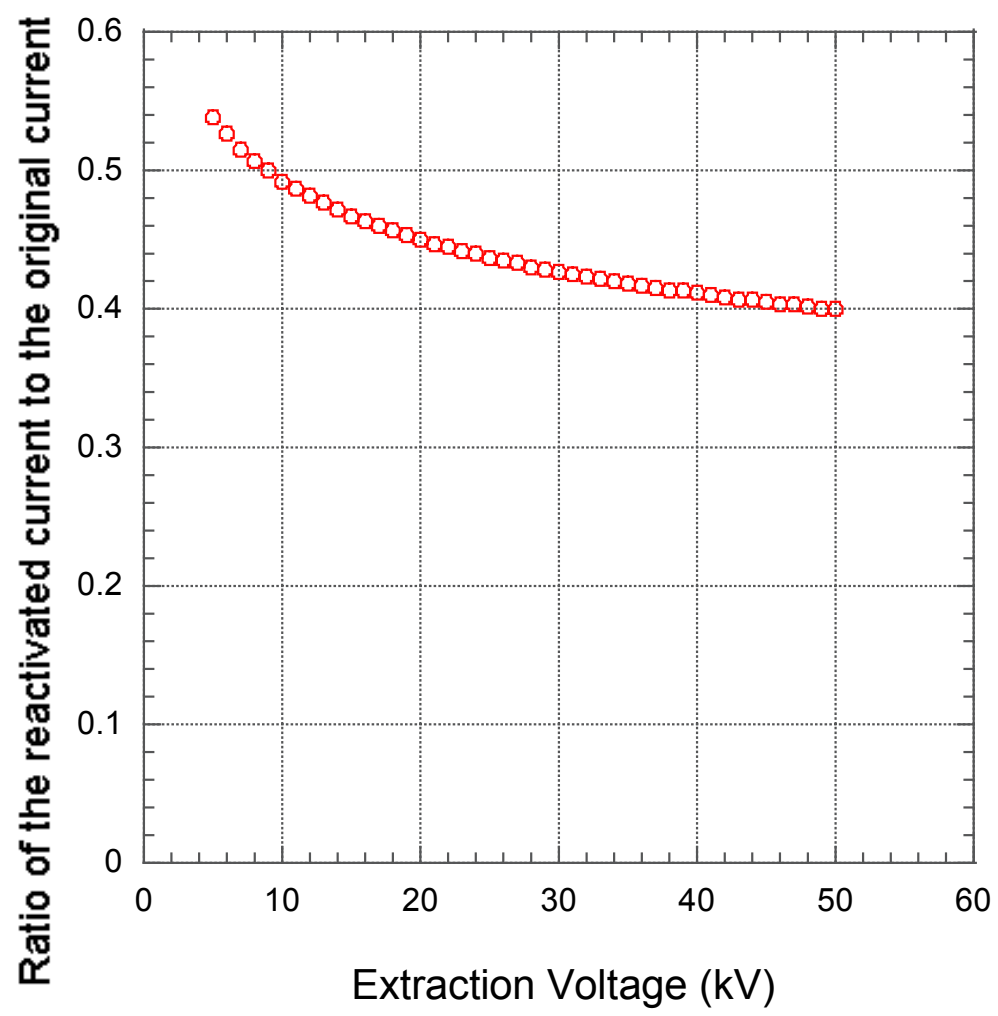

Figure 4.50. Ratio of the current of the reactivated emitter after depletion to the original current previous to the depletion.

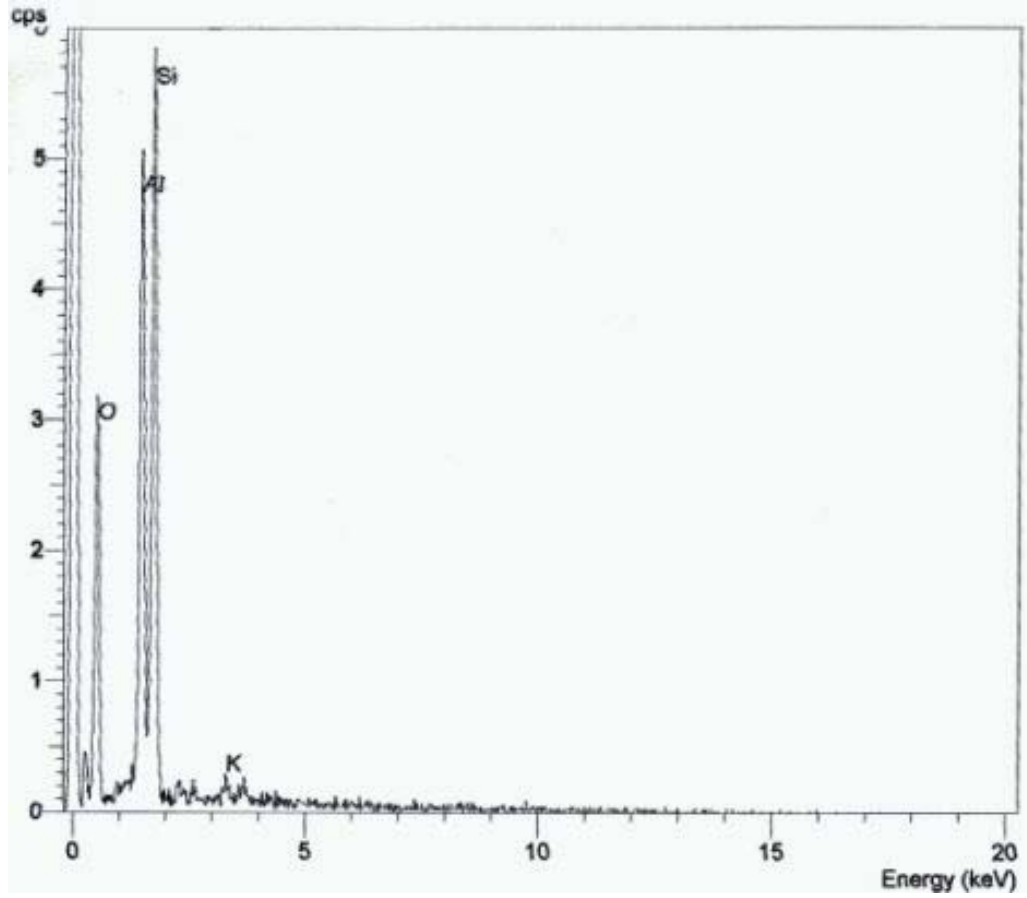

Figure 4.51. X-Ray spectrum of the depleted aluminosilicate pellet after re-activation. 


\subsubsection{Composite Material Performance}

Among the different ion source materials tested, there has been interest in composite materials. These would presumably combine the advantages of aluminosilicate sources, like better emission stability and longer lifetime, with the advantages of metallic substrates, like ease of forming, and electrical conductivity. While some of the composites tested could be eligible for production sources requiring current density of a few $\mathrm{mA} / \mathrm{cm}^{2}$, none showed promise for high $\mathrm{J}$ operation. As an example, note the performance of a molybdenum/potassium aluminosilicate composite pellet depicted in Figure 4.52. The emitter was a cylindrical pellet of approximately $1 / 4$ " diameter and $1 / 4$ " length prepared by mixing ordinary potassium aluminosilicate powder with molybdenum powder of $6 \mu \mathrm{m}$ nominal size, pressed and sintered at $1,500{ }^{\circ} \mathrm{C}$. The microscopic appearance of its surface is shown in Figures 4.53 and 4.54. A feature of the composite pellet is that its composition can be varied in the longitudinal direction. The one depicted in Figure 4.53 is made out of a 2:1, Mo:KalSilicate composition ratio by weight on its front surface (front half of the pellet), and a 3:1 ratio in the back, to enhance heat conductivity. In general, in any composite material there is a decrease of the heat conductivity in the bulk of the material with respect to a pure metal due to the presence of the aluminosilicate. This is clear from the increase in input heating power required to raise the surface temperature, compared to porous metallic substrates and to pure aluminosilicate layers deposited on pure metal. Figure 4.52 shows the $\mathrm{J}-\mathrm{V}$ characteristics for this composite at different temperatures. It is evident from the curves that the space charge limit of the source operation is achieved in the lower range of the applied voltages. It was necessary to apply the maximum power available at the 
experiment (approximately $240 \mathrm{~W}$ ) to attain an operating temperature of $1,230{ }^{\circ} \mathrm{C}$, at which the source could barely deliver $5 \mathrm{~mA} / \mathrm{cm}^{2}$ of potassium in a space charge limited mode. For this reason, such composites could be eligible for making thin layers on top of metallic substrates in situations where such current densities may be required.

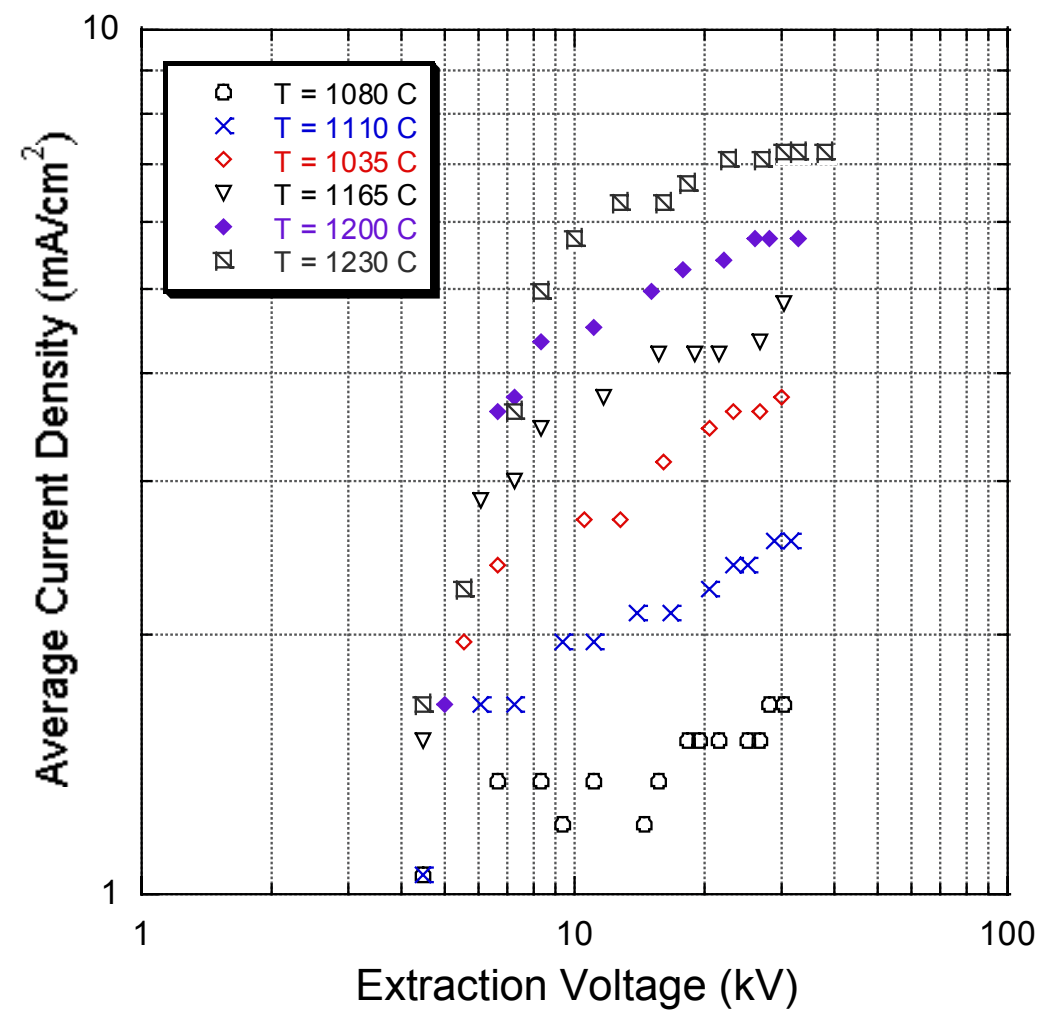

Figure 4.52. Performance at different operating temperatures of a Mo-K-aluminosilicate composite.

The data displayed on the previous graph was taken after 21 hours of continual operation at a temperature of $1,080{ }^{\circ} \mathrm{C}$, thus allowing for a possible "activation" of the aluminosilicate component. Despite a slight improvement in the output, such activation was not observed. Figures 4.52 and 4.53 show an example of the distribution of molybdenum and aluminosilicate grains on the surface. Undesired non-uniformities on this distribution can be seen with respect to central and outer regions. The photographs were taken with a scanning electron microscope at 1,000 times magnification. The mode 
chosen for the images is the backscattered electron detection mode which allows for a differentiation of the conducting and non-conducting components of the mixture. The molybdenum component can be easily appreciated due to its bright coloring.

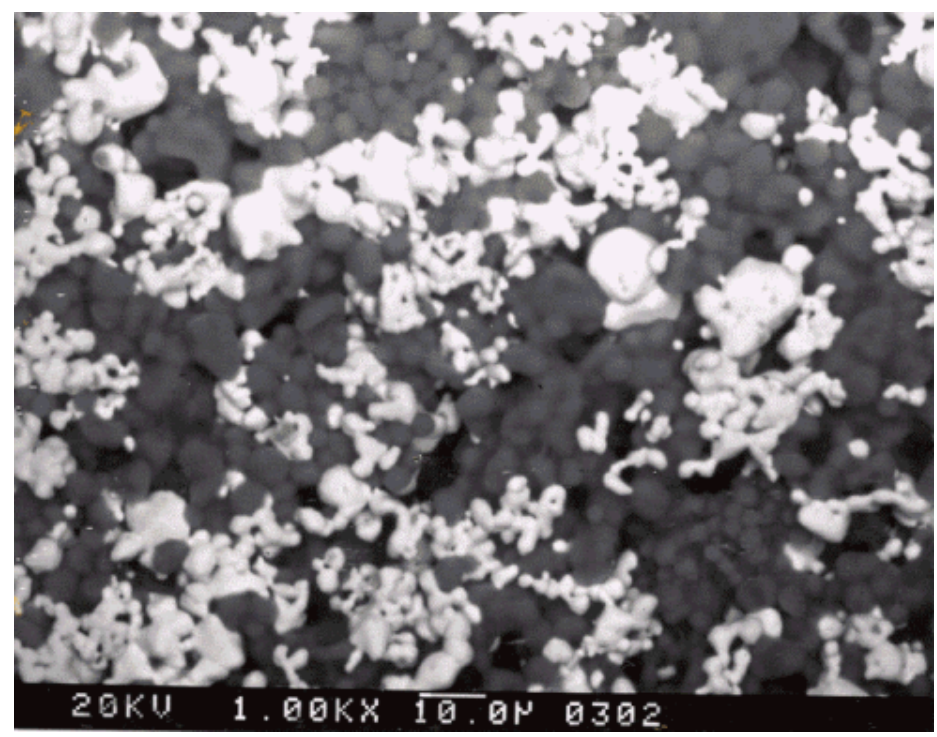

Figure 4.53. Backscattered electron photograph of the surface of a molybdenum-K aluminosilicate composite pellet near its center. Brighter grains correspond to Mo. Note the wide distribution of Mo grain sizes around the nominal size of $6 \mu \mathrm{m}$.

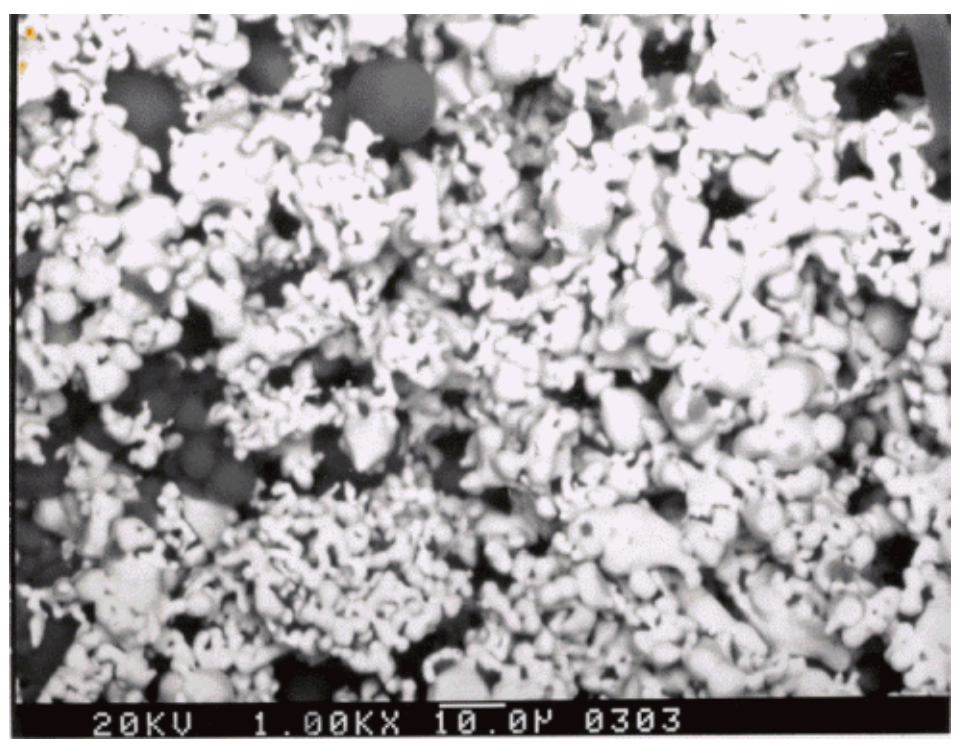

Figure 4.54. Backscattered electron photograph of the surface of a molybdenum-K aluminosilicate composite pellet, near the edge. Note the increased Mo content. 
Analogous tests were carried out using a different metallic component. Figure 4.55 shows the $<\mathrm{J}>$ vs time operation characteristics of a potassium aluminosilicate / tungsten powder mixture. This pellet had the same geometric characteristics as well as similar composition properties as the previous Mo composite mentioned above. Figure 4.55 shows the rapid response to a temperature increase of the current output (note the discontinuities in the output curves at different temperatures), as well as the relatively rapid decay in this parameter with time. The decay of output current in time scales of a few hours seems to be a characteristic of composite materials and can be understood in terms of the apparent ability of the alkali ions to migrate within the aluminosilicate structure. In interconnected aluminosilicate structures, this migration or diffusion capability may provide a relatively sustained concentration of atoms at the surface, capable of delivering sustained current levels typically observed in aluminosilicates.

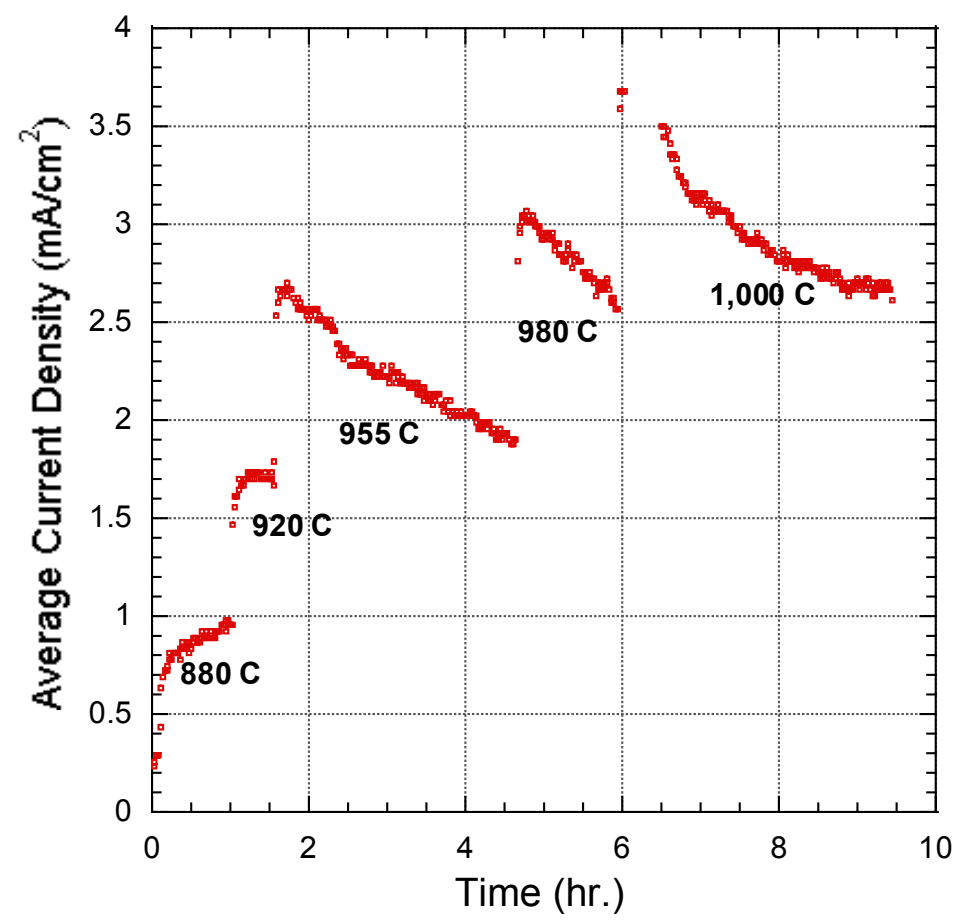

Figure 4.55 Life test of a potassium aluminosilicate/tungsten composite pellet. 
The lack of interconnectivity of the aluminosilicate structure present in the composite materials (note the globularized microscopic structure seen in Figures 4.53 and 4.54), will presumably diminish the reservoir of potassium atoms for the available emitting surface, thus allowing for a relatively rapid decrease in the ouput current. Figure 4.56 shows the $\mathrm{J}-\mathrm{V}$ characteristic at the final operating temperature of $1,000{ }^{\circ} \mathrm{C}$. As in the Mo/aluminosilicate composite, the performance in the space charge limited mode was limited to a few $\mathrm{mA} / \mathrm{cm}^{2}$. It is noteworthy, however, that the performance tests with this particular W/aluminosilicate composite were done with a long pulse high voltage extraction machine, and that the composite has thus demonstrated a capability to yield pulse lengths of up to $10 \mu \mathrm{s}$.

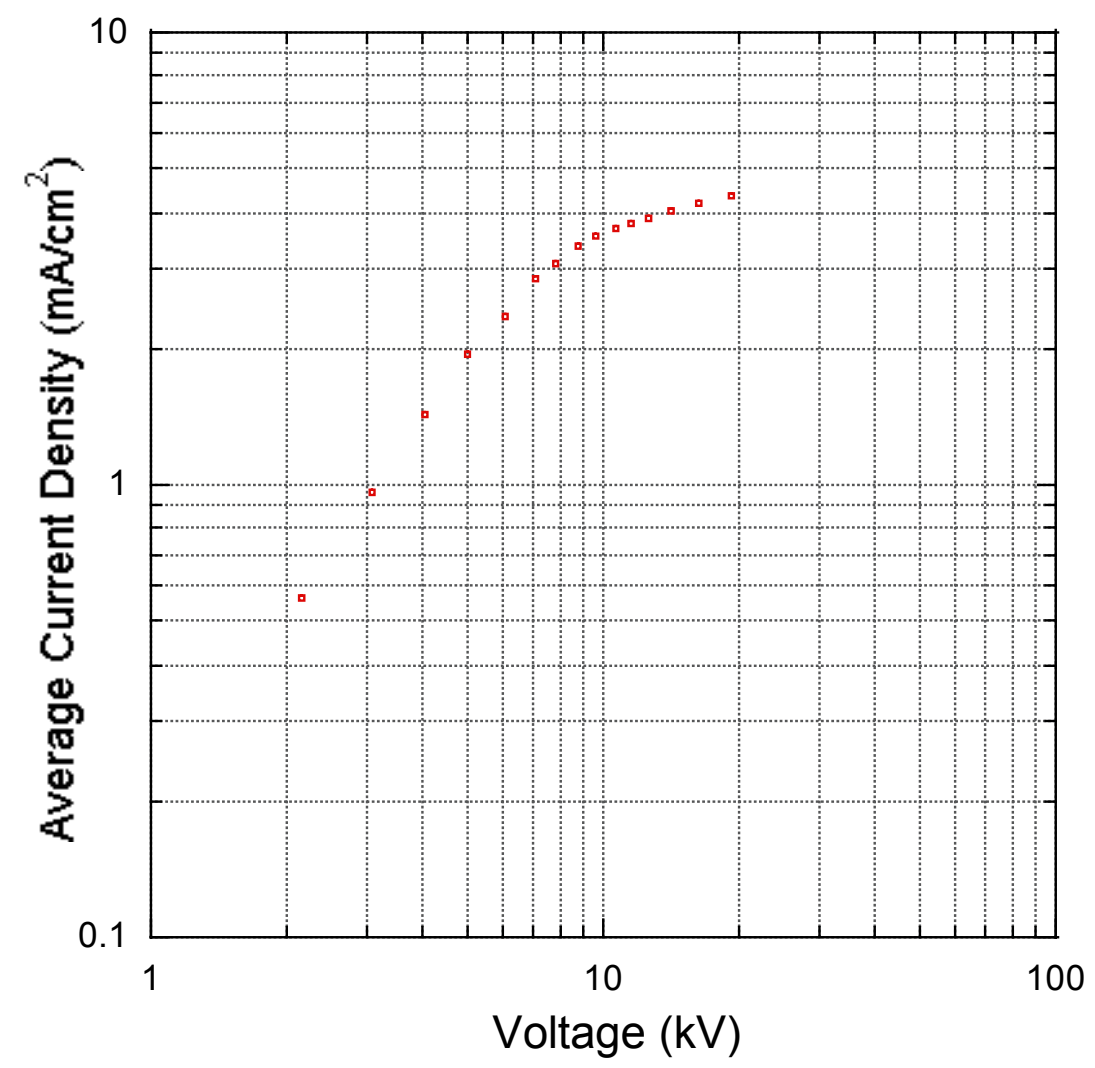

Figure 4.56 $\mathrm{J}-\mathrm{V}$ characteristic of a tungsten/K aluminosilicate composite, operating at a temperature of $1,000^{\circ} \mathrm{C}$. 


\subsection{Neutral emission Measurements.}

The theory of neutral particle emission from tungsten surfaces has been elaborated elsewhere (Langmuir, 1933). However, there has been a need for direct measurement of this process in HIF research given the diversity of non-ideal conditions of the sources under consideration (diverse degrees of surface porosity, contaminants, unknown surface coverages, low duty factors of beam time, etc.). This section presents the general results obtained with this device, which in turn has been discussed in Section 3.3.2.

\subsubsection{Neutral Particle Detector Design}

The essential features of this device are shown in Fig 3.8. The detector works as follows: an incoming neutral efflux from an ion source enters the detector and strikes an incandescent (ohmic-heated) platinum foil. The foil adsorbs the atoms and due to its high electron work function of $5.39 \mathrm{eV}$, will ionize them efficiently. A negatively biased collector produces a constant electric field on the platinum foil, extracting the newly formed ions and collecting them. The casing of this setup has been biased positively to prevent the ions from striking the casing or leaving the detector. Grids of $90 \%$ transparency are located in the casing and collector apertures to ease the collection of particles, aid in shielding and improve the field uniformity.

Ions arriving at the collector produce a very small current that is measured through the voltage drop across a large resistance on its path to ground. A $10 \mathrm{G} \Omega$ input impedance multimeter is used for this purpose. Another voltage monitor was used to 
detect any current arriving or leaving the casing. If any, such currents were too small to be detected.

The conversion factor that translates currents measured at the neutral detector to neutral particle density emitted at the source is determined by the geometric relation between the emitter and the platinum foil, and is estimated in the same way as the "shape factors" common in radiation heat transfer. See section 3.3.2.

\subsubsection{Neutral Measurement Results and Discussion}

The neutral detector was used to monitor the particle efflux from $\mathrm{K}$ and $\mathrm{Cs}$ sources on porous tungsten substrates during the source warming up cycle, as well as at operating temperatures.

During the heating-up cycle, most of the phenomena that occur within the source have to do with the decomposition of the alkali carbonate that has been used to dope the source, its distribution in the bulk and the evaporation of the alkali atoms as neutral particles. During all this time, no ion desorption is detectable. The neutral detector thus has become the only means to assess the source phenomena before the ion yield is detectable. These phenomena had not been assessed previously for a source of this type.

Curves such as the one shown in Fig. 4.57 were obtained. These curves qualitatively display the dynamical character of the neutral evaporation processes, with increasing rates at increasing temperatures, a corresponding decrease in neutral atom content with time, and the effects of enhanced diffusion of the alkali atoms in the tungsten bulk with temperature. At any given moment, the neutral flux is calculated from 
the difference of the values of the signal in the line of sight of the source and the background signal, which is checked periodically. The signal at the detector when it is out of the line of sight of the ion source is not zero, probably as it is still able to detect the bombardment of alkali particles present in the vacuum tank's residual atmosphere.

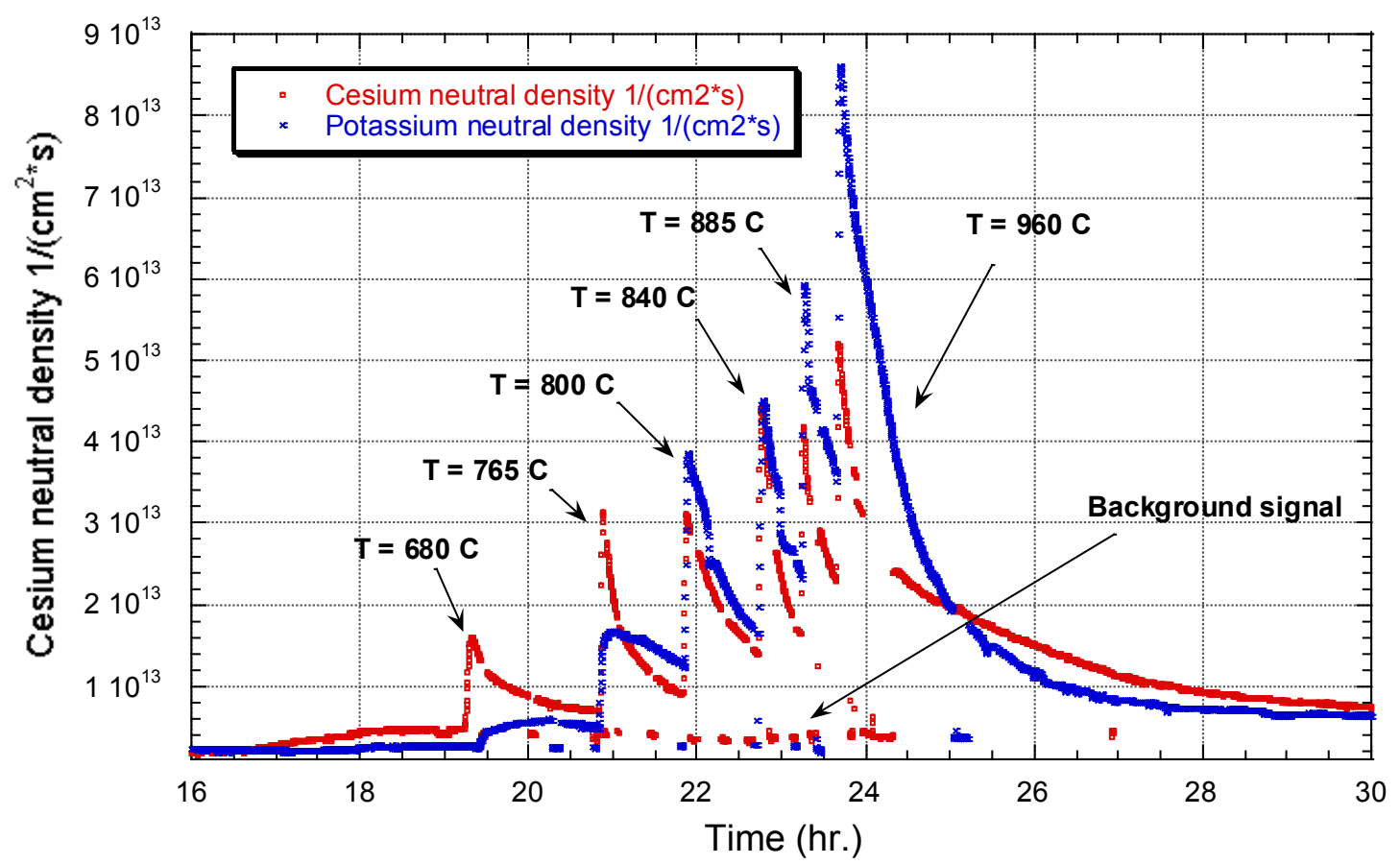

Figure 4.57. Sample data set of a heating cycle of Cs and K contact ionization sources measured with the neutral detector.

The above data set shown in Figure 4.57 was measured by positioning the neutral particle detector directly in the line of sight of the ion source. The conversion factor that relates the measured currents at the detector with the neutral effluxes at the emitter has been calculated for this position. The signal at the detector is very distinct and the sensitivity of this instrument detects very small variations in the incoming neutral flux. Figure 4.58 shows a sample data set obtained by changing the position of the neutral detector relative to the emitter, in a direction perpendicular to the beamline. The curves 
have the expected shape and width given the system geometry. In the particular set of data shown in the figure, there is no regular trend in terms of the neutral output variations in temperature as the time-dependence effects shown in Figure 4.56 have not been included.

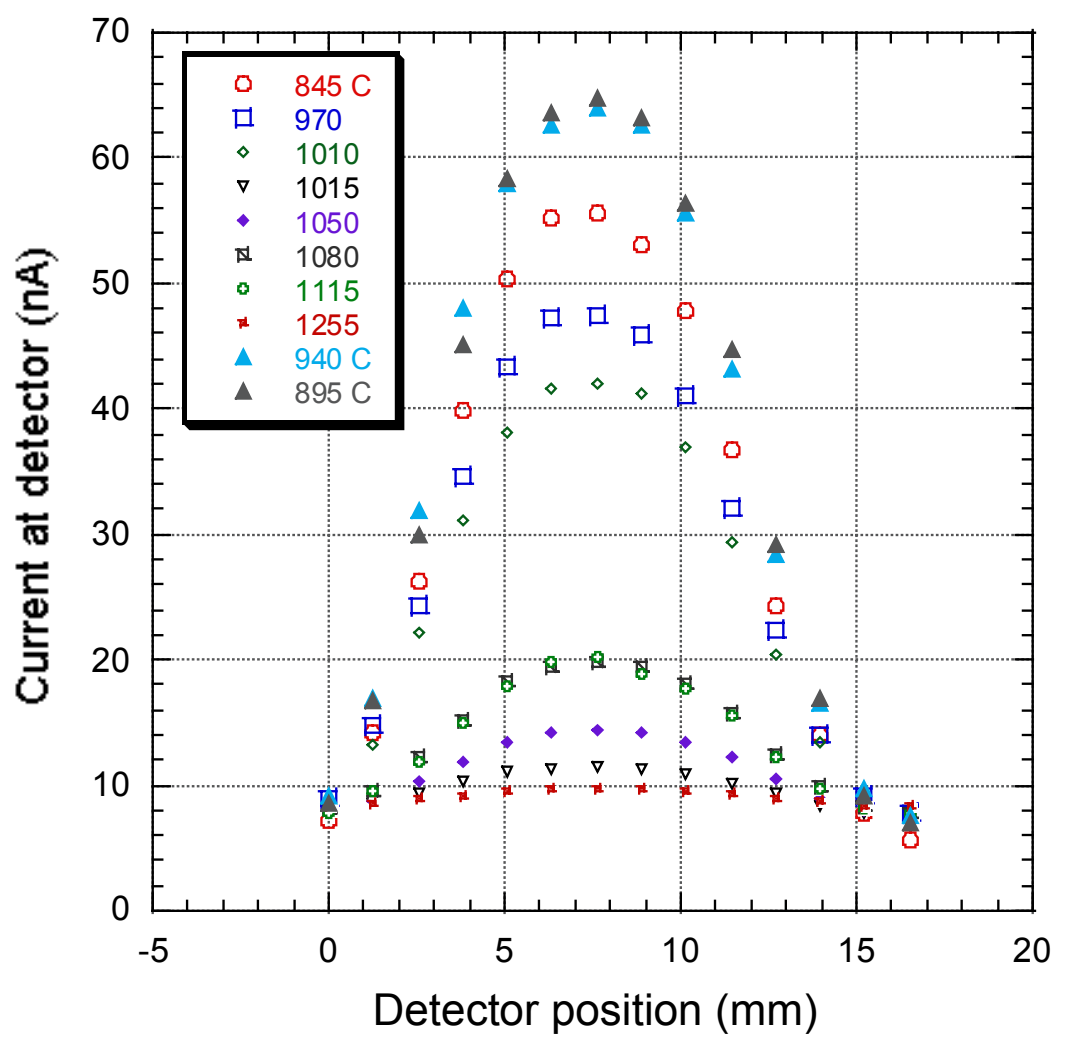

Figure 4.58. Sample data representing the signal at the neutral detector as a function of position relative to the emitter. Data such as in Figure 4.58 were measured at the central position, directly in front of the ion source.

Neutral emission in doped sources at low alkali coverages and operating temperatures (T approximately $1,100{ }^{\circ} \mathrm{C}$ ) yielded values in the neighborhood of $0.3-0.5$ $\mu \mathrm{A} / \mathrm{cm}^{2}$ of current equivalent with a tendency to decrease with time. In general, potassium neutral emission was observed to be higher by a factor of 1.5 to 2 at similar points of the heating cycle except at the low temperatures where the carbonate is expected to break down. Previous measurements of neutral emission efflux (Kwan, 
1997) carried out at LBNL made on porous tungsten using front feeding of pure Cs vapor estimated particle densities of $1.7 * 10^{14}$ particles $/\left(\mathrm{cm}^{2} * \mathrm{~s}\right)\left(\sim 27.2 \mu \mathrm{A} / \mathrm{cm}^{2}\right)$ in current equivalent) at an operating temperature of $1,100{ }^{\circ} \mathrm{C}$. Such rates of neutral particle loss seemed inconsistent with measured lifetimes of more than $100 \mathrm{hr}$ of a Cs doped source yielding current densities above $10 \mathrm{~mA} / \mathrm{cm}^{2}$. Since the long lifetimes were measured on sources doped with an alkali carbonate solution, two possibilities were identified: it is believed that the decomposition of the carbonate leaves behind an absorbed layer of oxygen that increases the tungsten work function $(\phi)$, thus decreasing the neutral efflux. Oxygenated tungsten has a higher $\phi$ than clean tungsten, with a value $\sim 5 \mathrm{eV}$ (Cho, 1964). Such value would decrease the neutral population of atoms on the surface, decreasing their loss rate. Clean tungsten surfaces are sensitive to adsorbed oxygen caused by pressures as low as $1 * 10^{-8}$ torr (Husmann, 1966), so some effect is expected as our base pressure is $\sim 5^{*} 10^{-7}$ torr. In this experiment, typical emission rates of neutral particles at operating temperatures $\left(\sim 1,100{ }^{\circ} \mathrm{C}\right)$ were on the order of $0.3-0.4 \mu \mathrm{A} / \mathrm{cm}^{2}$ of $\mathrm{J}$ equivalent $\left(1.875 * 10^{12}-2.5 * 10^{12}\right.$ particles $\left./ \mathrm{cm}^{2} * \mathrm{~s}\right)$. This would signify tens of hours of operation before depletion even at an initial total alkali coverage of $30 \%$ (that is, including internal areas). This is a result fully consistent with the observed lifetimes of sources doped with an alkali carbonate solution. The other possibility has to do with the diffusion rates of the ions and neutrals within the bulk, that may be the limiting factor for the rate of arrival of neutrals at the surface. This will be discussed in detail in Chapter 5 .

In general, the neutral emission data take the form observed in Figure 4.57, with very evident time variations and range of magnitudes. There are two ways to try to condense this information in a way that may elucidate the physical processes. On one 
hand, these data have been used extensively for estimates of the diffusion properties of ions and neutrals in the substrate. This will be presented in sufficient detail in the following chapter. The other attempt has been the application of a simple desorption model which may be appropriate to describe what occurs at the surface of the emitter at low values of surface coverage. This is described in what follows. For low surface coverages, the neutral particle efflux per unit area $\left(v_{a}\right)$ may be modeled as:

$$
v_{a}(\theta, T)=\frac{\theta \sigma}{\tau_{0}} \exp \left(-\frac{Q}{k T}\right)
$$

where $\mathrm{Q}$ is the evaporation energy of the particle, $\theta$ is the fraction of a monolayer present in the surface, $\mathrm{T}$ is the temperature, $\sigma_{1}$ is the number of $\mathrm{Cs}$ atoms in a monolayer (= $\left.4.8^{*} 10^{14}\right)$, and $\tau_{0}$ is a constant parameter related to the mean lifetime of a neutral particle on the surface. Since our experimental conditions have a highly dynamical process in which $\theta$ is changing with time, the conditions are not appropriate for an accurate estimation of the parameter Q. Nevertheless, assuming a low $\theta$ where the variations of $Q$ are small, it is possible to make a crude estimate. Figure 4.59 shows the relevant data using average values obtained at different T's. From the slope of the curve, Q for Cs and $\mathrm{K}$ may be estimated as 1.39 and $0.93 \mathrm{eV}$ respectively. The value for $\mathrm{Cs}$ is close to but greater than the known value for a clean surface of $1.2 \mathrm{eV}$ (Langmuir, 1933). This is consistent with an expected increase of $\mathrm{Q}$ in the oxygenated tungsten surface, thus decreasing $v_{\mathrm{a}}$. The author is not aware of published values of $\mathrm{Q}$ for neutral $\mathrm{K}$. 


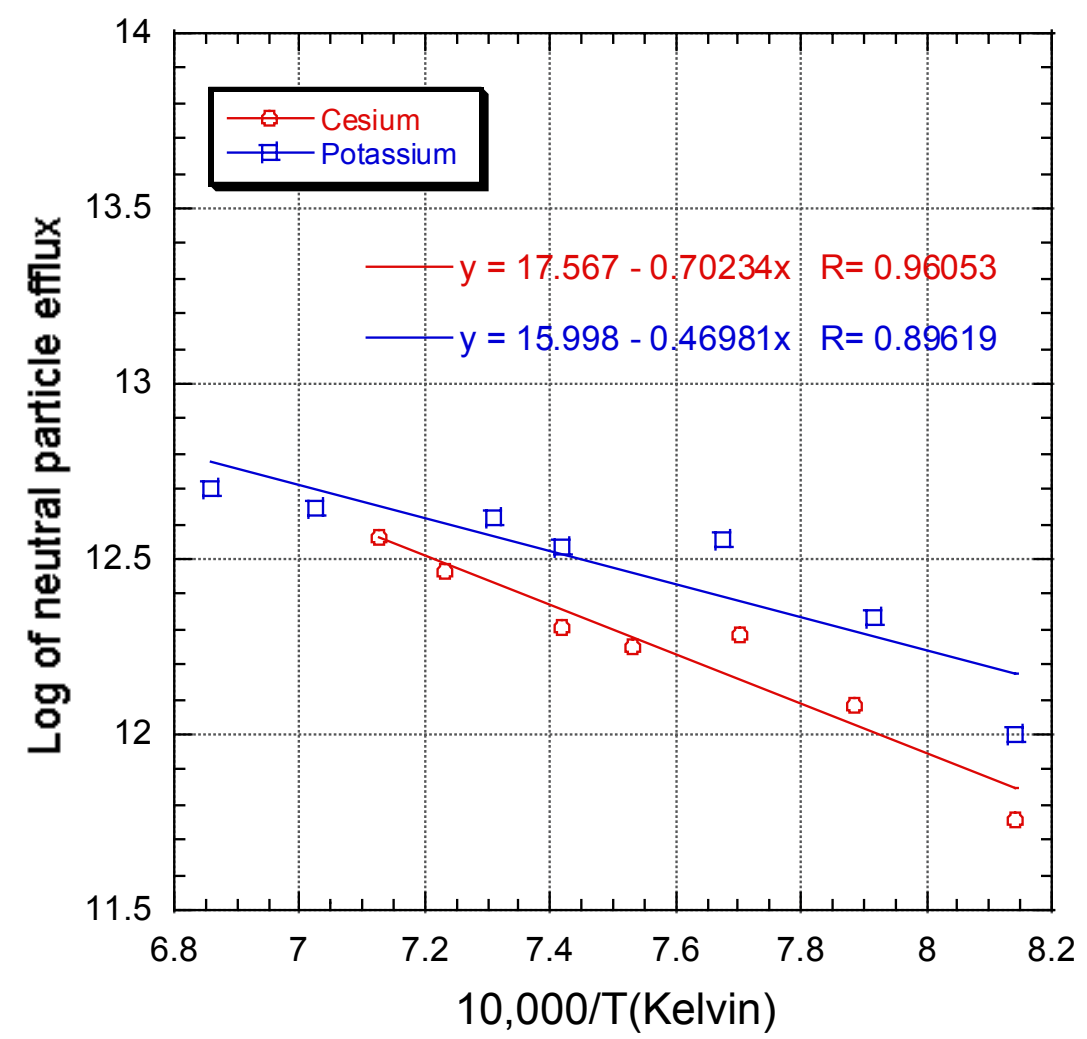

Figure 4.59. Data for a rough estimation of the desorption energies of Cs and K neutral particles.

These roughly calculated values of $\mathrm{Q}$ allow for an estimation of the relative change of neutral emission given a change in surface temperature. In general, these estimates were lower than what was actually observed by about $30 \%$. This could be qualitatively explained by an increase in $\theta$ due to an increased mobility of the atoms towards the surface.

The type of analysis made for the neutrals, based on an emission described by equation (4.6), assumes equilibrium conditions that are met to some extent given the slow changes in $\theta$. However, the pulsed conditions and high ion currents extracted during ion emission cause fast changes in $\theta$ during the pulse duration. A direct calculation of the ratio of the measured (pulsed) maximum ion emission rate $\left(v_{\mathrm{p}}\right)$ to $v_{\mathrm{a}}(\mathrm{DC})$ yields a curve 
that rapidly increases with $\mathrm{T}$ whereas equilibrium conditions described by the Saha equation would yield a ratio that decreases with $\mathrm{T}$ as shown in Figure 4.60.

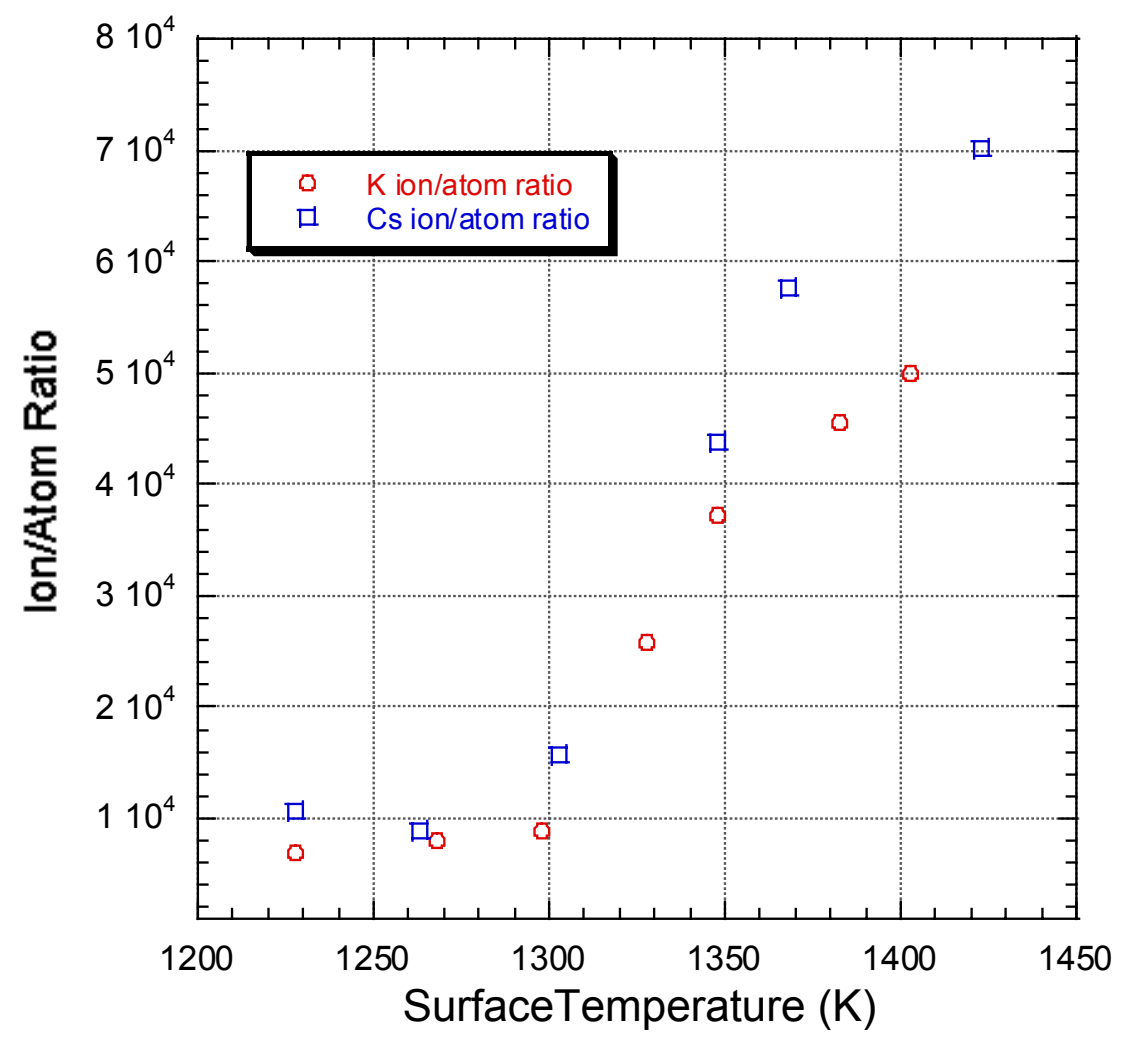

Figure 4.60. Ratio of the measured (pulsed) ion yield at different temperatures to the corresponding (DC) neutral particle emission rate.

This points to the fact that there is a diffusion limited flow of particles towards the surface of the emitter, which makes the neutral particle desorption low. However, during ion extraction the amount of particles present may be desorbed fast during the pulse, in a way that allows for the high current yields observed. Section 5.4.1 may be useful to understand the underlying mechanisms relevant in this case. 


\subsection{Integrated profile and emittance measurements of potassium doped and aluminosilicate sources.}

Among the diagnostics employed to characterize the ion beams described in these pages, one of the most informative is a double-slit setup that allows time-resolved measurements of the current profile as a function of the position perpendicular to the direction of propagation of the beam. This is done by moving a slit with a compact faraday cup behind it across the beam. This allows for assessing the beam intensity within the small area covered by the slit. The slit effectively integrates along one of the transverse directions, so, since the beam has a circular profile, the measured profiles have the shape of an inverted parabola even in the ideal case that the beam has uniform intensity. Commonly this is not the case. Figure 4.61 shows a profile measured at a position approximately $6.5 \mathrm{~cm}$ after the exit of the extraction plate aperture. The measurement was made during a fast heat-up cycle of a potassium doped source.

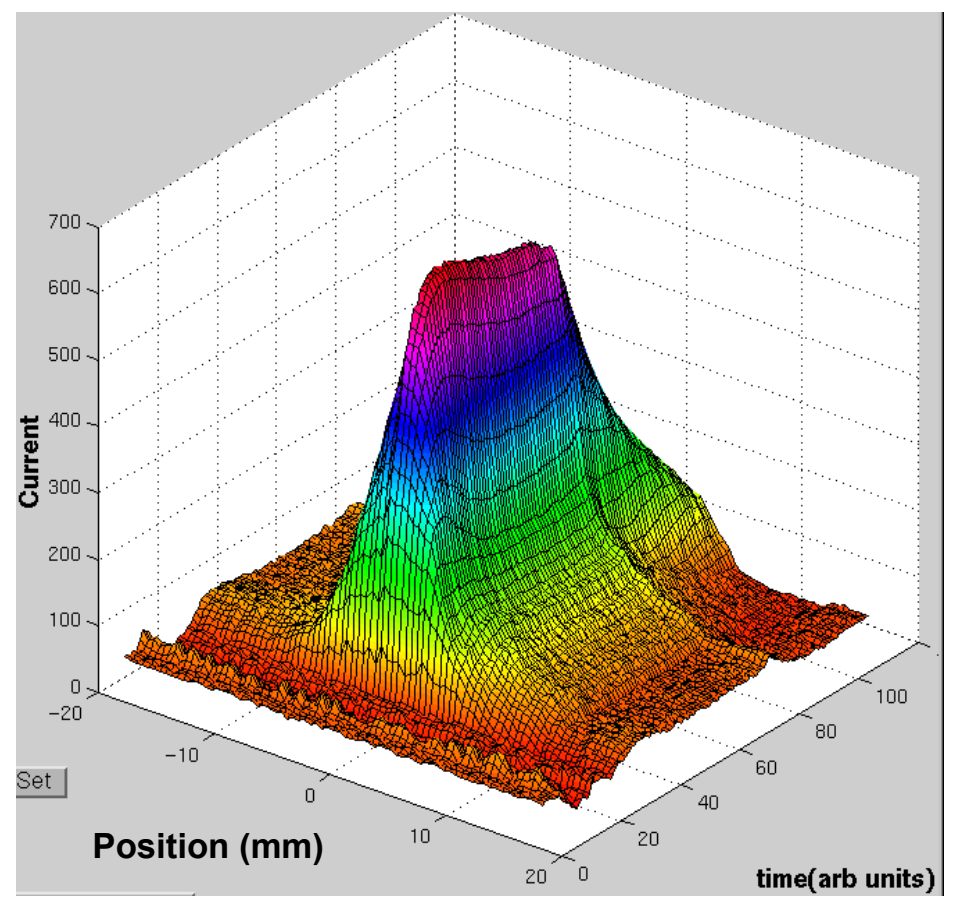

Figure 4.61. Slit-cup signal as a function of position and time of a potassium doped source operating at $1,045{ }^{\circ} \mathrm{C} . \mathrm{I}=1.24 \mathrm{~mA} . \quad<\mathrm{J}>=4.4 \mathrm{~mA} / \mathrm{cm}^{2}$. 
The vertical axis on the previous figure is the measured signal in $\mathrm{mV}$ at the small faraday cup behind the slit. These measurements were carried out in the "reverse bias" mode, meaning that a negative potential $(-400 \mathrm{~V})$ was applied to the small faraday cup collector, while a positive $(+400 \mathrm{~V})$ was applied to the grid in front of it. The result is that the measured signal is amplified by the secondary electrons released when the energetic ions impinge on the collector. The adjustment into a current value from the voltage signals measured requires two factors: division by the gain of the used amplifier, in this case 226, and adjustment with the secondary electron coefficient of the used collector material and at the respective ion energy. In these tests, the generic voltage signal is plotted. The total ion beam current is measured separately by a large faraday cup that catches the whole beam.

The time axis in these plots appears usually as the numbering of the points acquired from the scope trace, which has not been fully sampled to reduced the size of the data files. All the short pulses used in the tests described on these pages have the same pulse length, about $2.3 \mu \mathrm{s}$.

Figure 4.62 shows a time slice at the middle of the pulse described in the previous figure. As mentioned above, these tests were done during a fast heating cycle, which means that the source was at operating temperature before it actually had attained the sufficiently low surface coverage for maximum ion yield. The asymmetry observed is due to the increased ion current during the time of the measurement. Such effect is not observed when more stable conditions of operation are attained. Figure 4.63 shows a 
similar time resolved profile of the same source operating at a higher temperature of $1,075 \mathrm{C}$, under better potassium surface coverage conditions.

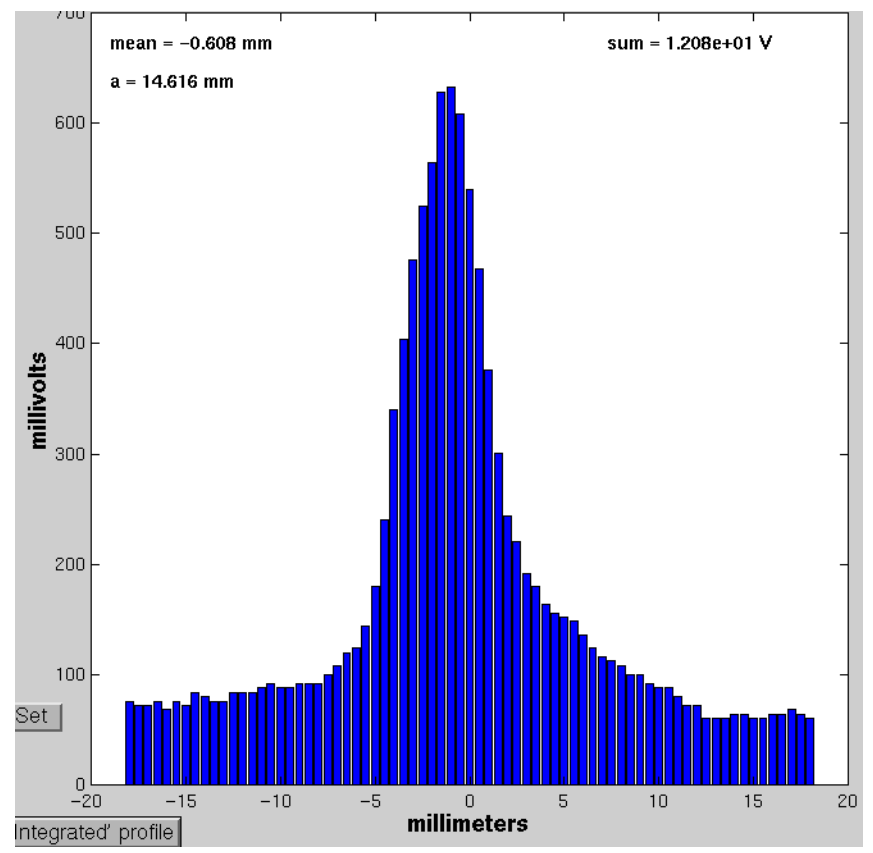

Figure 4.62. Beam profile at mid pulse of the potassium beam. The asymmetry of the beam is partially due to an increase of the beam current with time. $\mathrm{T}=1,045^{\circ} \mathrm{C}$.

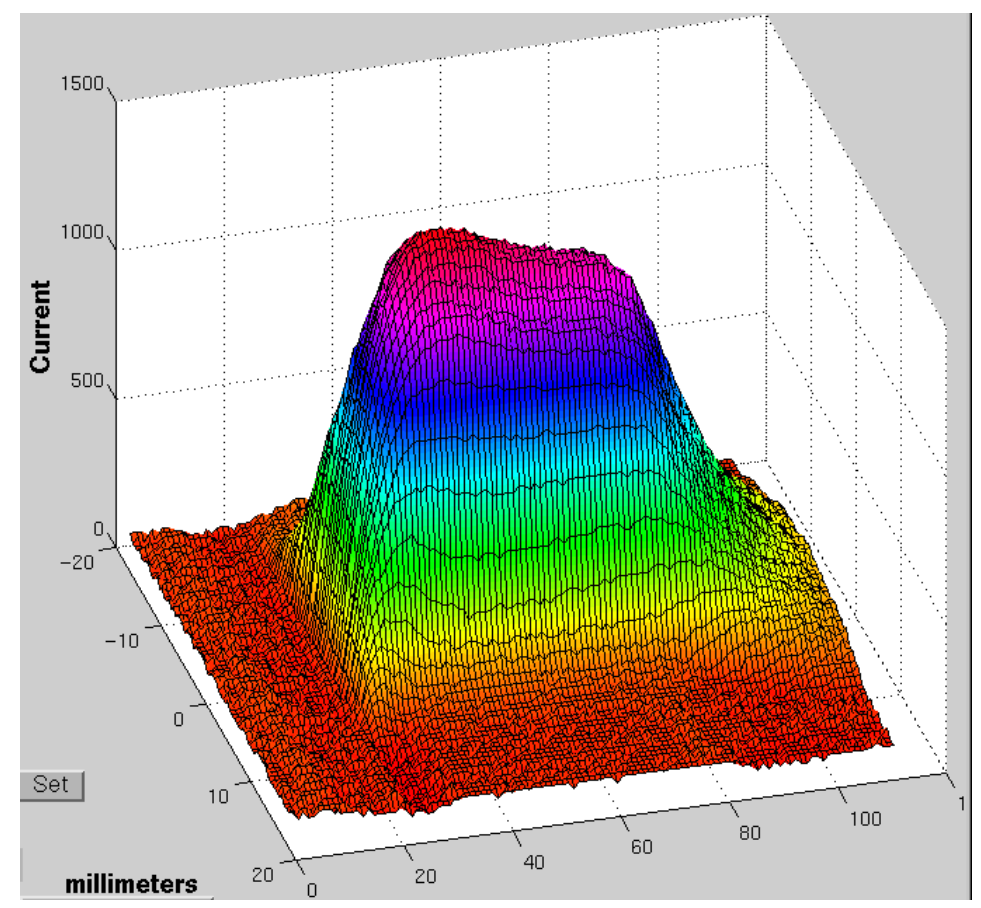

Figure 4.63. Time-resolved beam profile of the potassium beam. $\mathrm{T}=1,075^{\circ} \mathrm{C} . \mathrm{I}=4$ $\mathrm{mA} .<\mathrm{J}>=14.18 \mathrm{~mA} / \mathrm{cm}^{2}$. 


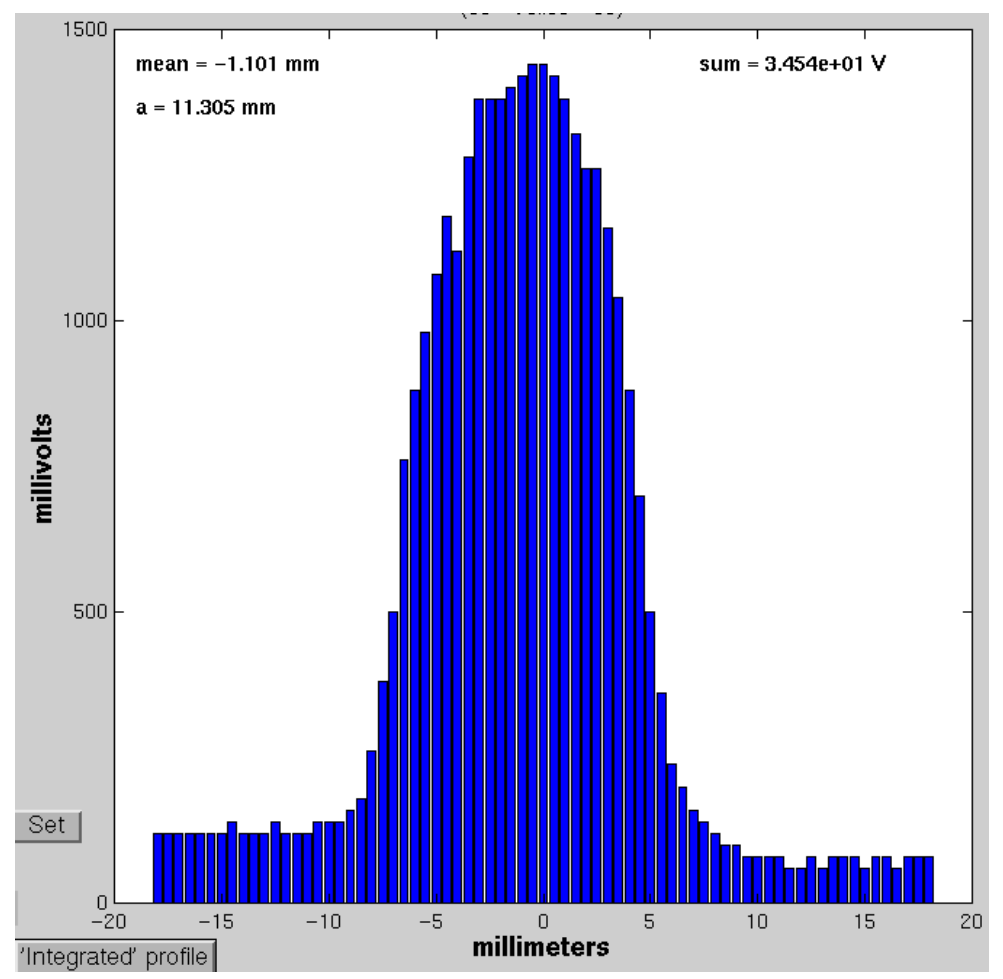

Figure 4.64. Beam profile at mid pulse of the potassium beam. $\mathrm{T}=1,075{ }^{\circ} \mathrm{C}$.

The double slit diagnostic allows for measuring the beam's phase space in a direction transverse to the direction of propagation. A sample of one such measurement for a potassium-doped source operating at the conditions described by the Figures 4.63 and 4.64 is shown in Figure 4.65. In addition to the value of the 4rms $(\varepsilon)$ emittance noted in the plot, the normalized emittance $\left(\varepsilon_{\mathrm{n}}=\beta \gamma \varepsilon\right)$, is also presented. The emittance measurement is also time-resolved. The plots correspond to the emittance measurement during the flatter section of the pulse, and consider only that data that eliminates the constant offset generated by the amplifier used. The enclosed beam fraction will correspond then to values between $90 \%$ and $95 \%$ of the total. Figure 4.66 shows the same plot as in the previous figure, but this time adjusts the vertical values with the transformation $\mathrm{x}_{\text {sheared }}=\mathrm{x}^{\prime}{ }_{\text {original }}-\mathrm{x}<\mathrm{x} \cdot \mathrm{x}^{\prime}{ }_{\text {original }}>/<\mathrm{x}^{2}>$ where the brackets indicate RMS values. 


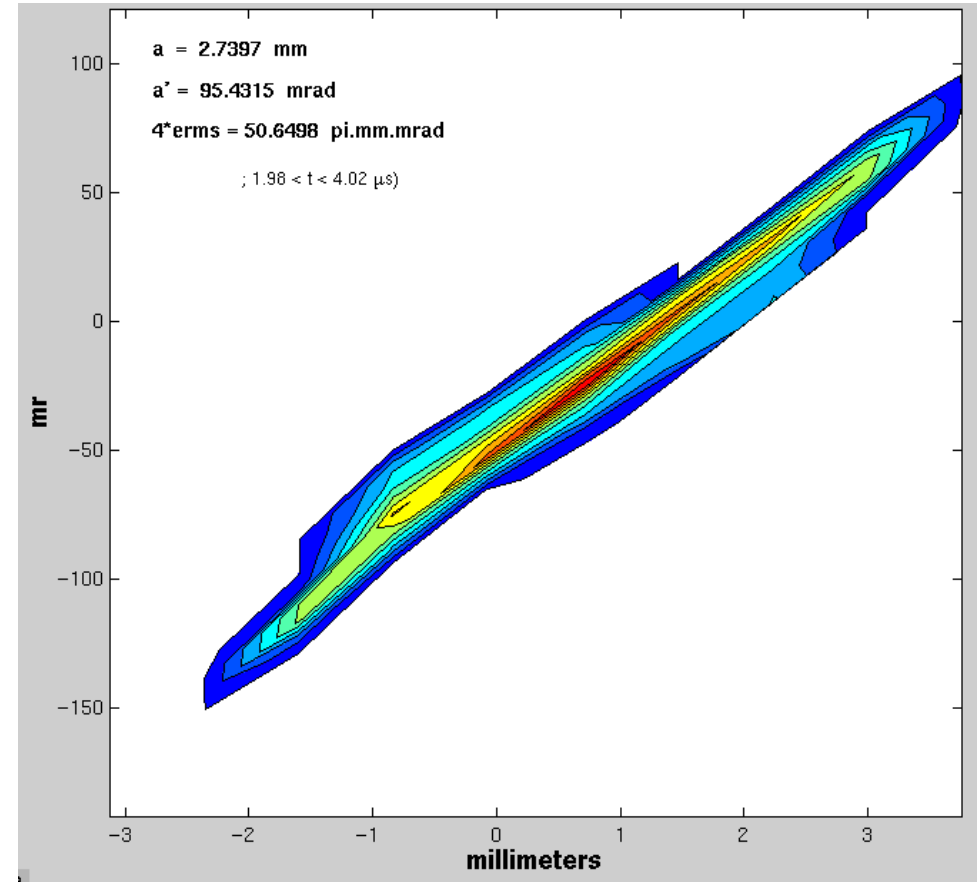

Figure 4.65 Phase space plot of the potassium beam. Source temperature: $1,075^{\circ} \mathrm{C}$. Normalized emittance: $6.14 \cdot 10^{-2} \pi \mathrm{mm}$ mrad.

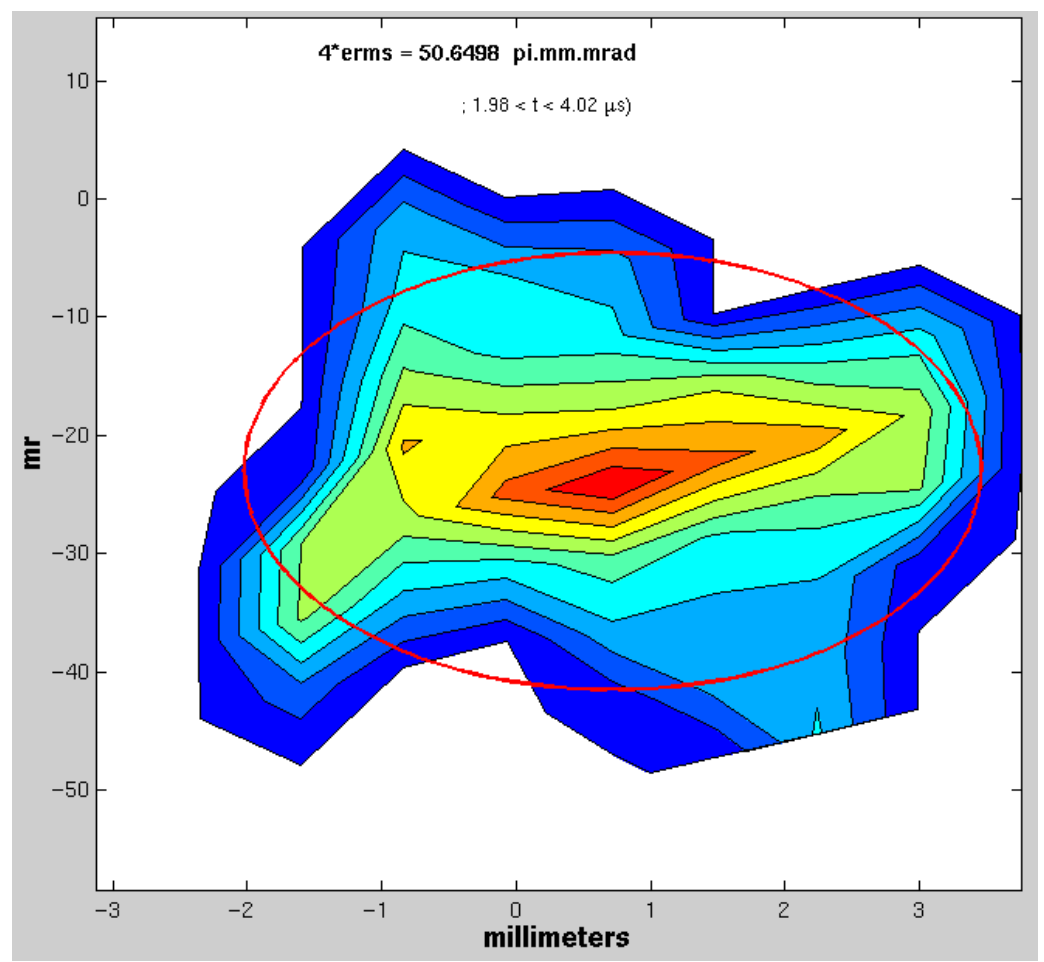

Figure 4.66. Same phase space as in Figure 4.64 but sheared to appreciate structure. 
Upon improvement of the surface coverage of potassium atoms with time, the current yield increases with time even though the temperature is maintained. Figure 4.67 shows another time-resolved profile operating at a temperature of $1,075{ }^{\circ} \mathrm{C}$. Contrast with Figure 4.63 where the current had roughly half the magnitude. The increase in beam diameter may also be seen when comparing figures 4.64 and 4.68 showing the profile at mid-pulse.

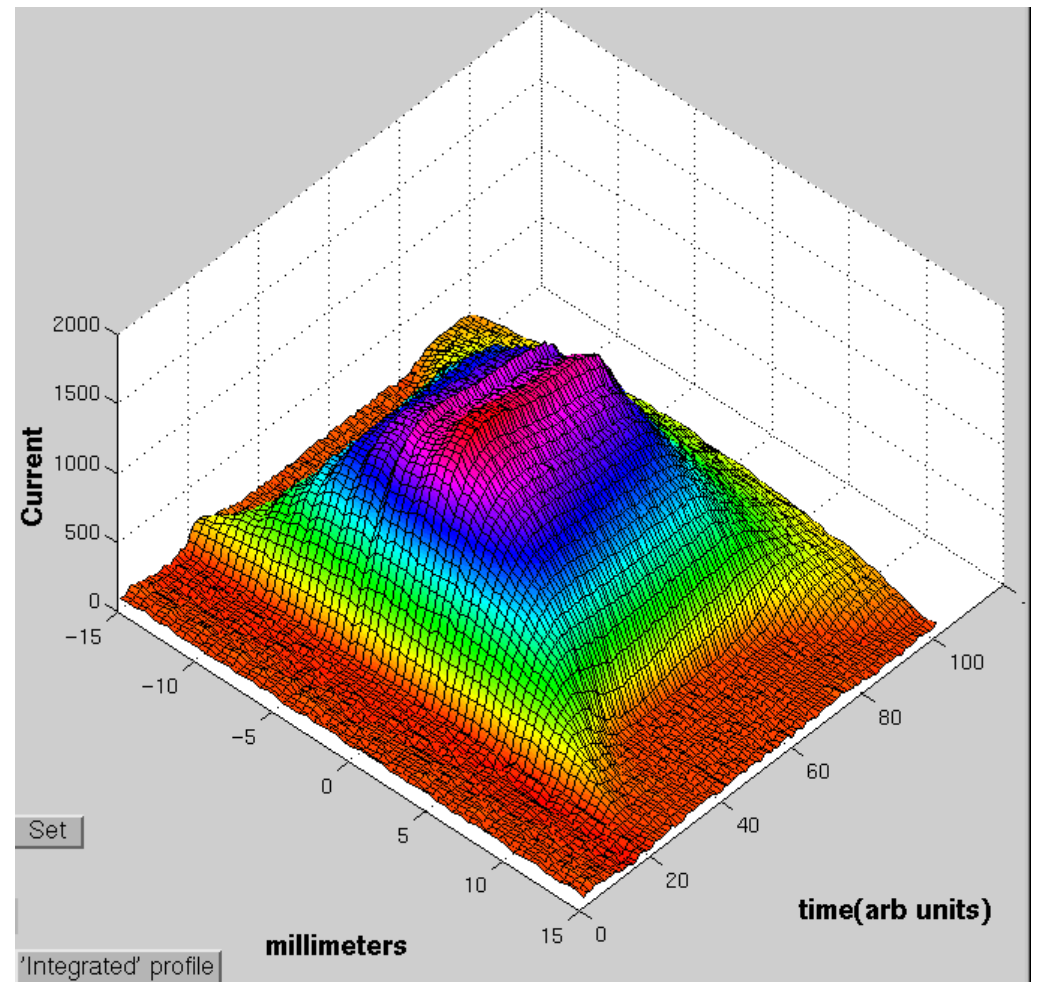

Figure 4.67. Time-resolved beam profile of the potassium beam. $\mathrm{T}=1,075^{\circ} \mathrm{C} . \quad \mathrm{I}=8.6$ $\mathrm{mA} .<\mathrm{J}>=30.5 \mathrm{~mA} / \mathrm{cm}^{2}$. Current is higher due to better surface coverage.

Figure 4.69 shows yet another time-resolved current profile. The increase in current has been caused by increasing the operating temperature, in this case to $1,150{ }^{\circ} \mathrm{C}$. The following figure shows a slight asymmetry in the profile of this beam, most likely caused by a misalignment of the emitter within the Pierce structure. 


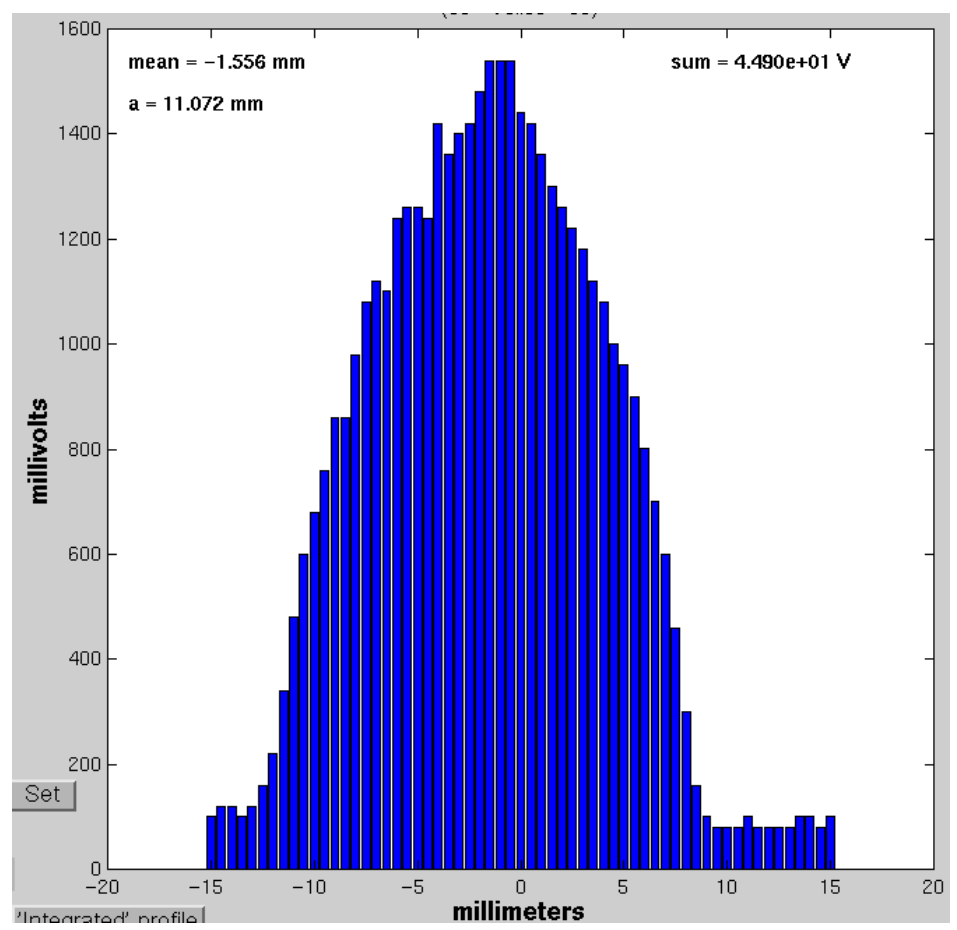

Figure 4.68. Beam profile at mid pulse of the potassium beam after improvement of the surface coverage conditions. $\mathrm{T}=1,075^{\circ} \mathrm{C}$.

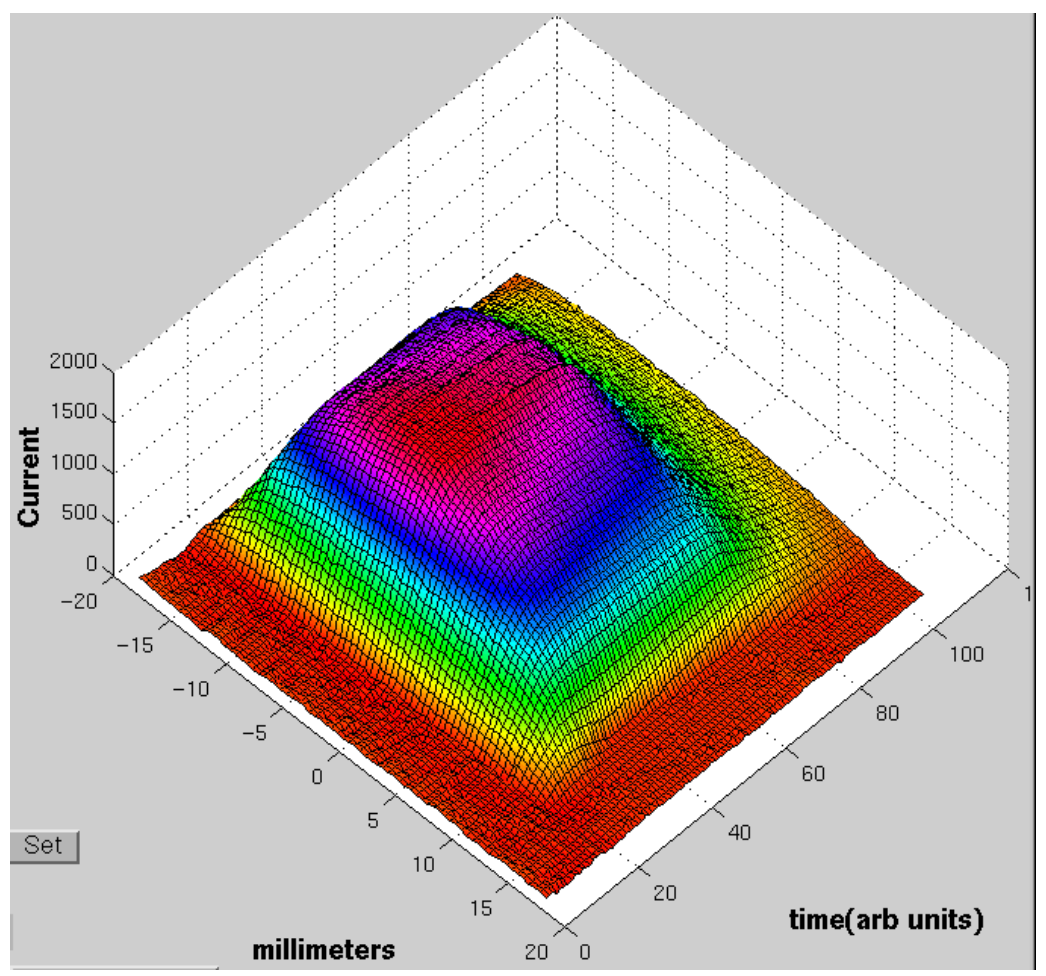

Figure 4.69. Time-resolved beam profile of the potassium beam. $\mathrm{T}=1,150{ }^{\circ} \mathrm{C} . \mathrm{I}=11.9$ $\mathrm{mA} .<\mathrm{J}>=42.2 \mathrm{~mA} / \mathrm{cm}^{2}$. 


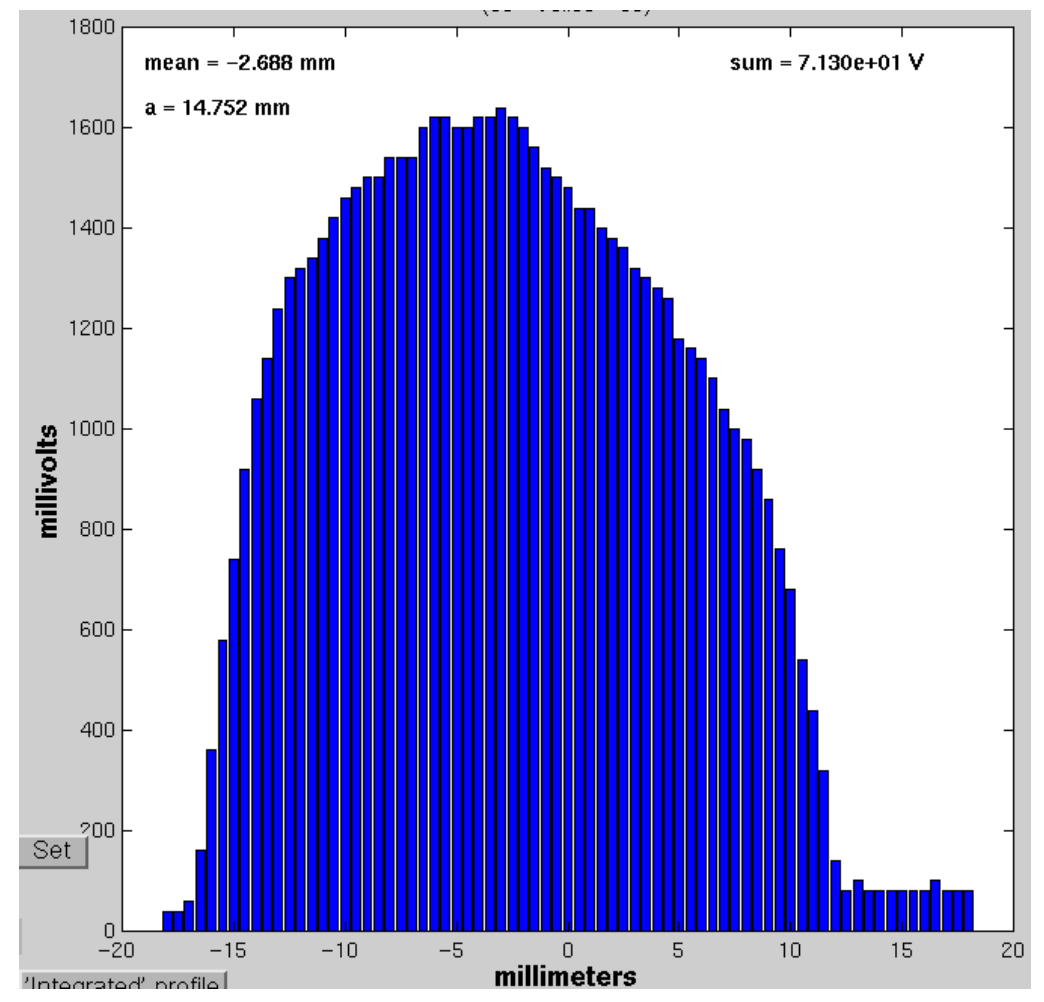

Figure 4.70. Beam profile at mid pulse of the potassium beam. $\mathrm{T}=1,150{ }^{\circ} \mathrm{C}$.

Figure 4.71 shows the emittance plot corresponding to the beam shown in figures 4.69 and 4.70. The irregularities observed in the last phase space plot have disappeared, yielding a beam with a significantly lower emittance. As in the previous case, only the flatter part of the beam pulse has been considered, and the lowest level signal corresponding to the DC offset produced by the electronic amplifier has been ignored. Figures 4.72 and 4.73 show the profile and emittance plot of the potassium beam operating at $1,205^{\circ} \mathrm{C}$ and at a higher extraction voltage. Given the values of current and emittance, it can be seen that these are very bright beams. The magnitude of the brightness is comparable to the desired values for a multi-beamlet architecture, depending on how high an initial emittance is allowed in these designs. See the end of this section for Table 4.1, a summary of the results presented in these pages. 


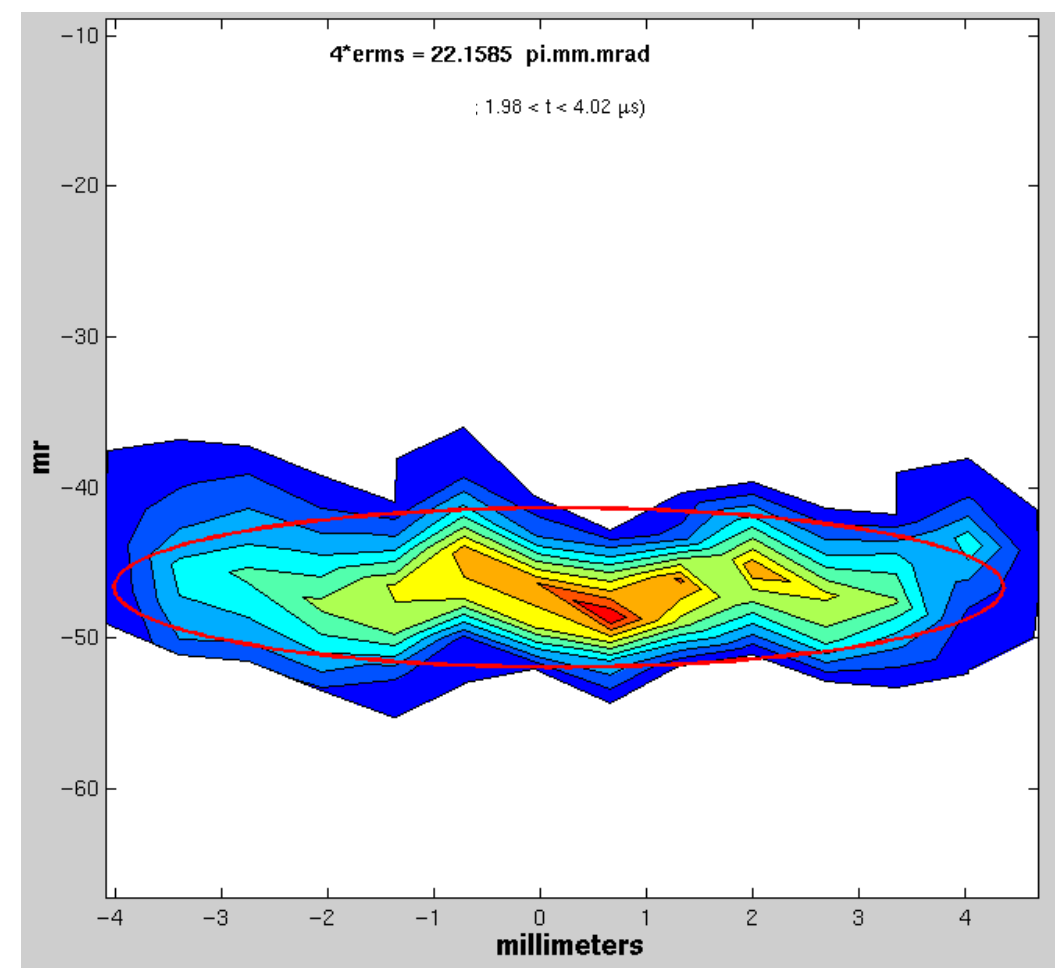

Figure 4.71 Phase space plot of the potassium beam. Source temperature: $1,150{ }^{\circ} \mathrm{C}$. Normalized emittance: $2.69 \cdot 10^{-2} \pi \mathrm{mm}$ mrad.

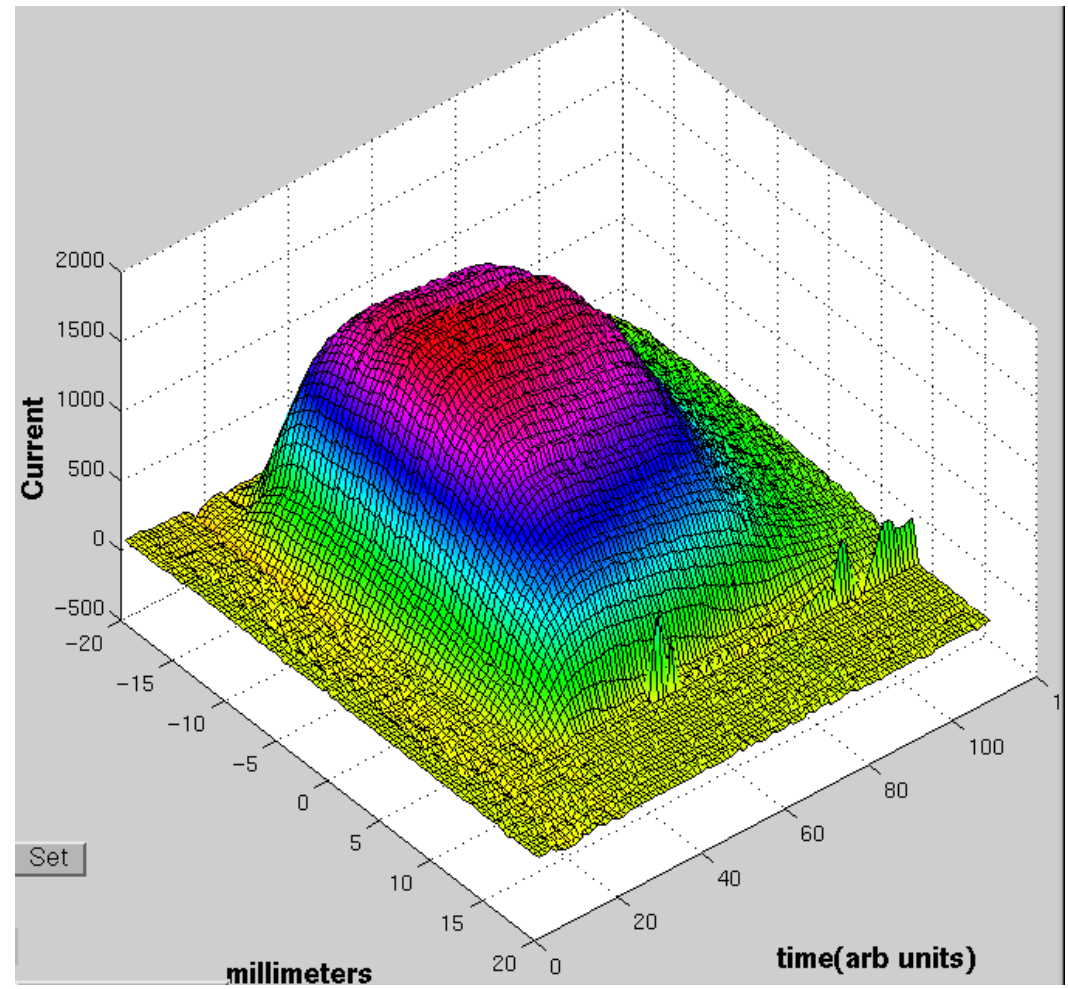

Figure 4.72. Time-resolved beam profile of the potassium beam. $\mathrm{T}=1,205^{\circ} \mathrm{C} . \mathrm{I}=$ $12.44 \mathrm{~mA} .<\mathrm{J}>=44.1 \mathrm{~mA} / \mathrm{cm}^{2}$. 


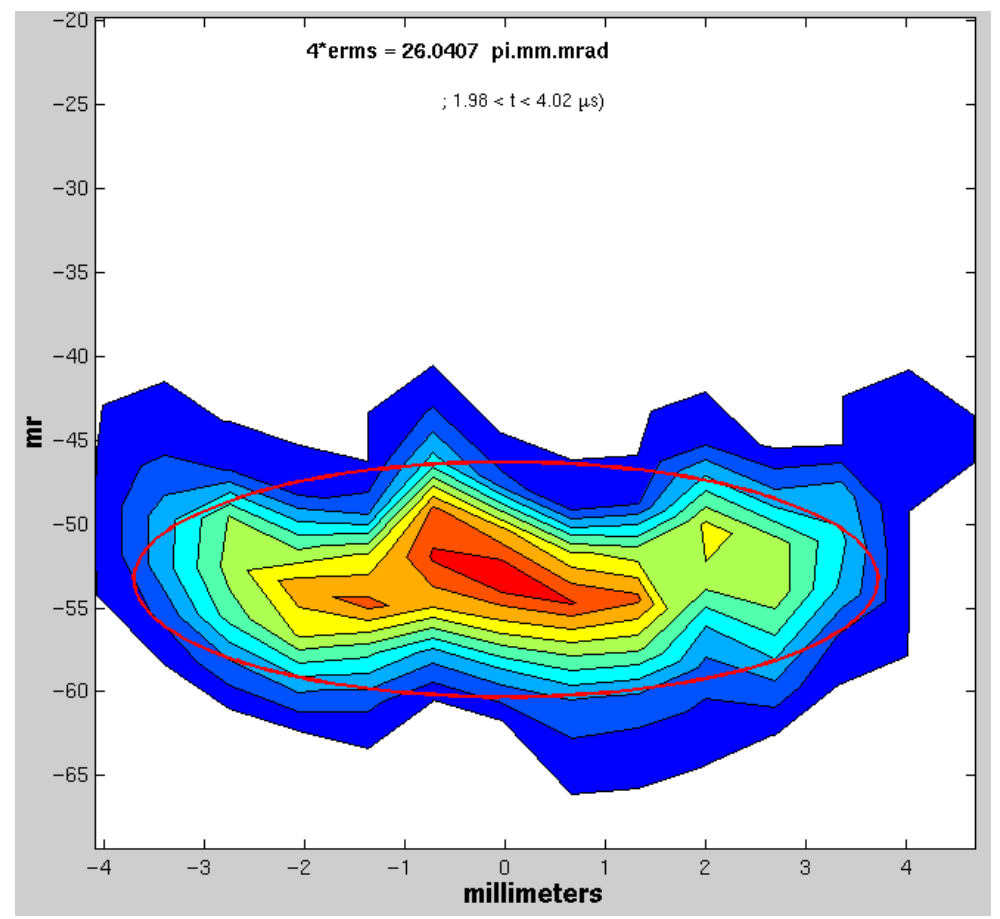

Figure 4.73 Phase space plot of the potassium beam. Source temperature: $1,205{ }^{\circ} \mathrm{C}$. Normalized emittance: $3.44 \cdot 10^{-2} \pi \mathrm{mm}$ mrad.

It may be of interest to see the characteristic signature of a depleting ion beam, occurring when the pulse starts to droop during the pulse time. Figure 4.74 shows this behavior occurring at all the positions within the beam. The total beam behavior, as measured by the large faraday cup is presented in Figure 4.75. 


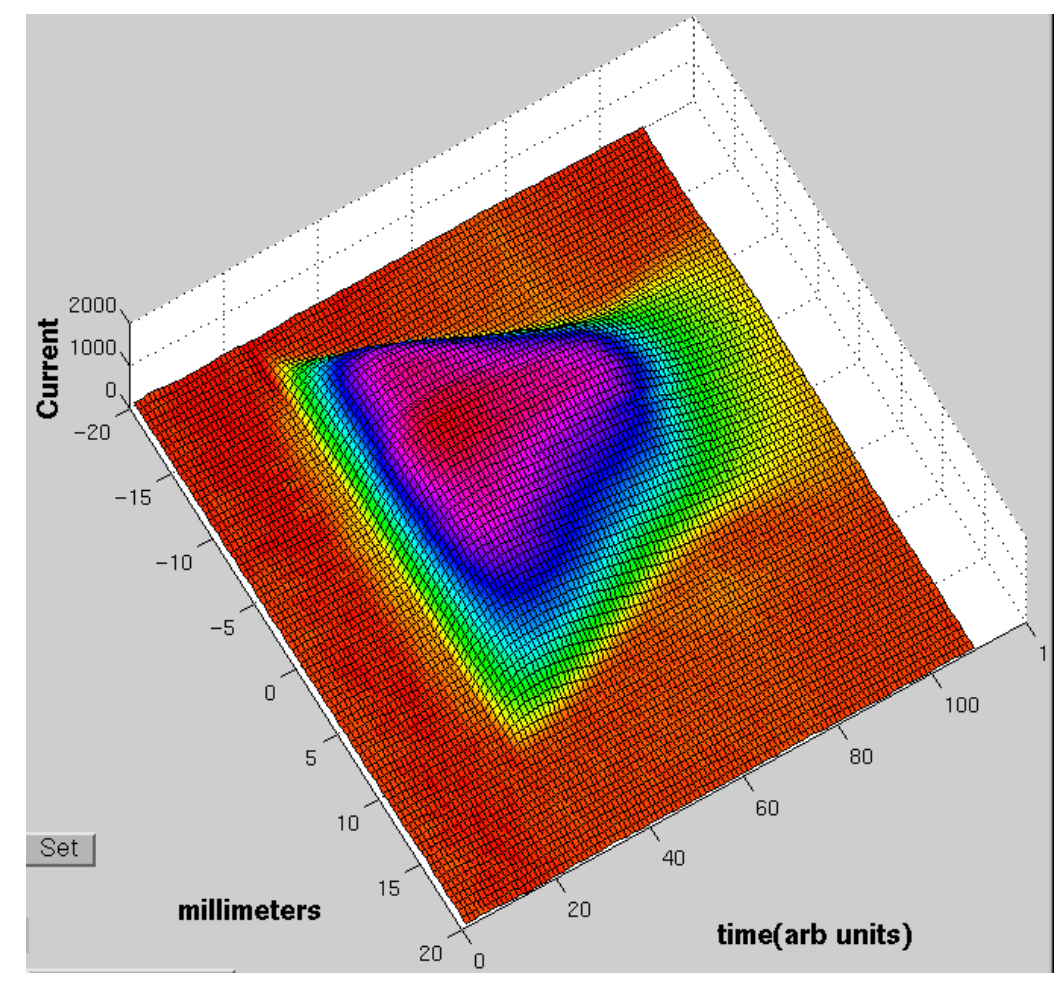

Figure 4.74. Time-resolved beam profile showing the characteristic depletion features.

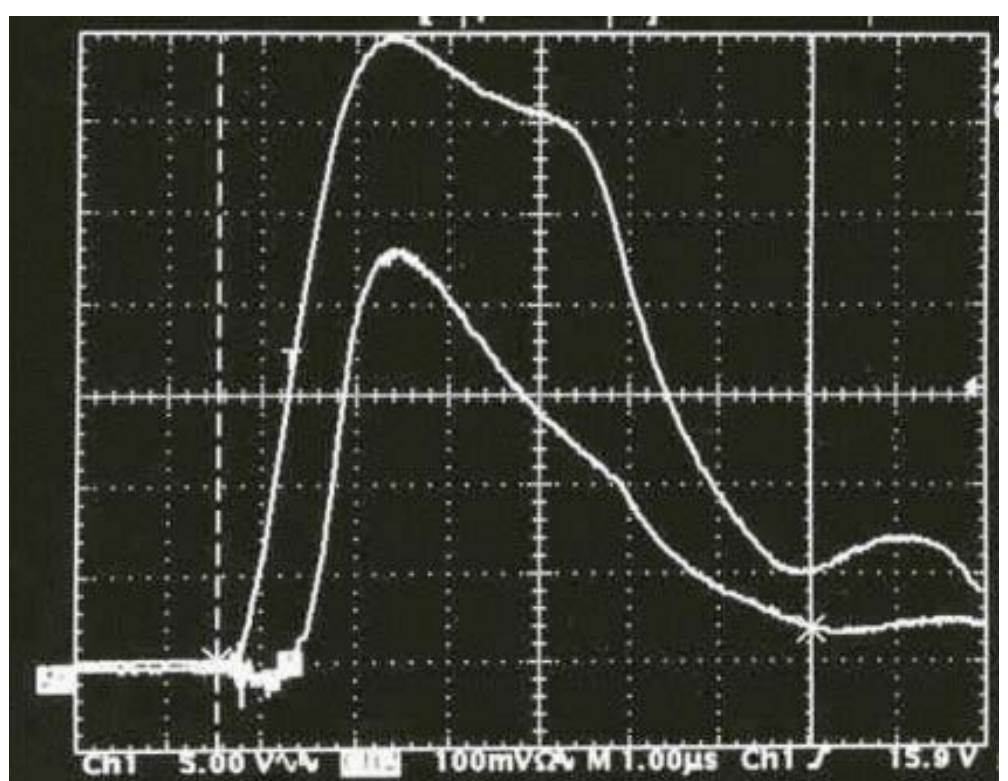

Figure 4.75. Oscilloscope traces showing the short $(\sim 2 \mu \mathrm{s})$ high voltage pulse (upper trace) and the faraday cup signal (lower trace) of a depleting ion source.

Similar analyses were carried out in the case of aluminosilicate ion sources. The results are presented in the following pages. Figure 4.76 shows a plot summarizing the 
results of all the measured profiles (at mid pulse) for this type of source, as the profiles vary with the operating temperature. The same changes in terms of beam intensity and beam diameter are observed as the current increases. In contrast with the previous example of a potassium doped emitter, the aluminosilicate source displays a characteristic irregular emission patterns that vary with temperature as different regions become more able to deliver a particular value of current.

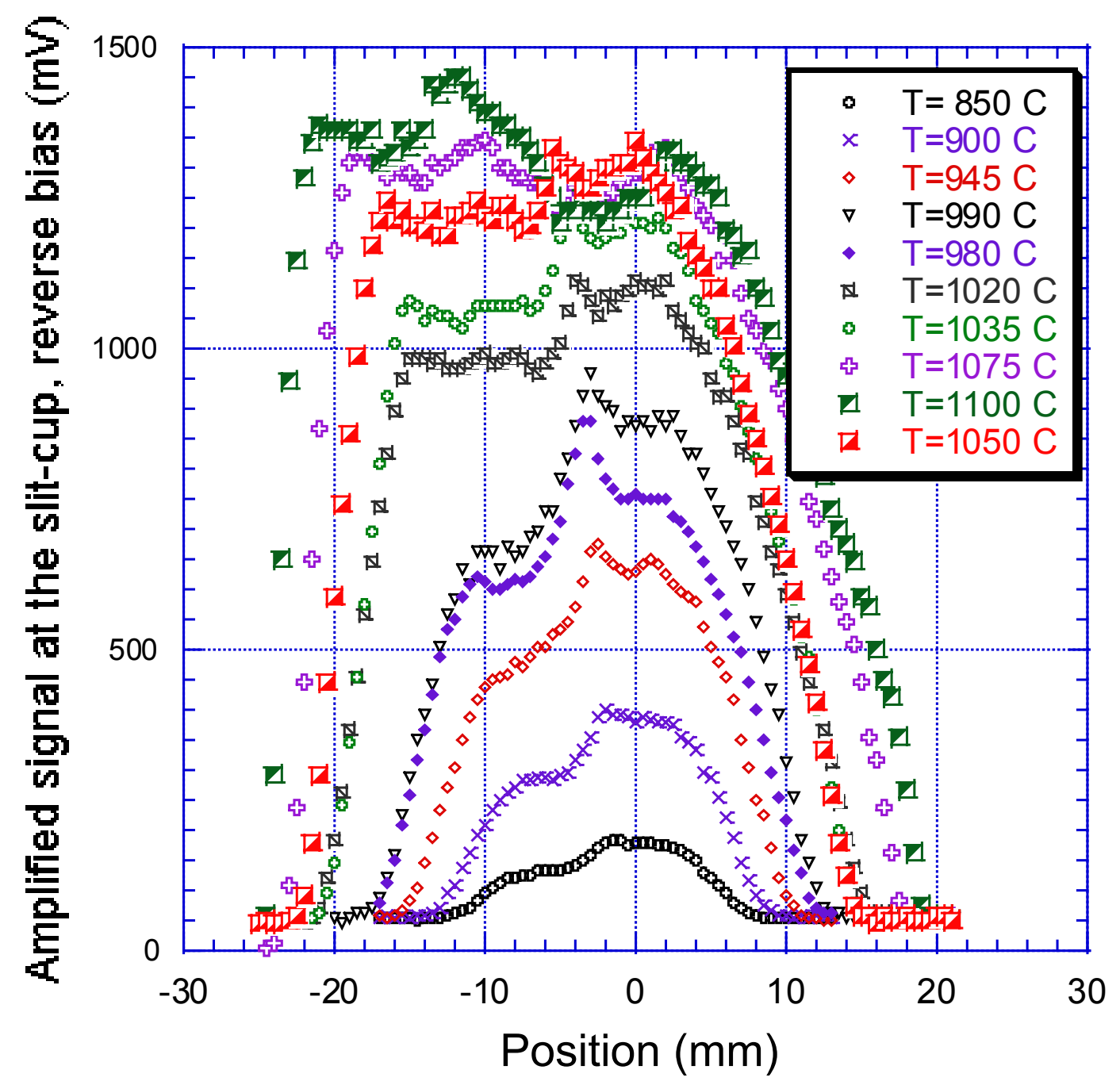

Figure 4.76. Family of mid-pulse profiles at a fixed voltage of a potassium aluminosilicate type source, at different temperatures of operation. 
Figure 4.77. Shows a summary of the total current output of the ion source as a function of operating temperature. In order to make a conversion to the corresponding average current density it is necessary to know that the emitter area is $0.282 \mathrm{~cm}^{2}$. Figure 4.78 shows the corresponding values of the normalized emittance. This plot shows the particular feature that the emittance, as a general trend in this plot, decreases with temperature. A reasonable explanation for this behavior has to do with the increased and presumably less spotty current emission as the temperature increases. This more uniform emission may minimize the transverse particle motion that is provoked when the nonuniformity in the emission is very large. The result of these graphs is that as the temperature rises and the current increases, the brightness will increase as well.

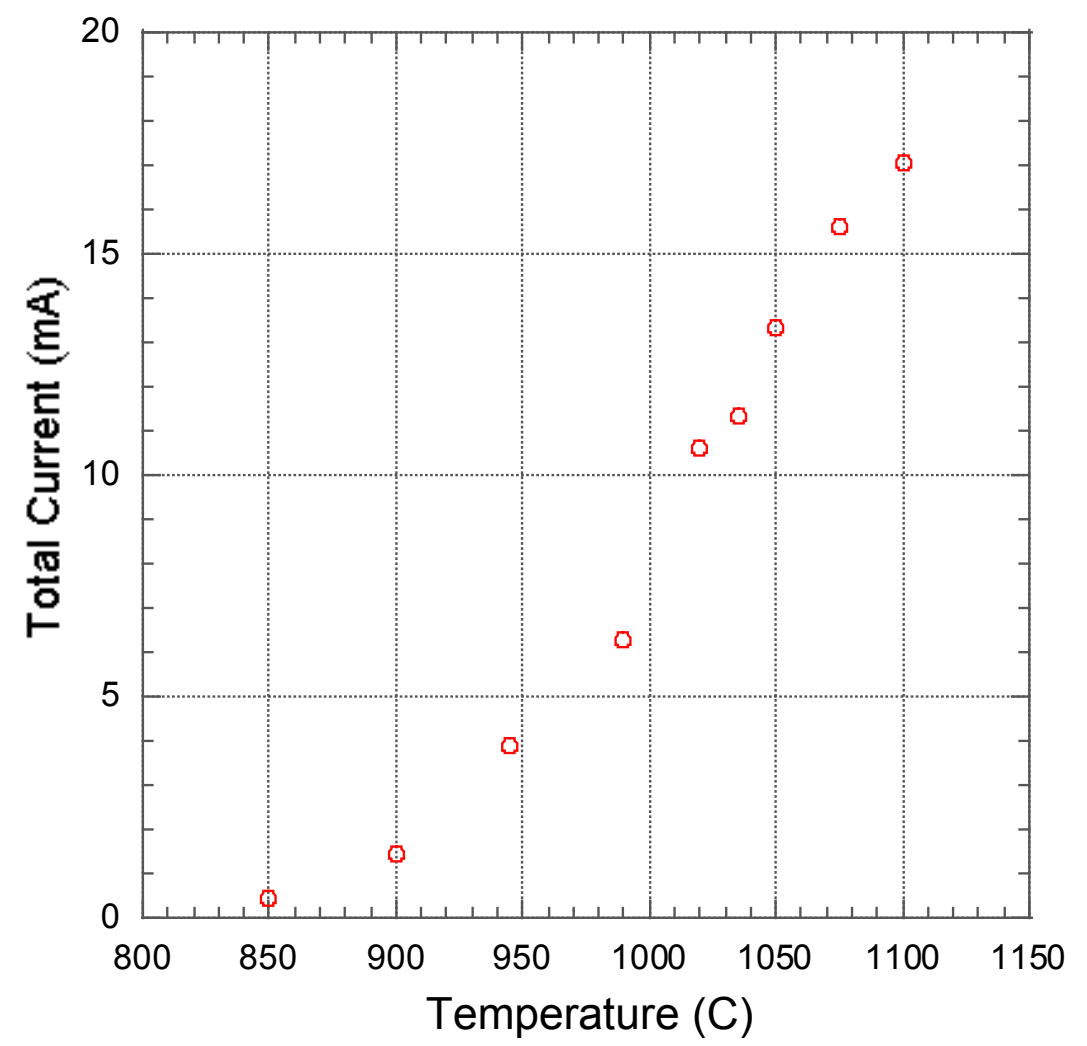

Figure 4.77 Current emission from a potassium aluminosilicate source as a function of surface temperature. 


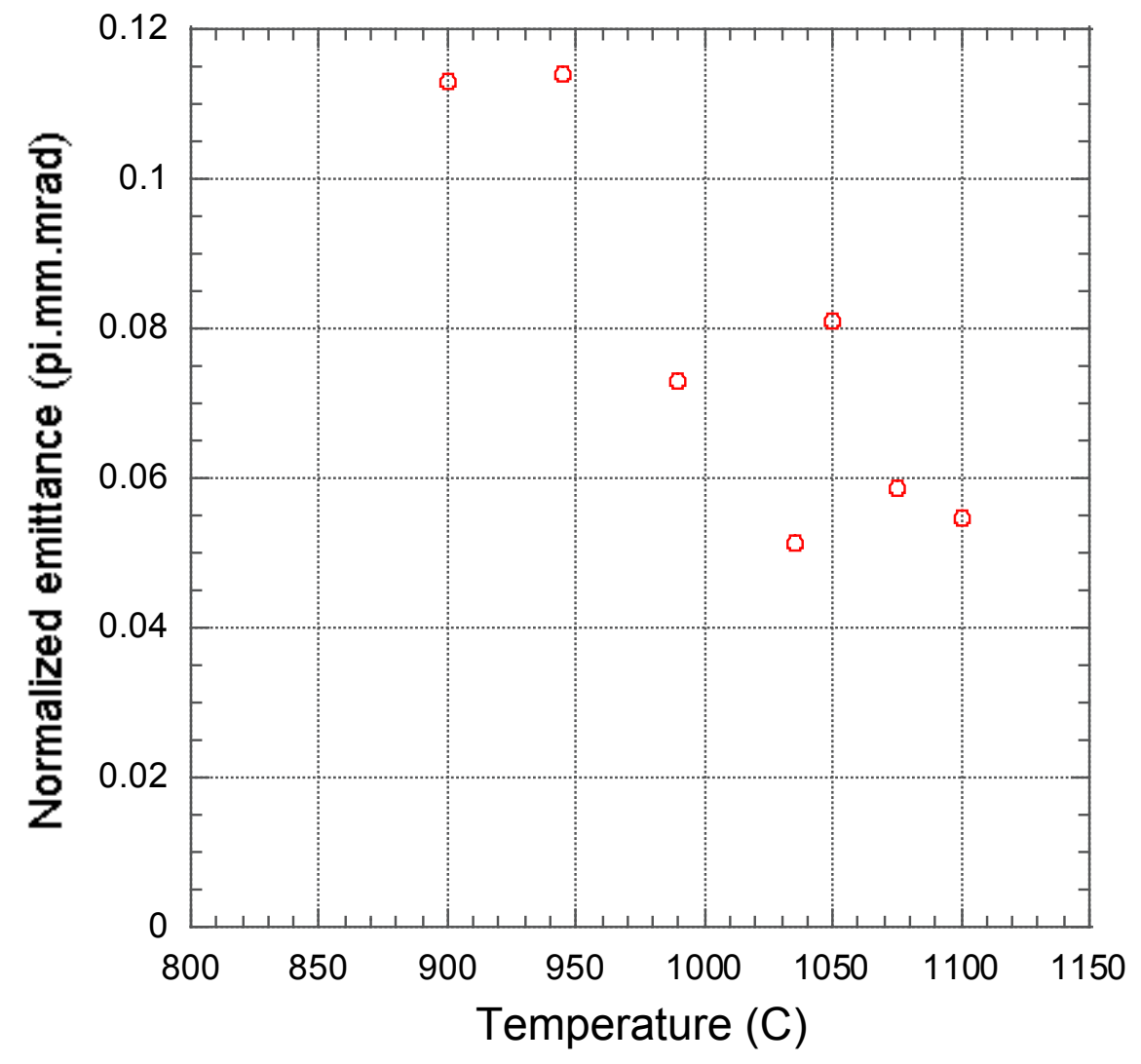

Figure 4.78 Current emission from a potassium aluminosilicate source as a function of surface temperature.

Figure 4.79 shows the measured emittance plot for the K-aluminosilicate source at $945^{\circ} \mathrm{C}$. From the irregularities on the plot it is quite clear why the emittance is so large. Figures 4.80 through 4.83 show additional examples of time-resolved profile measurements and emittance plots. The highly irregular character observed on Figure 4.79 is minimized and the emittance decreases as the temperature goes up. 


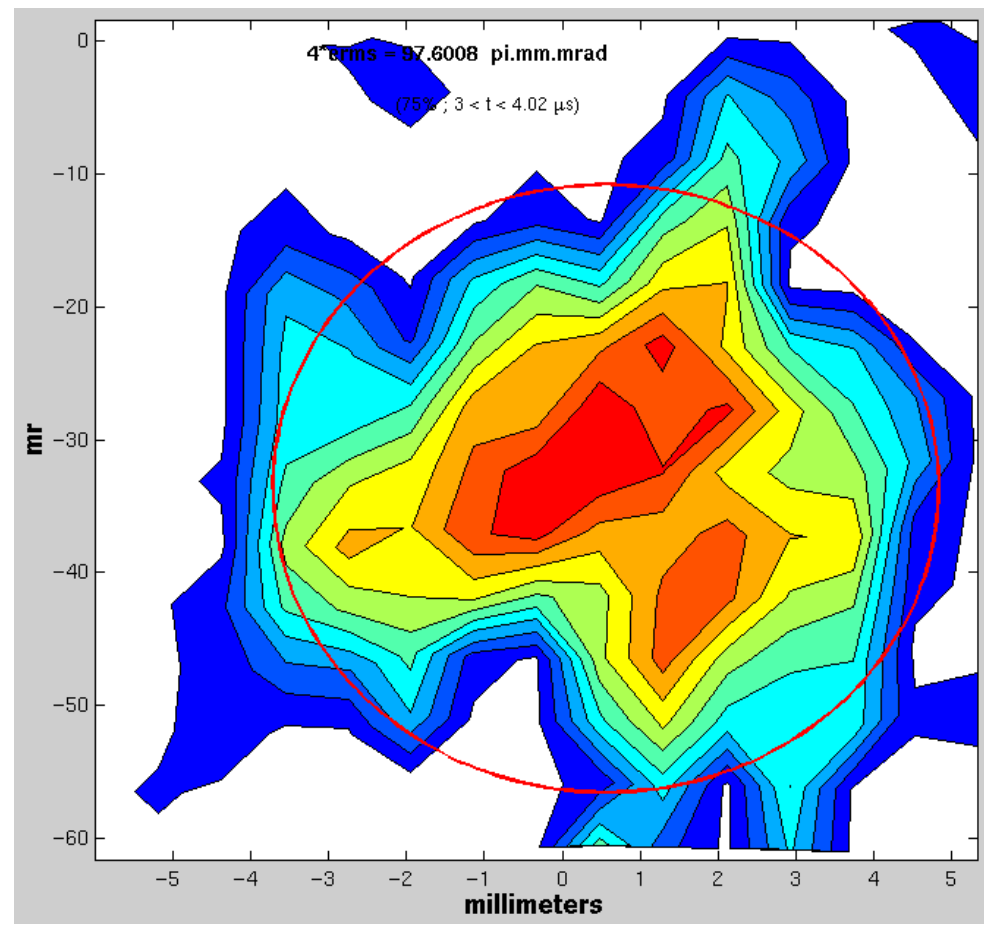

Figure 4.79. Emittance plot of a potassium aluminosilicate source operating at $945 \mathrm{C}$.

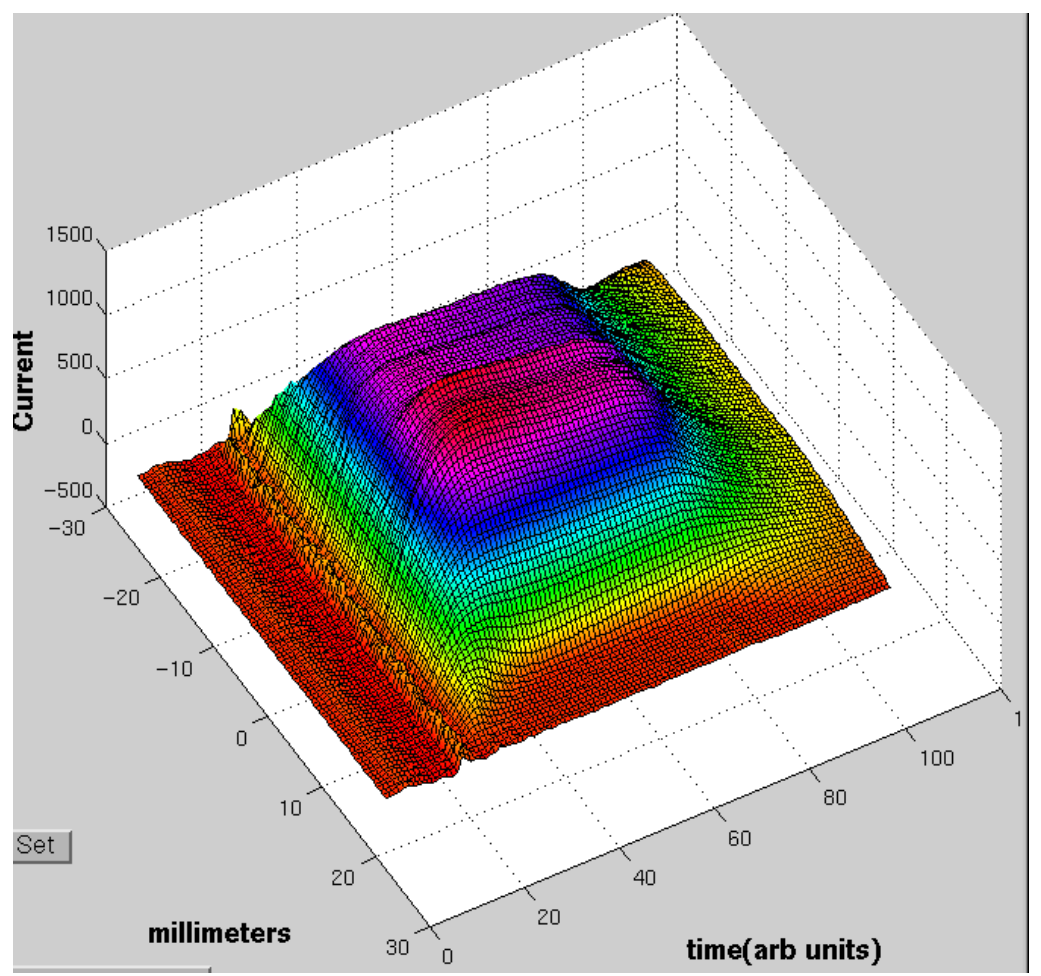

Figure 4.80 Time resolved profile of a potassium aluminosilicate source. $\mathrm{T}=1,035^{\circ} \mathrm{C}$. 


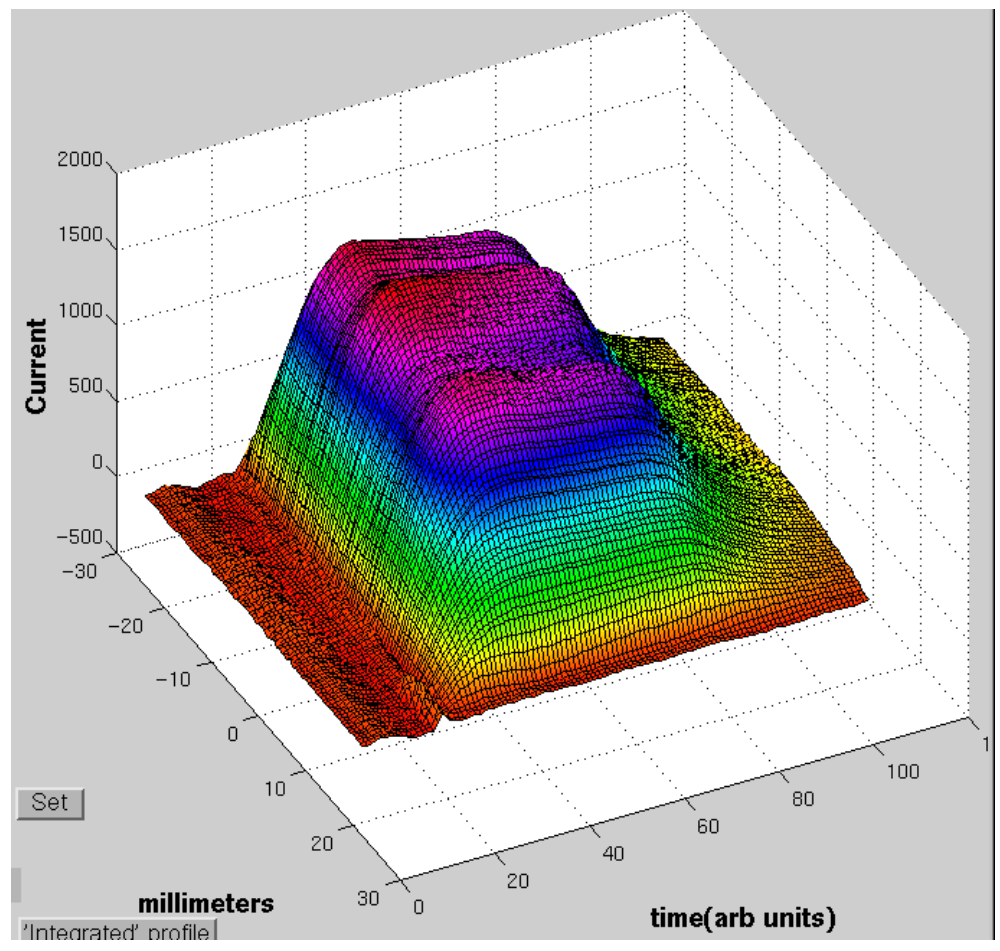

Figure 4.81 Time resolved profile of a potassium aluminosilicate source. $\mathrm{T}=1,100{ }^{\circ} \mathrm{C}$.

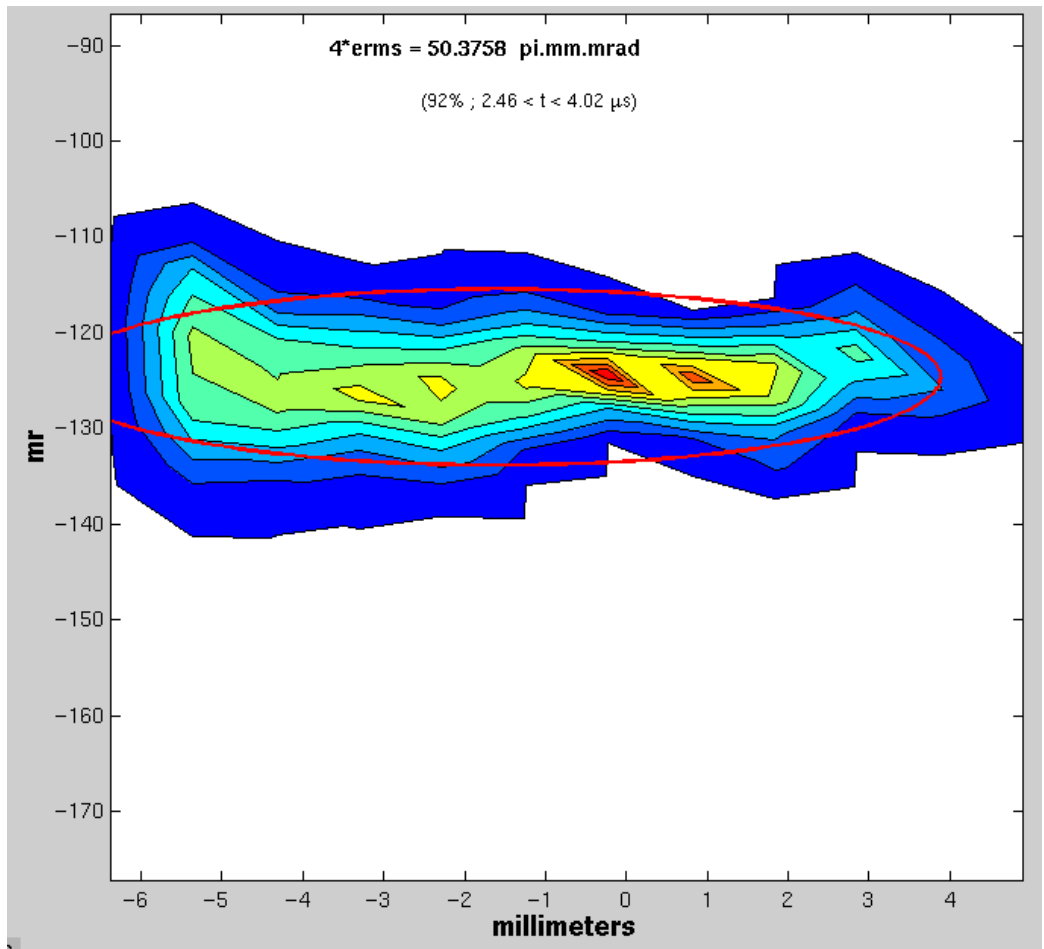

Figure 4.82 Phase space measurement of a potassium aluminosilicate source. $\mathrm{T}=1,075$ ${ }^{\circ} \mathrm{C}$. 


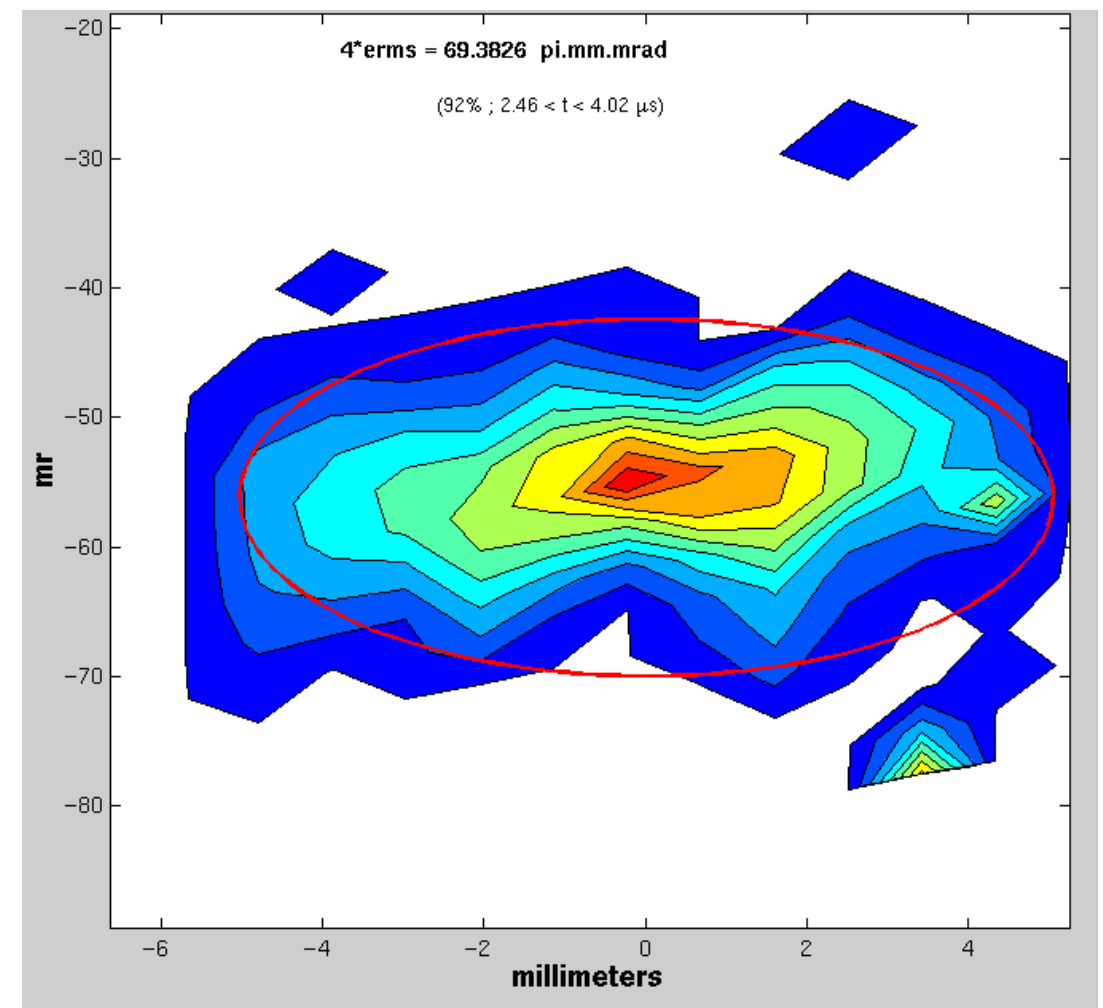

Figure 4.83 Phase space measurement of a potassium aluminosilicate source. $\mathrm{T}=1,050$ ${ }^{\circ} \mathrm{C}$.

Section 5.7 presents some computer simulations of the expected space chargelimited beam profiles. The graphs of the beam profiles presented in Section 5.7 are not integrated. In other words, those graphs represent the true expected dependence of $\mathrm{J}$ with respect to the radial position. For this reason, a perfectly uniform beam would have a flat top with a sharp drop at the edge. For this reason the graphs of the simulations and the measured profiles are not directly comparable. The measured profiles have been integrated in the direction perpendicular to the direction of the scan, a process that results in a parabolic profile shape even in the case of an ideally uniform beam. A qualitative comparison between simulation and experiment is possible though. In the case of the doped source measured profiles, the shapes of the integrated profiles indicate relatively uniform intensity beams. The relatively higher intensity in the center of the beam 
observed in some of the profiles is consistent with a case in which the emitter is retracted with respect to the Pierce electrode, thus provoking a faster drop of the profile at the edge. In the case of the aluminosilicate emitters there is a clear hollow character of the beam at the higher operating temperatures.

Table 4.1 presents a summary of the results obtained including the information on the beam brightness and equivalent ion temperature for the measured beams. The equivalent (or effective) ion temperature can be estimated from equation 4.7.

$$
k T=\left(\frac{\varepsilon_{n}}{2 r_{s}}\right)^{2} m c^{2}
$$

Here " $\mathrm{m}$ " is the mass of the ion, " $\mathrm{c}$ " is the speed of light and " $\mathrm{r}$ " is the radius of the emitter. The normalized brightness $\left(B_{n}\right)$ is given by equation 4.8 .

$$
B_{n}=\frac{2 I}{\pi^{2} \varepsilon_{n}{ }^{2}}
$$

\begin{tabular}{cccccc}
\hline & $\begin{array}{c}\text { Emitter } \\
\text { Temperature } \\
(\mathrm{C})\end{array}$ & $\begin{array}{c}\text { Total } \\
\text { Current } \\
(\mathrm{mA})\end{array}$ & $\begin{array}{c}\text { Normalized } \\
\text { Emittance } \\
(\pi . m m . m r a d)\end{array}$ & $\begin{array}{c}\text { Effective } \\
\text { Ion } \\
\text { Temperature } \\
(\mathrm{eV})\end{array}$ & $\begin{array}{c}\text { Normalized } \\
\text { Brightness } \\
\left(\mathrm{mA} /(\mathrm{mm} . \mathrm{mrad})^{2}\right.\end{array}$ \\
\hline K doped & 1,075 & 4.00 & $6.14 \mathrm{E}-02$ & 9.4 & 21.83 \\
source & 1,150 & 11.90 & $2.69 \mathrm{E}-02$ & 1.8 & 338.34 \\
& 1,205 & 12.44 & $3.44 \mathrm{E}-02$ & 3.0 & 216.28 \\
KalSi & 900 & 1.42 & $1.13 \mathrm{E}-01$ & 32.0 & 2.29 \\
source & 945 & 3.88 & $1.14 \mathrm{E}-01$ & 32.5 & 6.15 \\
& 990 & 6.30 & $7.32 \mathrm{E}-02$ & 13.4 & 24.21 \\
& 1,035 & 11.32 & $5.15 \mathrm{E}-02$ & 6.6 & 87.77 \\
& 1,075 & 15.60 & $5.88 \mathrm{E}-02$ & 8.7 & 92.69 \\
& 1,050 & 13.32 & $8.10 \mathrm{E}-02$ & 16.4 & 41.74 \\
& 1,100 & 17.04 & $5.48 \mathrm{E}-02$ & 7.5 & 116.72 \\
\hline
\end{tabular}

Table 4.1. Summary of emittance measurements including effective ion temperature and normalized brightness. 


\section{Chapter 5: Theoretical considerations.}

\subsection{The Theory of Contact Ionization Sources}

This chapter reviews the known theories about the physical principles that underlie the ion sources that have been analyzed in this work. The differences between the classical theory assumptions and the specific circumstances present in the emitters used throughout this dissertation are pointed out, hoping to elucidate the workings and potential of such sources in HIF research as well as the identified difficulties when operation in high current density regimes.

\subsubsection{Surface ionization.}

When an atom from a gas strikes a surface, the atom may become bound to the surface. This is known as adsorption. Further provision of energy to this atom may cause it to evaporate. Under certain conditions, the energetics of the system favors the transfer of an electron from the adsorbed atom to the surface, thus ionizing the particle. 
The basic condition for this phenomenon is that the ionization potential (I) of the adsorbate be lower than the surface's work function $(\phi)$. It is for these reasons that the systems used in surface ionization are limited to substrates with a high work function (tungsten, platinum, molybdenum) and adsorbates with low ionization potential (i.e.: the alkali species). The ionization is a probabilistic process, thus at any given time, in a population of adsorbed atoms there will be a fraction of the particles that has been ionized while the remainder fraction is left in its atomic, neutral state. The ratio of ionized particles to atoms in the adsorbed phase is given by the Saha-Langmuir equation:

$$
\frac{n_{p}}{n_{a}}=\frac{1}{2} \exp \left(\frac{e(\phi-I)}{k T}\right)
$$

where " $n_{p}$ " and " $n_{a}$ " are the number of ions and the number of atoms in the surface respectively. The term " $\mathrm{kT}$ " is the surface temperature in energy units as usual and " $\mathrm{e}$ " is the fundamental charge. For the species of interest in this work, Cs and $\mathrm{K}$, the ionization potential is respectively 3.87 and $4.3 \mathrm{~V}$. The work function for a clean tungsten substrate is $4.62 \mathrm{~V}$. Therefore, it is to be expected, at a typical operation temperature of $1,150{ }^{\circ} \mathrm{C}$ to have an ion to atom ratio of $\sim 225$. Equation 5.1 is valid whenever the surface concentration of the substrate is low enough as to leave the surface's work function unaffected, and when the presence of other adsorbates is not affecting (positively or negatively) the surface's $\phi$.

The measured values of current density compared to the measured values of neutral evaporation from the ion sources described in the previous chapter indicate the possibility that this ratio may have been enhanced due to an increase in the substrate's work function caused by the presence of oxygen. If true, this is one of the few instances 
in which uncontrolled factors actually affect the experimental outcome in a desirable fashion. An alternative and not exclusive explanation, which is the diffusion limited process that defines the coverage at the surface, will be also discussed below.

While equation 5.1 indicates that the ratio of ions on the surface to neutral particles is favored at lower temperatures, the actual evaporation of the particles is enhanced by increments in the surface temperature. It was for this reason that the ion gun design described in these pages required a heating module capable of achieving higher emitter temperatures than what is commonly used.

\subsubsection{Brief review of Langmuir's classical theory.}

The seminal and definitive work on the physics of desorption of alkali atoms from thin films on tungsten surfaces was elaborated by John B. Taylor and Irving Langmuir in their The Evaporation of Atoms, Ions and Electrons from Caesium Films on Tungsten (1933). Their work develops in wonderful detail the phenomenon of surface adsorption, focusing on the case of adsorbed films of cesium on tungsten. This section borrows extensively from Taylor and Langmuir's paper as the field was defined by their work, and their exposition of the various relevant phenomena is present in all discussions and publications of this subject.

The adsorbed atoms are held by large forces that change the surface's thermionic properties, such as electron emission and ionization adsorbed particles. Taylor and Langmuir determined quantitatively the dependence of the rates (in units of particles per unit time, per unit surface area) of neutral atom evaporation $\left(v_{a}\right)$, ion emission $\left(v_{p}\right)$ and electron emission $\left(v_{\mathrm{e}}\right)$ on the surface fractional coverage $(\theta)$ of the adsorbed films, 
surface temperature ( $\mathrm{T}$ ) and field strength. In order to achieve a correlation of the different emission rates with $\theta$, a significant part of their work consisted in establishing the functional dependence of $\theta$ on $\mathrm{Cs}$ pressure and filament temperature. All their analysis was made in conditions of steady state surface conditions, defined by the equation:

$$
\alpha \mu_{a}=v_{a}
$$

where $\alpha$ is the condensation coefficient (the fraction of the arriving particles that becomes adsorbed, shown by Langmuir to be equal to 1), and $\mu_{\mathrm{a}}$ is the particle arrival rate. Langmuir and Taylor proposed and justified the "surface phase postulate" that indicated that $v_{\mathrm{a}}, v_{\mathrm{p}}$, and $v_{\mathrm{e}}$, are functions of $\theta$ and $\mathrm{T}$ only, and that the surface distribution of atoms was assumed to be uniform and independent of the way in which $\theta$ was reached.

While conceptually simple, the actual measurement of the fractional surface coverage warrants more discussion. The tungsten surfaces under consideration in Langmuir's experiments, as well as the ones considered in HIF research, have an "apparent" area $\left(\mathrm{S}_{\mathrm{A}}\right)$ defined by the macroscopic geometry of the surface (although in HIF there is a further complication because of the surface porosity). However, after heating to 2,900 K, a tungsten surface becomes etched and develops dodecahedral crystal faces (110) in which there are $1.425 \cdot 10^{15}$ tungsten atoms $/ \mathrm{cm}^{2}$. The increased microscopic ruggedness of the etched surface makes the actual surface area (S) greater than the apparent area, with a ratio $\mathrm{S} / \mathrm{S}_{\mathrm{A}}$ being 1.347 , as measured by Taylor and Langmuir (see next paragraph). 
The crystals of metallic Cs have the same $\mathrm{BCC}$ arrangement as the tungsten, but with almost exactly double lattice constant $(6.17 \AA)$, thus making a ratio of 1 Cs atom per $4 \mathrm{~W}$ atoms in a complete monolayer. This implies that the true surface concentration $\left(\sigma_{1}\right)$ of a full film of Cs on $\mathrm{W}$ is $3.563 \cdot 10^{14}$ atoms $/ \mathrm{cm}^{2}$. However, experimentally the apparent surface concentration becomes $\sigma_{\mathrm{A} 1}=4.8 \cdot 10^{14}$ atoms $/ \mathrm{cm}^{2}$. Since the ratio $\mathrm{S} / \mathrm{S}_{\mathrm{A}}$ should be equal to $\sigma_{\mathrm{A} 1} / \sigma_{1}$, this immediately gave the value of the 1.347 factor. The parameter $\theta$ is defined as the number of atoms per unit of apparent surface, divided by the number of atoms in a complete layer of apparent surface:

$$
\theta \equiv \frac{\sigma_{A}}{\sigma_{A 1}}=\frac{\sigma}{\sigma_{1}}
$$

\subsubsection{Basic equation for the desorption of Cs from a tungsten surface.}

The data of Langmuir and Taylor's experiments can be summarized in functions for ion and atom desorption of the form:

$$
v=\exp \left(A-\frac{B}{T}\right)
$$

The form of the constants A and B vary for the two cases of atom (neutral) and ion desorption and are given by the expressions (Forrester, 1988):

$$
A_{a}=61+4.8\left(\theta-\frac{1}{2} \theta^{2}\right)+\ln \left(\frac{\theta}{1-\theta}\right)+\left(\frac{1}{1-\theta}\right)
$$

and

$$
B_{a}=\frac{32,380}{1+0.714 \theta}
$$

In the case of ion emission: 


$$
A_{p}=A_{a}-\ln 2
$$

and

$$
B_{p}=B_{a}-8,681+11,606 V_{c}
$$

where $V_{c}$ is the contact potential between a clean tungsten surface and a cesiated one. In other words, it is the amount by which Cs lowers the work function of the tungsten surface. The dependence of $\mathrm{Vc}$ on $\theta$ is given by:

$$
V_{c}=10.679 \theta-22.982 \theta^{2}+42.53 \theta^{3}-34.91 \theta^{4}
$$

A graphical representation of the atom and ion desorption rates is presented in Figure 5.1. This figure exemplifies the general features of the ion and atom desorption rates as a function of temperature and surface coverage. As expected in a case of evaporation, both rates increase with the surface temperature. The most important characteristic of the behavior of these curves is the way in which neutral paticle emission is favored at high coverages, while ion emission is favored at low coverages below $10 \%$. 


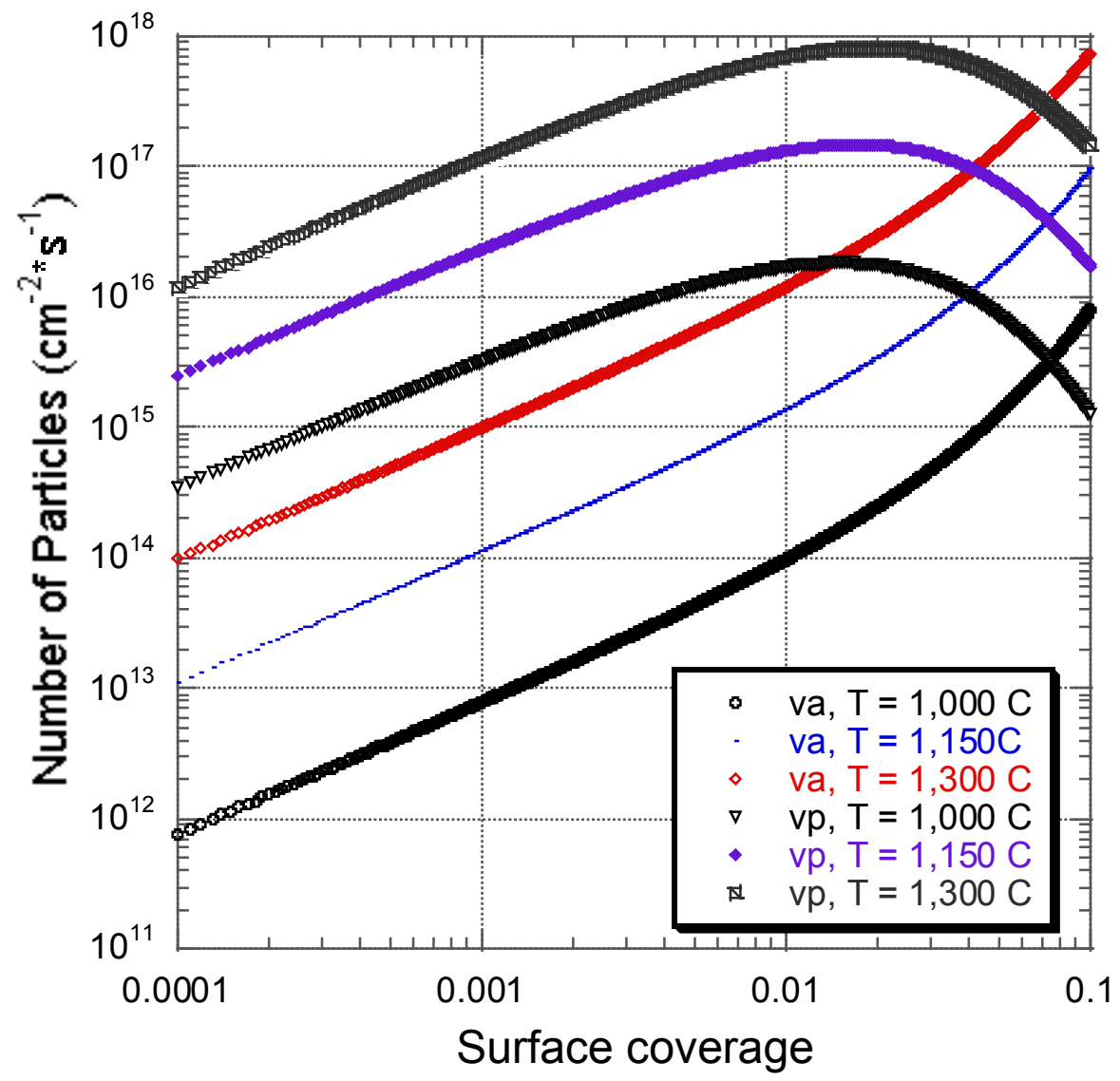

Figure 5.1. Atom $\left(v_{\mathrm{a}}\right)$ and ion $\left(v_{\mathrm{p}}\right)$ desorption rates for Cs on a tungsten surface as a function of surface coverage and temperature.

\subsection{On the desorption probability of a particle from a surface and the drooping of current pulses during the extraction pulse.}

It is convenient to continue the discussion of the theoretical aspects of ion and atom desorption from metallic substrates by addressing the problem of desorption probability. The original experiments that dealt with this problem (Evans, 1933) consisted of exploring the time constant for the decay of ion current pulses desorbed from tungsten upon sudden changes in the applied voltages of emitting and measuring electrodes. The results of these original experiments yielded a dependence of the decay time constant with temperature of the Arrhenius form: 


$$
\tau=\tau_{0} \exp \left(\frac{Q}{T}\right)
$$

where $\tau$ is the mean desorption time (or average residence time of the particle on the surface), $\tau_{0}$ is a proportionality constant, $\mathrm{T}$ is the surface temperature (in units of energy) and $\mathrm{Q}$ is the desorption energy of the species under consideration (atoms, ions or electrons). Figure 5.2 shows a diagram of a Born cycle for adsorption/desorption of Cs on a surface, with the terminology commonly used for these parameters.

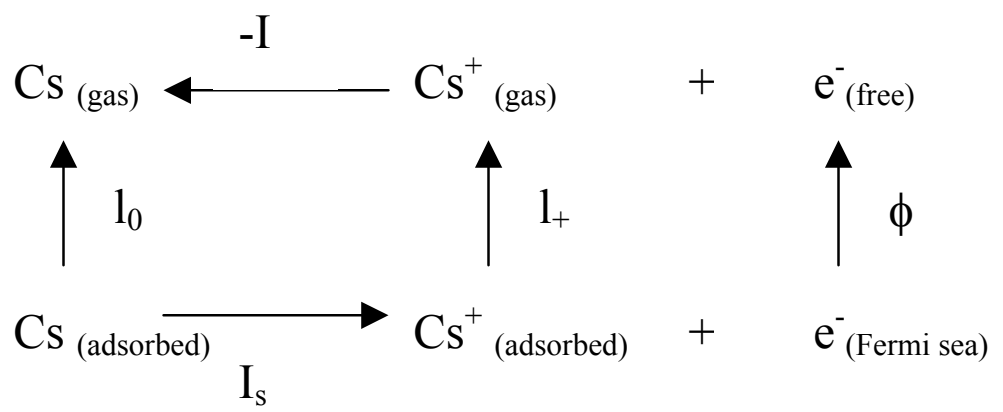

Figure 5.2 Diagram representing the nomenclature for the energetics of ion, atom and electron desorption from a surface, in the case of Cs.

Based on the above diagram, it is possible to work out the relationships between these parameters. For instance, the ionization energy of a Cs atom on the surface, in terms of the ionization energy of the free atom and the desorption energy of the ion and the electron from the surface, would be given by:

$$
I_{s}=\left(l_{0}-l_{+}\right)-(\phi-I)
$$

The literature is not in full agreement regarding the measured values for $\tau_{0}$ and the energy parameters, a feature that is understood in terms of the difficulty to attain sufficiently clean surfaces. Table 5.1 summarizes some of the Arrhenius desorption parameters for cesium ions on tungsten. 


\begin{tabular}{lrc}
\hline \multicolumn{1}{c}{ Experimenter and range of temperatures } & $\mathbf{I}_{+}(\mathbf{e V})$ & $\tau_{\mathbf{0}}(\mathbf{s})$ \\
\hline R. C. Evans $(935-1,010 \mathrm{~K})$ & 1.81 & $0.7 \cdot 10^{-10}$ \\
F. Knauer $(1,200-1440 \mathrm{~K})$ & 3.6 & $0.4 \cdot 10^{-16}$ \\
Scheer \& Fine, clean W $(1,070-1,200 \mathrm{~K})$ & 2.04 & $1.1 \cdot 10^{-12}$ \\
Scheer \& Fine, contaminated W (950-1,130 K) & 1.55 & $0.6 \cdot 10^{-10}$ \\
Langmuir (very low coverage approximation) & 2.04 & $1.13 \cdot 10^{-12}$
\end{tabular}

Table 5.1. Summary of time and energy desorption parameters for cesium ions on tungsten (From Scheer and Fine, 1962 and Langmuir, 1933).

The results presented in the above table must be taken with caution. The value of the desorption energy is dependent, albeit weakly for low alkali content, on the fractional surface coverage. Furthermore, the nature of the contaminants, which in most publications is treated as a wild card, may have either a positive or negative effect on the ion yield depending on whether the effective work function of the surface is increased or decreased. A contaminant like oxygen will increase the effective work function whereas a contaminant like carbon will decrease it. For these reasons, the values in the table should always be used as a guide and for making relatively rough estimates.

Regardless of these discrepancies, the measurements show that the mean lifetime of a cesium atom on a tungsten surface is extremely short. For instance, at an operating temperature of $1,100{ }^{\circ} \mathrm{C}$, using Scheer's values and with the reasonable assumption that the data can be extrapolated within such temperature range, the mean lifetime would be $\sim 18 \mu \mathrm{s}$. 
The desorption probability per unit time is given by $1 / \tau$. For a given surface concentration of particles in a surface, given by the product of the fractional coverage $(\theta)$ and the number of particles in a full monolayer $\left(\sigma_{1}\right)$, the ion desorption rate $\left(v_{\mathrm{p}}\right)$ would be approximated by

$$
v_{p}=\theta \sigma_{1} \frac{1}{\tau}
$$

A tacit assumption here is that the coverage is low, so that the fraction of the population that is composed of neutral atoms is very small. An equation of the form (5.12) will have a good agreement with the more general desorption equations described above when the coverage, $\theta$, is lower than 0.001 .

This equation is sufficient to model the time dependence of a current pulse when the surface coverage is sufficiently low to be affected by the current pulse itself. The continuity equation in the emitter surface has the form:

$$
\sigma_{1} \frac{d \theta}{d t}=-v_{p}=-\frac{J}{e}
$$

We have introduced $\mathrm{J}$, the current density, as the more common measured quantity. Taking the time derivative of equation (5.12), eliminating the $\mathrm{d} \theta / \mathrm{dt}$ term and integrating from $\mathrm{J}_{0}$ to $\mathrm{J}$ in the current density and from $\mathrm{t}_{0}$ to $\mathrm{t}$ in time, we arrive at:

$$
J=J_{0} \exp \left(-\frac{\left(t-t_{0}\right)}{\tau}\right)=J_{0} \exp \left(-\frac{\Delta t}{\tau}\right)
$$

The term $\Delta \mathrm{t}$ is the symbol for the current pulse length. This short analysis concludes that once the surface coverage in an emitter surface is low enough to be affected by the extracted current during the pulse length, the first sign of ion depletion should be a droop in the pulse with a characteristic exponential decay. The time constant for this decay is 
the mean residence lifetime of the alkali atom in the surface. A more general approach is given later in this section for the time dependence of $\mathrm{J}$ based on the classical theory of particle desorption for an arbitrary value of the initial coverage.

To illustrate a case of surface depletion during a long pulse test, there is some experimental data on a life time test done on a cesium doped contact ionization source. As said above, the experiment used the longest pulse length available in the experiments described in these pages, $10 \mu$ s. In reality the pulser was capable of delivering pulses up to $20 \mu \mathrm{s}$, however, given the impedance matching (or unmatching!) conditions, the risetime of the pulser was heavily affected, thus yielding a flat top of about $10 \mu$ s. Figure 5.3 shows the general time behavior at a fixed extraction voltage of this test.

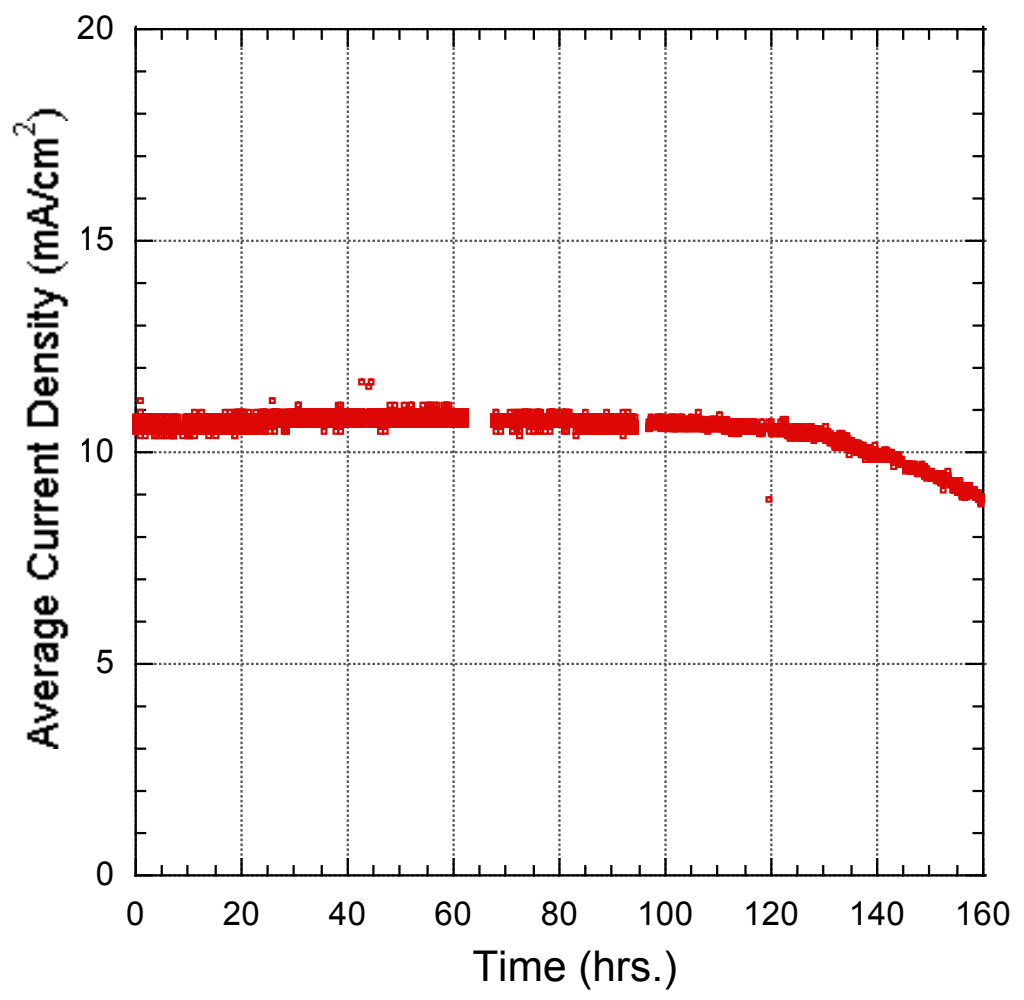

Figure 5.3 Life test of a cesium on tungsten source, at an operating temperature of 1,125 ${ }^{\circ} \mathrm{C}$. Current density measureents were made at the center of the pulse. 
A more detailed description of these and similar tests is presented in Chapter 4. Figure 5.3 presents the case where the ionizer was capable of delivering a steady value of the current over a period of time of tens of hours until a very distinct droop occurs during the pulse. Figure 5.4 shows the $\mathrm{J}-\mathrm{V}$ characteristics associated with this experiment, showing the decrease in output at the highest extraction voltages. Figure 5.5 shows a comparison of the $\mathrm{J}-\mathrm{V}$ characteristics obtained when considering the measurements at the beginning vs. the middle of the pulse.

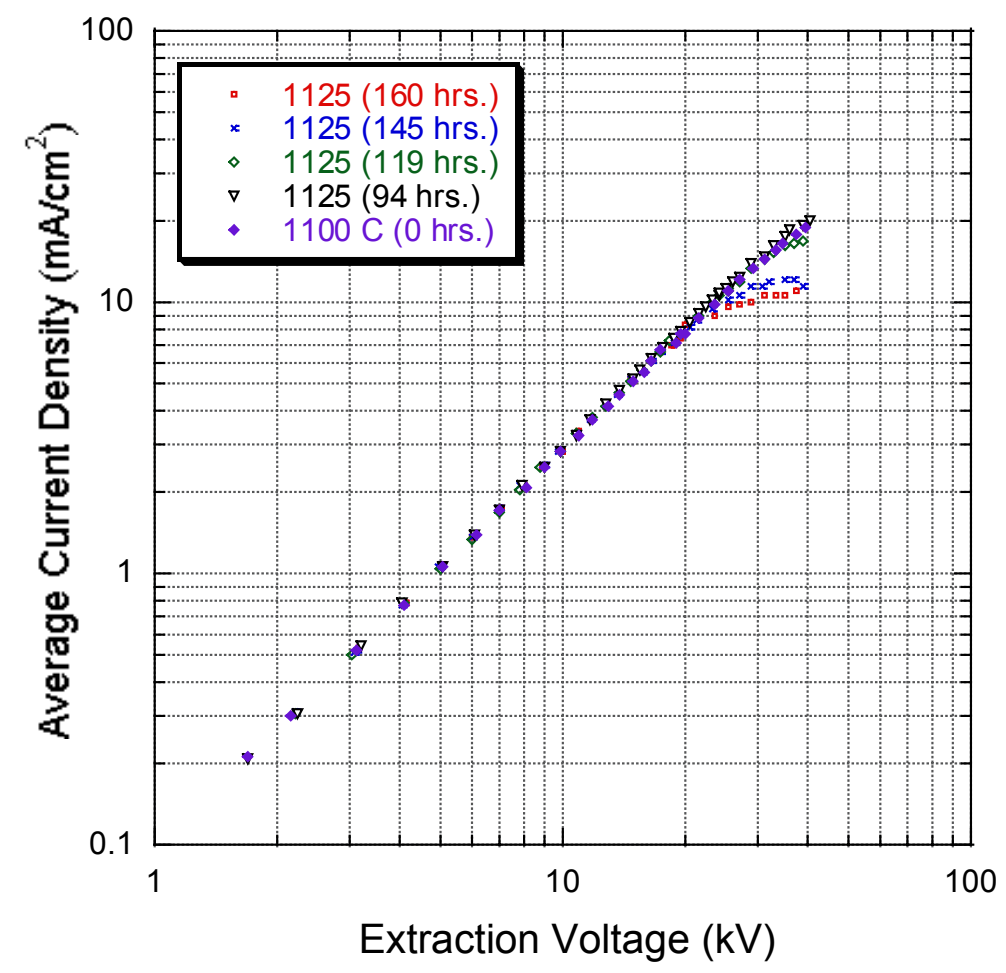

Figure 5.4 J - V characteristics of the ion source corresponding to different points in time of the test of Figure 5.2. 


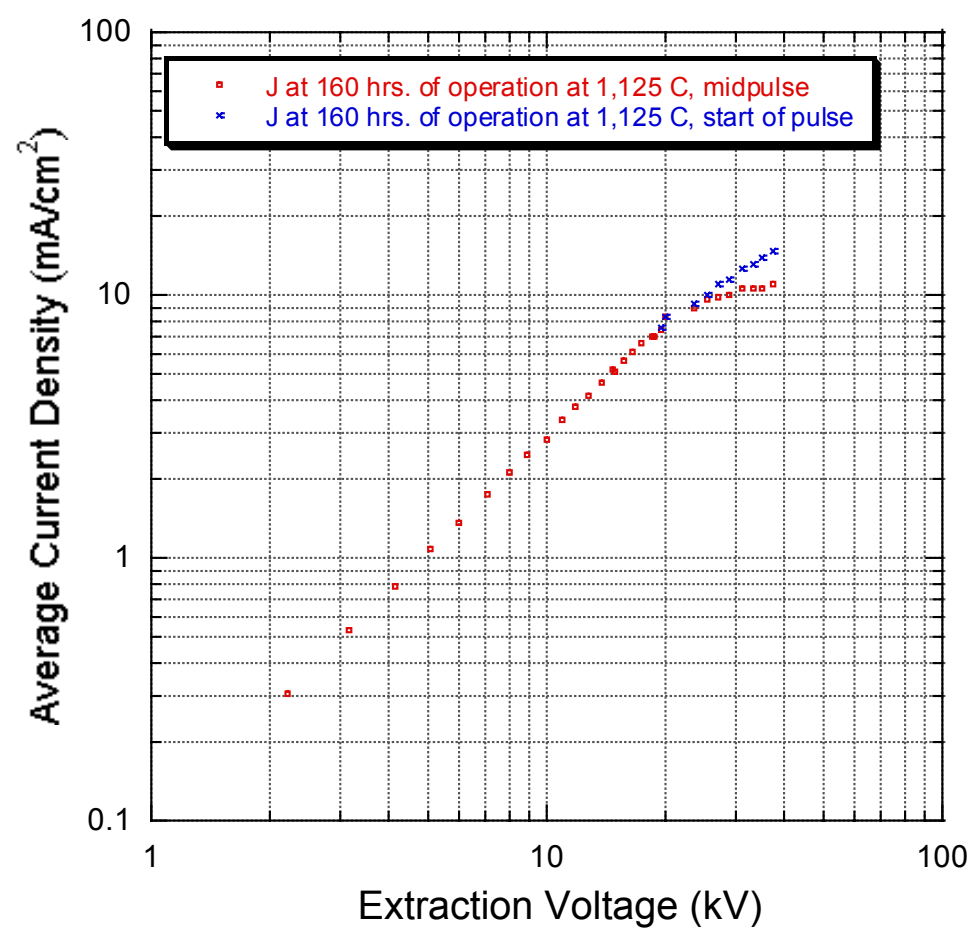

Figure 5.5 Comparison of the $\mathrm{J}-\mathrm{V}$ characteristics of the depleting source when measuring at the beginning versus the middle of the pulse.

Data regarding the current steadiness during the extraction pulse when the source was depleting is presented in Figures 5.6 through 5.11. The first figure of the series presents the high voltage pulse as well as a typical current pulse for reference purposes. The remaining figures present the drooping current during the pulse length. The photographs of the different scope traces were taken at different extraction voltages, thus showing the more pronounced effects of current decay at different current density demands. It can be seen how upon increasing the voltage, the current droop is more significant, indicating the increase of the extent to which the surface coverage is being affected by the current pulse. 


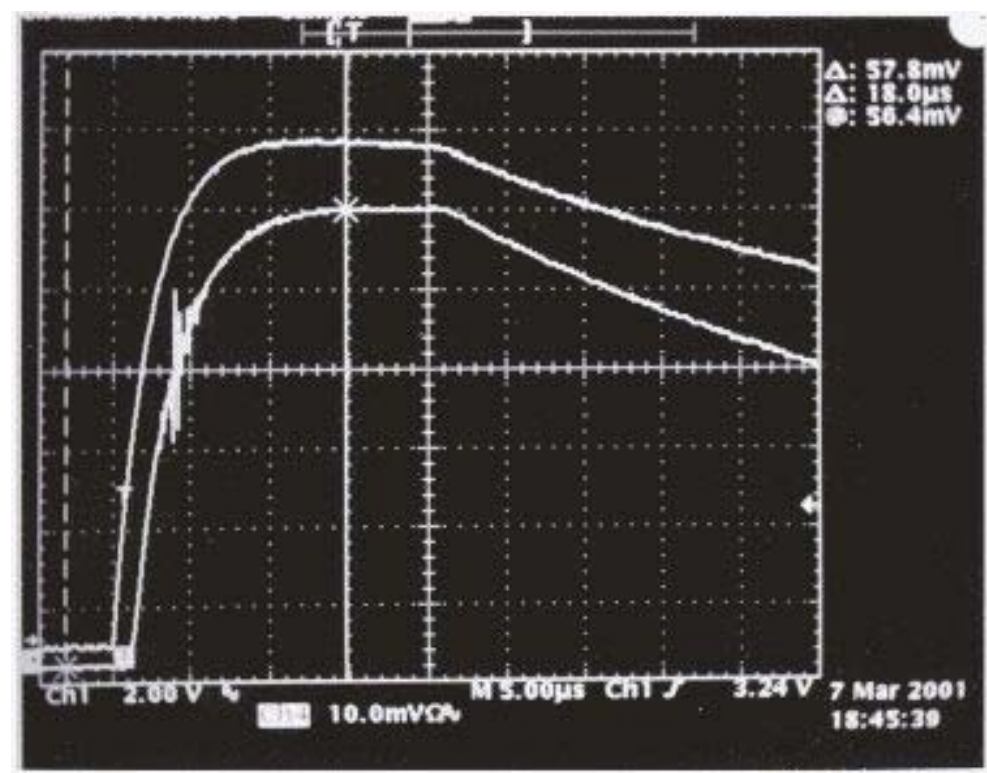

Figure 5.6. Flat reference pulse of the Cs beam mentioned above, during normal operation. Upper trace is the High Voltage pulse.

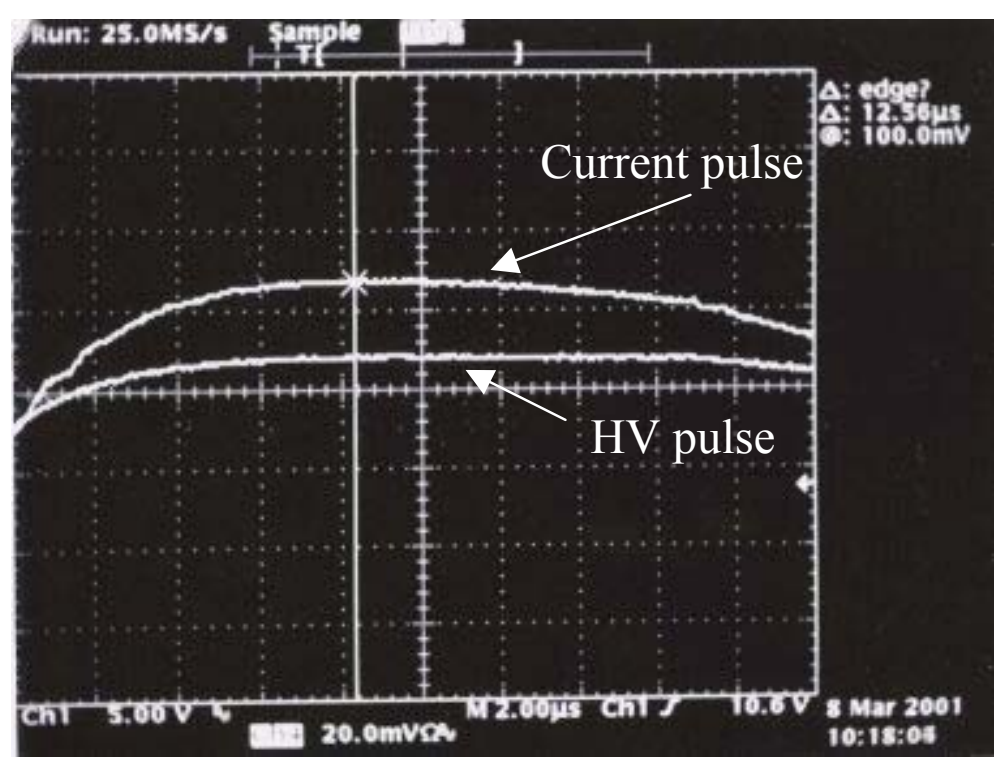

Figure 5.7. Current pulse shape at an extraction voltage of $19.5 \mathrm{kV}$, after $160 \mathrm{hrs}$. of operation at $1,125^{\circ} \mathrm{C}$. 


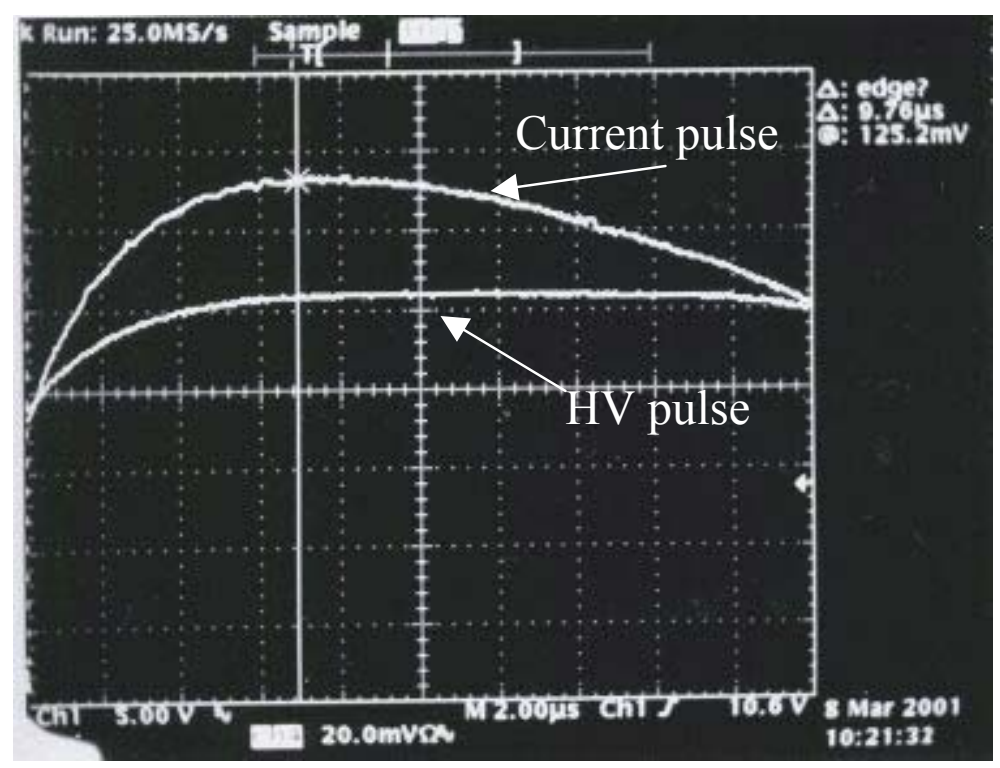

Figure 5.8. Current pulse shape at an extraction voltage of $23.5 \mathrm{kV}$, after $160 \mathrm{hrs}$. of operation at $1,125^{\circ} \mathrm{C}$.

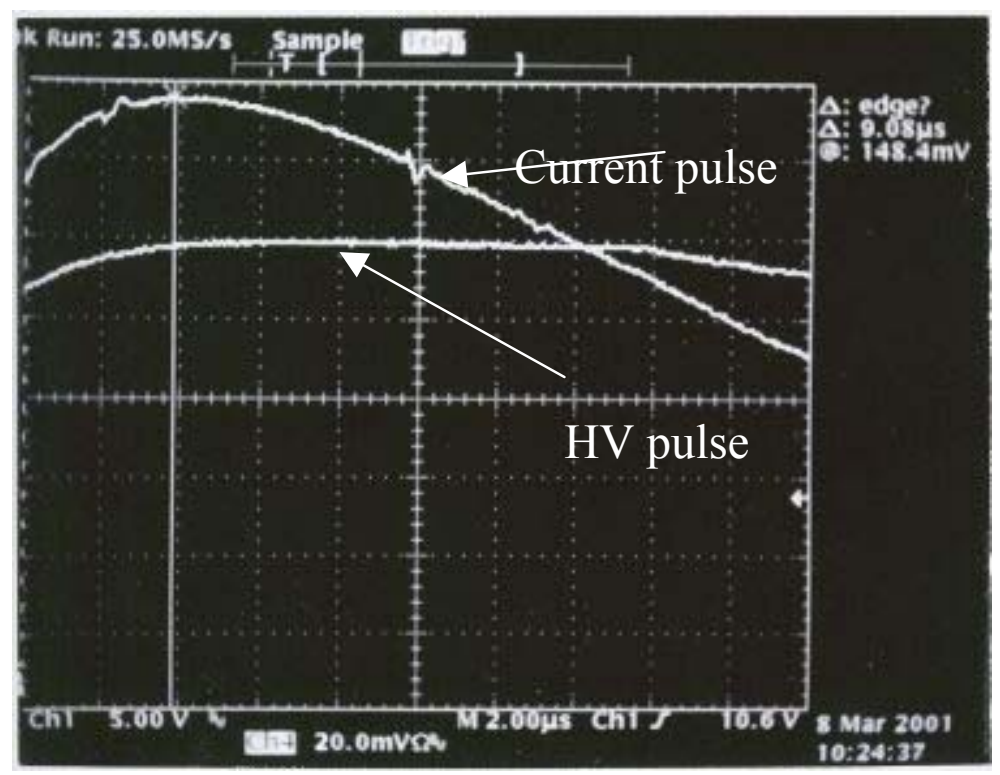

Figure 5.9. Current pulse shape at an extraction voltage of $27.3 \mathrm{kV}$, after $160 \mathrm{hrs}$. of operation at $1,125^{\circ} \mathrm{C}$. 


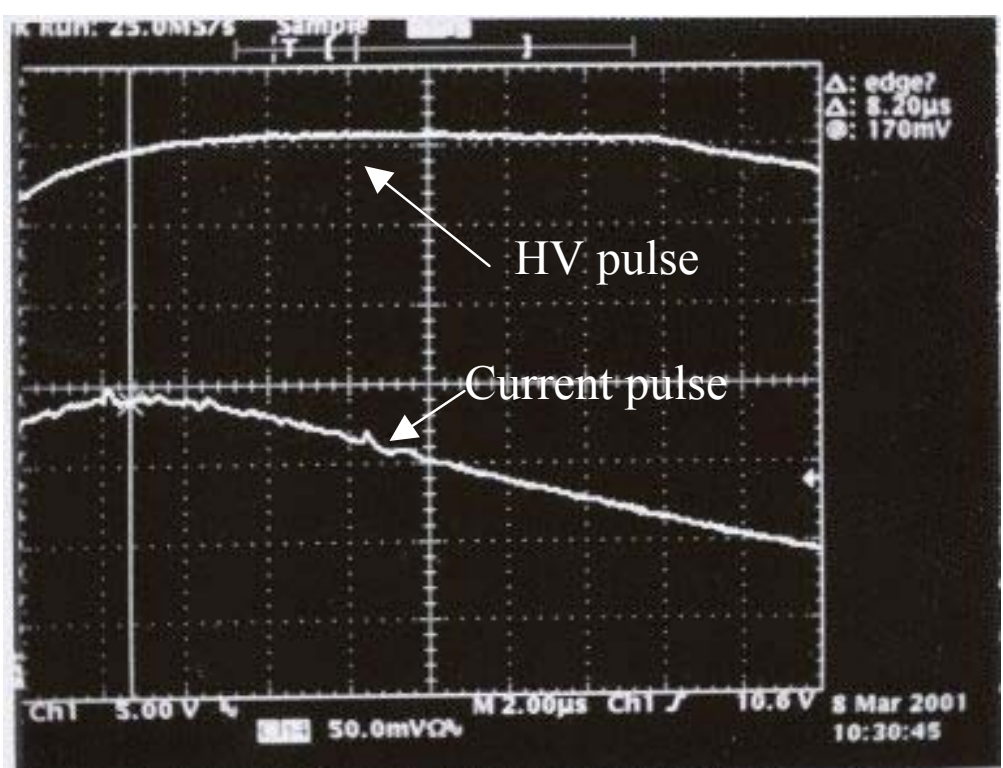

Figure 5.10. Current pulse shape at an extraction voltage of $33.1 \mathrm{kV}$, after $160 \mathrm{hrs}$. of operation at $1,125^{\circ} \mathrm{C}$.

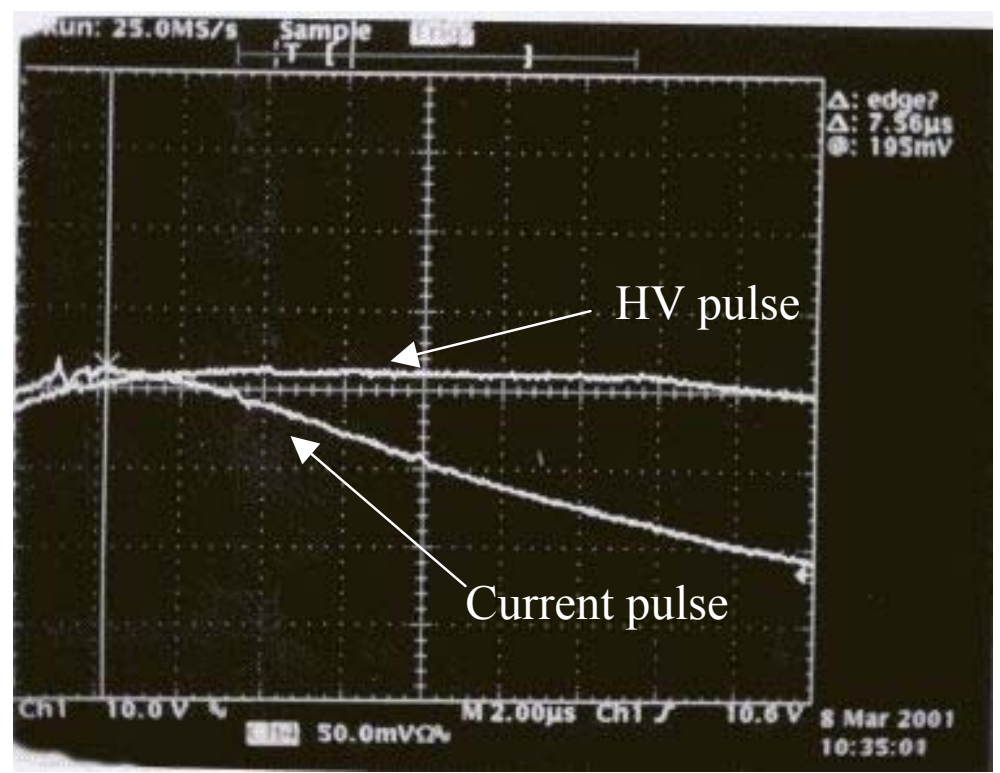

Figure 5.11. Current pulse shape at an extraction voltage of $37.4 \mathrm{kV}$, after $160 \mathrm{hrs}$. of operation at $1,125^{\circ} \mathrm{C}$.

Table 5.2 summarizes the information that is presented in the previous figures. The table was prepared by taking an interval within the high voltage pulse and measuring the ratio of the current pulse at the beginning and at the end of the interval. The theoretical ratio column is the expected value of the droop when the surface conditions 
change as required by the model described earlier in Equation 5.14. The values for a contaminated surface have been chosen for this comparison and are from Scheer and Fine, in Table 5.1.

\begin{tabular}{cccc}
\hline $\begin{array}{c}\text { Extraction Voltage } \\
(\mathrm{kV})\end{array}$ & $\begin{array}{c}\text { Time interval } \\
(\mu \mathrm{s})\end{array}$ & $\begin{array}{c}\text { Ratio of currents on } \\
\text { interval }\left(\mathrm{J}_{2} / \mathrm{J}_{1}\right)\end{array}$ & Theoretical ratio \\
\hline 19.5 & 9.6 & 0.96 & 0.66 \\
23.5 & 11.2 & 0.82 & 0.61 \\
27.3 & 10 & 0.71 & 0.65 \\
33.1 & 10 & 0.56 & 0.65 \\
37.4 & 12 & 0.51 & 0.59 \\
\hline
\end{tabular}

Table 5.2. Comparison of measured droop in a depleted Cs source with the predicted droop based on a simple model. The model gives more realistic results at higher operation currents.

At this point it is not clear how the information on the drooping pulses may provide some kind of insight into the approximate value of the coverage present at the emitter surface. An exponential rate of decay is predicted by the simple model shown above, but as it as also mentioned, the accuracy of such model is limited to very low coverages, namely, values below $\theta=0.001$. This may explain the discrepancies observed in the last table.

The previous discussion leads immediately to the question: If there is such dependence between the current density "J" and the surface coverage, why is a droop in the current pulse something not inherent to this type of source, in any pulse? The answer would clearly depend on what is the fractional change of the surface coverage that occurs during the extraction pulse. In other words, if the coverage is sufficiently large, or the demanded current sufficiently small for the surface coverage to remain approximately unaffected during the extracted pulse, then no effects of pulse drooping should be observed. While the previous analysis holds for very low surface coverages in which the 
extracted current is expected to have a significant impact on the surface coverage itself, it is also possible to extend this analysis using the general desorption laws discovered by Langmuir presented earlier. The idea behind this effort is that it is possible in principle to assess the approximate values of surface coverage in the ion emitter by examining the properties of the time-evolution of the current pulses when in operation in the emission limited mode. This is shown next.

In Langmuir's classical experiments, all time dependence effects were effectively eliminated by ensuring conditions of steady state in terms of achieving a balance between the rate of deposition of particles on the surface, and the atom and ion desorption rates. By ensuring constant values of surface coverage by the alkali atoms and ions, Langmuir was able to establish accurately the dependence of the desorption parameters with respect to only two variables, the surface coverage $(\theta)$ and the surface temperature (T). Langmuir was able to ensure the constancy of the surface coverage through a strict control of the pressure of the Cs gas atmosphere, thus controlling the rate of deposition of atoms on the surface. This could carefully be equilibrated with the rates of desorption under examination in his experiment.

The surface conditions existing in HIF sources are very different than Langmuir's. While it is expected that the functional relation between the ion and atom desorption rates with respect to $\theta$ and $\mathrm{T}$ will still hold, the careful control of the surface coverage in HIF's ion sources is non existent. The performance of the ion sources is completely determined by the total ion content, the diffusion rates through the porous tungsten (as it will be explained below), and the quasi-steady state conditions achieved at the surface of the emitter. This quasi-equilibrium condition is due to the delicate equilibrium between the 
rates of neutral particle desorption, the ion and atom diffusive replenishment rate and the occasional ion desorption pulse.

The term "quasi-equilibrium" is used since the surface conditions (the effective alkali coverage) change slowly with time. However during fast transient conditions occurring during current extraction, the surface coverage may be affected in an important way.

The expected decay of the current pulse when the coverage is low has been previously shown. In such conditions, drooping pulses are expected. However, in the case of long pulses and/or high current density demands, it is important to assess the expected changes, if any, in the behavior of the current pulse due to changes in the surface alkali coverage.

In order to transform the steady state equations (5.4) and subsequent into a time dependent estimate, the most straightforward way is to treat the problem by successive iterations. That is, for a given temperature and initial value of the surface coverage, the initial value of the ion desorption rate $\left(v_{p}\right)$ will be determined. Then the surface coverage $(\theta)$ will be in turn affected by the desorbing particles, since the diffusive replenishment rate is not significant during the current pulse time scale. This yields a new value of $\theta$ given by

$$
\theta_{i}=\theta_{i-1}-\frac{v_{p}\left(\theta_{i-1}, T\right)}{\sigma_{1}}\left(t_{i}-t_{i-1}\right)
$$

where $\theta_{\mathrm{i}}$ is the initial value of the coverage in the " $\mathrm{i}-\mathrm{th}$ " time interval and $\left(\mathrm{t}_{\mathrm{i}}-\mathrm{t}_{\mathrm{i}-1}\right)$ represents the time step used for each calculation. A general value of $0.1 \mu$ s was used throughout this analysis. The successive estimation of $v_{p}(\theta, T)$, based on a changing $\theta(t)$ 
(due in turn to $\operatorname{vp}(\theta, \mathrm{T})$ ), effectively yields the sought for time dependence of the extracted current density.

The previous methodology was used then to estimate the variation of the current density with time with the initial value of the surface coverage as a parameter. Figure 5.12 shows a family of these calculations with different values of initial coverage, at the same temperature of operation $\left(1,125{ }^{\circ} \mathrm{C}\right)$ as the cesium ion source described in the previous pages.

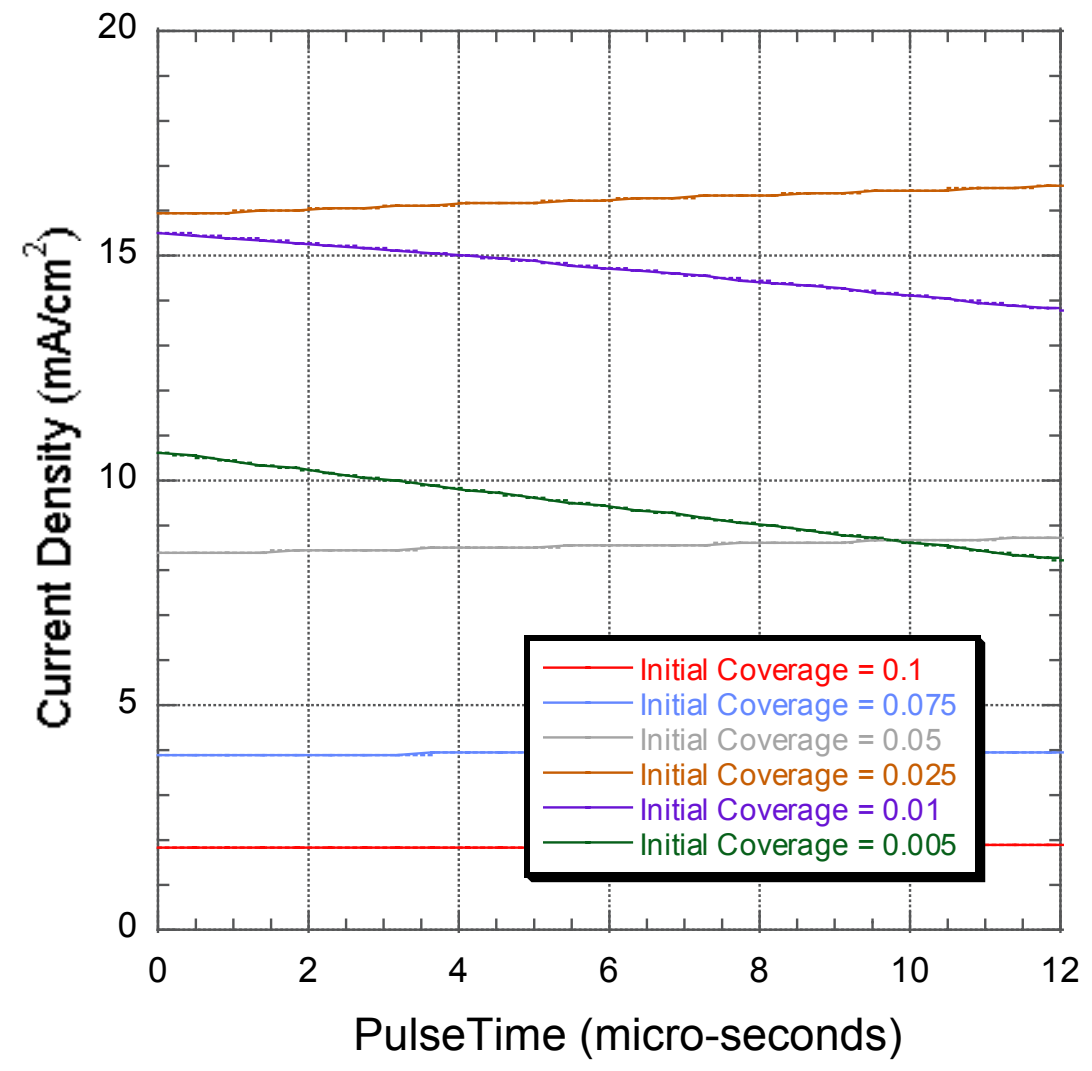

Figure 5.12. Expected time evolution of the current density level of a cesium on tungsten doped emitter, operating in the emission limited mode, with the initial Cs surface coverage level used as a parameter. Source temperature: $1,125^{\circ} \mathrm{C}$.

The curves obtained are very informative in several ways. First, the curves denote significant variations of the output current density on the same scale as the pulse lengths of interest for HIF. This shows that whenever there is no fast replenishment of 
alkali atoms on the surface, the demanded current density levels are sufficient to significantly affect the surface coverage within the pulse time. Second, the type of variation of the current pulse may be a good indication of the approximate level of surface coverage existing at the emitter. If the surface coverage is higher than the value that yields the peak current density value, then the current pulse will further decrease the surface coverage towards this optimum value, thus increasing the current output during the pulse. On the other hand, if the coverage is equal to or lower than the optimum value, then the additional decrease in surface coverage will cause a reduction of the ion current with time.

Inspection of Figure 5.12 may show that qualitatively, the emitter should be operating with a surface coverage lower than $0.5 \%$ in order to explain the observed magnitude of the droop in the current pulse. The discrepancy in the initial value of the current density at such coverage may further be qualitatively explained by the uncertainties in the temperature measurement. In this case, the emitter surface may have a higher temperature than the measured value, in order to deliver the slightly higher current density levels.

The interest in the level of influence of the current pulse on the surface coverage is sufficiently large to justify presenting here the more general cases of this phenomenon at different temperatures. Figures 5.13 through 5.18 show the expected time dependence of the current pulses of a cesium on tungsten source, at operating temperatures of 1,050 , $1,100,1,150,1,200,1,250$ and $1,300{ }^{\circ} \mathrm{C}$ respectively. The time chosen for the analysis has been $45 \mu \mathrm{s}$. 


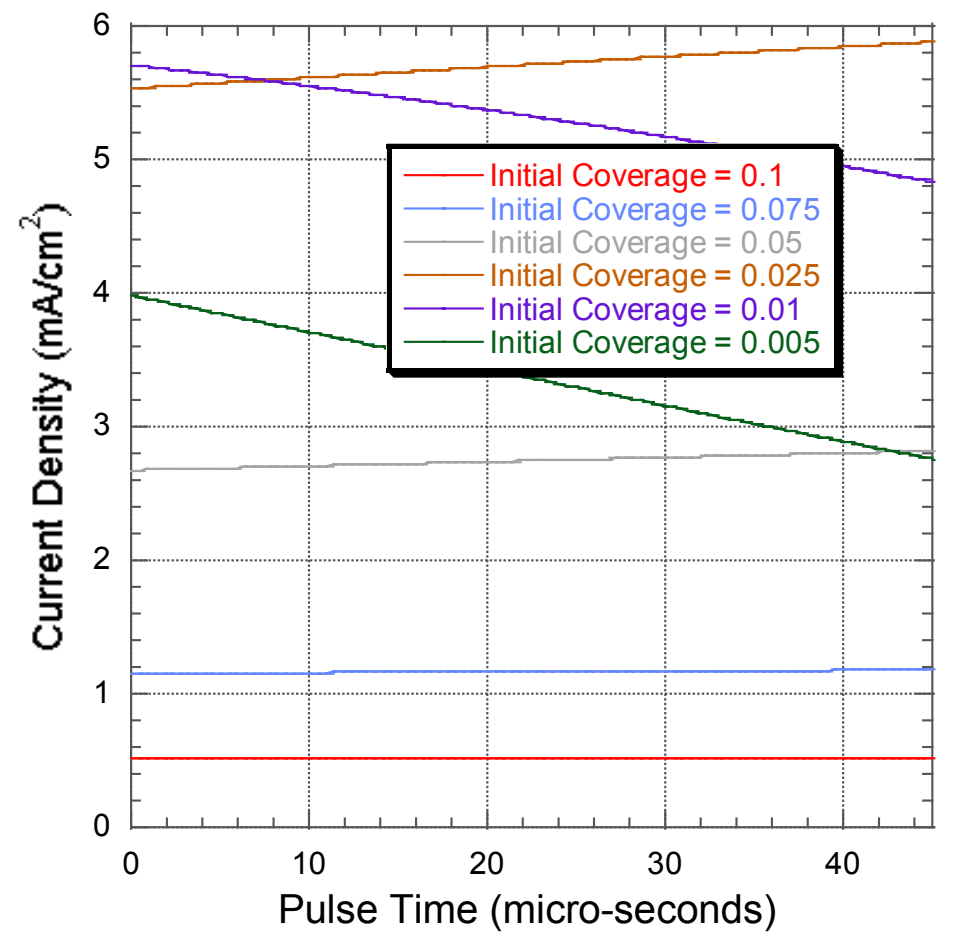

Figure 5.13. Time evolution of the delivered current density by a cesium contact ionization source, showing the effect on the delivered current due to the changes in the surface alkali content provoked by the current pulse. Temperature $=1,050^{\circ} \mathrm{C}$.

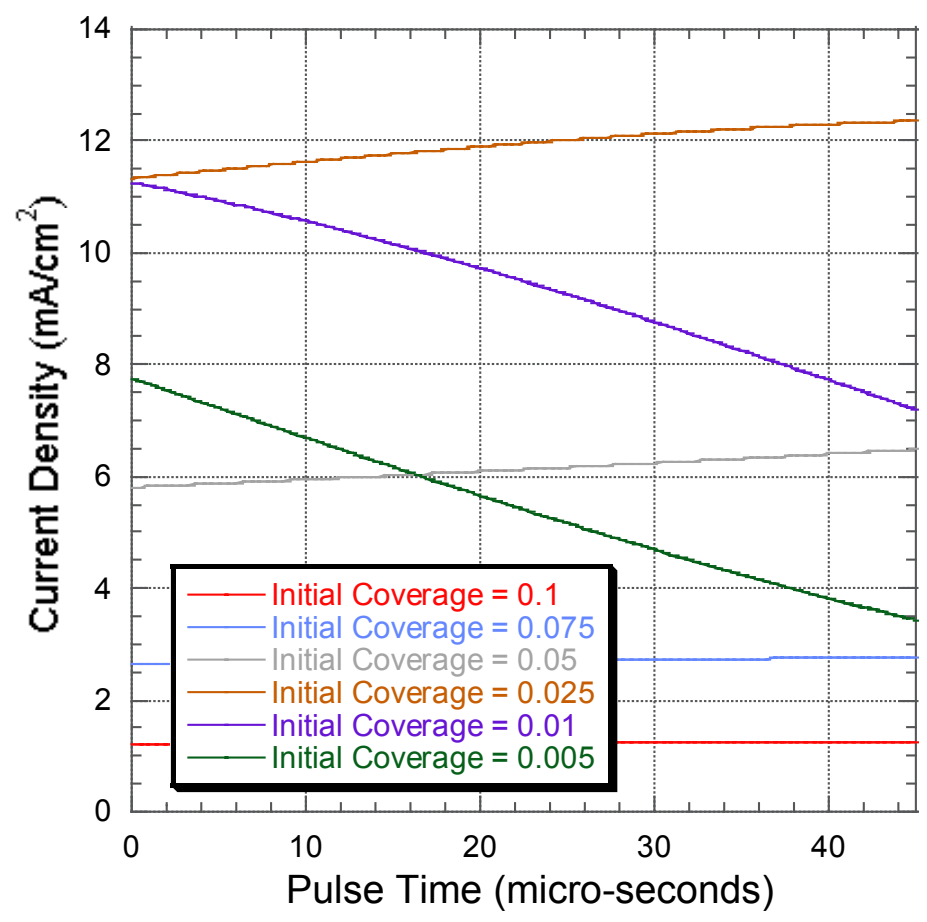

Figure 5.14. Time evolution of the delivered current density by a cesium contact ionization source, showing the effect on the delivered current due to the changes in the surface alkali content provoked by the current pulse. Temperature $=1,100^{\circ} \mathrm{C}$. 


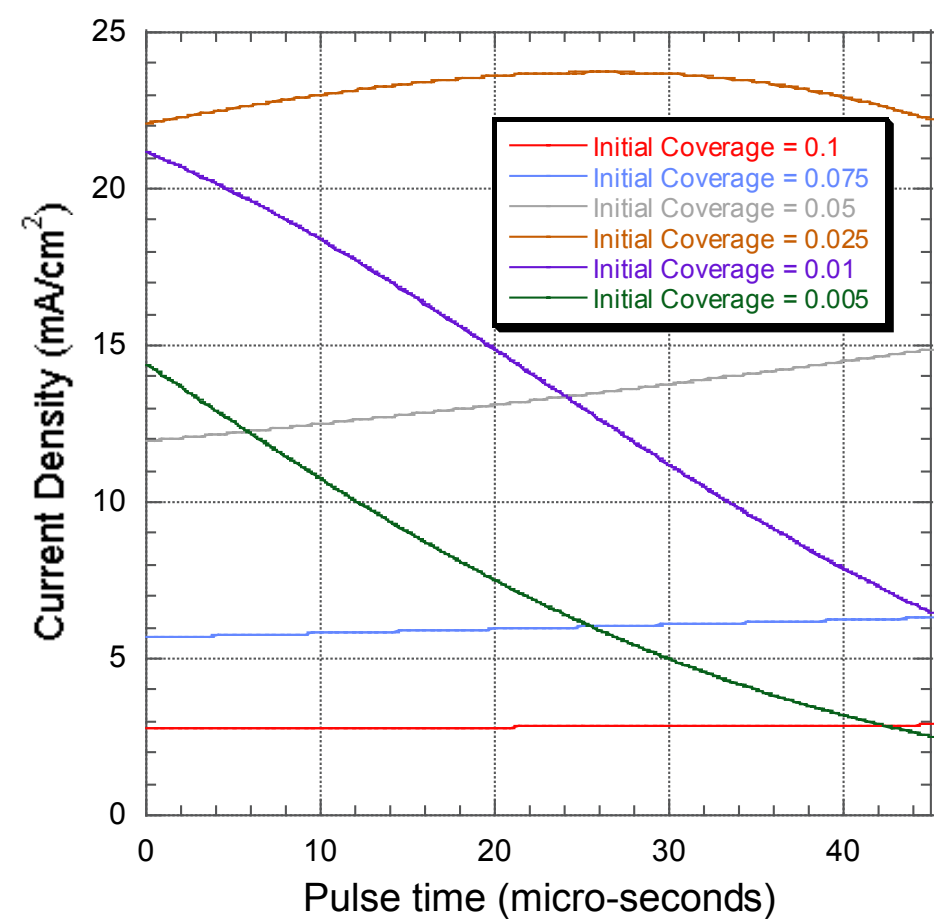

Figure 5.15. Time evolution of the delivered current density by a cesium contact ionization source, showing the effect on the delivered current due to the changes in the surface alkali content provoked by the current pulse. Temperature $=1,150^{\circ} \mathrm{C}$.

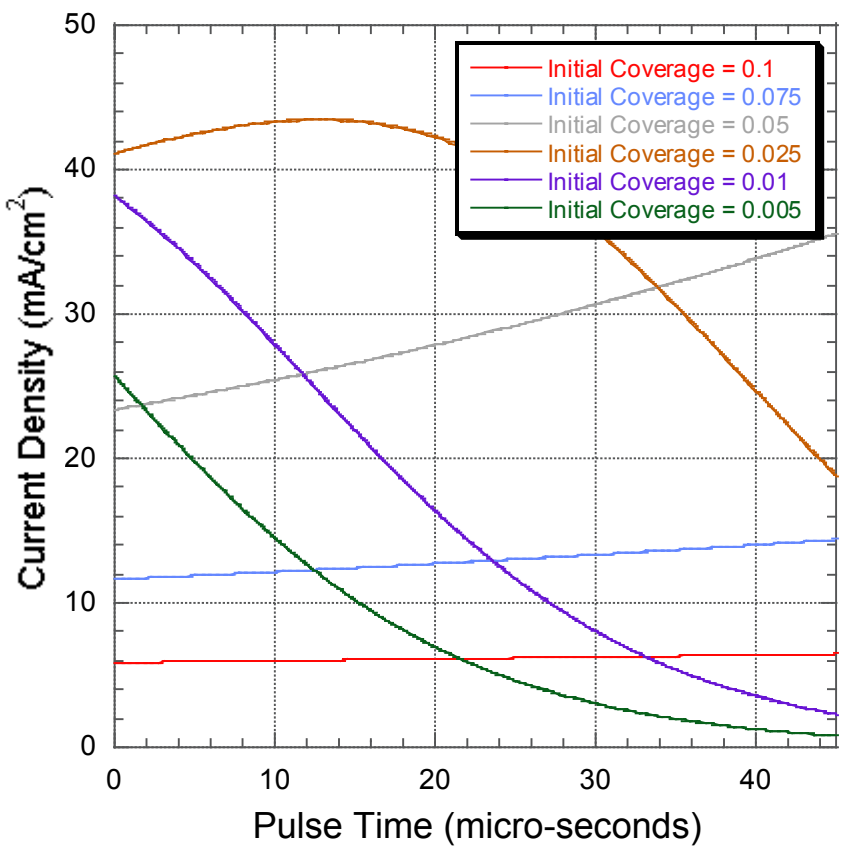

Figure 5.16. Time evolution of the delivered current density by a cesium contact ionization source, showing the effect on the delivered current due to the changes in the surface alkali content provoked by the current pulse. Temperature $=1,200^{\circ} \mathrm{C}$. 


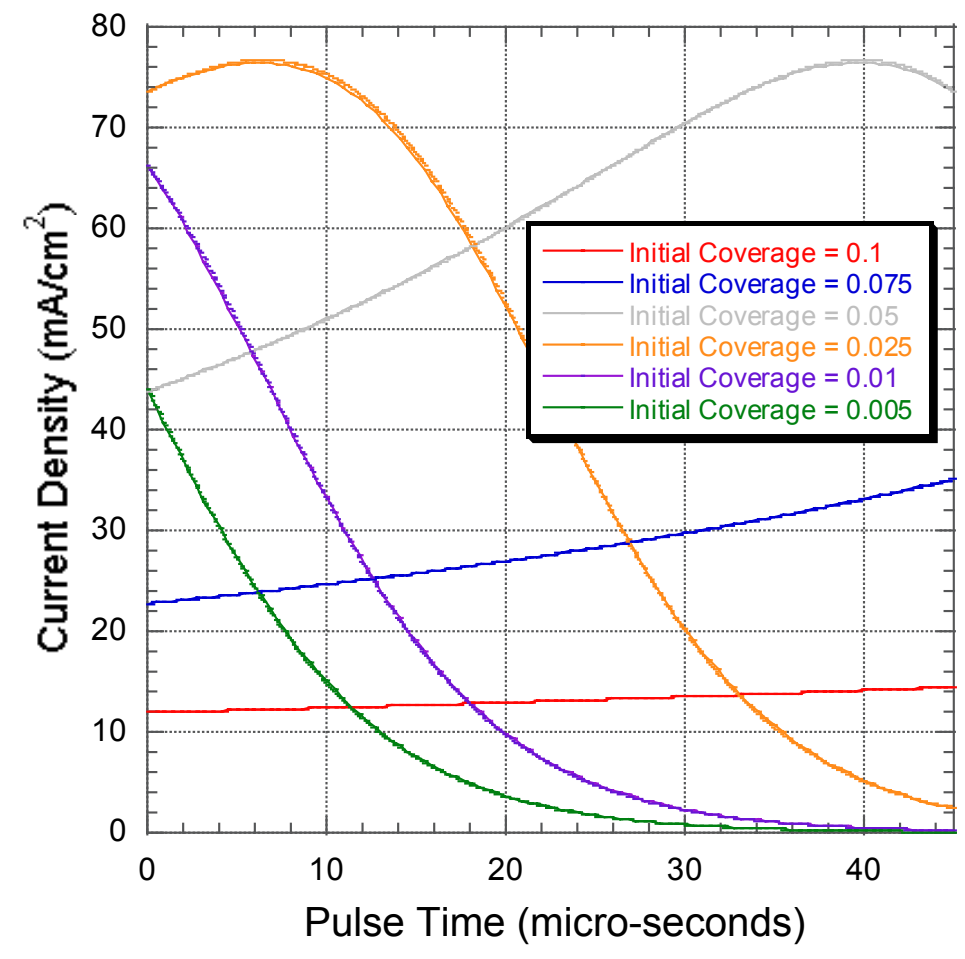

Figure 5.17. Time evolution of the delivered current density by a cesium contact ionization source, showing the effect on the delivered current due to the changes in the surface alkali content provoked by the current pulse. Temperature $=1,250^{\circ} \mathrm{C}$.

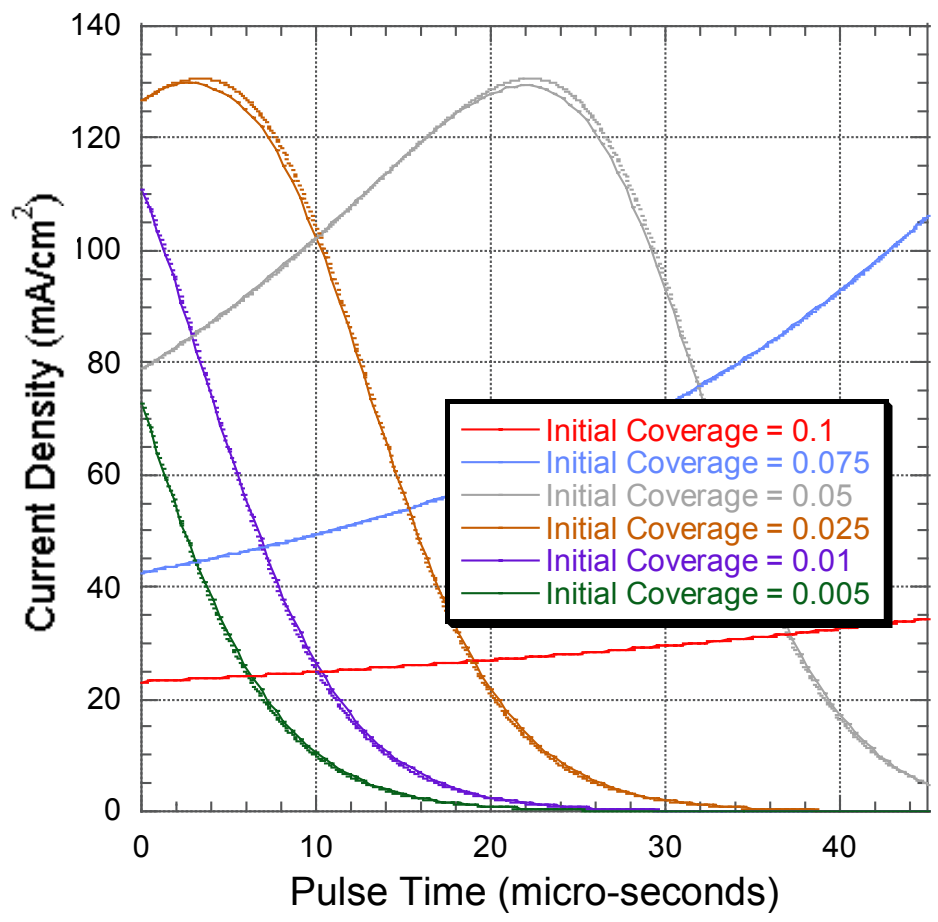

Figure 5.18. Time evolution of the delivered current density by a cesium contact ionization source, showing the effect on the delivered current due to the changes in the surface alkali content provoked by the current pulse. Temperature $=1,300{ }^{\circ} \mathrm{C}$. 


\subsection{On the lifetime of a contact ionization source.}

An immediate question that arises after the discussion presented in the previous section is: if the ions can live for such a short time on the surface of the ionizer, why is the source lifetime measured in tens of hours? The short answer to this question is that only a small fraction of the atoms or ions in the emitter actually live on the surface of the emitter at any given time. The lifetime of the ion source is then mostly governed by the diffusion rate of the two existing species (neutral atoms and ions) from the emitter bulk towards the surface. This process occurs on a longer time scale than the desorption of the particles from the surface, providing a slow replenishment, sufficient to keep the observed levels of current density. This section will try to address the general issues and present relevant experimental data when available.

\subsubsection{On the diffusion of the alkali species towards the emitter surface.}

The topic of diffusion deserves special attention since it is the most important process in the understanding of the performance and behavior of the surface ionization sources studied in this work.

The cylindrical emitter is initially doped with a diluted solution of an alkali carbonate as described in Chapter 4. Let us consider the case of potassium carbonate and its decomposition process as described by the chemical equations (5.16), (5.17) and (5.18).

$$
\begin{gathered}
\mathrm{K}_{2} \mathrm{CO}_{3} \rightarrow \mathrm{K}_{2} \mathrm{O}+\mathrm{CO}_{2} \\
\mathrm{~K}_{2} \mathrm{O} \rightarrow 1 / 2 \mathrm{~K}_{2} \mathrm{O}_{2}+\mathrm{K} \\
2 \mathrm{~K}_{2} \mathrm{O}_{2} \rightarrow 2 \mathrm{~K}_{2} \mathrm{O}+\mathrm{O}_{2}
\end{gathered}
$$


These reactions occur at temperatures of $600{ }^{\circ} \mathrm{C}, 300{ }^{\circ} \mathrm{C}$ and $600{ }^{\circ} \mathrm{C}$ respectively. Upon decomposition of the carbonate, $\mathrm{CO}_{2}$ escapes the emitter by diffusion while the $\mathrm{K}_{2} \mathrm{O}$ immediately decomposes into $\mathrm{K}_{2} \mathrm{O}_{2}$ and atomic potassium. It is not clear yet what fraction if any of the oxygen produced by the combined reactions (5.17) and (5.18) remains within the porous tungsten emitter. In experiments carried out with a residual gas analyzer (RGA) in LBNL's 2 MV injector, the expected oxygen signal has failed to show up, indicating that despite the reactions that produce it, the oxygen has not been released in the vacuum tank's atmosphere. It is for this reason that the increased ion to neutral ratio observed in our experiments can be at least partially explained by an increased work function caused by the oxygen presence in the tungsten surface.

Even when considering the case of the diffusion of a single species, the process is highly dynamical and somewhat complicated. Initially, the concentration of potassium or cesium in the emitter is relatively large, given the dose of carbonate used, which provides enough alkali atoms to have a full monolayer of coverage throughout the internal surfaces of the emitter. It is expected that the initial distribution of the alkali atoms will become more uniform upon the breaking down of the chemical compounds and the natural diffusion of the alkalis in the bulk. Several stages can be identified:

a. An initial stage of an alkali rich bulk. This stage should be identified at temperatures beyond $600{ }^{\circ} \mathrm{C}$. At this stage, the high concentration of alkali atoms favors their existence as neutral particles which have a smaller surface affinity with the tungsten than the alkali ions. Therefore, a gas neutral particle dominated 
process is expected. This does not occur at very fast rates since this stage usually happens while the emitter is at relatively low temperatures.

b. A mid-stage where sufficient alkali atoms have been desorbed so that there starts to appear a significant ion population. The neutral atoms will continue to diffuse through like a gas, being slowed down by the fact that the gas atoms do not elastically collide with the emitter internal surfaces and bounce right off, but become adsorbed, spend some time on the surface and then become desorbed again. The ions, on the other hand, due to their binding force to the surface, are limited to a slower diffusion speed, as their movement is through surface migration along the grain surfaces.

c. A final stage where the alkali content is sufficiently low to favor the existence of ions, which as said in point b., move by surface diffusion. It is at this stage that the source is fully operational and capable of its higher current yields. It is also this slow surface diffusion mechanism that explains the observed lifetimes of the surface ionization sources.

At this point it becomes very desirable to have a model that will describe the relation between the concentration of the alkali atoms at the emitter surface and the diffusion of the species in the bulk characterized by its diffusion coefficient "D". Since the cylindrical emitter has only one surface where the alkali atoms can actually leave the bulk (the other surfaces are covered by the tight molybdenum holder, which is flush with the front surface of the emitter), the problem can be understood in terms of a onedimensional diffusion problem. The analytical solution of this problem becomes difficult given the nature of the boundary condition at the emitter surface, which acts almost like a 
perfect absorber of the alkali atoms. An alternative method to attack this problem may be a one-dimensional particle-in-cell simulation, which could describe the surface concentration of the alkali atoms, and relate the time dependence of this concentration to the diffusion constant. Knowledge of this dependence would allow for a measurement of the diffusion constant of the species in the bulk at different operating conditions, since the surface concentration, or more precisely, the neutral evaporation rate that directly relates with the surface concentration, has already been measured as a function of time (see Figure 4.57).

A third method was found and applied on the following analyses. Based on the observations:

1. The migration of alkali atoms from the bulk towards the surface of the emitter is a diffusion process.

2. The neutrals can be evaporated from the surface sufficiently fast so that at any given time the surface concentration (and neutral desorption) will reflect the arrival rate of alkalis from the bulk.

3. The diffusion can be treated as a one-dimensional problem.

4. The time scale for the diffusive process makes the emitter behave like a thick body, so that the semi-infinite body approximation common in the solution of diffusive processes is appropriate.

Following the methodology for the type of problem mentioned in point 4., the problem may be imagined as a thick slab with a uniform particle concentration. The dimensions are measured from the mid-plane of the slab $(\mathrm{x}=0)$, and only one side of this 
slab is taken into account for the analysis. What is sought for is a solution of the diffusion equation (5.19), subject to the initial and boundary conditions (5.20) and (5.21).

a)

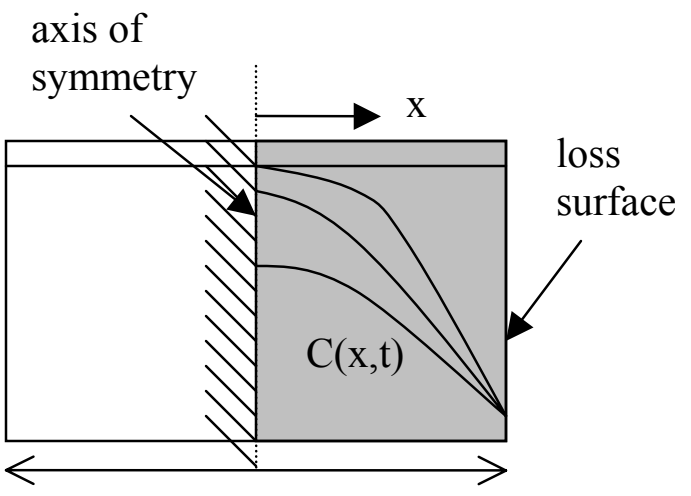

$2 \mathbf{L}$

b)

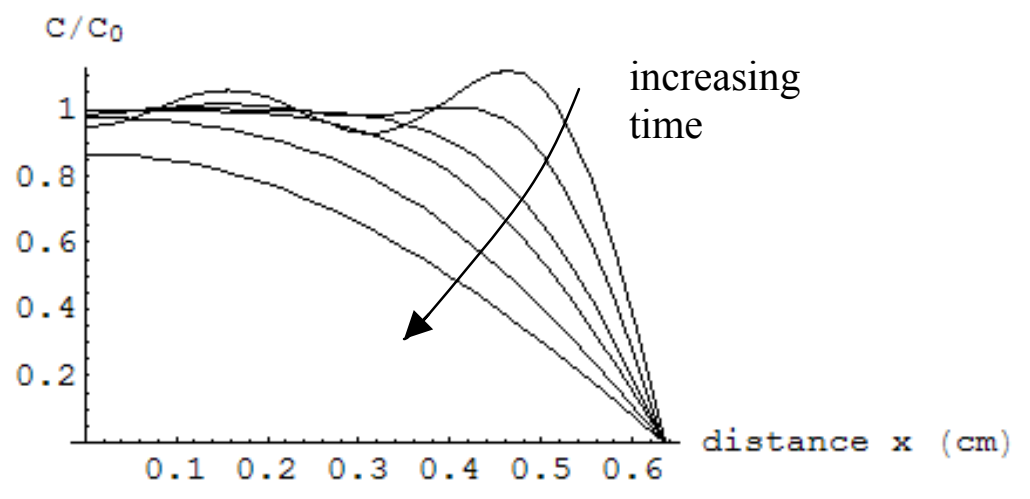

Figure 5.19. a) Schematic of the diffusion problem solved to model the migration of the alkali atoms through the porous tungsten substrate. b) Plot of the actual solution (described in the text below) within the emitter region, with $\mathrm{D}=2 \cdot 10^{-6} \mathrm{~cm}^{2} / \mathrm{s}$, and $\mathrm{t}=500$; 2,$000 ; 5,000 ; 8,000 ; 16,000 ; 30,000$ seconds.

$$
\begin{gathered}
\frac{\partial c}{\partial t}=D \frac{\partial^{2} c}{\partial x^{2}} \\
c(x, 0)=c_{0} \\
\left.\frac{\partial c}{\partial x}\right|_{x=0}=0
\end{gathered}
$$


The second boundary condition at the surface of the emitter $(x=L)$ must reflect the condition that the desorption rate of the particles is very high. The emitter surface effectively "absorbs" the particles that reach it. How this boundary condition is handled will become more clear later when the analogy with an equivalent heat transfer problem is discussed.

The solution to this problem is exactly known as it is commonly used in transient heat conduction problems. In the present context, the problem then becomes only to adapt the known solution to the conditions found in the measurements. In the heat transfer problem, equation (5.19) is maintained except that "c" is replaced by " $\mathrm{T}$ ", then the solution is given by the following expressions in heat transfer nomenclature (Incropera, 2002).

$$
\frac{T-T_{\infty}}{T_{i}-T_{\infty}}=\sum_{n=1}^{\infty} C_{n} \exp \left(-\frac{\varsigma_{n}^{2} D t}{L^{2}}\right) \cos \left(\frac{\varsigma_{n} x}{L}\right)
$$

Here $\mathrm{T}$ is the temperature in the slab at a given position and time, $\mathrm{T}_{\infty}$ is the ambient temperature with which the surface of the slab exchanges heat by convection and $T_{i}$ is the initial uniform temperature of the slab. The coefficients $C_{n}$ are given by the following expression:

$$
C_{n}=\frac{4 \sin \left(\varsigma_{n}\right)}{2 \varsigma_{n}+\sin \left(2 \varsigma_{n}\right)}
$$

where the values of the $\zeta_{n}$ 's are the successive positive solutions to the trascendental equation:

$$
\varsigma_{n} \tan \left(\varsigma_{n}\right)=\frac{h L}{k}
$$


where finally, $\mathrm{h}$ is the convective heat transfer coefficient and $\mathrm{k}$ is the heat conduction coefficient of the slab. The right hand side of equation (5.24) is known as the Biot number and is a nondimensional parameter indicating the relative strength of the rates of conduction of heat from the surface by convection to the internal heat diffusion in the material.

Adapting the solution to the conditions in our experiment, since at a given temperature the surface of the emitter is presumably capable of emitting all the neutral particles it receives from the substrate by diffusive transport, then the extreme case where the ratio " $\mathrm{hL} / \mathrm{k}$ " tends to infinity has been chosen. This seems reasonable given that the diffusion rate towards the surface is much slower than the desorption process. This choice defines the following values for the first four terms $C_{n}$ and $\zeta_{n}$. These values are presented in the following table.

\begin{tabular}{crr}
\hline $\mathbf{n}$ & \multicolumn{1}{c}{$\boldsymbol{\zeta}_{\mathbf{n}}$} & \multicolumn{1}{c}{$\mathbf{C}_{\mathbf{n}}$} \\
\hline 1 & 1.5708 & 1.27324 \\
2 & 4.7124 & -0.42441 \\
3 & 7.854 & 0.254648 \\
4 & 10.9956 & -0.18189 \\
\hline
\end{tabular}

Table 5.3. Values for the first four terms of the series of equation (5.13).

Continuing with the adaptation of the solution to the particular features of our problem, the cosine term in equation (5.22) plays the role of a normalizing factor. Specifically, given its dependence on the Biot parameter (through its dependence on $\zeta_{\mathrm{n}}$ ) it decreases the ratio on the left hand side of equation (5.22) if the convective heat transfer rate is large, basically equalizing the surface temperature with the ambient temperature. The cosine term also assigns a particular relative weight to the different terms of the 
series. For the purposes of our problem, the first role of the cosine term was ignored, because a normalized solution with an initial value of 1 is desired. Nevertheless, the second role of the cosine term was kept, by assigning coefficients to the different terms of the series that preserve the original relative weight of the elements in the solution series. This choice of values has no influence on the desired estimate of the diffusion coefficient. It is very important to note that this normalization is made only to ease the matching of the experimental data of evaporation rates with the surface concentration. If the analytical model is used to estimate the concentration changes within the bulk, then the original expression, without dividing by the first cosine term, must be used.

The left hand side of equation (5.22) is replaced by the ratio of the surface concentration (or what is actually measured, the neutral desorption rate), to its initial value in the interval of interest, "c/ $\mathrm{c}_{0}$ ".

By adapting the solution in the ways mentioned above, it is possible to make a fit to the experimental data by setting the initial value of the function to match the neutral desorption rate, and then having only a single parameter, the diffusion coefficient, to match the behavior of the experimental data. The final result, the formula that has been used throughout for the estimates of the diffusion coefficient is then given by equation (5.25). 


$$
\begin{aligned}
& \frac{C}{C_{0}}=1.27324 \exp \left(-\frac{1.5708^{2} D t}{L^{2}}\right) \frac{\cos \left(\frac{1.5708 x}{L}\right)}{\cos \left(\frac{1.5708 x}{L}\right)}-0.42441 \exp \left(-\frac{4.7124^{2} D t}{L^{2}}\right) \frac{\cos \left(\frac{4.7124 x}{L}\right)}{\cos \left(\frac{1.5708 x}{L}\right)}+ \\
& 0.254468 \exp \left(-\frac{7.854^{2} D t}{L^{2}}\right) \frac{\cos \left(\frac{7.854 x}{L}\right)}{\cos \left(\frac{1.5708 x}{L}\right)}-\left.0.18189 \exp \left(-\frac{10.9956^{2} D t}{L^{2}}\right) \frac{\cos \left(\frac{10.9956 x}{L}\right)}{\cos \left(\frac{1.5708 x}{L}\right)}\right|_{x=L=0.635}
\end{aligned}
$$

The desorption occurs at the surface of the emitter, hence $\mathrm{x}=\mathrm{L}=0.635 \mathrm{~cm}$. It is known that a good approximate solution for values of $\mathrm{Dt} / \mathrm{L}^{2}>0.2$ is given by using only the first term in the solution series. In our case a four term series is used. The following figures show some of the fits that have been made in the case of the cesium neutral desorption data. Figure 5.20 shows the measured data at $680{ }^{\circ} \mathrm{C}$ with the fitted curve generated with the series expansion. The nature of the fit gives confidence that the simple model captures the essential phenomena occurring in the bulk of the emitter.

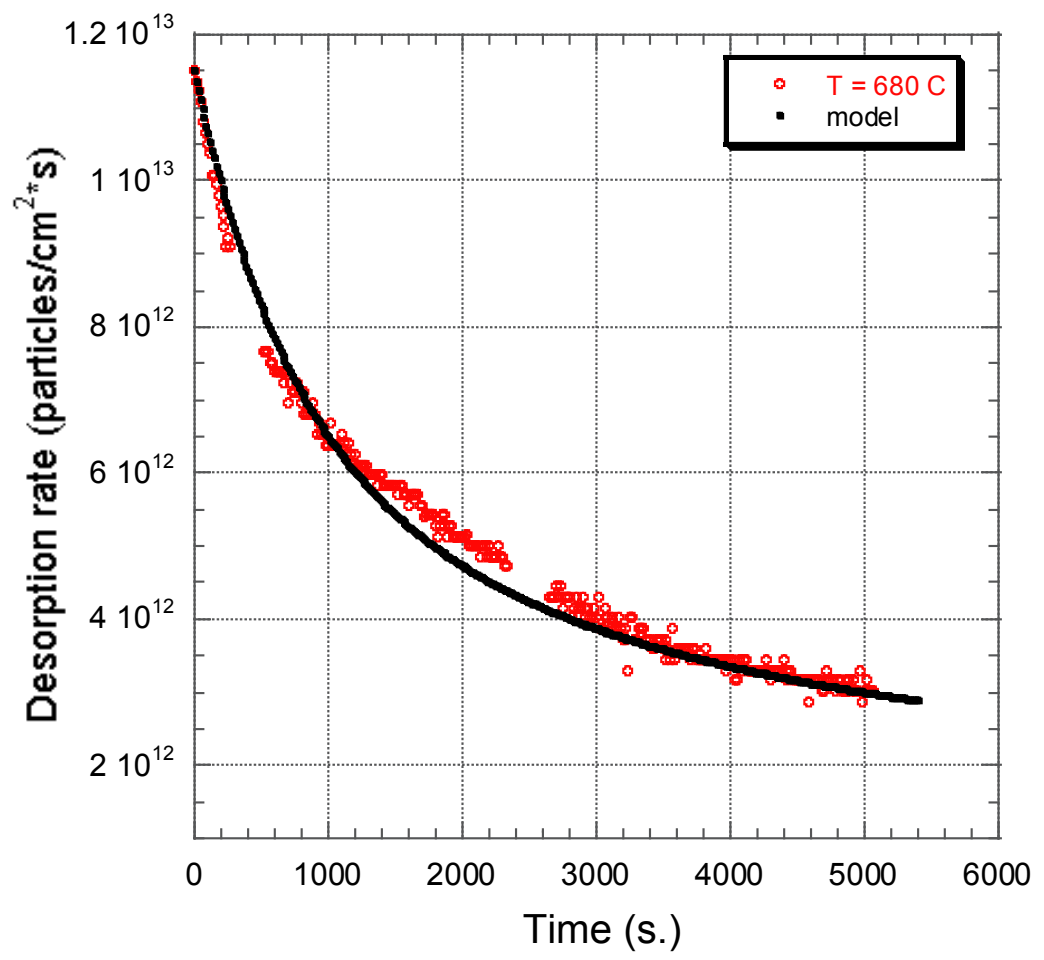

Figure 5.20. Fitting of the Cs neutral desorption data at a temperature of $680{ }^{\circ} \mathrm{C}$. Diffusion constant: $5.9 \cdot 10^{-6} \mathrm{~cm}^{2} / \mathrm{s}$ 
It is possible to relate this diffusion constant to what would be expected based on microscopic considerations. The diffusion constant would have the form

$$
D=\frac{(\text { stepsize })^{2}}{\tau}
$$

where as usual $\tau$ represents the mean surface residence time of the adsorbed particle. Assuming that the average step size is half the diameter of a tungsten grain $(\sim 1 / 2 \cdot 12 \mu \mathrm{m}=$ $6 \mu \mathrm{m})$, the average residence time at this temperature, would be $\sim 61$ milliseconds.

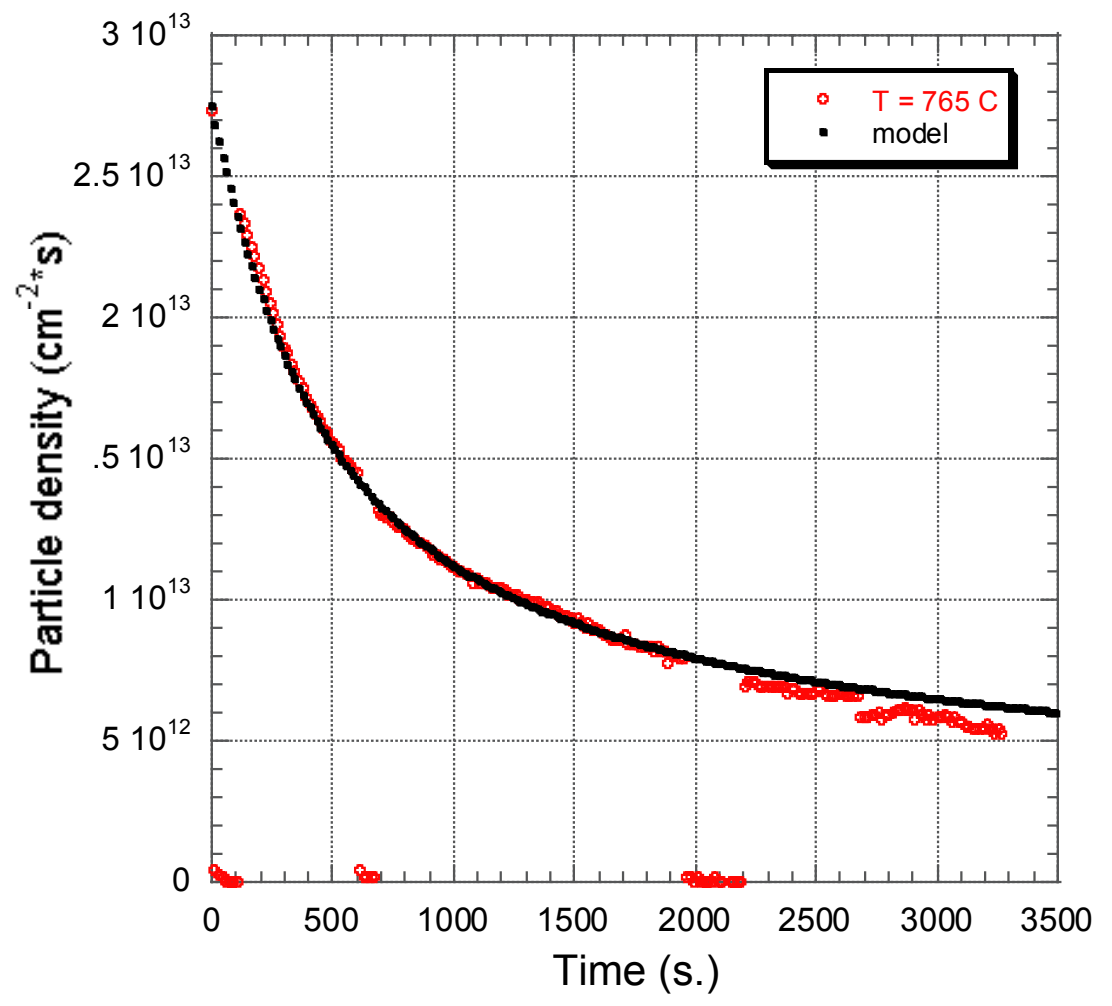

Figure 5.21. Fitting of the Cs neutral desorption data at a temperature of $765^{\circ} \mathrm{C}$ with equation 5.15. Diffusion constant: $1.2 \cdot 10^{-5} \mathrm{~cm}^{2} / \mathrm{s}$ 
Figures 5.22 through 5.26 show different fits at diverse temperatures for the data curves gathered on cesium. If a single species were diffusing, one would expect a behavior of the form:

$$
D \propto \exp \left(\frac{\varepsilon}{k T}\right)
$$

where $\varepsilon$ is some characteristic energy barrier for the desorption process that allows the diffusion of the particles. In our case this parameter would be an effective energy that would combine the desorption energy of the migrating atoms and ions. Unfortunately, the increasing fraction of particles migrating as ions with time, which to some extent opposes the trend of increasing the diffusion coefficient, does not allow for this simpler behavior.

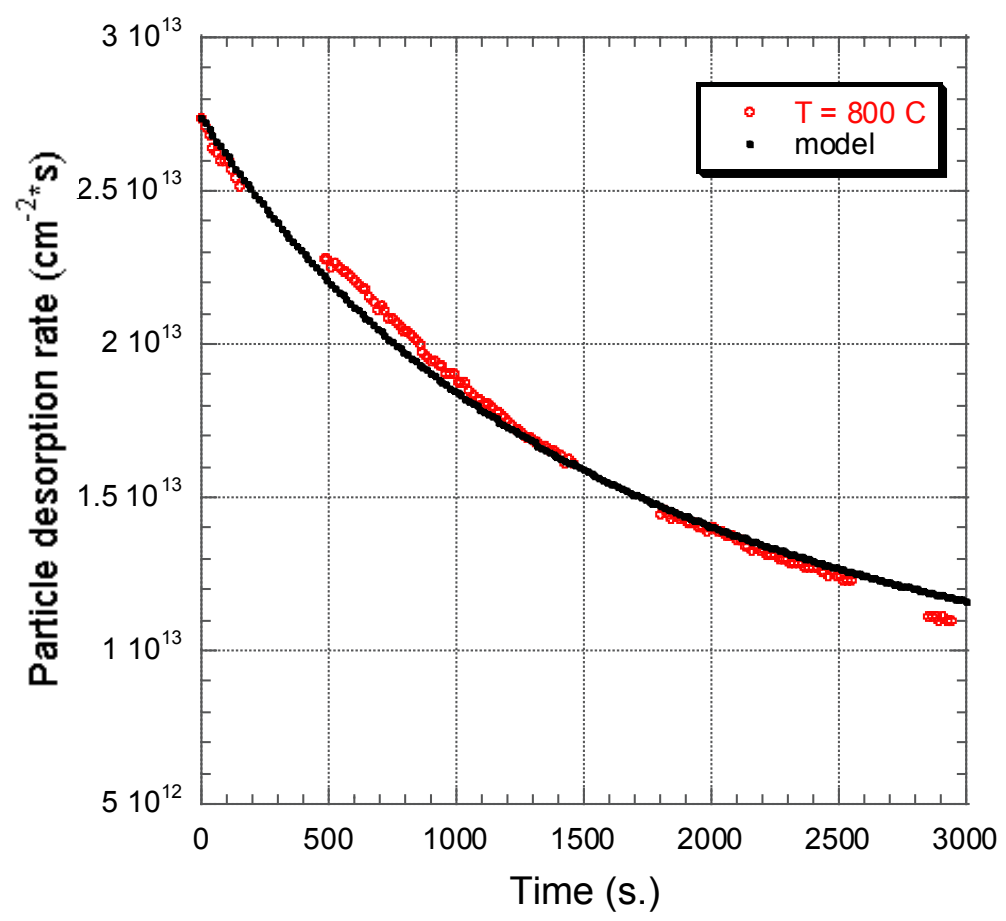

Figure 5.22. Fitting of the Cs neutral desorption data at a temperature of $800{ }^{\circ} \mathrm{C}$. Diffusion constant: $3.7 \cdot 10^{-6} \mathrm{~cm}^{2} / \mathrm{s}$ 


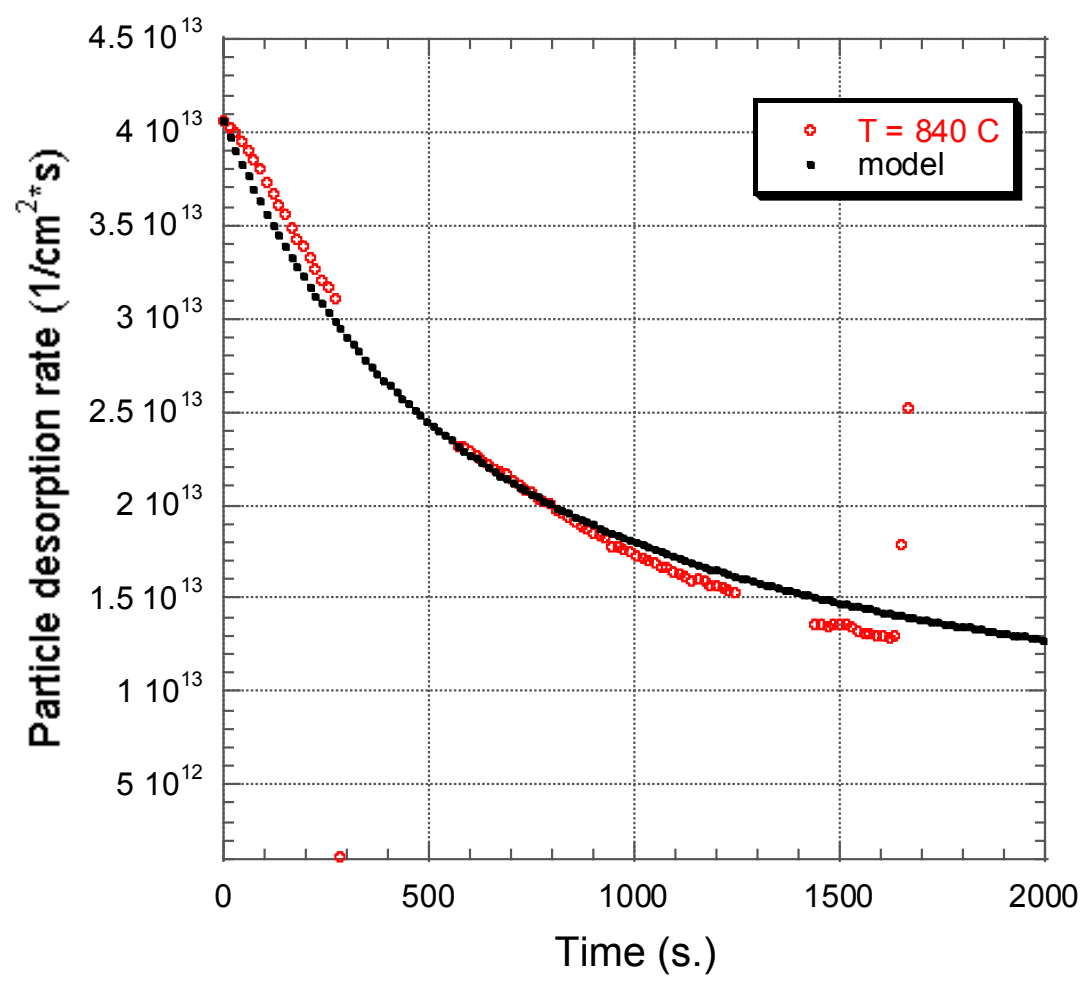

Figure 5.23. Fitting of the Cs neutral desorption data at a temperature of $840{ }^{\circ} \mathrm{C}$. Diffusion constant: $1.010^{-5} \mathrm{~cm}^{2} / \mathrm{s}$.

As the evaporation of neutral particles becomes smaller and smaller, eventually the experiment ran into the problem of actually detecting the digital noise of the high impedance voltmeter used. Figures 5.17 through 5.19 suffer from this digital noise problem. The time intervals during which the measurements were made also included periods when the background signal was been checked, hence the data sets are not continuous. The dispersion of the data points makes the uncertainty of the curve parameters greater, but the estimated values of the diffusion constants still provide a good idea of the diffusion characteristics at the evaluated conditions. These graphs are presented also to give an idea of the type of agreement (good or not so good) obtained. 


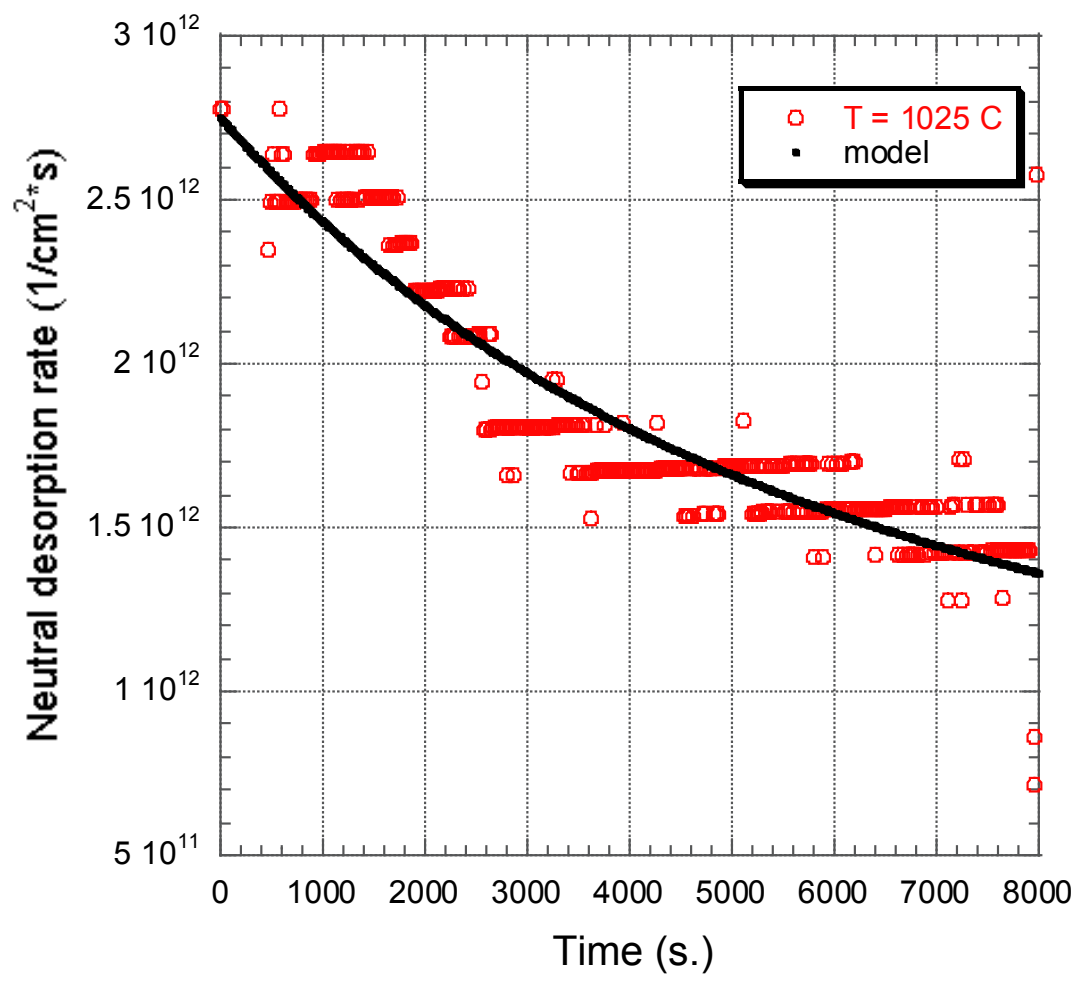

Figure 5.24. Fitting of the Cs neutral desorption data at a temperature of $1,025^{\circ} \mathrm{C}$. Diffusion constant: $1 \cdot 10^{-6} \mathrm{~cm}^{2} / \mathrm{s}$

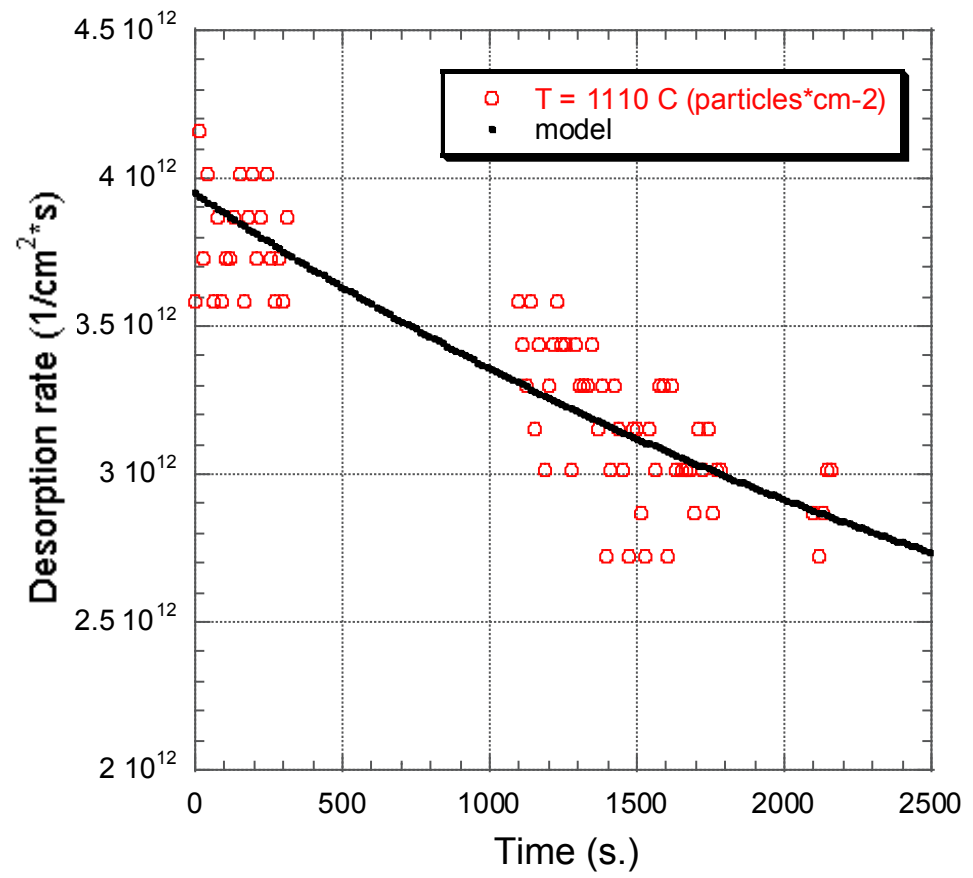

Figure 5.25. Fitting of the Cs neutral desorption data at a temperature of $1,110^{\circ} \mathrm{C}$. Diffusion constant: $7.5 \cdot 10^{-5} \mathrm{~cm}^{2} / \mathrm{s}$ 


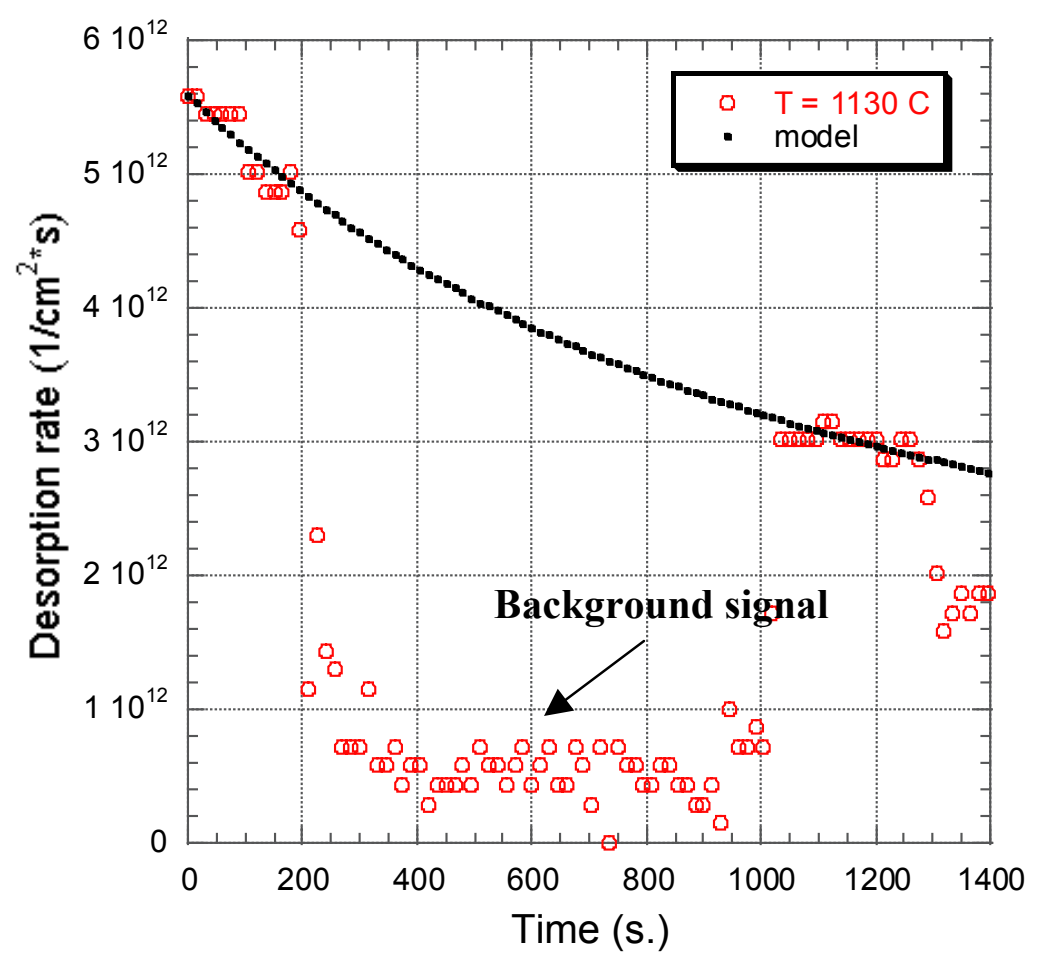

Figure 5.26. Fitting of the Cs neutral desorption data at a temperature of $1,130^{\circ} \mathrm{C}$. Diffusion constant: $5.7 \cdot 10^{-6} \mathrm{~cm}^{2} / \mathrm{s}$

Table 5.4 shows a summary of the different diffusion rates measured under the experimental conditions tested that are of relevance for HIF sources. Note how the diffusion rate is higher at the lower temperatures of the heating cycle while the emitter has a large amount of alkali atoms and the flow through the porous substrate is primarily in the form of gaseous flow.

Figures 5.27 and 5.28 show the $\mathrm{J}-\mathrm{V}$ characteristics of the cesium and potassium sources respectively, at the different temperatures of operation achieved during the neutral particle and diffusion rate measurements. These curves were used to calculate the ratios of maximum available current density to neutral particle desorption shown in Figure 4.60. 


\begin{tabular}{ccc}
\hline & \multicolumn{2}{c}{ Diffusion Coefficient $\left(\mathbf{c m}^{2} / \mathbf{s}\right)$} \\
\hline Temperature $\left({ }^{\circ} \mathbf{C}\right)$ & Cesium & Potassium \\
680 & $5.9 \cdot 10^{-06}$ & \\
765 & $1.2 \cdot 10^{-05}$ & \\
800 & $3.7 \cdot 10^{-06}$ & $4.0 \cdot 10^{-06}$ \\
815 & & $5.0 \cdot 10^{-06}$ \\
840 & $1.0 \cdot 10^{-05}$ & \\
885 & $6.0 \cdot 10^{-06}$ & \\
955 & $4.0 \cdot 10^{-06}$ & $4.0 \cdot 10^{-06}$ \\
955 & $1.1 \cdot 10^{-06}$ & $2.0 \cdot 10^{-06}$ \\
955 & $6.5 \cdot 10^{-07}$ & $1.4 \cdot 10^{-06}$ \\
955 & $5.0 \cdot 10^{-07}$ & $3.0 \cdot 10^{-07}$ \\
1,025 & $1.0 \cdot 10^{-06}$ & \\
1,030 & & $1.6 \cdot 10^{-06}$ \\
1,055 & $1.2 \cdot 10^{-06}$ & \\
1,075 & $8.0 \cdot 10^{-07}$ & $1.8 \cdot 10^{-06}$ \\
1,095 & & $1.5 \cdot 10^{-06}$ \\
1,110 & $1.35 \cdot 10^{-06}$ & \\
1,130 & $5.7 \cdot 10^{-06}$ & \\
1,150 & & $1.9 \cdot 10^{-06}$ \\
1,185 & & $3.5 \cdot 10^{-06}$ \\
\hline
\end{tabular}

Table 5.4. Estimated diffusion constants of the alkali species through a nominal $12 \mu \mathrm{m}$ grain size porous tungsten substrate. Calculated by fitting the measured neutral desorption curves with equation (5.16). Several values for $\mathrm{D}$ are listed for the temperature of $955^{\circ} \mathrm{C}$ as it is at this temperature that the source is left by itself to eliminate the surplus of alkali atoms and during a period that lasts $\sim 17$ hours. Lower diffusion constants are estimated at longer times. In general, the uncertainty of the values can be estimated to \pm 5 in the last decimal place to have curve fits of the type of agreement shown in Figures 5.20 through 5.26. 


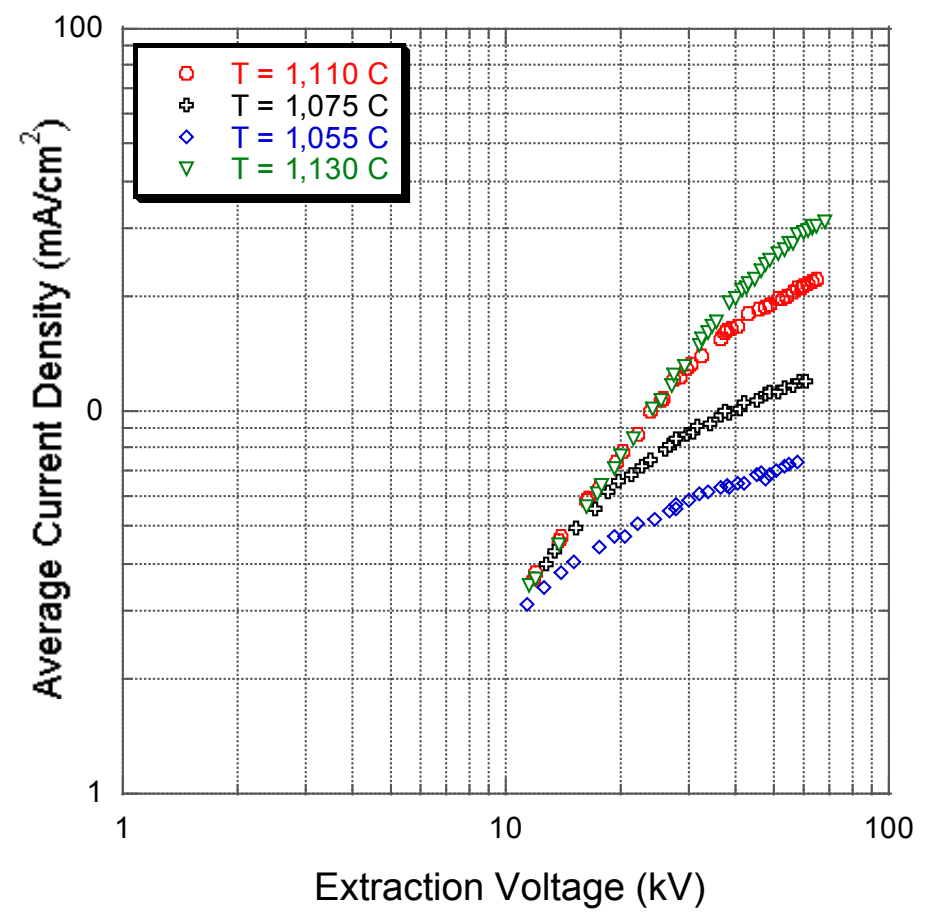

Figure 5.27 $\mathrm{J}-\mathrm{V}$ characteristics of the cesium on tungsten ion source used for testing of the neutral evaporation rates and diffusion constants.

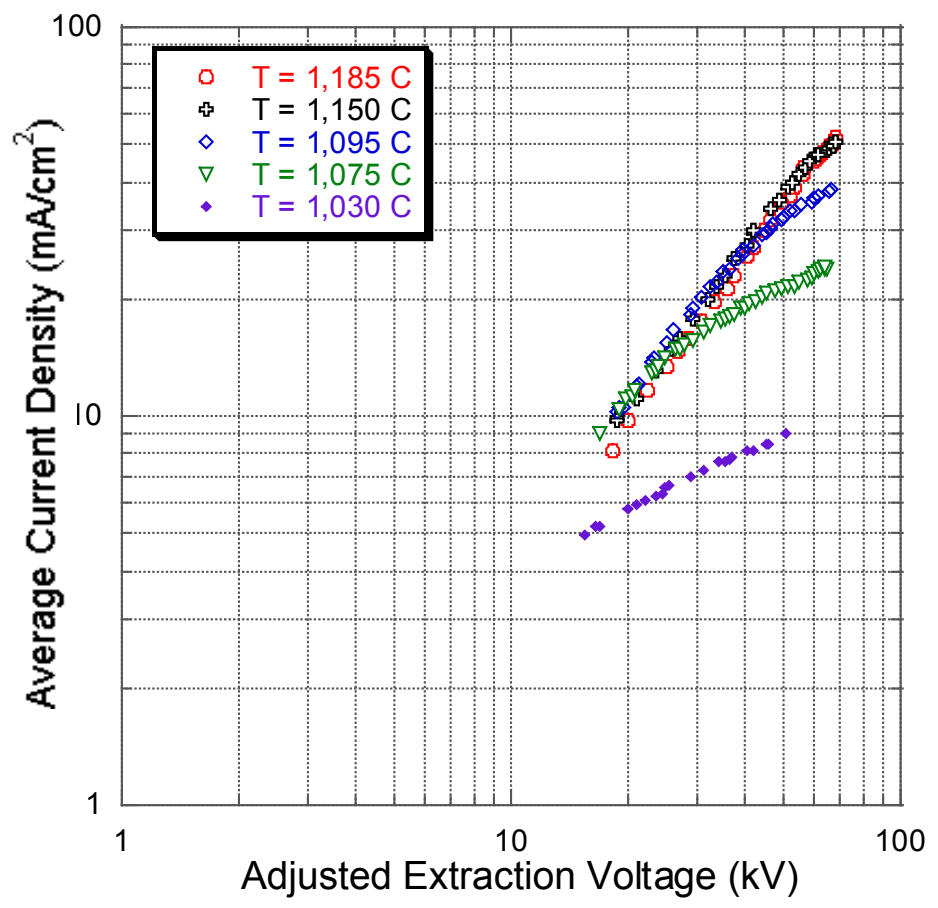

Figure $5.28 \mathrm{~J}-\mathrm{V}$ characteristics of the potassium on tungsten ion source used for testing of the neutral evaporation rates and diffusion constants. 


\subsubsection{On the surface replenishment rate.}

The studies on neutral particle desorption rates show values for these rates that are completely consistent with the observed lifetimes for contact ionization sources. For instance, let us consider the case of a cesium source operating at $1,150{ }^{\circ} \mathrm{C}$, taking a diffusion length of $\mathrm{L}=0.635 \mathrm{~cm}$ (the length of the cylindrical emitter), the associated diffusion time scale would be $\sim \mathrm{L}^{2} / \mathrm{D}=(0.635 \mathrm{~cm})^{2} /\left(5.7 \cdot 10^{-6} \mathrm{~cm}^{2} / \mathrm{s}\right) \cong 19 \mathrm{hrs}$. This is consistent with the observed source lifetimes. This suggests that indeed, for the duty factors used in these experiments, which are extremely low ( $\sim 2 \mu \mathrm{s}$, every $20 \mathrm{~s}$, or $\left.\sim 1 \cdot 10^{-7}\right)$ the main loss mechanism for alkali atoms is indeed the loss as neutral particles.

Data has been shown as well (see section 5.3) regarding the influence of the extracted current on the surface coverage during the current pulse. This data was shown in the context of a depleting surface, where the alkali concentration of the surface is low enough to be affected by the extracted current. Nevertheless, this condition of surface depletion during the pulse can also be achieved with sufficiently large extracted current densities, or sufficiently long high voltage pulses. Since the experimental means available at our facility could not deliver high enough extraction voltages or sufficiently long high voltage pulses, in the following pages the considerations that will be presented are based on theoretical considerations. Nevertheless, these considerations are well grounded on the observations made and presented throughout this discussion.

From the discussion in section 5.3 regarding the current decay during the pulse due to surface depletion, there is an obvious fact that can be appreciated by looking at the different photographs. Even after a current pulse shows signs of surface depletion, if the source is given a sufficient amount of time (in the time scale of seconds), the next pulse 
will be exactly alike, demonstrating that the diffusion of the alkali atoms towards the surface is able to provide the necessary amount of alkali particles for the next current pulse. The surface coverage conditions are recovered by the diffusion mechanism in a certain time scale. This observation immediately leads to the question of what is this time scale, and at what duty factor or repetition rate will an ion source operating in this mode run into a "diffusion limited mode" so that the current demands are greater than the natural, diffusion dominated, replenishment rate of the surface.

The simplest and most direct approach to this problem must consider what is the longitudinal depth of the emitter surface that is actually affected by the extracted current, in order to estimate what is the time scale relevant for diffusion processes at such dimensions. In other words, since up to this moment the modeling has been carried out by considering the particle concentration per unit length (in a unit transverse area of the emitter), we want to know what is the "surface thickness" that is affected (depleted) by the ion pulse. It seems reasonable to assume a "surface thickness" of the same magnitude as the size of the tungsten grains that compose the material. More precisely, a value of half of the nominal grain size should be used, given that such would be the distance between the outermost and innermost surfaces of the tungsten grains on the surface. The length chosen is then $\sim 6 \mu \mathrm{m}$. This is the longitudinal distance along the emitter that would be affected by a current pulse. With this scale, the associated diffusion time (again taking the case at $\left.\mathrm{T}=1,150{ }^{\circ} \mathrm{C}\right)$ would be $\tau_{\mathrm{d}} \sim \mathrm{L}^{2} / \mathrm{D}=\left(6 \cdot 10^{-4} \mathrm{~cm}\right)^{2} / 3.5 \cdot 10^{-6} \mathrm{~cm}^{2} / \mathrm{s}=63.2$ ms. If we further consider that a required time of approximately $5 \tau_{\mathrm{d}}$ for a full replenishment of the surface coverage by diffusion mechanisms after a current pulse, then this would require a total time of $316 \mathrm{~ms}$. With such time requirements, an ion source 
operating in this mode of diffusion replenishment would not be able to operate at a rate higher than $3 \mathrm{~Hz}$. With this logic, high repetition rates would be aided by increasing the diffusion rate. A decrease in the tungsten grain size would not necessarily imply an increase in the repetition rate capability of the source, since the diffusion constant (that goes as described in equation (5.26)) should also decrease by the same factor, thus in principle cancelling out any potential advantage of a decrease in the grain size.

It may be possible though to decouple the dependence of the maximum repetition rate available on the grain sizes in the bulk and at the surface of the material. The emitter can be made with a bulk made of sintered $12 \mu \mathrm{m}$ size grains while sintering smaller grains at the surface layer. In this way, the bulk diffusion properties would be maintained, while decreasing the replenishment time of the outermost layer due to the presence of a smaller diffusion distance. Figure 5.22 shows a general estimate of the maximum achievable repetition rates with such an arrangement. For visualization purposes, three different grain sizes at the surface of the emitter are considered. The curves are calculated by defining the maximum repetition rate (MRR) as:

$$
M R R=\frac{1}{5 \tau_{d}}=\frac{D_{b u l k}}{5 l^{2}}
$$

where "l" is the surface diffusion length, or the length that the ions must replenish after a current pulse. The value of "l" is taken to be half of the nominal size of the metallic grains that form the surface of the emitter. The reasoning behind equation 5.28 is that once the surface is depleted, it will take a time requirement of 5 times the diffusion time constant to recover the original state of surface coverage. At higher repetition rates, different quasi-steady states would be achieved. These states would presumably yield 
lower current densities or pulse drooping with respect to the condition in which a full replenishment is allowed.

Even as a rough estimate, this addresses a real potential limitation for high current density ion sources operating in this diffusion-limited mode with low alkali atom content, like the ones commonly used in HIF experiments. The results presented on Figure 5.29 are at odds with usual HIF driver requirements of operation in the $10 \mathrm{~Hz}$ regime. For this reason some discussion on the possible sources of error on this analysis is warranted.

Two main potential sources of error have been identified. On one hand, one of the basic assumptions that was made was that the phenomenon of diffusion with subsequent loss of particles at one surface is completely diffusion dominated. The values of the $\zeta_{\mathrm{n}}$ parameters in equation 5.16 were chosen for the most extreme case of a diffusion limited process. In the language of the heat transfer problem after which this problem has been modeled, a case was chosen in which the convective heat loss rate was infinitely larger than the conductive propagation of heat. Hence the choice of the Biot number, $\mathrm{Bi} \rightarrow \infty$. This assumption may be challenged, if the phenomenon to be modeled is not entirely diffusion dominated (which given the expected desorption times a the surface, seems very unlikely). In this case, a different set of parameters for equation 5.25 should have been chosen. 


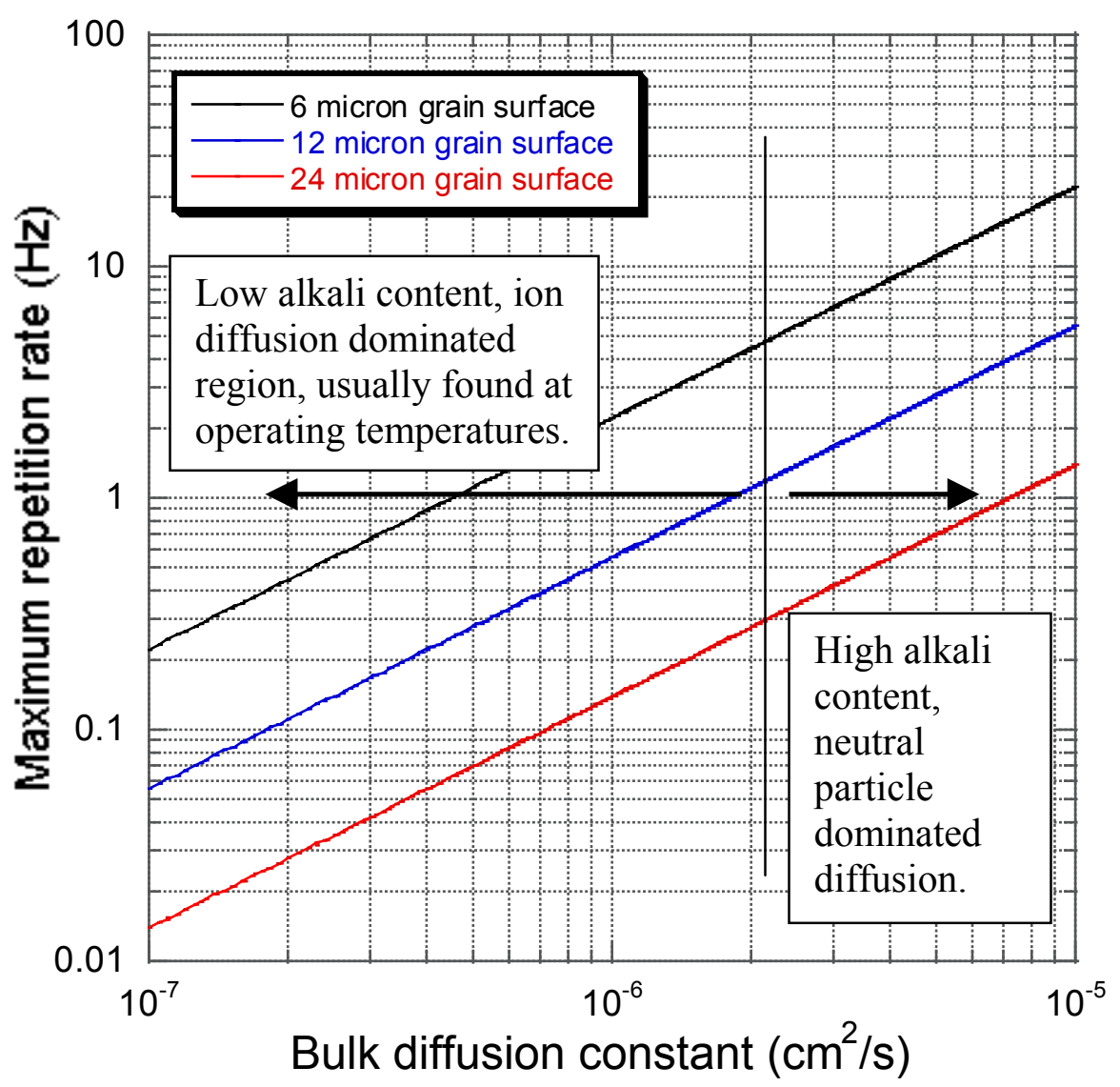

Figure 5.29. Dependence of the maximum repetition rate available in a contact ionization source as a function of the bulk diffusion constant, as measured in a $12 \mu \mathrm{m}$ nominal grain size emitter. The curves are parametrized by the grain size at the surface of the emitter in case that different grain sizes can be used at the surface and in the bulk material. The diffusion length is then taken as half of the nominal grain size at the surface.

Since the set of values for the $\zeta_{\mathrm{n}}$ parameters used in equation 5.25 decrease whenever the diffusion is a less dominant factor, then the analyses made here are a conservative case in which the values of the estimated diffusion coefficients may have been underestimated. For example, a choice of the Biot number of 10, which still models a higher rate of desorption than diffusion, will yield values higher than the originally estimated by approximately $67 \%$. Figure 5.29 has been re-calculated and is shown in Figure 5.30. 


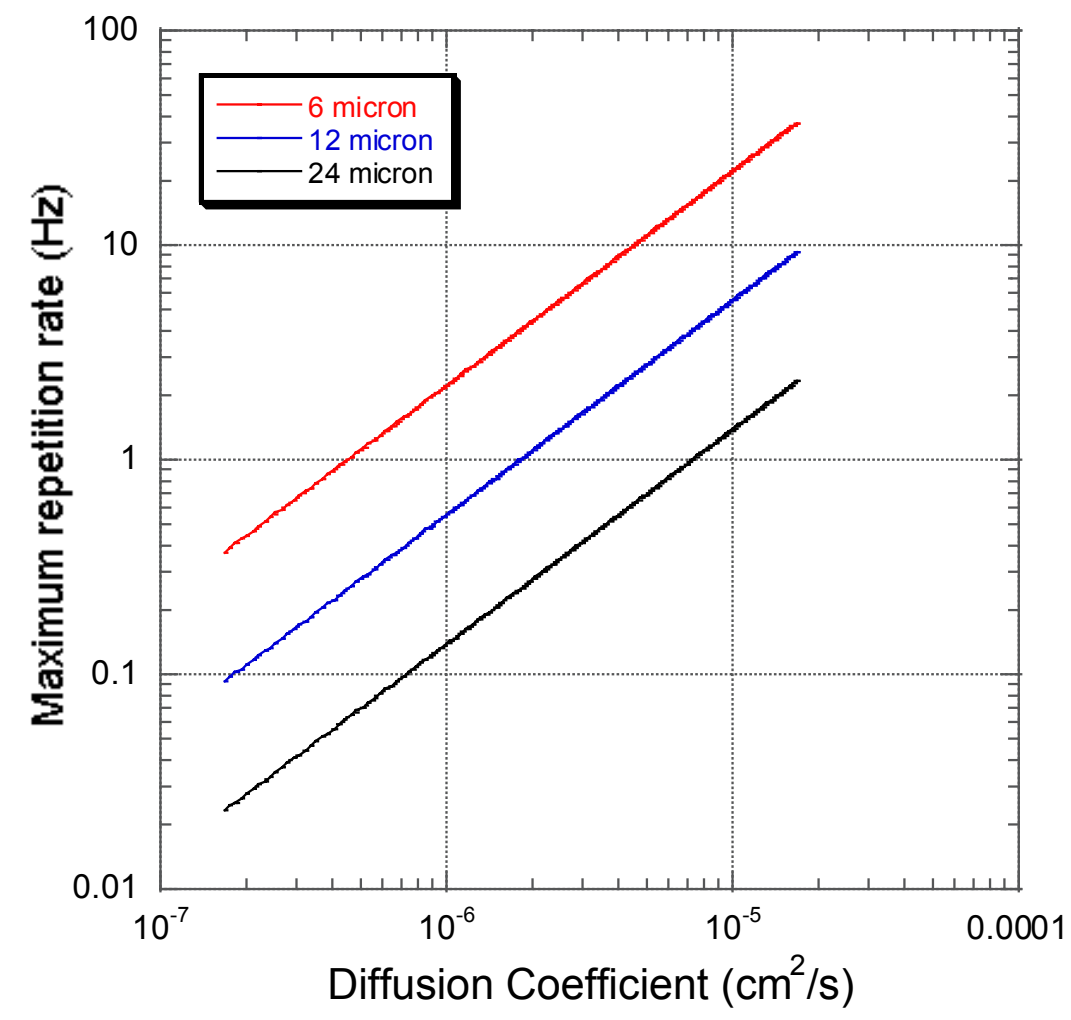

Figure 5.30. Maximum repetition rate estimated with the same methodology as in Figure 5.29. The ratio of the diffusion rate versus the desorption rate was given a higher value, thus making the diffusion constant estimates less conservative. Diffusion constant values differ by a factor of $\sim 1.67$ with respect to the previous figure.

While the desired HIF operation regime $(\sim 10 \mathrm{~Hz})$ may be attained by appropriate selection of the surface diffusion length, this regime is only attained with fast diffusion constants, only attained with a high volume concentration of alkali atoms.

\subsubsection{On the differences of operation between a propulsion ion thruster and the studied contact ionization sources.}

The previous results seem to be at clear odds with previous experiences in contact ionization sources, namely, the ones used in space propulsion applications, in which 
current is extracted on a DC basis. This apparent conflict begs for an explanation. As it will be noted, the ion sources used in such application in reality operate in a very different mode than the sources used in these experiments. The results obtained are thus consistent, and further, necessary to understand the behavior of the ion sources used in HIF experiments up to date.

The operation of a contact ionization source operating in the mode common to HIF experiments was described above, it can be further summarized as follows:

1. An initial stage of high alkali atom content occuring just after the chemical compounds used to dope the source are broken down. The main mode of diffusion of the alkali species is a neutral particle diffusion through the porous substrate through repeated desorption and adsorption cycles. Despite the fact that at low temperatures high ionization is favored, ionization does not occur given the effects of the large alkali concentration on the tungsten surface's work function. During this stage, the diffusion is fast.

2. As the source temperature is increased, the diffusion rates increase for the neutral atoms phase. However, as the total alkali content decreases, the fraction of alkali atoms that becomes ionized starts to raise. Ions have different migration properties due to the magnitude of the force (image force) that attracts them to the metallic surface. The main migration mechanism for ions is a surface diffusion, which is slower than the diffusion of neutral alkali particles.

3. When the alkali content in the source is very low, most of the particle population exists in the ion state. The migration is thus a slow diffusion towards the surface 
where the particles are lost to neutral desorption and ion emission during current extraction. At this point the dynamics of the surface concentration are changing slowly, and it is in this mode that the source usually operates. See Figure 4.16 as an example of the ion output of a Cs source as the surface conditions slowly change. At this point, presumably there exists a condition of quasi-equilibrium, in which there is almost a balance between a very low rate of surface replenishment by diffusion, and a low rate of neutral desorption. Since the desorption time of the neutral particles is not zero, at any given moment there is a population of ions and atoms at the emitting surface. During a short current pulse, the particles at the surface are quickly desorbed as ions. The ratio of ions to neutrals given by the Saha equation remains valid even at very short time scales, since the electronic transitions that may ionize an atom or neutralize an ion occur faster than any other of the relevant processes. After the source has been depleted during a pulse, the previous surface condition is recovered by the diffusion replenishment.

4. The measured values of the effective diffusion coefficients suggest that the main migration mechanism is a surface diffusion of the ion species. When the alkali concentration in the emitter is low, the Saha - Langmuir equation determines a high ratio of the amount of ions to the amount of neutrals, hence the ion effects should dominate. The migration of neutrals through repeated desorption adsorption - desorption cycles is highly determined by the porous geometry. This is evident when contrasting the self-diffusion coefficient of Cs atoms at the typical operating pressures and temperatures. The value of this diffusion coefficient (as calculated from Reid, 1988) would be approximately $1.5 \cdot 10^{4} \mathrm{~m}^{2} / \mathrm{s}$, 
many orders of magnitude higher than what has been measured for the effective diffusion coefficients.

On the other hand, the operation of an ion thruster can be described as follows:

1. Given the demand on current, not in terms of current density but in terms of continuous extraction, the emitter requires a very fast and large replenishment rate. This can only be achieved by using a mode of operation in which the dominant diffusion mechanism is a gaseous flow from the bulk of the material (Brewer, p. 103).

2. High alkali atom content at high operating temperatures demands for a mechanism of continuous back feeding of alkali atoms, driven through the porous emitter by a pressure gradient. Otherwise, the fast diffusion would make it impossible to have the sufficient amount of alkali particles for the continuous operation. This pressure gradient flow of neutral atoms is different in nature of the concentration gradient driven, surface-diffusion ion flow in HIF experimental sources.

3. The predominantly gaseous flow through the porous emitter implies the exhaust of a strong neutral particle efflux from the pores at the surface of the emitter. Depending on the operating conditions, the ion thrusters emitted as much as $15 \%$ of neutral particles at the front surface, creating partial pressures on the front of the emitter of the order of $10^{-5}$ torr of an alkali background gas. Clearly such conditions are not observed in HIF experimental sources. 
4. The surface conditions appropriate for ion emission in the thrusters are achieved by the lower alkali coverage in the regions away from the pores, where the ion existence is thus favored. For this reason the number of pores per unit area and the size of the pores are more critical parameters in the ion thruster mode of operation. A large amount of pores per unit area is desirable for beam uniformity purposes. A minimization of the pore size is desirable because this minimizes the area that acts primarily as a "neutral particle exhaust". In addition, the relative amount of area surrounding the pore, where the ion desorption occurs is maximized. In the case of HIF experimental sources the ratio of ions to neutrals is higher due to the low alkali concentration. For this reason the number of pores per unit area is less critical than in the case of the thrusters. It is nevertheless desirable to reduce the surface rugosity (given by the grain size at the surface) because of its negative impact on the beam emittance. The grain size is linked to the overall porosity and number of pores per unit area.

\subsubsection{Theoretical remarks on the diffusion in porous media.}

As it has been shown, the topic of particle flow by diffusion in porous media is of particular relevance for HIF ion source applications. This section presents a brief summary of the relevant topics in particle diffusion. The goal of this section is to guide the reader towards the relevant issues and topics that must be studied if a more detailed analysis of this process is undertaken. The issues described in the following paragraphs will be essential if the diffusion process is to be modeled in detail, which seems the next logical step in the studies of these ion sources. Most of the information on this section 
has been obtained from the text Diffusion in Gases and Porous Media (Cunningham, 1980). This text includes a variety of relevant references for the interested reader.

The types of diffusive flux in porous materials are classified depending on the type of interaction that the diffusing atoms have with the system walls. These walls are the internal surfaces in the porous material. The porous material is though of as consisting of a structure of "particles" (in our experiment these correspond to the tungsten grains that make up the porous material). With this image in mind there are the following characteristic lengths: a) the mean free path between two atomic collisions $(\lambda)$, b) the average distance between two neighboring "particles" or grains $\left(\lambda_{\mathrm{p}}\right)$ and c) the "particle" radius $\left(\mathrm{r}_{\mathrm{p}}\right)$. The atomic or molecular size is assumed negligible in comparison with these lengths.

As said above, the classification of the different diffusion regimes depends on the relative magnitude of these characteristic lengths. For example, whenever $\lambda<<\lambda_{p}$ and $r_{p}$ $>>\lambda$, there is a total interaction between the atoms that collide with the walls and the other flowing molecules before another interaction with the wall occurs. This condition is known as viscous flux. If the condition $\lambda>>\lambda_{\mathrm{p}}$ occurs, the probability of a moleculemolecule collision is negligible compared to that of a molecule-wall collision. In terms of the Knudsen number, Kn defined as

$$
K n \equiv \frac{\lambda}{\lambda_{p}}
$$

the condition can be written as $\mathrm{Kn} \gg>1$. Such condition defines what is known as Knudsen flux. The Knudsen flux is determined by the thermal velocity of the molecules. This is in contrast with the viscous flux where the molecules, despite chaotically colliding 
among themselves, are being transported in a given direction at a given overall velocity. A calculation of $\lambda$ at the typical operating pressures and temperatures of the HIF emitters indicates that the diffusion occurs in the Knudsen regime. The following table shows the general classification of the different diffusion regimes.

\begin{tabular}{ll}
\hline \multicolumn{1}{c}{ Condition } & \multicolumn{1}{c}{ Regime } \\
\hline$\lambda<<\lambda_{\mathrm{p}} ; \mathrm{r}_{\mathrm{p}}>>\lambda$ & Viscous \\
$\lambda \gg>\lambda_{\mathrm{p}} ; \mathrm{r}_{\mathrm{p}}>>\lambda$ & Knudsen \\
$\lambda \cong \lambda_{\mathrm{p}} ; \mathrm{r}_{\mathrm{p}}>>\lambda$ & Slip \\
$\lambda \gg>\lambda_{\mathrm{p}} ; \mathrm{r}_{\mathrm{p}}<<\lambda_{\mathrm{p}}$ & Pseudo-Knudsen \\
$\lambda<<\lambda_{\mathrm{p}} ; \mathrm{r}_{\mathrm{p}}>>0$ & Molecular \\
$\lambda<<\lambda_{\mathrm{p}} ; \mathrm{r}_{\mathrm{p}} \rightarrow 0$ & Inviscid \\
Molecular size $\cong \lambda_{\mathrm{p}}$ & Configurational \\
Different molecular masses; $\mathrm{r}_{\mathrm{p}}>0$ & Nonequimolar
\end{tabular}

Table 5.5. Classification of diffusive fluxes based on the characteristic scale lengths.

When the pore diameter is large enough for molecular diffusion to prevail, the diffusive flux will be independent of the pore diameter. As the pore diameter or the pressure decreases, the resistance due to atom-wall collisions appears and the flux tends to decrease. Since in the Knudsen regime the flux is proportional to the length of the atomic flights, the Knudsen flux will be proportional to the pore diameter. If the pore 
diameter is decreased to the point at which the molecular/atomic size is no longer negligible compared with the pore diameter, another resistance appears and the Knudsen diffusion gives way to configurational diffusion. The above is an example of how the geometry and conditions of the media determines the type of flow. The relevance of this classification is that the flow regime determines whether the flow will be segregational (different species flowing at different speeds) or if there are losses (of momentum or pressure) in the flow. The reader is referred to the references mentioned above for further details.

The measurements described in the previous section are measurements of "effective" diffusion coefficients. The measurements are specific to the particular pore geometry and porosity found in the emitters tested. The reason for this can be appreciated when thinking about the diffusion mechanism that occurs in the Knudsen regime. On one hand there is a population of neutral atoms that migrates through successive adsorption - desorption - adsorption cycles, on the other, an ion population that migrates through surface diffusion along the walls of the porous structure. In both cases, it can be intuitively seen how it is the geometry of the porous structure that will determine the diffusion rate. There is, however, no simple relation between pore size distribution and permeability (Wiggs, 1958). Permeability is defined as the rate of flow under unit pressure difference, through a unit cube of solid. It is defined for a particular fluid and under the particular experimental conditions at which it is measured. It will suffice to say that many structures exist with the same pore size distribution but very different permeability. 
In addition to the pore size distribution it is important to know the "texture", which is the geometry of the pore assembly. For instance, this property refers to pore characteristics like closed pores, pore length in the direction parallel and perpendicular to the direction of flow, and pores with only one entrance.

Another relevant property used to characterize a porous media is the "tortuosity". This property is defined as the cosine squared of the mean angle between the flow streamlines and the overall pressure gradient. The name of this property is quite meaningful as it refers to how tortuous a path must a particle follow to actually move along the main direction of propagation.

It is expected that the above discussion shows the kind of subtleties that are relevant when trying to describe the diffusion processes from the theoretical standpoint.

\subsection{Estimates of apparent neutral particle coverage.}

We can use the neutral desorption rate to make estimates of the apparent surface coverage of alkali atoms on the emitter surface. In order to maintain the levels of neutral particle desorption, it is reasonable to suppose that a quasi-steady state condition is achieved by which the arrival rate of particles by diffusion equals the observed neutral desorption rates.

$$
J_{d}=-D \frac{\partial C}{\partial x}=\left.\left.D C C_{1} \exp \left(-\varsigma_{1}^{2} \frac{D t}{L^{2}}\right)\right|_{t=0} \cdot \sin \left(\varsigma_{1} \frac{x}{L}\right) \frac{\varsigma_{1}}{L}\right|_{x=L}=v_{a}
$$

As an example we can use a typical level of particle desorption rate observed at operating temperatures, namely a level $\sim 7 \cdot 10^{11}$ particles $/ \mathrm{cm}^{2} \mathrm{~s}$. Substituting in the previous equation the values of the constants, using the value of the diffusion constant 
corresponding to what was measured at the above mentioned neutral desorption rate, and solving for $\mathrm{C}$, the particle concentration per unit volume we find:

$$
C \cong 1.6 \cdot 10^{16} \mathrm{~cm}^{-3}
$$

at $\mathrm{x}=\mathrm{L}$ (the position of the surface). It is here necessary to approximate the actual particle density per unit area corresponding to this volume density. We may further approximate the value of the desired parameter, $\theta \sigma_{1}$, by

$$
\theta \sigma_{1}=C^{\frac{2}{3}}
$$

which immediately yields

$$
\theta \sigma_{1}=6.4 \cdot 10^{10} \mathrm{~cm}^{-2}
$$

of apparent concentration of neutral atoms on the surface. This number should constitute a lower bound for the concentration of atoms on the surface, as there could be a higher concentration because of at least two reasons:

a. At any given moment, there is a fraction of the total population of alkali atoms on the surface that exists in the ion form. In other words, there is a definite probability of ionization for the alkali atoms. The total fraction of the population that becomes ionized is given by the Saha-Langmuir equation (5.1) at low surface coverages. Whenever there are changing fractions of the ion or atom populations, the short time scales during which the ionization/neutralization may occur, will preserve the ratio of ions to neutrals given by Saha in a practically instantaneous way.

b. At any given time, only a fraction of the neutral particles may be escaping. Although given the short residence time of a neutral particle on the surface, this is not expected to be a dominant factor. 
The value of the surface coverage is thus highly uncertain, since the ratio of ions to neutral particles on the surface will be a very strong function of the effective work function of the surface, which is an unknown parameter. Furthermore, the estimate of the neutral particle coverage is really an estimate of the "apparent" neutral coverage, since the desorbing neutrals may also be created by the ions that become neutralized, in an attempt to preserve the ratio given by the Saha equation. More details on this mechanism are discussed below, in the following section.

It is not appropriate to "adjust" the estimated value of the apparent neutral concentration to estimate the concentration of ions on the surface. This could in principle be done by simply applying the factor of $n_{i} / n_{a}$ given by the Saha equation. Such estimate of the ion coverage would not be realistic, given that the apparent concentration of neutral particles also includes the fraction of the ion population that is continuously being transformed into neutral particles, thus making the "apparent" concentration, larger than the "real" concentration. For this reason, the apparent concentration of neutrals on the surface tends to strongly overestimate the actual neutral particle concentration that would exist if there was a steady state condition on the surface of the emitter. Figures 5.31 and 5.32 show plots with the estimated "apparent" neutral particle coverage as measured at the beginning of the intervals at each temperature. These plots are for information purposes since the time intervals at each temperature were not equal, thus making the identification of trends in the graphs elusive. 


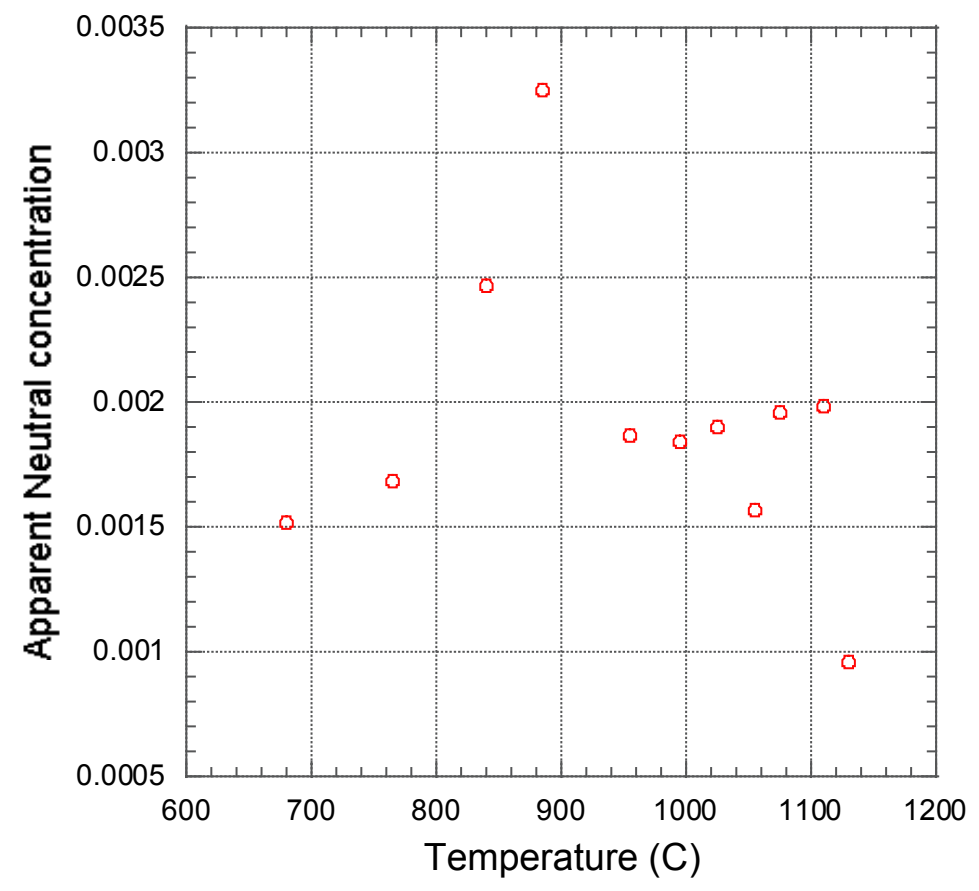

Figure 5.31. Apparent neutral concentration of cesium atoms on the porous tungsten surface (12 $\mu \mathrm{m}$ nominal grain size), as estimated from the neutral particle desorption rate data at the beginning of the time period at each temperature. Time periods were not of the same length.

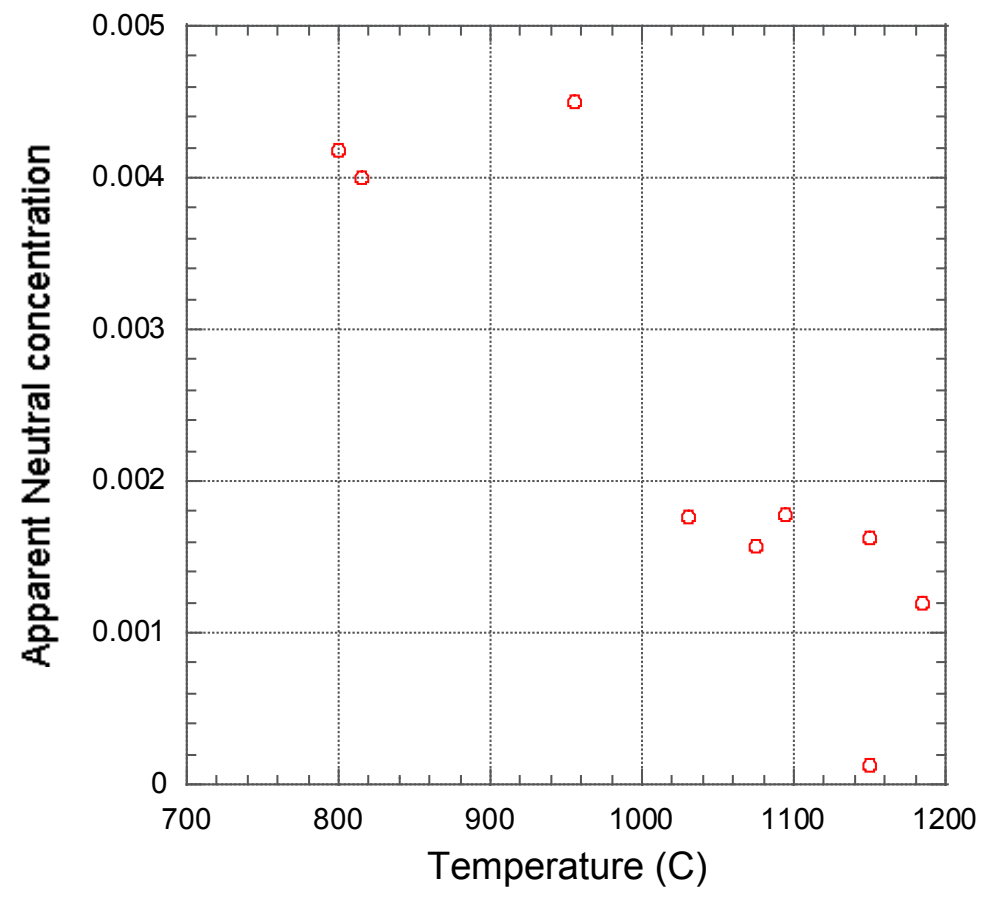

Figure 5.32. Apparent neutral concentration of potassium atoms on the porous tungsten surface (12 $\mu \mathrm{m}$ nominal grain size), as estimated from the neutral particle desorption rate data at the beginning of the time period at each temperature. Time periods were not of the same length. 
It was mentioned above that the apparent concentration of neutral particles may not be readily used to make an estimate of the actual ion coverage. To support this claim it was mentioned how there is a continual transformation of ions to atoms and atoms to ions in such rates that the equilibrium concentration predicted by the Saha equation was maintained. This mechanism also means that once there is a change on either of the ion or atom populations, there will be an imbalance that will be compensated by a net transformation of the other population. One can imagine that the ratio of the rate at which the atoms are transformed into ions, to the rate at which ions are transformed to atoms is what is given by the Saha equation. The population would then naturally drift from any initial ratio of ions to neutrals to the known ratio of species predicted by the Saha equation. This will perhaps be appreciated more clearly with the following exercise.

Let us consider a closed system composed of an arbitrary number of ions, $\mathrm{n}_{\mathrm{i}}$, and an arbitrary number of atoms, $\mathrm{n}_{\mathrm{a}}$. Both populations may live on the surface of a metallic substrate that may affect the probability of an atom becoming ionized or an ion becoming neutralized, as it occurs in the tungsten ionizers under consideration. We view the population of particles on the surface as two sets of discrete species, atoms and ions, with a defined probability of becoming a member of the other set. Let us name the ratio at which atoms are ionized as $\mathrm{R}_{\mathrm{ai}}$ and the ratio at which ions are neutralized as $\mathrm{R}_{\mathrm{ia}}$. The claim is that the ratio of these rates, $\mathrm{R}_{\mathrm{ai}} / \mathrm{R}_{\mathrm{ia}}=1 / 2 \exp [\mathrm{e}(\phi-\mathrm{I}) / \mathrm{kT}]=\mathrm{S}$, is what is determined by the surface, and that with such premise, any population initial ratio $\mathrm{n}_{\mathrm{i} 0} / \mathrm{n}_{\mathrm{a} 0}$ will naturally arrive at the expected equilibrium ratio, $\mathrm{S}$ predicted by the Saha-Langmuir 
equation. To show this, consider the ordinary differential system that describes the rate of change of each of these populations, ions and atoms (equations 5.33, 5.34 and 5.35). Naturally, the only mechanisms for loss or gain in each population are the transformation rates from and to the original states, that is, no replenishment mechanisms or loss mechanisms are being considered, a requirement that does not change the physics of this model.

$$
\begin{gathered}
\frac{d n_{i}}{d t}=R_{a i} n_{a}-R_{i a} n_{i} \\
\frac{d n_{a}}{d t}=-R_{a i} n_{a}+R_{i a} n_{i} \\
R_{a i}=S \cdot R_{i a}=R
\end{gathered}
$$

With the premise of the analysis, equation (5.36), the system of equations may further be written as:

$$
\begin{gathered}
\frac{d n_{i}}{d t}=S R n_{a}-R n_{i} \\
\frac{d n_{a}}{d t}=-S R n_{a}+R n_{i}
\end{gathered}
$$

The solution of the above differential system (equations (5.37) and (5.38)), subject to:

$$
\begin{gathered}
\left.n_{i}(t)\right|_{t=0}=n_{i 0} \\
\left.n_{a}(t)\right|_{t=0}=n_{a 0}
\end{gathered}
$$

is given by:

$$
\begin{aligned}
n_{i}(t) & =n_{i 0} \frac{\left(e^{-R(1+S) t}+S\right)}{1+S}-n_{a 0} \frac{\left(e^{-R(1+S) t}-1\right) S}{1+S} \\
n_{a}(t) & =-n_{i 0} \frac{\left(e^{-R(1+S) t}-1\right)}{1+S}+n_{a 0} \frac{\left(1+S \cdot e^{-R(1+S) t}\right)}{1+S}
\end{aligned}
$$


The equilibrium populations of ions and atoms are given respectively by:

$$
\begin{aligned}
& \lim _{t \rightarrow \infty} n_{i}(t)=\frac{S}{1+S}\left(n_{i 0}+n_{a 0}\right) \\
& \lim _{t \rightarrow \infty} n_{a}(t)=\frac{1}{1+S}\left(n_{i 0}+n_{a 0}\right)
\end{aligned}
$$

from which it follows immediately that the equilibrium ratio of ions to atoms in the system is then:

$$
\lim _{t \rightarrow \infty} \frac{n_{i}(t)}{n_{a}(t)}=S=\frac{1}{2} \exp \left(\frac{e(\phi-I)}{k T}\right)
$$

as expected from the steady state interpretation of the Saha equation. So, starting from an arbitrary ratio of the amounts of ions and neutrals, the requirement on the ratio of the rates of ionization and neutralization will necessarily produce a steady state ion/neutral distribution consistent with the Saha equation prediction. This seems to be a compelling argument to visualize the inter-transformation of the species, ions and atoms, on the surface of an emitter, as a highly dynamical process. For instance, once the effects of desorption of either or both of the species is incorporated, the balance implied in the previous analysis would be disrupted. If there is a continuous desorption of atoms, there would be an imbalance in the equilibrium reactions $n_{i} \Leftrightarrow n_{a}$ such that the ions would be neutralized at a faster net rate than in the equilibrium case, thus becoming an additional source of neutrals. Conversely, during ion extraction, the neutral population would become also a source of ions. As mentioned before, the electronic transitions that ionize or neutralize the particles occur at a very short time scale compared to the extraction pulse times, therefore, their effect during the extraction times would be noticeable. 


\subsection{On the presence of large amounts of ionizable impurities and their effect on the space charge limit.}

The following exercise is of theoretical interest since it is unlikely to have the sufficient amount of ionizable impurities in a source. It may nevertheless give some insight into what happens when there is more than one ionizable species in the emitter.

The standard derivation of the non-relativistic Child-Langmuir limit for charged particle emission of a single species is derived in the one-dimensional case by considering the following equations:

$$
\begin{gathered}
\frac{d^{2} \phi}{d x^{2}}=-\frac{\rho}{\varepsilon_{0}} \\
J=\rho v \\
v=\left(\frac{2 q e \phi}{m}\right)^{1 / 2}
\end{gathered}
$$

This analysis considers negative particles, so as to keep the values of the potential greater than zero, the physical meaning of the results is unaffected. Substituting (5.48) into (5.47), then solving for $\rho$ in the resulting equation and inserting this result in (5.46) yields:

$$
\frac{d^{2} \phi}{d x^{2}}=-\frac{J}{\varepsilon_{0}}\left(\frac{2 q e}{m}\right)^{-1 / 2} \phi^{-1 / 2}
$$

Solution of this equation can be obtained by guessing a solution of the form

$$
\phi(x)=C x^{\beta}
$$

Substituting in (5.40), it is found that

$$
\phi(x)=\left(\frac{9 J}{4 \varepsilon_{0}}\right)^{2 / 3}\left(\frac{2 q e}{m}\right)^{-1 / 3} x^{4 / 3}
$$


Applying the boundary conditions $\phi(0)=0$ and $\phi(\mathrm{d})=\mathrm{V}$, and solving for J one obtains:

$$
J=\frac{4 \varepsilon_{0}}{9}\left(\frac{2 q e}{m}\right)^{1 / 2} \frac{V^{3 / 2}}{d^{2}}
$$

Equation (5.52) is the Child-Langmuir limit for space-charge flow. A similar procedure may be applied to find what is the limiting current value when there is more than one ionizable species at the source. The analysis would again consider only steady state conditions and would therefore not describe possible effects due to a faster ion desorption of the lighter species, but this method may be appropriate to estimate the total ion yield. Here we consider the case of two species with different masses, both with a single charge state, as would be the case of $\mathrm{K}^{+}$and $\mathrm{Cs}^{+}$in a tungsten ionizer. In this case, the space charge in the gap will be composed of the contributions by each of the species. At the same time, the velocities at a fixed position in the gap will be different due to the mass difference. The relevant equations become:

$$
\begin{gathered}
\frac{d^{2} \phi}{d x^{2}}=-\left(\frac{\rho_{1}+\rho_{2}}{\varepsilon_{0}}\right) \\
J=\rho_{1} v_{1}+\rho_{2} v_{2} \\
v_{1}=\left(\frac{2 q_{1} e \phi}{m_{1}}\right)^{1 / 2} \\
v_{2}=\left(\frac{2 q_{2} e \phi}{m_{2}}\right)^{1 / 2}
\end{gathered}
$$

By substituting the expressions of the velocities with the expressions given in (5.55) and (5.56) we arrive at:

$$
J=(2 e \phi)^{1 / 2}\left(\rho_{1} \sqrt{\frac{q_{1}}{m_{1}}}+\rho_{2} \sqrt{\frac{q_{2}}{m_{2}}}\right)
$$


Equations (5.53) and (5.57) form a system of two equations with three unknowns. Since the space charge in the gap is expected to be composed of a determined ratio of the two species present, it is convenient to introduce the parameter $R=\rho_{2} / \rho_{1}$ to solve this system (further considerations will assist in finding out what this ratio is). This would be the ratio of "impurity" ions in the surface to "expected". The new system of equations becomes:

$$
\begin{gathered}
\frac{d^{2} \phi}{d x^{2}}=-\frac{\rho_{1}}{\varepsilon_{0}}(1+R) \\
J=(2 e \phi)^{1 / 2} \rho_{1} \frac{\sqrt{q_{1} m_{2}}+R \sqrt{q_{2} m_{1}}}{R \sqrt{m_{1} m_{2}}}
\end{gathered}
$$

So mathematically the system is reduced to the problem described above for a single species. The resulting ordinary differential equation is:

$$
\frac{d^{2} \phi}{d x^{2}}=-\frac{J}{\varepsilon_{0}}(2 e)^{-1 / 2}(R+1)\left(\frac{\sqrt{q_{1} m_{2}}+R \sqrt{q_{2} m_{1}}}{\sqrt{m_{1} m_{2}}}\right)^{-1} \phi^{-1 / 2}
$$

Solving for the potential we find:

$$
\phi(x)=\left(\frac{9 J}{4 \varepsilon_{0}}\right)^{2 / 3}(2 e)^{-1 / 3}(R+1)^{2 / 3}\left(\frac{\sqrt{q_{1} m_{2}}+R \sqrt{q_{2} m_{1}}}{\sqrt{m_{1} m_{2}}}\right)^{-2 / 3} x^{4 / 3}
$$

and solving finally for the current density after applying the same boundary conditions as above:

$$
J=\frac{4 \varepsilon_{0}}{9}(2 e)^{1 / 2}\left(\frac{\sqrt{q_{1} m_{2}}+R \sqrt{q_{2} m_{1}}}{(R+1) \sqrt{m_{1} m_{2}}}\right) \frac{V^{3 / 2}}{d^{2}}
$$


It may be convenient to further simplify the above expression by introducing the ratio $\kappa=\mathrm{m}_{2} / \mathrm{m}_{1}$, that is, the ratio of the mass of the "impurity" ion compared to the "expected" one. We obtain then:

$$
J=\frac{4 \varepsilon_{0}}{9}\left(\frac{2 e}{m_{1}}\right)^{1 / 2}\left(\frac{\sqrt{q_{1} \mathrm{~K}}+R \sqrt{q_{2}}}{(R+1) \sqrt{\mathrm{K}}}\right) \frac{V^{3 / 2}}{d^{2}}
$$

What is the ratio $R=\rho_{2} / \rho_{1}$ ? It is necessary to cast this parameter in terms of other quantities. Using the relation $\rho_{\mathrm{i}}=\mathrm{J} / \mathrm{v}_{\mathrm{i}}$ and equations (5.55) and (5.56), the ratio $\mathrm{R}$ can be expressed as in (5.64).

$$
R=\frac{J_{2}}{J_{1}}\left(\frac{q_{1} m_{2}}{q_{2} m_{1}}\right)^{\frac{1}{2}}=\frac{J_{2}}{J_{1}}\left(\frac{q_{1}}{q_{2}} \mathrm{~K}\right)^{\frac{1}{2}}
$$

Equation (5.63) can thus be re-written as

$$
J=\frac{4 \varepsilon_{0}}{9}\left(\frac{2 q_{1} e}{m_{1}}\right)^{\frac{1}{2}}\left(\frac{1+\frac{J_{2}}{J_{1}}}{\frac{J_{2}}{J_{1}}\left(\frac{q_{1}}{q_{2}} \mathrm{~K}\right)^{\frac{1}{2}}+1}\right) \frac{V^{\frac{3}{2}}}{d^{2}}
$$

Equation (5.65) is the formal solution to the problem. The original solution shown in equation (5.52) has been modified by the term within the large parenthesis. Equation (5.52) can be recovered by setting $\mathrm{J}_{2}=0$ in (5.65). We are still left with the need to estimate the ratio $J_{2} / J_{1}$. It should be recognized here that this ratio depends on the actual capability of the emitter to deliver ions of either species. In other words, the ratio $J_{2} / J_{1}$ depends on the relative concentration of the species at the emitter surface. With the relation (5.65), once the total current density $(\mathrm{J})$ is measured experimentally, in principle it could be possible to estimate the ratio $\mathrm{J}_{2} / \mathrm{J}_{1}$ by measuring the deviation of $\mathrm{J}$ 
with respect to the value it would have if only one of the species were present. Of course this assumes that only one other species can actually be emitted and also assumes knowledge of the emitted charge states. This may be unrealistic except for alkali species where $\mathrm{q}=1$.

The estimate of the $\mathrm{J}_{2} / \mathrm{J}_{1}$ term may allow for a further estimate of the ratio of the surface concentrations. If the surface concentration of either species is low enough for equation (5.12) to hold, then the ratio of the current densities would be related to the concentrations by

$$
\frac{J_{2}}{J_{1}}=\frac{v_{p 2}}{v_{p 1}}=\frac{\theta_{2} \sigma_{2}}{\theta_{1} \sigma_{1}} \exp \left(\frac{Q_{1}-Q_{2}}{k T}\right)
$$

which again presupposes previous knowledge of the values of the desorption parameters $(\mathrm{Q})$ and the monolayer coverage constant $(\sigma)$ for both species.

\subsection{On the effects of residual atmospheres of alkali gases on the ion source current output.}

Since the demand of current density at the regimes of interest in this work may have a significant impact on the surface coverage over the pulse time, it is important to look at the possible mechanisms that may contribute to the maintaining a particular level of surface coverage. This aspect was a necessary consideration given the background signal observed with the neutral particle detector. Although small, the signal was not zero and one of the possibilities for this signal is that there is an incoming influx of neutral alkali atoms in the residual atmosphere in the vacuum tank.

From kinetic theory, it is known that the rate, $\mu$ (in particles per unit area per unit time) at which particles from a gas strike a surface is given by: 


$$
\mu=p(2 \pi m k T)^{-\frac{1}{2}}
$$

where $\mathrm{p}$ is the gas pressure, $\mathrm{m}$ is the mass of the particles in the gas, $\mathrm{k}$ is Boltzmann's constant and $\mathrm{T}$ is the temperature of the gas. Upon converting the background signal in the detector into an incoming particle density (assuming a 100\% adsorption with subsequent ionization, and a conservative temperature of $300 \mathrm{~K}$ ), the corresponding residual gas pressure of alkali atoms was estimated to be $4 \cdot 10^{-11}$ torr. This is a much lower pressure than the ordinary operating pressure in the experimental facility, of $\sim 5 \cdot 10^{-7}$ torr, thus making plausible the idea that a very tenuous atmosphere of alkali atoms indeed exists in the chamber. If it is assumed that this residual atmosphere is indeed present, then it must be accepted that the source itself is receiving an influx of alkali atoms. At this estimated pressure of $4 \cdot 10^{-11}$ torr, the influx of particles would have a magnitude of $1.1 \cdot 10^{10}$ particles $/ \mathrm{cm}^{2} \mathrm{~s}$. This means that on a $2 \mu$ s extraction pulse, there is a number of $2.2 \cdot 10^{4}$ particles arriving on the surface. Since the number of particles leaving the surface in a $90 \mathrm{~mA} / \mathrm{cm}^{2}$ pulse is $1.125 \cdot 10^{12}$ particles $/ \mathrm{cm}^{2}$, it can be concluded that the effect on the actual surface coverage that this residual atmosphere has is negligible.

In order to provide appreciable levels of replenishment, namely, incoming particle fluxes of the order of $10^{12}$ particles $/ \mathrm{cm}^{2}$, basically all of the residual gas in the vacuum chamber would need to be composed of alkali atoms, that is, a partial pressure of $\sim 4 \cdot 10^{-7}$ torr.

It can be concluded then, that based on these considerations and on the measured background signal detected by the neutral particle detector, partial replenishment of the alkali atom coverage in the emitter due to the residual alkali atmosphere in the tank is not an important factor in these experiments. 


\subsection{Ion Gun Simulations and Sensitivity Analyses.}

Besides the initial ion gun simulation used for the design of the extraction system, further particle tracking simulations were made to better understand the potential effects of the emitter-Pierce electrode gap.

In surface ionization sources of large (multi-cm) diameter, it is possible to identify the influence of edge effects, namely non-uniformities in the extraction or effects caused in the emitter surface - Pierce electrode transition. In the case of small (mmsized) sources, since the ratio of the perimeter of the source to the total area is larger, the influence of these effects was expected to be significantly larger.

The software package used to model the influence of these effects was "TRAK $5.0^{\circledR}$, , written by Stanley Humphries of the University of New Mexico. Trak ${ }^{\circledR}$ is an electrostatic code with particle tracking suitable for simulations of space charge limited emission situations such as the one in hand. Figure 5.32 shows a typical Trak output of the used setup.

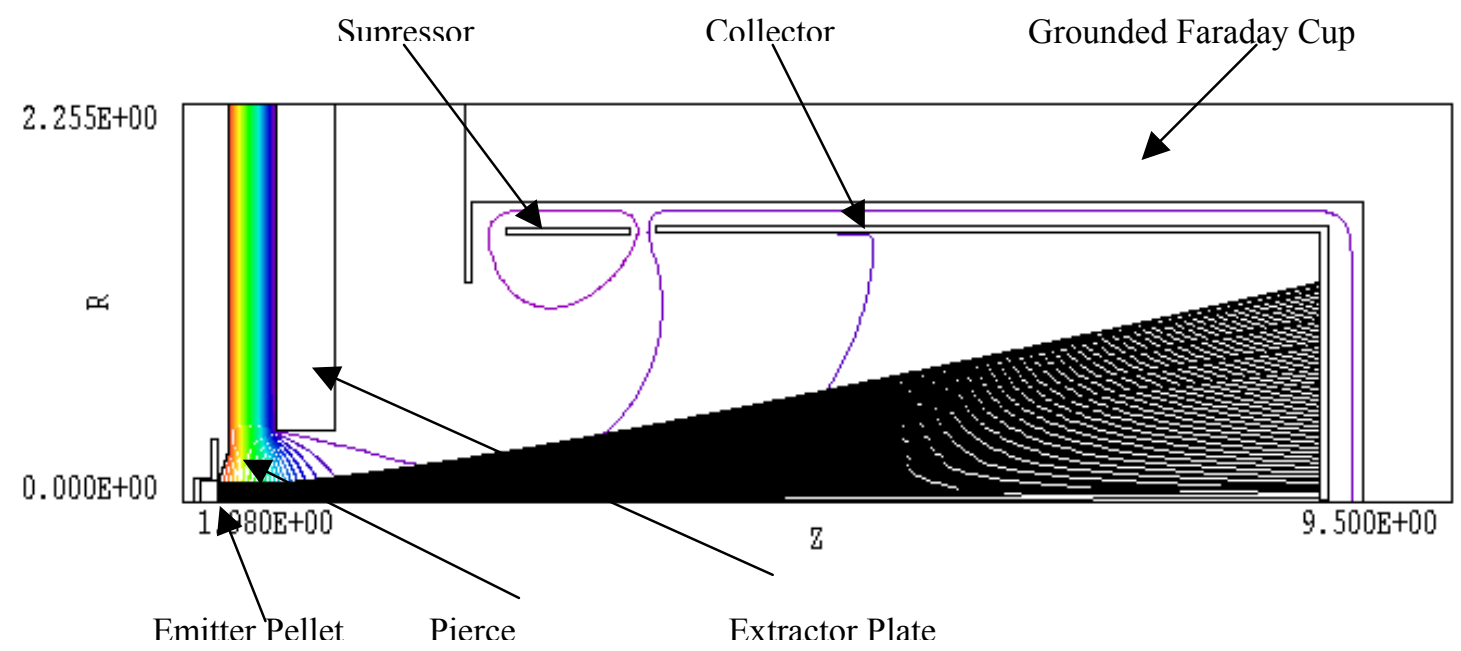

Figure 5.32. Solution region for the modeling of the beam extraction with Trak 5.0. 


\subsubsection{Modeling of the gap geometry.}

Particular care was taken in the modeling of the extraction geometry, with an emphasis on the emitter surface - Pierce electrode gap. The following Figure 5.33 show the main features modeled, as well as the principal effect of a decreasing gap.

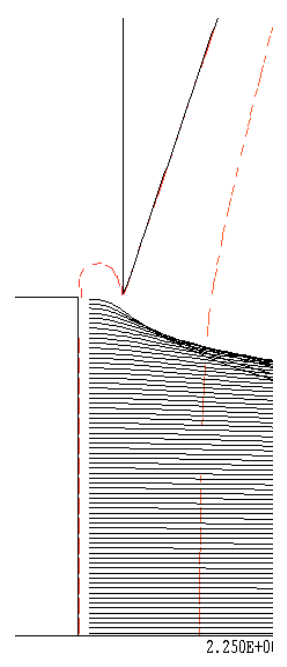

17 mils gap

$\sim 0.43 \mathrm{~mm}$

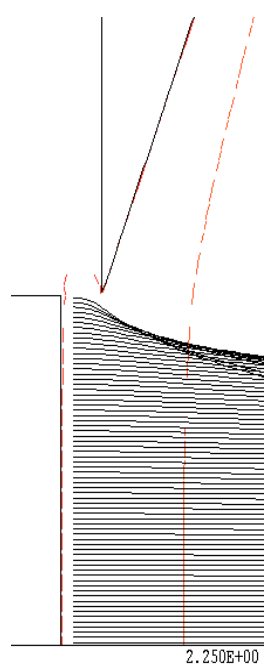

15 mils gap

$\sim 0.38 \mathrm{~mm}$

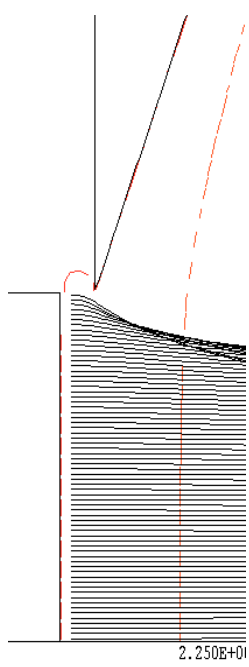

13 mils gap

$\sim 0.33 \mathrm{~mm}$

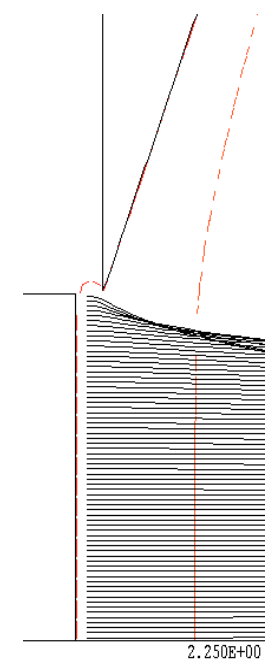

10 mils gap $\sim 0.25 \mathrm{~mm}$

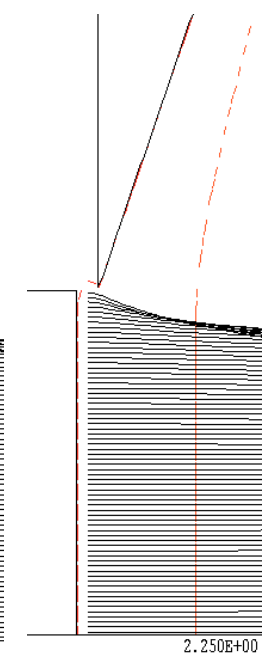

8 mils gap $\sim 0.20 \mathrm{~mm}$

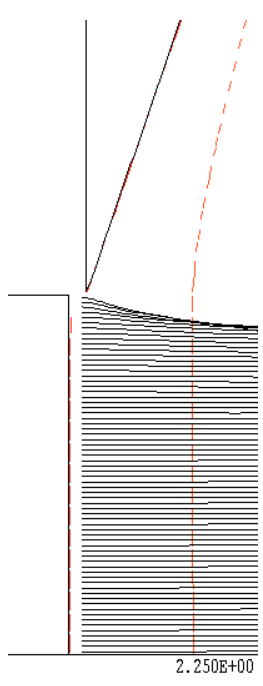

6 mils gap $\sim 0.15 \mathrm{~mm}$

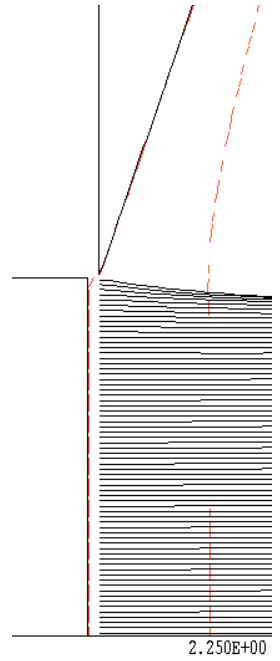

4 mils gap $\sim 0.1 \mathrm{~mm}$

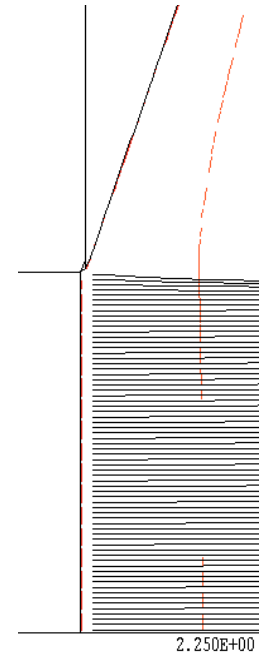

2 mils gap $\sim 0.05 \mathrm{~mm}$

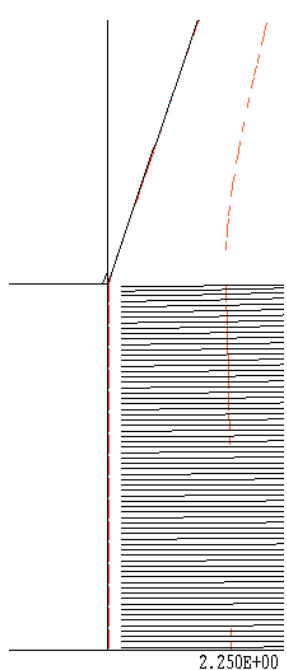

no gap

Figure 5.33. Trak 5.0 modeling of the Pierce - emitter gap influence on the initial extraction optics. 
There are two ways to see in a quantified manner the effects of the gap on the produced beam. On one hand we have a strong effect on the gun perveance. Figure 5.34 shows the different current densities predicted at different voltages with the emitter Pierce gap as a parameter. It can be seen how at an extraction voltage of $30 \mathrm{kV}$, for instance, the emitted current will have any value between 25 and $45 \mathrm{~mA} / \mathrm{cm}^{2}$ depending on the gap. The other way is by showing the effect on the current density profile, as shown below.

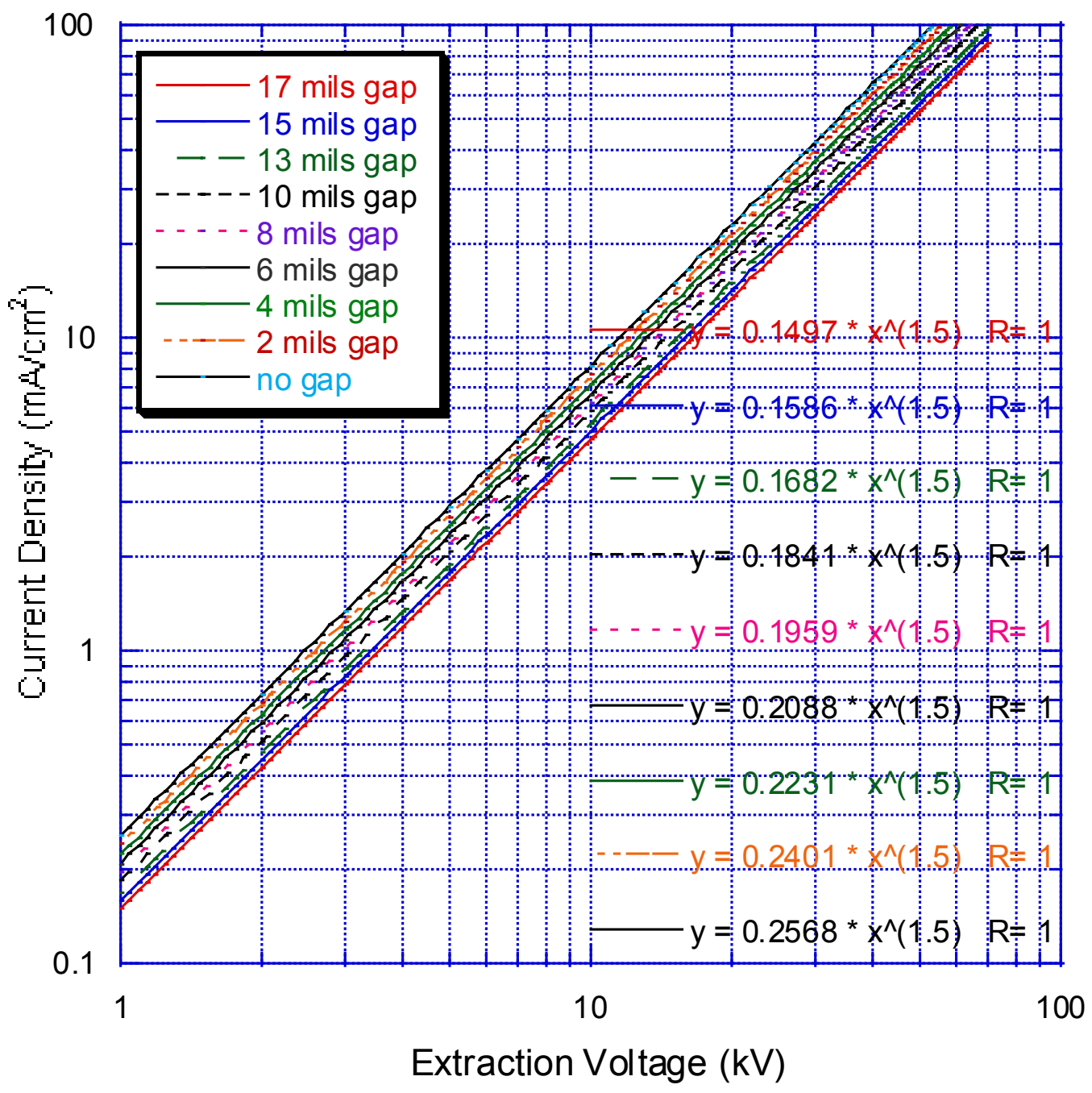

Figure 5.34. Plot of the expected $\mathrm{J}-\mathrm{V}$ characteristics and their dependence on the Pierce - emitter gap. 
According to the simulations, the total ion yield is affected mostly due to a change in the emitting profile of the ions. As the gap increases there is a decrease in the potential gradient that occurs mostly at the higher radii of the source. Given that the contribution to the total current is most significant at the outer radii, the effects become quite noticeable. Figure 5.35 shows a family of plots displaying the calculated beam profiles obtained from the simulations, again with the gap value as a parameter.

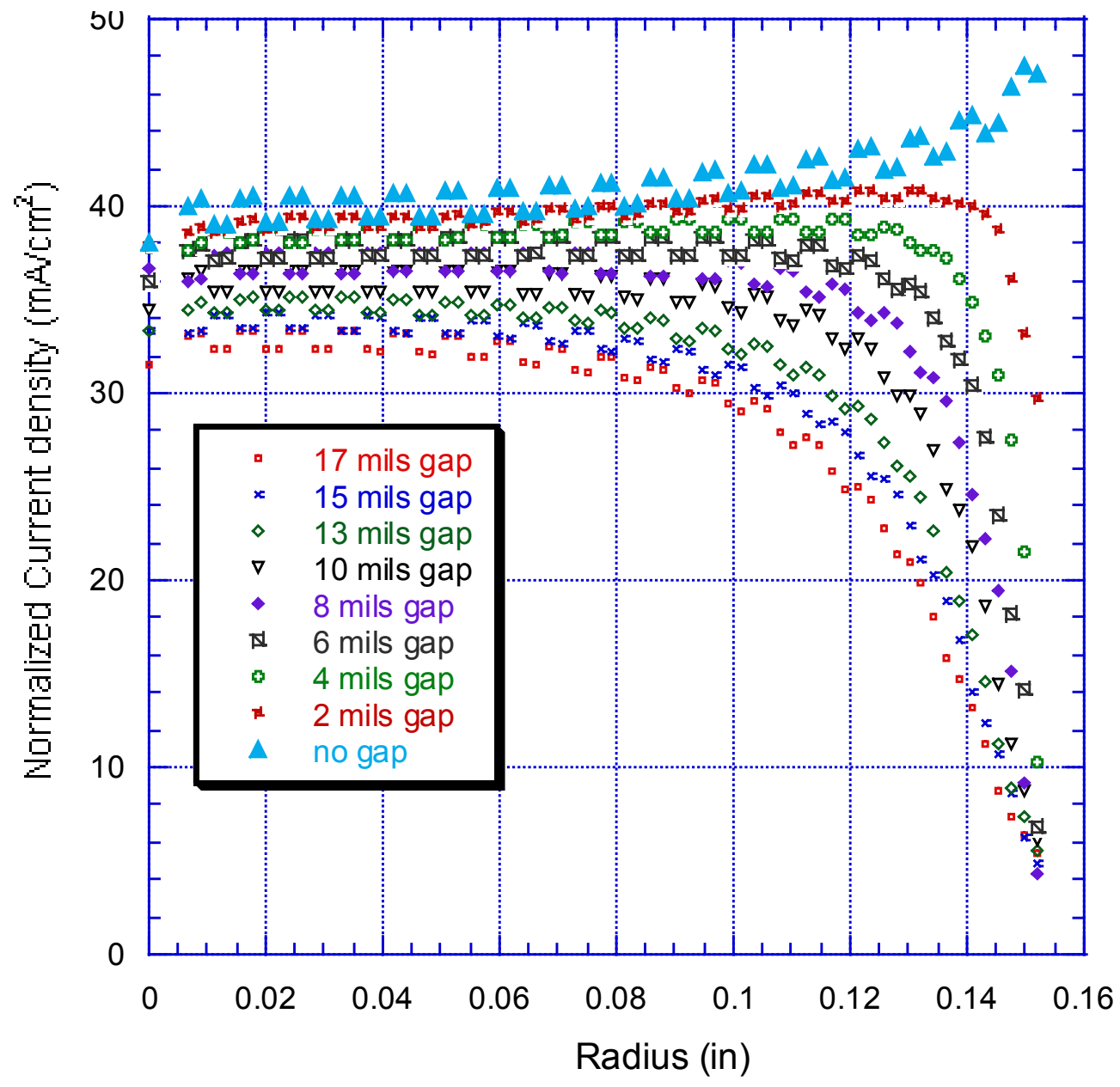

Figure 5.35. Track 5.0 modeling of the effect of the Pierce - emitter gap on the current density distribution as a function of emitter radius. 


\section{Chapter 6: Conclusions}

This section summarizes the main findings of the work presented in the previous pages. The reader is referred to the appropriate sections in the main text where the different items are treated in more detail.

\subsection{Potassium Aluminum Silicate emitters.}

In this work, a more extensive use of a Scanning Electron Microscope (SEM) was made as a means to have an improved quality control on the preparation of the potassium aluminosilicate emitters used in HIF experiments. The use of this diagnostic allowed for identifying the most desirable microscopic appearance and composition of the used materials and the acceptable methods of preparation. These diagnostics had a positive impact in the capability to produce potassium aluminosilicates capable of high ion yield as well as improved surface conditions. 
a. In accordance with what is expected from its physical properties, the mineral Leucite is considered to be the one responsible for the high current density yields observed.

b. To maintain the ideal (in the stoichiometric sense) composition of this material is difficult for three main reasons: Firstly, small fluctuations in the purity or amounts of the materials used for its fabrication will provoke the presence of different phases with slightly different compositions throughout the material. This may be partially supported by the observation of a changing signature (the characteristic X-ray spectra) of the used emitter materials. Second the high temperatures required for the preparation of the emitter do provoke the loss, at different rates, of the elements that compose the aluminosilicate. This is supported by the stronger changes in the X-ray signature observed as the preparation temperature of the emitter increases. The claim that the loss rate of the elements occurs at different rates is supported by the fact that the element potassium seems to be most strongly affected by this circumstance, as judged by the changes on the intensity of its characteristic peak. Thirdly, the composition of the aluminosilicate is itself a product of a delicate balance between the cations and anions that make up the material. It is to be expected that the fluctuations in the composition will result in the presence of different phases. The extraction of a high current density $\mathrm{K}^{+}$beam from such a structure is itself a cause for a composition change at the surface level of the material. It is not known for sure how the emitter reacts to such abrupt changes in surface composition, but it is reasonable to believe that by considerations of charge neutrality, some of the 
components of the negatively charged framework that enclose the cations will evaporate from the surface. This constitutes a continuous material loss mechanism. Evidence for the presence of different atomic groups has been observed in other experiments prior to this work (Tiefenback, 1986). The neutral particle detector used in this work measured neutral particle levels of the order of $\sim 0.5 \mu \mathrm{A} / \mathrm{cm}^{2}$ when pointed at an aluminosilicate emitter at $1,150{ }^{\circ} \mathrm{C}$. In combination with this process it is possible to expect a participation of diffusive mechanisms within the material, in a natural effort to compensate for the composition fluctuations, and aided by the relatively high temperature of operation of the ion sources.

c. The conjecture that there are diffusive processes of some relevance in the operation of aluminosilicate sources is partially supported by two observations. On one hand, the performance of composite materials made by combining tungsten or molybdenum powders with aluminosilicate powders never displayed a characteristic "activation" or improvement in its ion output with time. On the contrary, their output seemed to be characterized by a continual decay analogous to the diffusion-limited processes that are observed in the case of the doped contact ionization sources discussed in Chapter 5. Since the only clear difference between these aluminosilicates is the physical disposition in a collection of small ( $\sim$ micron size) grains as opposed to a single layer (as in the usual emitters), it is possible that the effective thinness of the emitter provides an extremely small "reservoir" of alkali atoms, that diffuse across the grain dimensions. While this current decay can also be explained by the continual material loss from the 
emitters, no appreciable change in the amount of aluminosilicate was observed after operation. The second observation is the behavior of an emitter "depleted" by the application of a DC field. The characteristic recovery of the emitter is also reminiscent of the behavior expected if there were a diffusive flow of ions towards the allegedly depleted surface.

d. Aluminosilicate emitters showed an unexpected robustness in their capability to provide sustained levels of high current density pulses in continual operation. The most recent designs considered for high J sources involve the use of smaller emitters than what were used in these experiments $(\sim 2 \mathrm{~mm}$ diameter as opposed to $\sim 5 \mathrm{~mm}$ ). The current extracted from these sources has been higher than in the mentioned designs by a factor of 3 . Nevertheless, the associated values of the normalized emittance have also been higher, but by a factor of $\sim 10$. It is very likely that acceptable parameters can be achieved with this type of source for the multiple beamlet injector configurations, based on the flexibility that has been identified (in simulation work) with respect to the initial beam emittance before merging. The aluminosilicate type of source is then a viable option for pursuing experimental work. The use of such type of source in a fusion driver configuration is questionable, since such application would imply high repetition rates that, combined with the apparent diffusion or recovery time (see section 4.2.4) would mean a relatively short lifetime before replacement or recovery is required. In contrast to the contact ionization type, there is no known way of providing continual replenishment in the case of the aluminosilicate source. 
e. By observing the X-ray spectra of the activated aluminosilicate sources (section 4.2.3), as well as the spectrum of the source that was depleted and then "reactivated", it can be seen how the high output yield of the aluminosilicate ion sources is correlated to a relatively low potassium content at the surface. This calls into question whether it is the actual leucite form of the mineral which is responsible for the maximum current density achievable, or if it is a slightly changed phase, that forms over time during operation. Only a more detailed crystallographic analysis could settle this matter.

\subsection{Contact Ionization Sources.}

a. High current densities have been verified in this work. The actual capability of these sources to attain these ion yields was not obvious despite being predicted by theory. The reason for this is that the theoretical framework that explains the behavior of the contact ionization sources was developed under experimental conditions of steady state and constant surface coverage that are not readily reproducible in the more dynamic conditions of the HIF sources. This was the basic justification for the efforts described on these pages. Two main reasons can be offered for the not attaining the desired level of current density of $100 \mathrm{~mA} / \mathrm{cm}^{2}$ of potassium equivalent. First, it became evident that the effect of the emitterPierce electrode gap has a more significant effect on the ion output than in larger ion sources (see Section 5.8.1). More relative surface area belongs to the "edge" of the emitter and is thus more significantly affected. The second factor is related with the dependence of the ion output with respect to the surface coverage. As 
higher currents are demanded, the range of values of the surface coverage that are capable of supporting these higher current densities is reduced. Since the actual value of the coverage will change more rapidly as the temperature increases, and since the higher temperatures are required for the higher current yields, this makes the optimum coverage value a moving target that can not be maintained for prolonged periods of time. While the highest $<\mathrm{J}>$ value observed was 90 $\mathrm{mA} / \mathrm{cm}^{2}$, more typical values easily attainable with the applied voltages (of up to $70 \mathrm{kV}$ ) were $\sim 75 \mathrm{~mA} / \mathrm{cm}^{2}$.

b. The previous item indicates that in order for a contact ionization source to be usable in a sustained manner in HIF experiments, at the desired levels of high current density, methods of continual surface replenishment must be used. It has been shown, though, that the required flow rates needed to maintain an adequate surface coverage level are very low. This means that it is not necessary to reproduce the same methods of surface replenishment used in other applications such as the ion thruster systems. As mentioned in the text, these systems require much higher alkali fluxes, accompanied by higher neutral desorption with all its associated problems. It has been shown that the low levels of alkali coverage required will not need a neutral-particle diffusion dominated regime proper of high alkali content in the porous substrate. Since only much smaller alkali content is required, the dominating diffusion mechanism will be the surface migration of ions. This would provide a slow but steady replenishment of the emitter surface capable of sustaining moderate repetition rates. 
c. The mode of operation mentioned in item b. will be appropriate for operation at low duty factors, as required by usual HIF experiments. A driver mode of operation will require higher fluxes and thus will operate almost in a different mode, with higher neutral content in the substrate, faster diffusion and higher neutral particle desorption rates, in a mode closer to the regime of the ion thrusters.

d. The estimates of the diffusion constants made in this work, as well as the identification of the different modes in which such emitters may operate, opens new possibilities at the simulation level. It is possible to carry out a more detailed, 2 dimensional simulation of what occurs in doped ion sources regarding the diffusion processes. This is relevant for both, the small emitters used in these experiments, as well as for the larger ion sources used in other experiments. In principle, it would be possible to simulate, given an initial content of alkali dopant, where the gradients of the concentration are the highest, where there are more losses of alkalis and where is a source most likely to show signs of depletion during operation. Given that the diffusion of the particles within the substrate is not fast, it is reasonable to expect that non-uniformities in the initial distribution of alkalis (due to poor doping procedures), may actually play a role in the performance of the source and on its lifetime (as would be the case of an insufficient penetration of the dopants). Therefore, these results may point to new directions of improving the quality of the ion sources of this type commonly used.

e. The estimates made in this work regarding the diffusion constants, and the subsequent elaborations on the maximum repetition rates achievable are very 
rough and perhaps describable as the most simple minded approaches that will still lead to reasonable results. These analyses point at directions of research that, to the author's knowledge and opinion, have not been properly addressed before and have the potential of becoming important issues as the ion demand and repetition rate on this type of ion sources is increased in future experiments. It seems very logical to expect that the maximum repetition rate deliverable by an ion source working on the mode described in these pages will depend on the diffusion-limited rate of replenishment of the surface, and so that there is a natural limit to this repetition rate. This work constitutes a first look into issues of this sort.

\subsection{General Issues.}

a. Small fluctuations or changes in the position of the emitter within the Pierce produce relatively large effects, given the more reduced dimensions of the high $\mathrm{J}$ ion guns, and the fact that the current scales as the inverse of the square of the extractor - emitter gap.

b. Issues such as the Pierce-emitter gap, and the changes in position of the emitter seem to be automatically addressed by other type of ion sources such as the plasma ion source or MEVVA type source. The preliminary experiments described in the appendices also open research possibilities into promising ways to deliver the required beam parameters for the multiple-beamlet architectures in HIF drivers. The experiments described are not exhaustive and it is expected that 
additional work carried out will strengthen the case of these ion sources as perhaps the most appropriate candidates for this type of configuration. 


\section{BIBLIOGRAPHY}

Anderson, O. Emittance growth from merging arrays of round beamlets. Fusion Engineering and Design 32-33 (1996). 209-217.

Brewer. G.R. Ion Propulsion: Technology and Applications. Gordon and Breach Science Publishers. 1970.

Carey, Van P. Liquid-Vapor Phase-Change Phenomena. An introduction to the thermophysics of vaporization and condensation processes in heat transfer equipment. Hemisphere Publishing Corporation. 1992.

Cho, A.Y. and Shelton, H. Cesium Neutral and Ion Emission from Carburized and Oxygenated Porous Tungsten. AIAA Journal. Vol. 2, No. 12, December 1964.

Chupp, W.W., et al. HIFAR Note \#387: Preparation of Large Area Thermionic Positive Ion Emitters. 1993.

Cubic leucite structure was obtained from the ICSD (Inorganic Crystal Structure Database) database and viewed with the Cosmo Player 2.1.1.

Comsa, G. The Dynamical parameters of Desorbing Molecules. (in Dynamics of GasSurface Interaction G. Benedek and U. Valbusa Editors. Springer Verlag Series in Chemical Physics 21. Proceedings of the International School on Material Science and Technology, Erice, Italy, July 1-15 1981.)

Chacon-Golcher, E. Development of High Current Density Surface Ionization Sources for Heavy Ion Fusion. Rev. Sci. Instrum. Vol. 73. No. 2. Feb. 2002. pp. 10361038.

Cunningham, R.E. and Williams, R.J. Diffusion in Gases and Porous Media. Plenum Press, New York. 1980.

de Boer, J.H., The Dynamical Character of Adsorption. Oxford - Clarendon Press. 1968. $2^{\text {nd }}$ edition.

Evans, R.C. The Positive Ion Work Function of Tungsten for the Alkali Metals. Proc. Roy. Soc. (London) A139. pp. 604 - 617. (1933)

Feeney, R.K. et al. Aluminosilicate sources of positive ion for use in collision experiments. Rev. Sci. Instrum., Vol. 47, No. 8, Aug. 1976.

Forrester, T.A., Large Ion Beams, Fundamentals of Generation and Propagation. John Wiley \& Sons, 1988. 
General mineral information was obtained from: $\underline{\mathrm{http}: / / \text { mineral.galleries.com }}$

Grote, D.P., et al. Design and Simulation of the Multi-Beamlet Injector for a High Current Accelerator. Submitted for publication.

Hughes, D.W. et al. Aluminosilicate-composite type ion source of alkali ions. Rev. Sci. Instrum. 51(11), Nov. 1980. p. 1471.

Husmann, O.K., et al. Influence of Residual Gas Atmosphere in Space Chambers on Neutral Efflux and Critical Temperature of Tungsten Ionizers. AIAA Journal. Vol. 4, No. 2, February 1966.

Incropera, F.P. and DeWitt, D.P. Fundamentals of Heat and Mass Transfer. $5^{\text {th }}$ Edition. John Wiley \& Sons. 2002.

Kwan, J.W., et al. Development of high current density surface ionization sources for Heavy Ion Fusion. Proceedings of the Particle Accelerator Conference. 2001.

Kwan, J.W. et al., High Current Density Ion Sources for Heavy Ion Fusion Accelerators. Proc. Part. Accel. Conf., Vancouver, May, (1997) p.2755

Kwan, J.W., et al. In Proceedings of the Particle Accelerator Conference. 2001.

Lima-de-Faria, J. Structural Mineralogy, an Introduction. Kluwer Academic Publishers. 1994.

MacLaren, S. A Scaled Final Focus Experiment for Heavy Ion Fusion. Ph.D. Thesis. Physics Department, University of California at Berkeley. 2000.

Mark, H. and Verwey, E.J.W., editors. Advances in Colloid Science. Volume III. Interscience Publishers, Inc. 1950.

Ong, P.P., et al. Aluminosilicate sources of Cs+ ions. Rev. Sci. Instrum. 65 (12), December 1994. Page 3729.

Pargellis, A.N., et al. Thermionic emission of alkali ions from zeolites. J. Appl. Phys. 49(9), September 1978. pp 4933.

Reid, R. et al. The Properties of Gases and Liquids. $4^{\text {th }}$ Ed. McGraw-Hill International Editors. 1988.

Scheer, M.D. and Fine, J. Kinetics of Cs+ Desorption from Tungsten. The Journal of Chemical Physics. Vol. 37, No. 1. July 1962. 
Tiefenback, M.G. Space-Charge Limits on the Transport of Ion Beams in a Long Alternating gradient system. Ph.D. Thesis. University of California at Berkeley. November 1986.

Taylor, J.B. and Langmuir, I. The Evaporation of Atoms, Ions and Electrons from Cesium Films on Tungsten. The Physical Review. Vol. 44, No. 6. September 1933. Page 423.

Wiggs, P. The Relation between Gas Permeability and Pore Structure of Solids. In Structure and Properties of Porous Materials. Everett, P. and Stone, T., editors. Academic Press, NY. 1958. 


\title{
APPENDIX 1: INITIAL EXPERIMENTS OF RF GAS PLASMA SOURCE FOR HEAVY ION FUSION *
}

L. Ahle, R. P. Hall, A.W. Molvik, LLNL, Livermore, CA 94550, USA

E. Chacon-Go1cher, J. W. Kwan, K, N. Leung, J. Reijonen, LBNL, Berkeley, CA 94720, USA

\begin{abstract}
The Source Injector Program for the U.S. Heavy Ion Fusion Virtual National Laboratory is currently exploring the feasibility of using RF gas plasma sources for a HIF driver. This source technology is presently the leading candidate for the multiple aperture concept, in which bright millimeter size beamlets are extracted and accelerated electrostatically up to $1 \mathrm{MeV}$ before the beamlets are allowed to merge and form $1 \mathrm{~A}$ beams. Initial experiments have successfully demonstrated simultaneously high current density, $\sim 100 \mathrm{~mA} / \mathrm{cm}^{2}$, and fast turn on, $\sim 1 \mu \mathrm{s}$. These experiments were also used to explore operating ranges for pressure and RF power. Results from these experiments are presented as well as progress and plans for the next set of experiments for these sources.
\end{abstract}

\section{BEAMLET INJECTOR FOR HIF}

The source injector for a heavy ion fusion driver must deliver $\sim 10^{16}$ ions to the accelerator [1]. Given current limits of sources and transport limits of accelerators, the injector will almost assuredly have multiple beams. Most present conceptual designs have an injector energy of $1.6 \mathrm{MeV}$ and current per beam of $0.5 \mathrm{~A}$ [2]. Presently, the Heavy Ion Fusion Virtual National Laboratory is pursuing a concept of creating the $0.5 \mathrm{~A}$ beams 
by initial extracting many, $\sim 100$, small beamlets and accelerating them to $1 \mathrm{MeV}$ before they are allowed to merge to together [3]. This concept promises to produce brighter, a key figure of merit, higher current beams and a more compact injector. Initial simulations have given encouraging results.

Another advantage of the beamlet architecture is the beam brightness of the merged beam is dominated by the emittance growth of the merging process and, is only a weak function of the ion temperature of the source. This allows the possibility of using sources with higher ion temperatures instead of surface ionization sources. The merged beams in the beamlet injector only achieve high brightness if the current density of the beamlet is high, $\sim 100 \mathrm{~mA} / \mathrm{cm}^{2}$. In fact, current density becomes a more important criterion for the source than ion temperature.

\section{RF PLASMA SOURCE}

For the beamlet concept to be practical, there must be a source that delivers high current density and easily accommodates the geometry of many millimeter size beams. A plasma source is such a source. Specifically, the HIF program has started development of a multicusp RF plasma source for HIF. These sources have been used to produce high current density beams and can form the beamlets by using a single RF bucket with a grid for the extraction plate. In fact, K.N. Leung's group at LBNL demonstrated over 100 $\mathrm{mA} / \mathrm{cm} 2$ for Ar. [4].

Another important requirement for the sources in HIF is the ability of a fast turn on. To minimize non-linear effects in the head of the beam, the rise time of the voltage waveform across the first gap must be $\sim 100$ ns. Plasma formation times are usually of 
the order $10 \mu \mathrm{s}$, implying the necessity for a fast high voltage pulser. Even with such a pulser, a fast rise time is not guaranteed. The emission surface in a plasma source does change as the voltage pulse ramps up, so a fast beam current pulse may not arise with a fast high voltage pulse. K.N. Leung's group also demonstrated fast rise time, - $1 \mu \mathrm{s}$, with an RF plasma source, but with only a few $\mu \mathrm{A}$ of beam current [5]. Thus, HIF source injector program recently conducted experiments aimed at simultaneously demonstrating fast rise time and high current density.

\section{EXPERIMENTAL SETUP}

The experiments were preformed with the same $10 \mathrm{~cm}$ multicusp source used in reference [4]. The plasma chamber has an inner diameter of $10 \mathrm{~cm}$ with $20 \mathrm{SmCo}$ magnet columns. A 1.5-turn, quartz antenna with a $5 \mathrm{~cm}$ diameter coil generates plasma inside the chamber. The power is delivered to the antenna from a RF amplifier via a fifty-ohm transmission line and an impedance matching network. The RF amplifier generates a 2 ms burst of $13.56 \mathrm{MHz}$ RF signal at a rep rate of $10 \mathrm{~Hz}$. The maximum peak RF output power of the amplifier is $5 \mathrm{~kW}$. The gas, Argon, is introduced to the plasma chamber through a needle valve and the absolute pressure is measure by a capacitance manometer. A small tungsten starter filament is used to feed seed electrons and typically drew about $60 \mathrm{~W}$ of power in order to provide an adequate source of electrons.

The extraction system consists of a plasma electrode with an aperture of $3 \mathrm{~mm}$. The gap to the ground electrode is $3 \mathrm{~mm}$, which has a $4 \mathrm{~mm}$ diameter opening for the beam to travel through. A pulser, manufactured by Diversified Electronics, provides the extraction voltage. The system is capable of delivering a $50-\mathrm{kV}, 20$ microsecond pulse with a peak 
current of 25 A. A faraday cup with a magnetic filter was used to measure the beam current extracted from the source. The faraday cup picks up transients from the extraction pulser. These transients are subtracted from the data in software by recording the signal from the faraday cup with the extraction voltage pulse but without the RF amplifier so that no beam is extracted. Figure 1 shows a raw faraday cup signal with and without the RF amplifier running and the software subtraction of the two. As can be seen from the figure, the transients picked up by the faraday cup is only a small fraction of the signal.

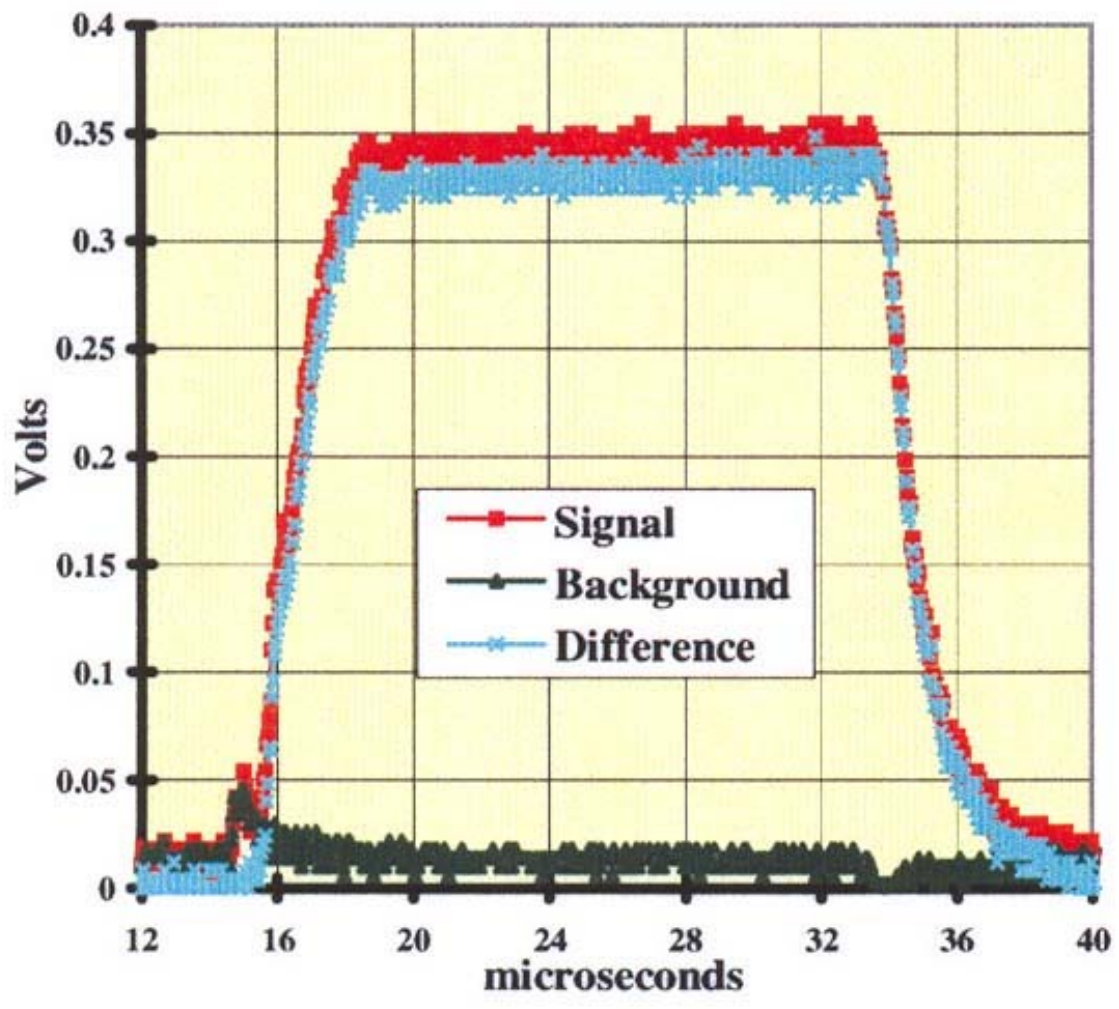

Figure 1: Sample signal from Faraday cup with and without beam extraction and difference between the two.

\section{EXPERIMENTAL RESULTS}

First, it was verified that this source could produce $100 \mathrm{~mA} / \mathrm{cm}^{2}$ of beam current density. The matched condition for the source configuration was determined by varying the extraction voltage and measure the flattop beam current. Figure 2 shows a plot of 
current density versus extraction voltage. The data shows a typical voltage to the threehalves power behavior for low voltage. In this region, the Child-Langmuir limit for space charge limited ion extraction is lower than the emission limit from the plasma source. At around $15 \mathrm{kV}$, the two limits are near equal. Above, this voltage, the extracted current density plateaus, indicating emission limited beam extraction. For this example, the matched condition was determined to be $14.4 \mathrm{kV}$. This plot clearly shows that 100 $\mathrm{mA} / \mathrm{cm}^{2}$ can be produced by this source.

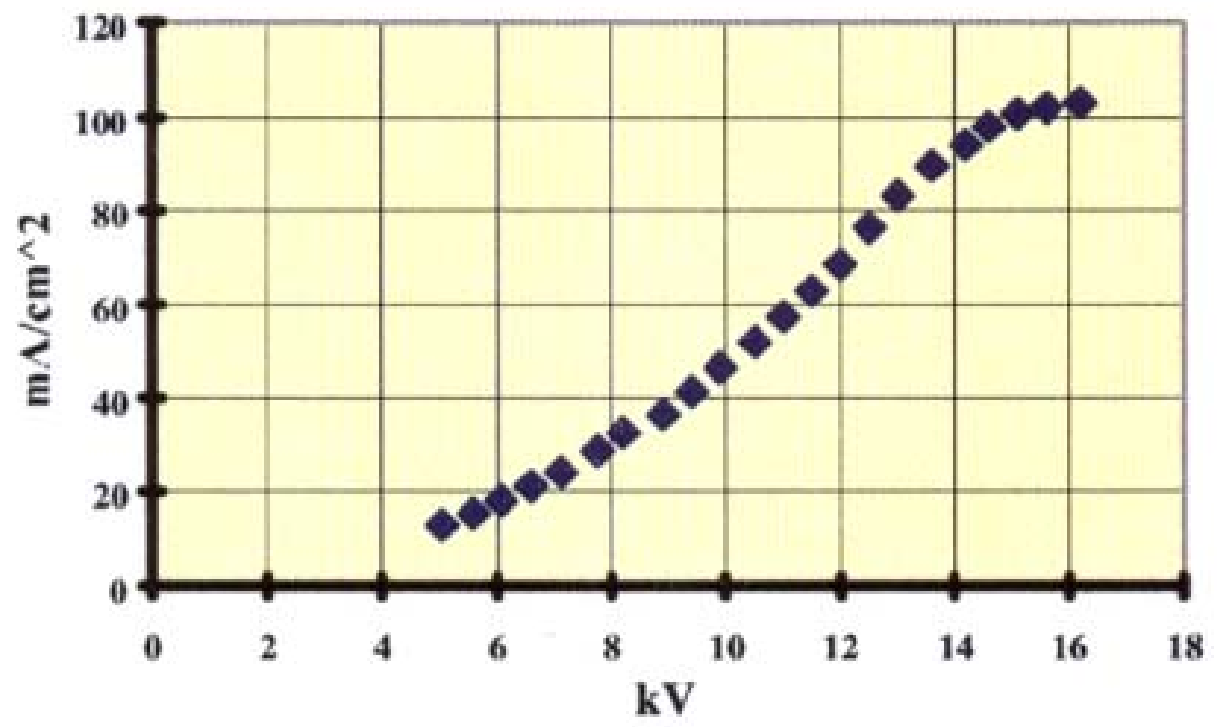

Figure 2: Current density versus extraction voltage.

With the starter filament in place, 2 mtorr was found to be the minimum pressure for stable operation of the source. Below this pressure, the RF pulse did not always form plasma. Below about 1.0 mtorr, plasma was never ignited. The RF amplifier was set to 3 $\mathrm{kW}$ of peak power for the $2 \mathrm{~ms}$ pulse. The extraction pulser was set to fire $500 \mu \mathrm{s}$ after the RF pulse started in order to allow the plasma to stabilize before beam extraction. The extraction voltage was set to $14.4 \mathrm{kV}$. 
Figure 3 shows the resulting extraction voltage waveform and extracted current density. The delay between the two is the time of flight of the argon ions to the Faraday cup. The measure current density is $93.3 \mathrm{~mA} / \mathrm{cm}^{2}$. This, however, is not a fundamental limit of the setup, but simply the matched condition for this RF power setting and pressure. Figure 4 is the same plot with the time axis expanded around the front of the pulse. Analysis of the waveforms revealed a $2.2 \pm 0.1 \mu$ s rise time for $10 \%-90 \%$ of the flattop. The extraction pulse also has a similar rise time, $2.3 \pm 0.1 \mu \mathrm{s}$, indicating that the rise time is being limit by the pulser and not the plasma source itself.

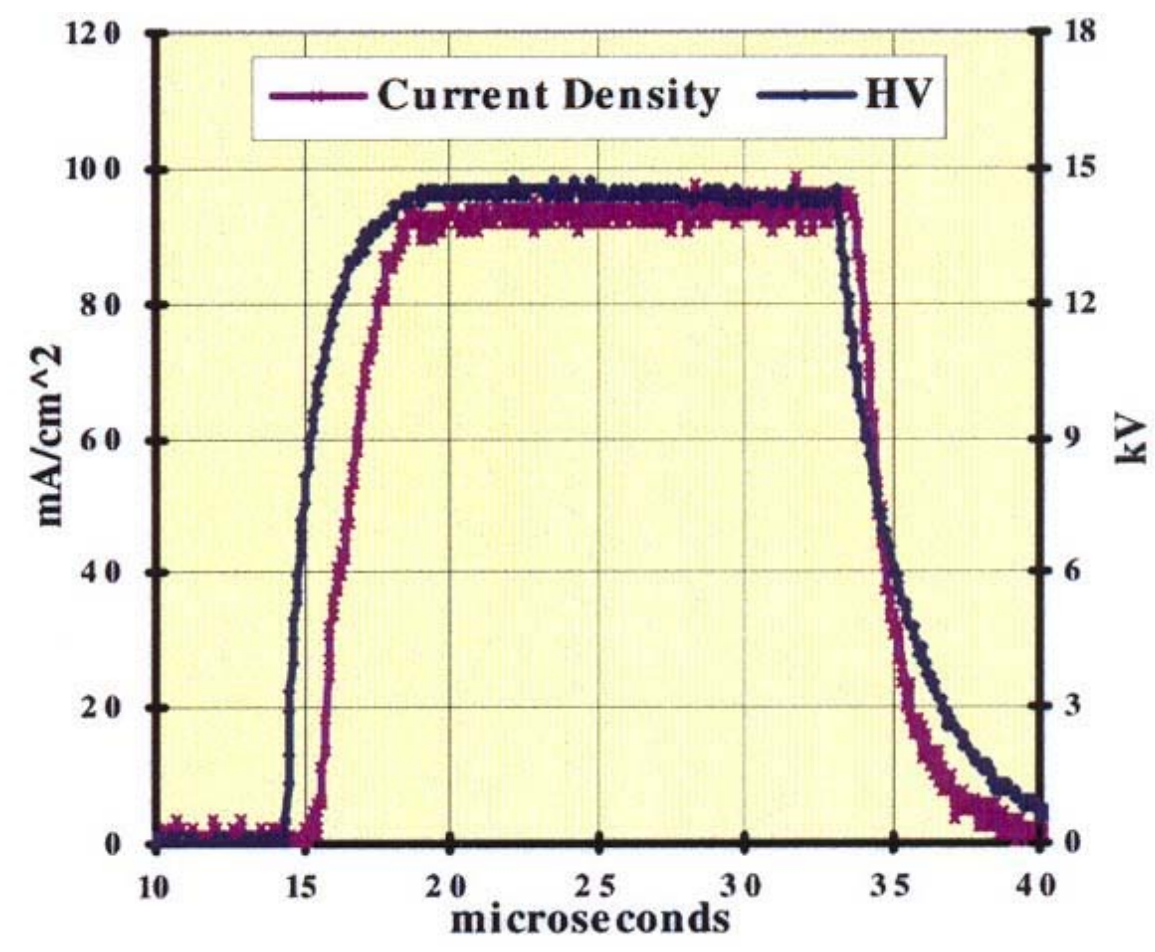

Figure 3: High voltage pulse and extracted current pulse for plasma source with starter filament. 


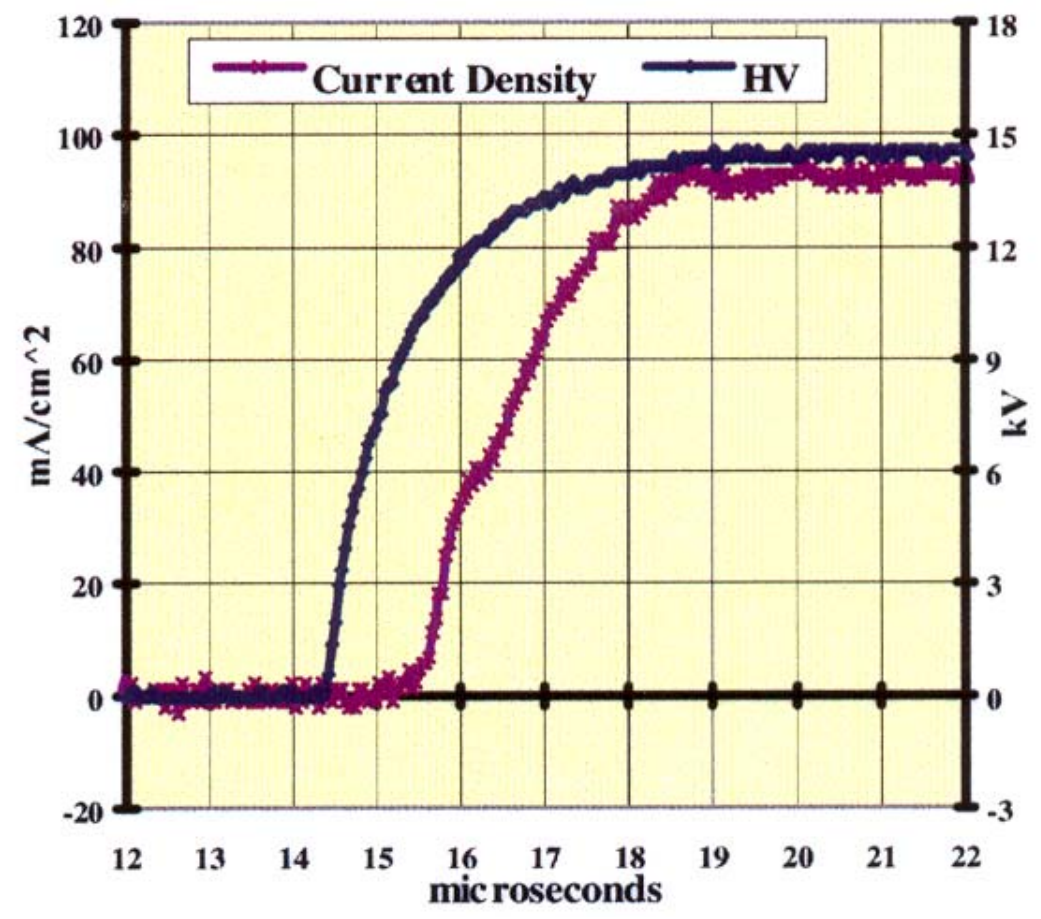

Figure 4: Same as figure 3, only with the time scale expanded around start of pulse.

The rise time of the pulser is dominated by the stray capacitance and inductance of the system. One way to significantly reduce the effect of the load is to remove the starter filament and the isolation transformer needed to deliver power to it. This was removed from the system and the measurement repeated. Unfortunately, without the starter filament the minimum pressure for stable operation is 18 mtorr. To get a similar current density, the RF power was reduced to $2 \mathrm{~kW}$. For these settings, a matched condition was achieved at $15.7 \mathrm{kV}$. Figure 5 shows the resulting waveforms. For this configuration the rise time of the extraction pulse is $1.4 \pm 0.1 \mu \mathrm{s}$. The current pulse again appears to follow the extraction pulse with a rise time of $1.5 \pm 0.1 \mu \mathrm{s}$. Again, there is no evidence the plasma source itself is limiting the rise time. Unfortunately, further reduction in the rise time of the extraction pulse would require a different pulser or major changes to the test setup. 


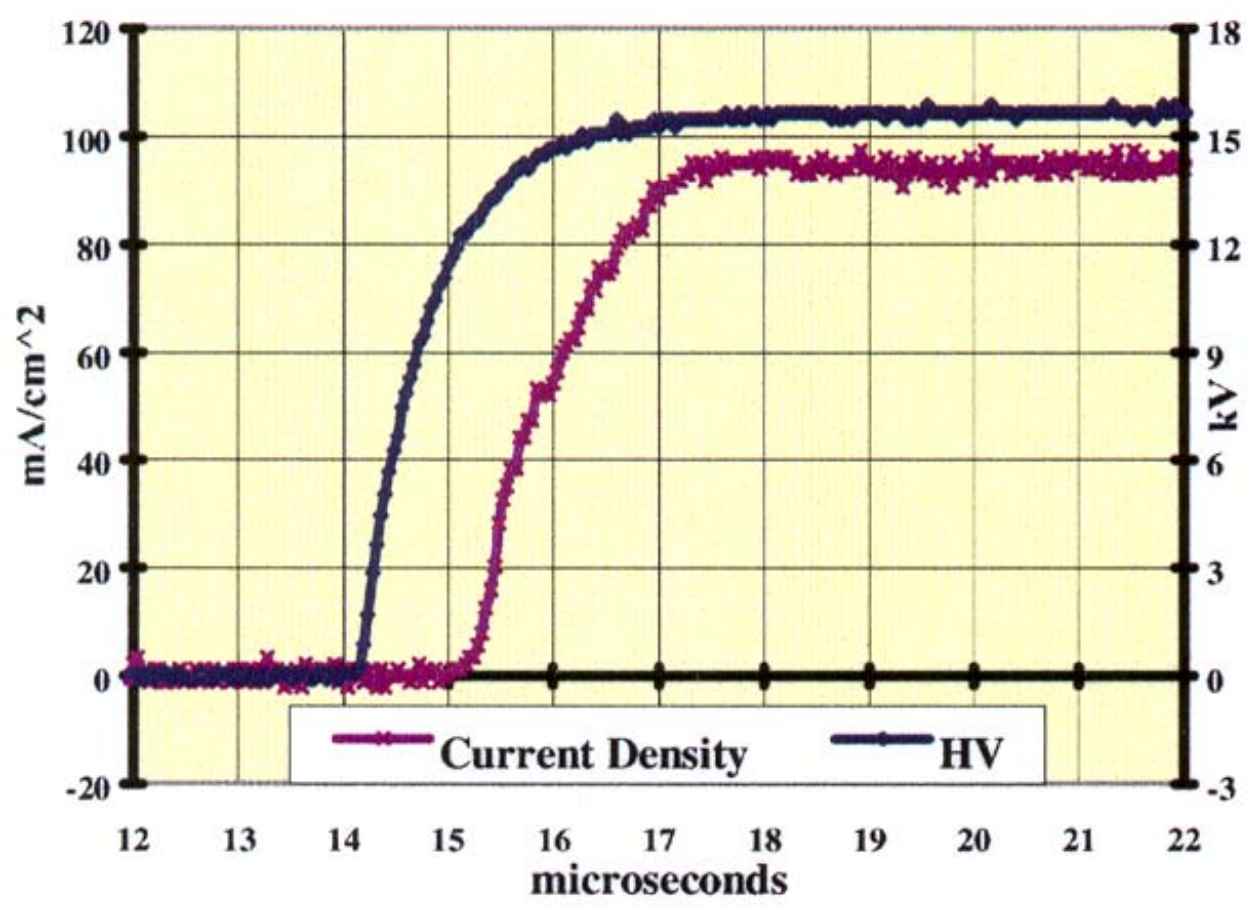

Figure 5: Extraction voltage and current density versus time for plasma source without the starter filament.

\section{DISCUSSION}

These results demonstrate it is possible to achieve simultaneously high current density, $\sim 100 \mathrm{~mA} / \mathrm{cm}^{2}$, and fast rise time, $\sim 1 \mu \mathrm{s}$, with a multicusp RF gas plasma source. The data indicate the rise time is limited by the extraction pulse and not the plasma source itself. Though there may be a plasma source limit below $1 \mu$ s. This result is achieved by fast switching of the extraction voltage and not the RF power. A starter filament allows for operation at a lower pressure, $2 \mathrm{mtorr}$ as compared to $18 \mathrm{mtorr}$ without. Also only $3 \mathrm{~kW}$ of peak RF power is needed to generate current densities of 100 $\mathrm{mA} / \mathrm{cm}^{2}$. Even though more power would be needed for a source large enough to 
generate $100,5 \mathrm{~mA}$ beamlets, a duty factor of less than $0.1 \%$ is needed for the RF power, which implies a low average power system is needed.

\section{FUTURE PLANS}

Based on these results, the HIF program has decided to continue development of the plasma source. A larger plasma source capable of delivering 100, $5 \mathrm{~mA}$ beamlets is under construction. The inner diameter of the plasma cavity is $26 \mathrm{~cm}$ and it has 38 magnets surrounding the outside. The antenna is a one and two-thirds turn, quartz antenna with a diameter of $10 \mathrm{~cm}$. Based on the results of the previous source, it is estimated that $\sim 10 \mathrm{~kW}$ of peak RF power is need to create plasma with a high enough density to extract $100 \mathrm{~mA} / \mathrm{cm}^{2}$. This RF power will be provided by a pulsed RF system, which can be floated at high voltage.

The source will be tested on the source pulser system of the Recirculator [6]. This pulser system is capable of delivering up to $100 \mathrm{kV}$ and rise times of $\sim 300 \mathrm{~ns}$ when driving a $10 \mathrm{nF}$ load. This system may be capable of even faster rise times for loads with less capacitance, as is expected when this source is connected to this platform. This pulser system should allow full scale testing for the first gap of the multi-beamlet architecture. Experiments will be focused on exploring extracted current density and emittance versus RF power and gas pressure. Charge exchange in the extraction gap is an important concern and energy measurements will be performed to determine the magnitude of this effect. Also, charge state purity will be explored with time of flight measurements. A pulsed gas system may also be developed to minimize gas load and 
thus charge exchange in the extraction gap. Results from the experiments should be available by April of 2002 .

\section{REFERENCES}

[1] R. O. Bangerter, Nuovo Cimento I06A (11), 1445 (1993).

[2] J. W. Kwan, et. aI., Nuovo Cimento 1O6A (11), 1541, (1993).

[3] J. W. Kwan, et. aI., "Design of a Compact High Current Injector for Heavy Ion Fusion", these proceedings.

[4] 1. Reijonen, et. aI., Proceedings of 1999 Particle Accelerator Conference, 1943, (1999).

[5] L. T. Perkins, et. aI., Review of Scientific Instruments, 69 (2), 1060, (1998).

[6] L. Ahle, et. al., Proceedings of 1999 Particle Accelerator Conference, 3248, (1999).

*This work performed under the auspices of US DOE by UC-LLNL under contract W-740S-ENG-48 and by UC-LBNL under contract DEACO3- 76SFOO098. 


\section{APPENDIX 2: $\quad$ Time resolved emittance of a bismuth ion beam from a pulsed vacuum arc ion source}

André Anders and Edwin Chacon-Golcher

Lawrence Berkeley National Laboratory, University of California,

1 Cyclotron Road, Berkeley, California 94720

\section{Abstract}

The emittance of a pulsed bismuth vacuum arc ion source has been measured using a time-resolving double slit emittance scanner. Each arc pulse was $250 \mu$ s long. Ion extraction was performed with $20 \mu$ s pulses $200 \mu$ s after arc triggering, when $\mathrm{Bi}$ ions are almost exclusively singly charged. This was motivated by a possible use of the ion source for heavy ion fusion. Pulsed extraction implies perveance mismatch during extractor voltage rise and fall time. The emittance increased with arc current. The beam current is very constant when operating in the slightly overdense plasma regime because fluctuations of the ion supply current are partially offset by extraction optics. For an extraction voltage of $35 \mathrm{kV}$, a bismuth current density of up to $18 \mathrm{~mA} / \mathrm{cm}^{2}$ with a normalized emittance of $0.006 \pi \mathrm{mm}$ mrad was obtained for a beamlet of $2 \mathrm{~mm}$ nominal diameter.

Vacuum arc ion sources, also known as metal vapor vacuum arc (Mevva) ion sources, can deliver ion beams of high-current density of virtually all solid conducting materials ${ }^{1}$. Ions are extracted from fast streaming, high-density metal plasma that originates at cathode spots ${ }^{2}$. In the present experimental setup, ion extraction is accomplished using three multiple-aperture grids of the common acceleration-deceleration configuration ${ }^{3,4}$. 
Vacuum arc ion sources can be used, for instance, for large area metal ion implantation. For more than a decade, they have also been considered for heavy ion fusion ${ }^{5-7}$ (HIF). However, vacuum arc plasmas are rapidly fluctuating due to explosive nature of plasma generation at cathode spots. There have been attempts to control plasma flow and to suppress plasma and beam noise, e.g., by biased meshes introduced in the plasma prior to ion extraction ${ }^{6-10}$. With the progress achieved, the vacuum arc ion source remained a candidate for heavy ion fusion applications. Despite a considerable body of work, no time-resolved emittance measurements have been reported. To further evaluate and improve the viability of this kind of ion source, time-resolved emittance measurements are critical.

For the measurements, the Berkeley Lab vacuum arc ion source "Mevva V", Ref. ${ }^{1}$, was equipped with a double slit emittance scanner (Fig. 1). Each slit had a width of $93 \mu \mathrm{m}$ (as measured in a scanning electron microscope) and a length of $50 \mathrm{~mm}$. The first slit was located $10 \mathrm{~mm}$ after the last, grounded extraction gird, and the plane of the second slit was $52.3 \mathrm{~mm}$ after the plane of the first slit. Both slits were moved with computercontrolled stepper motors coupled to Huntington actuators. The minimum step achievable was $2.5 \mu \mathrm{m}$. A Faraday cup was mounted behind the second slit; its output signal was amplified by a calibrated wide-bandwidth amplifier before the signal was recorded via a digital storage oscilloscope and data acquisition system. Data visualization and evaluation was accomplished by custom MatLab® software. 
We have selected bismuth as the metal of choice because (i) it is the heaviest nonradioactive metal, (ii) the charge state distribution can be shifted to almost $1^{+}$only, (iii) natural bismuth contains more than $99.9 \%$ of the isotope 209 , i.e. it is practically isotopically pure, (iv) its cost is relatively low, and (v) it is nontoxic compared to the neighboring elements $\mathrm{Hg}$ and $\mathrm{Pb}$. The ion charge state distribution contains almost only singly charged ions if the arc pulse is longer than $200 \mu$ s. Therefore, even when the ion pulse length of interest for HIF is about $20 \mu \mathrm{s}$, it was opted to use long arc pulses of 250 $\mu \mathrm{s}$ and pulse the ion extraction system $200 \mu$ s after arc triggering.

High voltage pulses for ion extraction were provided by a $50 \mathrm{kV}$ pulse modulator of 2.0 $\mathrm{k} \Omega$ impedance. Because the voltage at the load at impendance-matched conditions would be only $25 \mathrm{kV}$, we chose to operate a higher voltage, which can be obtained with a load resistor of e.g. $20 \mathrm{k} \Omega$, compromising the ideally rectangular voltage pulse shape. The constant voltage portion of each pulse is $15 \mu \mathrm{s}$ long. The large rise $(\sim 5 \mu \mathrm{s})$ and fall time $(\sim 15 \mu \mathrm{s})$ allow us to study the head and tail development of the beam pulse, although for later application one would try to operate a pulse modulator of higher voltage under impedance-matched conditions, thereby minimizing rise and fall time.

A three-grid extractor of the common accel-decel type was used. The diameter of the plasma grid hole was $2.0 \mathrm{~mm}$ and the diameters of the holes in the negative suppressor and grounded outlet grids were $4.0 \mathrm{~mm}$ each. 
Figure 2 shows a profile composite taken with 65 different positions of the slit with the Faraday cup. Corresponding to the fall and rise time of the extractor voltage pulse, one can clearly see the pulsed beam's head and tail, characterized by large divergence. The central section of the beam shows much lower divergence and associated lower emittance, see Fig. 3. In the following we focus on the properties of the central section. The emittance of the beamlet can be displayed in the $\mathrm{x}-\mathrm{x}^{\prime}$ phase space. For better

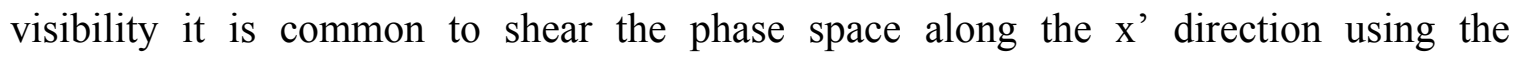
transformation $x_{\text {sheared }}^{\prime}=x_{\text {original }}^{\prime}-x\left\langle x \cdot x_{\text {original }}^{\prime}\right\rangle /\left\langle x^{2}\right\rangle$, where the \langle\rangle brackets indicate RMS averaging. An example is shown in Fig. 4.

It is known that the emittance is a function of the plasma conditions, and the easiest way of adjusting vacuum arc plasma parameters is via the arc discharge current. As anticipated, and confirmed by the data shown in Fig. 5, the total ion current and the emittance grow with increasing arc current. Data in Fig.5 refer to values in the central part of each pulse and exclude emittance during rise and fall time of the extraction pulse.

The main motivation for this work has been to improve the viability of a Mevva-type ion source for HIF applications. An extraction design analogous to the one used here aims at values per beamlet of current $I_{i}$ and normalized emittance $\varepsilon_{n}=\beta \gamma 4 \varepsilon_{r m s}$ for a $\mathrm{K}^{+}$ion beam of $\sim 5 \mathrm{~mA}$ and $0.002 \pi-\mathrm{mm}-\mathrm{mrad}$, respectively, at an extraction voltage of $100 \mathrm{kV}$; $\beta=v / c$ and $\gamma=1 / \sqrt{1-\beta^{2}}$ are the relativistic factors, $\varepsilon_{r m s}=\sqrt{\left\langle x^{2}\right\rangle\left\langle\left(x^{\prime}\right)^{2}\right\rangle-\left\langle x \cdot x^{\prime}\right\rangle^{2}}$ is the RMS emittance. At the lower extraction voltages and higher ion mass used here, the equivalent current is $\sim 0.55 \mathrm{~mA}$ (at $35 \mathrm{kV}$ ), that is, at the regime where the measured 
values were. The emittance values of up to $0.006 \pi$-mm-mrad are much smaller than previous data ${ }^{5,7}$ where a larger beam diameter used and where measurements were averaged over the beam pulse duration. The emittance determined here is still a few times the theoretically desired value. However, our experimental values are still acceptable for HIF applications because the individual beamlets will be merged, and the final emittance is relatively insensitive to the initial emittance per beamlet.

The quality of an ion beam in terms of ion current density and emittance critically depends on the position and shape of the boundary between quasi-neutral plasma and space charge in the extraction system. It is known that the current density of ions supplied to the plasma boundary needs to be well matched to the solution of the unipolar space charge problem, also known as perveance matching condition ${ }^{4}$. The unipolar space charge problem describes the limitation of charged particle flow by its space charge as governed by the Poisson equation. Child and later Langmuir solved this problem for the infinite plane geometry as

$$
j_{i, \text { Child }}=\frac{4}{9} \varepsilon_{0}\left(\frac{2 \bar{Q} e}{m_{i}}\right)^{1 / 2} \frac{\Phi^{3 / 2}}{d^{2}}
$$

where $\Phi$ and $d$ are the potential difference and distance between the plasma grid and the suppressor grid, respectively, $\bar{Q}$ is the mean ion charge state number, $e$ is the elementary charge, $m_{i}$ is the ion mass, and $\varepsilon_{0}$ the is permittivity of vacuum. In contrast to most other plasmas, the flow of vacuum arc ions to the plasma boundary is not governed by thermal motion but by supersonic drift acquired at cathode spots. The flow is therefore

$$
j_{i}=\bar{Q} e n_{i 0} v_{i 0}
$$


where $n_{i 0}$ and $v_{i 0}$ are the ion density and drift velocity at the plasma boundary. The average velocity of bismuth ions is $4,700 \mathrm{~m} / \mathrm{s}$, Ref. ${ }^{2}$, and $\bar{Q} \approx 1$. Perveance matching $\left(j_{i} \approx j_{i, \text { Child }}\right)$ can be accomplished by adjusting the density $n_{i 0}$ via the arc current. The vacuum arc plasma is expanding while moving away from the cathode spot. For free expansion, where anode effects and magnetic fields can be neglected, the density falls like $^{11}$

$$
n_{i 0}=\gamma I_{\text {arc }} / s_{0}^{2}
$$

where $s_{0}=13.5 \mathrm{~cm}$ is the distance from the cathode to the plasma extraction electrode, and $\gamma \approx 10^{13} \mathrm{~A}^{-1} \mathrm{~m}^{-1}$.

During the extraction voltage rise and fall times, the plasma boundary is bulging into the extraction gap, giving rise to the large beam divergence (Fig. 6(a)). In these phases, the voltage $\Phi(t)$ and associated $j_{i, \text { Child }}(t)$ are small, representing an overdense plasma regime $\left(j_{i}>j_{i, \text { Child }}\right)$, as it is observed in the head and tail phase of each beam pulse (Fig. 3). The properties in the central part of a beam pulse depend on the arc current: the supply current according to equations (3) and (2) can be smaller or greater than the Child current (1). At low arc current, the plasma is underdense $\left(j_{i}<j_{i, \text { Child }}\right)$ and the plasma boundary is more concave than ideal (Fig. 6(b)). Best beam properties are obtained under matched conditions (Fig. 6(c)).

Fluctuations of the plasma density do not only represent fluctuations in terms of ions available for extraction but they also translate into fluctuations of the shape of the plasma boundary and fluctuations of the beam envelope position. In the underdense regime, plasma density fluctuations appear amplified by ion extraction optics. This has 
been observed by the ion current variability at the beam edge, not only during the rise and fall time but throughout the beam pulse. In contrast, the central part of the beam (at small beam radii) shows only very little noise in the overdense regime, which is in agreement with previous observations ${ }^{12}$. We plan to elaborate on this effect and multi-beamlet measurements in a follow-up paper.

We gratefully acknowledge support by Joe Kwan, William Ghiorso, David Beck, William Waldron, and Lionel Prost. This work was supported by the U.S. Department of Energy under Contract No. DE-AC03-76SF00098. 
Figures and Captions

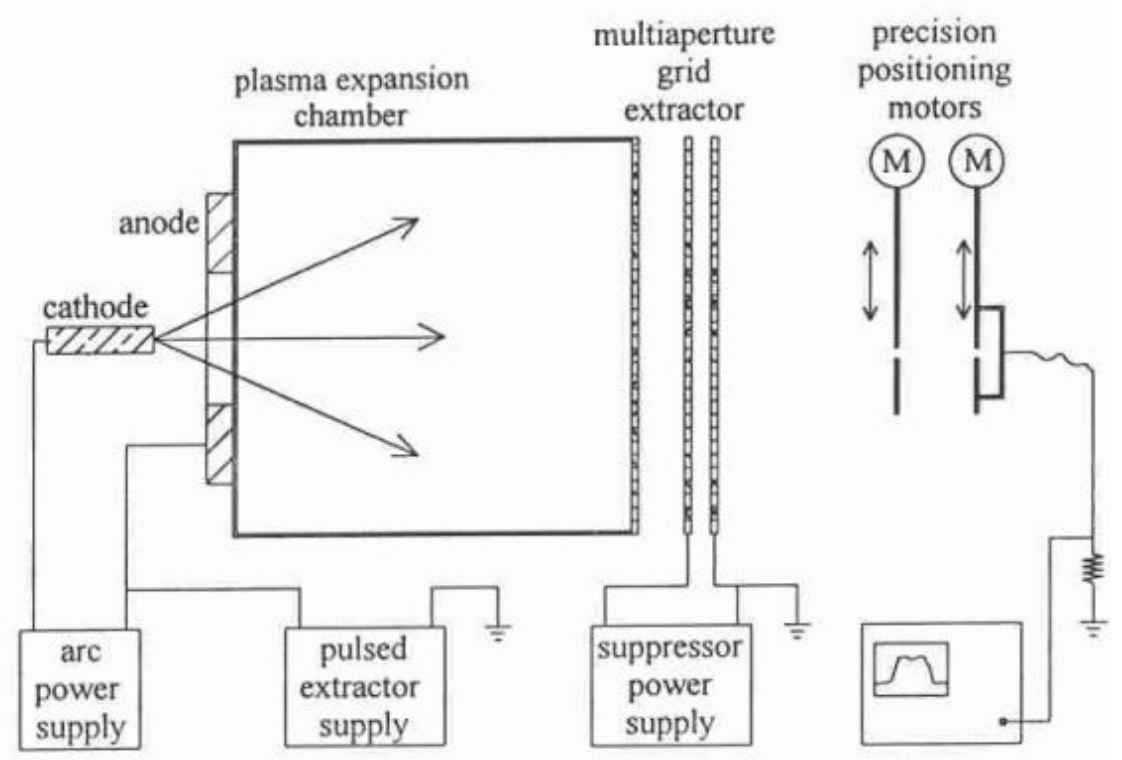

Figure 1. Vacuum arc ion source Mevva V equipped with a double slit emittance scanner. Measurements were done using the central extraction hole only.

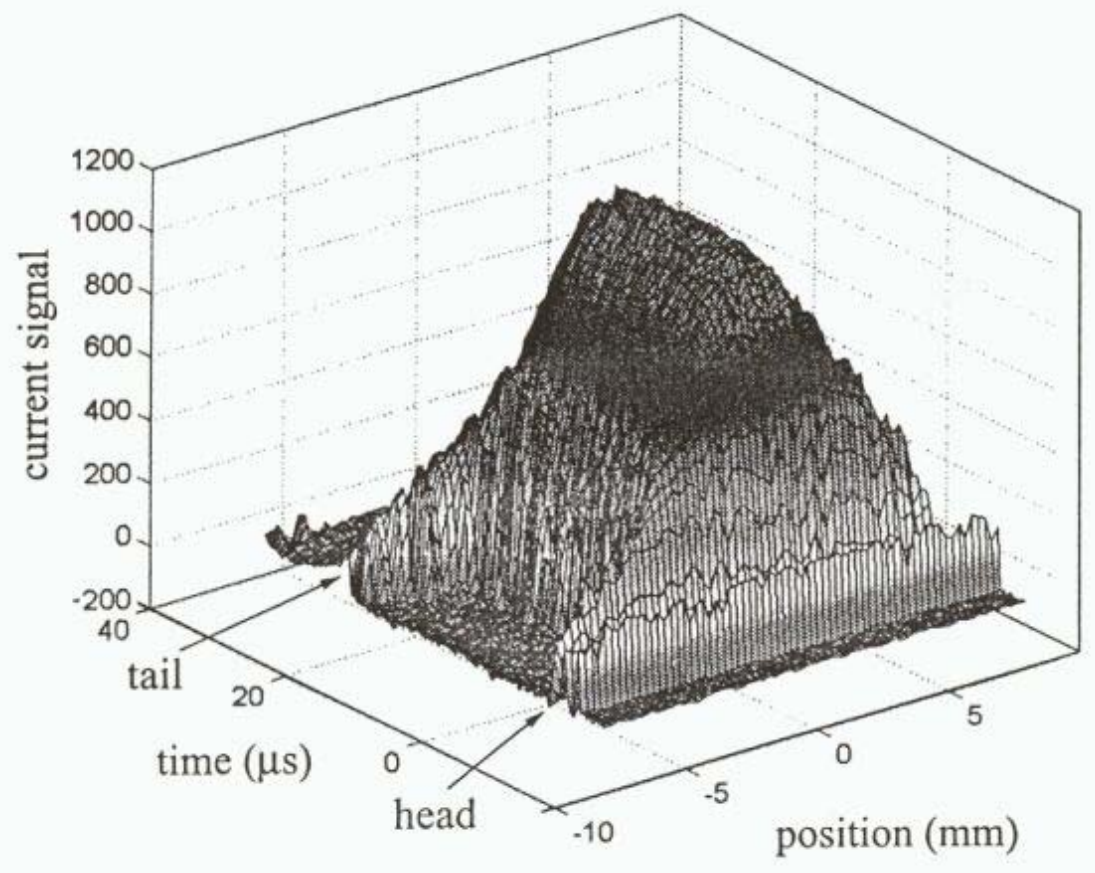

Figure 2. Time-dependent profile of a bismuth beam; arc current $160 \mathrm{~A}, 35 \mathrm{kV}$ extraction voltage. 


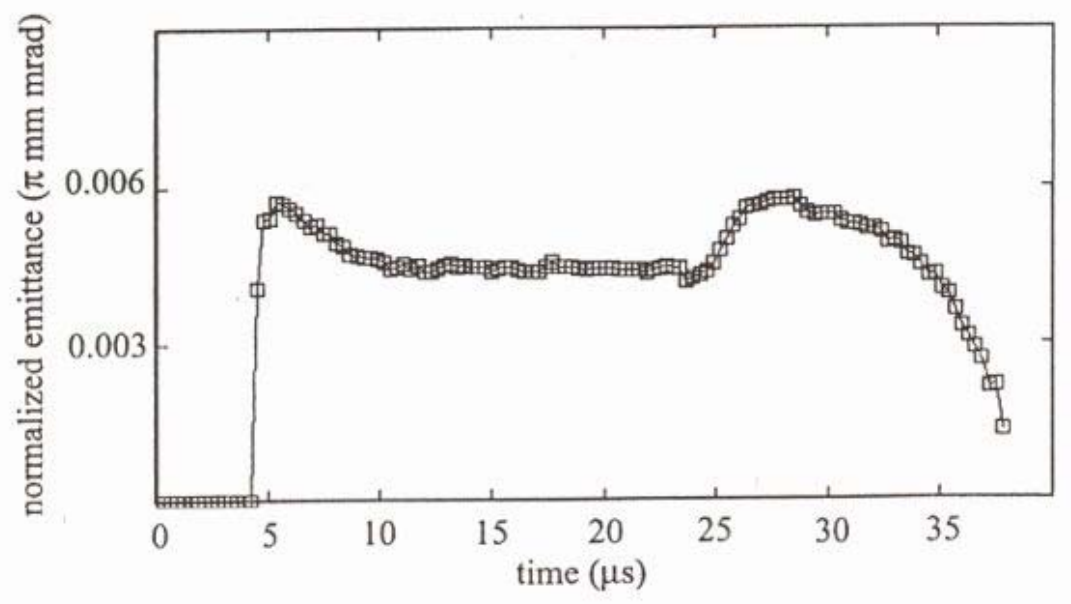

Figure 3. Time-dependent emittance of a bismuth beam with same parameters as in figure 2.

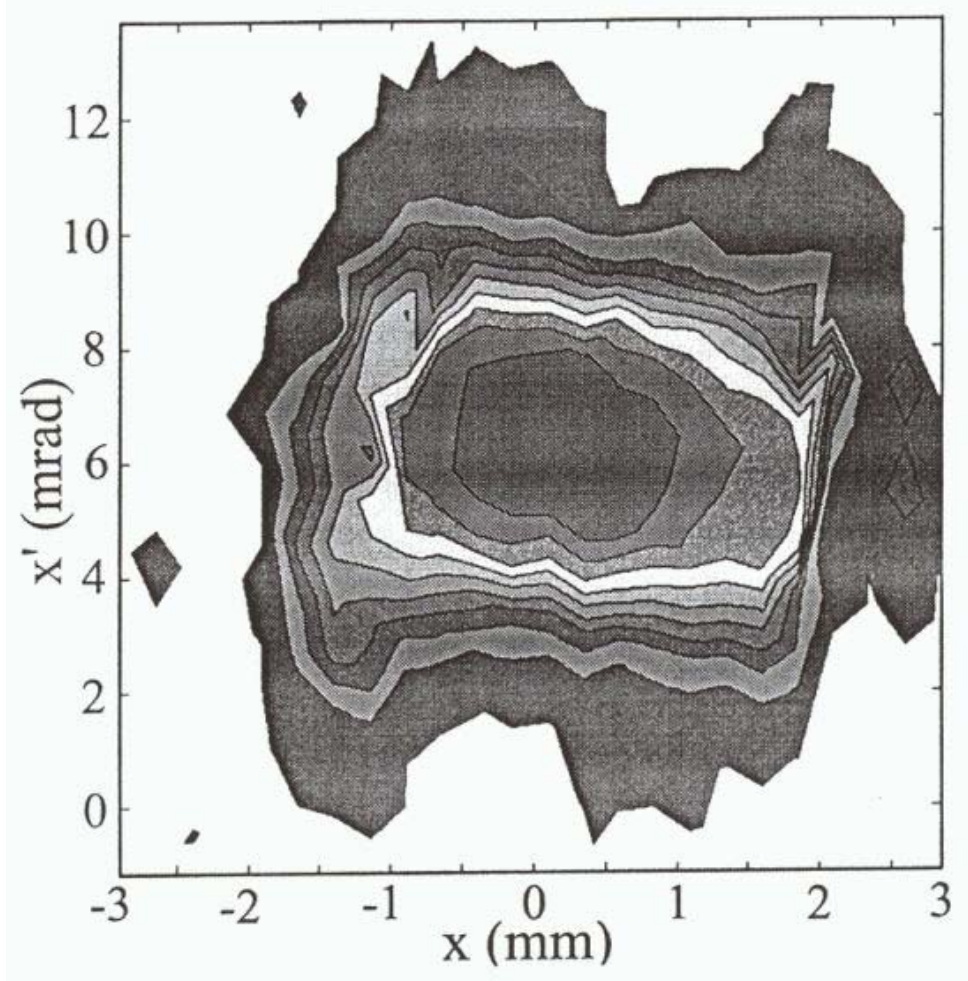

Figure 4. Emittance of a bismuth beam averaged over the time interval 10-20 $\mu$ s (i.e., excluding beam head and tail), displayed in the sheared phase space. Parameters as in figures 2 and 3 . The color scale denotes changes of $10 \%$ in signal intensity. The peak intensity is at the center of the phase space plot. 


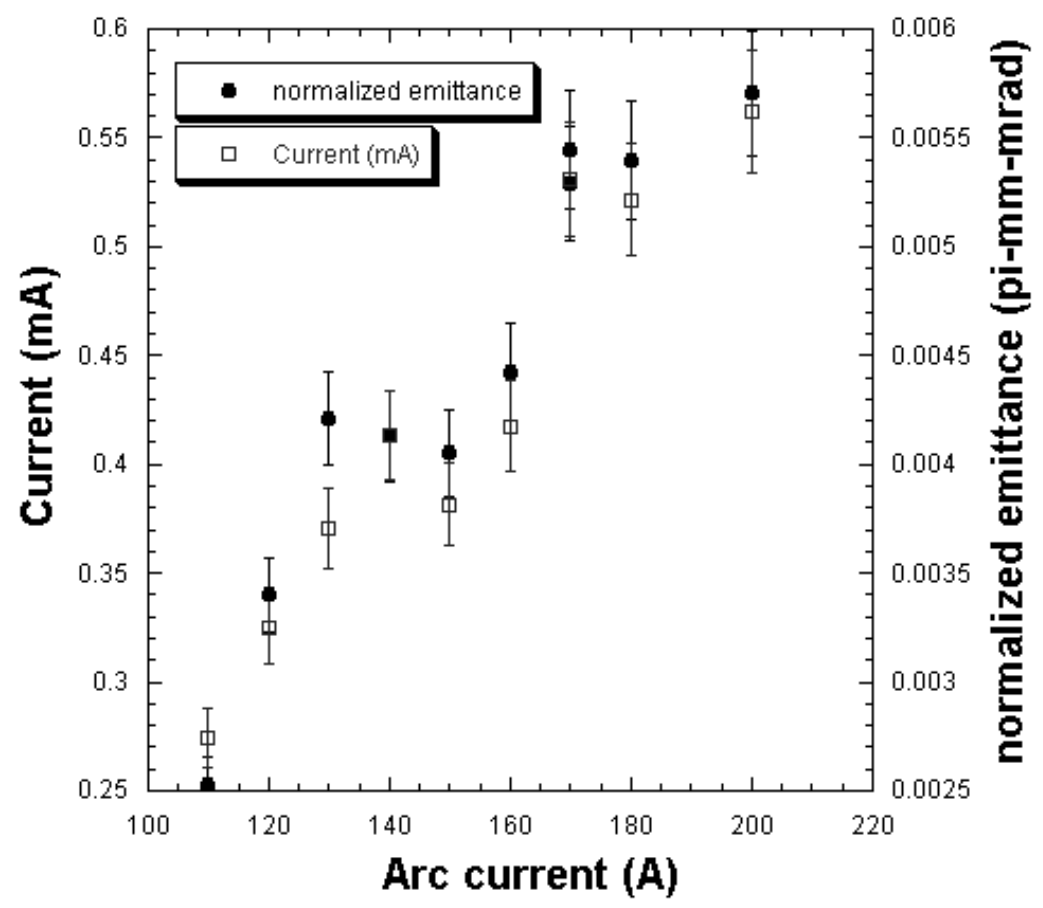

Figure 5. Emittance and ion current as a function of arc current at $35 \mathrm{kV}$ extraction voltage.

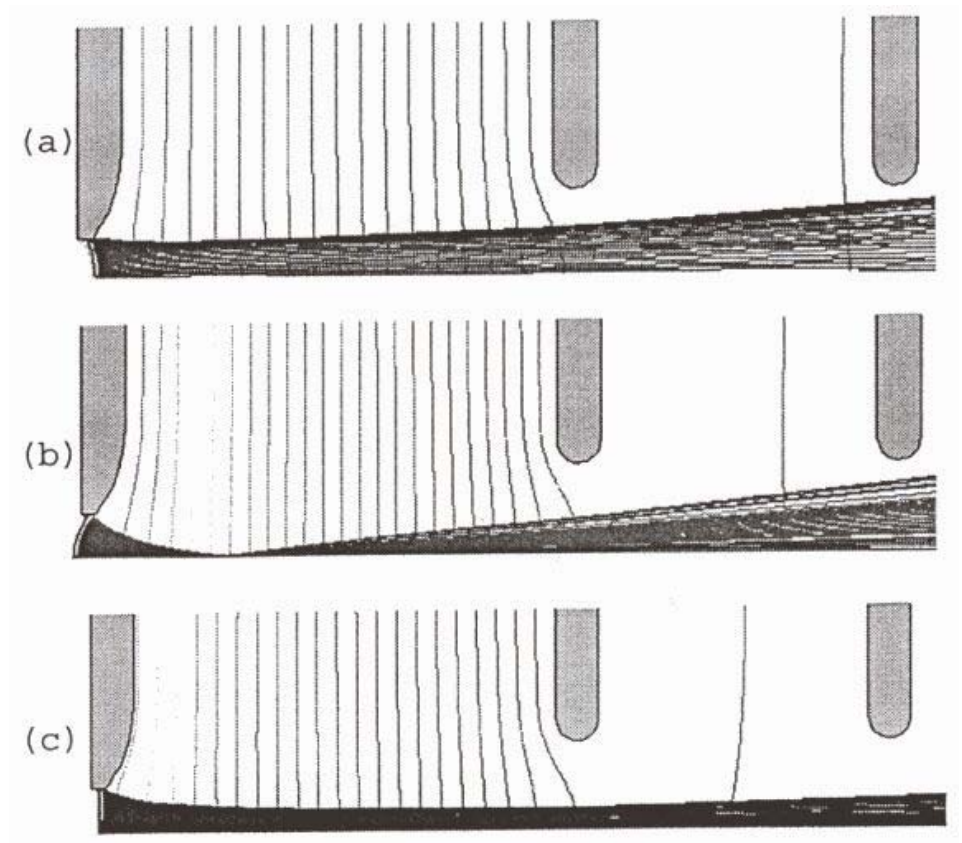

Figure 6. Ion trace simulation from a plasma boundary as a function of ion supply current and space-charge limited current, using code TRAK version 5.0. (a) oversupply condition, as typical during extraction pulse rise and fall time, leading to convex plasma boundary; (b) undersupply conditions; plasma boundary is more concave than ideal, and (c) matched condition with slightly concave plasma boundary, causing a focusing effect that compensates beam enlargement by space charge. The vertical lines represent equipotential lines. 


\section{References}

$1 \quad$ I. G. Brown, Rev. Sci. Instrum. 65, 3061 (1994).

2 A. Anders and G. Y. Yushkov, J. Appl. Phys. 91, 4824 (2002).

3 The Physics and Technology of Ion Sources, edited by I. G. Brown (Wiley, New York, 1989), pp. 23-52.

$4 \quad$ A. T. Forrester, Large Ion Beams (Wiley, New York, 1988) pp. 99-136.

5 H. L. Rutkowski, D. W. Hewett, and S. Humphries Jr., IEEE Trans. Plasma Sci. 19, $782(1991)$.

$6 \quad$ S. Humphries Jr. and H. Rutkowski, J. Appl. Phys. 67, 3223 (1990).

7 S. Humphries Jr. and C. Burkhart, Particle Accelerators 20, 211 (1987).

8 E. Oks, P. Spädtke, H. Emig, and B. H. Wolf, Rev. Sci. Instrum. 65, 3109 (1994).

9 H. Reich, P. Spädtke, and E. M. Oks, Rev. Sci. Instrum. 71, 707 (2000).

10 E. Oks, G. Yushkov, I. Litovko, A. Anders, and I. Brown, Rev. Sci. Instrum. 73, $735(2001)$.

11 B. Jüttner, J. Phys. D: Appl. Phys. 18, 2221 (1985).

12 A. Anders and R. Hollinger, Rev. Sci. Instrum. 73, 732 (2002). 